\title{
Evaluation of Various Separation Techniques for the Removal of Actinides from A Rare Earth-Containing Solution Generated from Coarse Coal Refuse
}

\author{
Deniz Talan \\ West Virginia University, dt0037@mix.wvu.edu
}

Follow this and additional works at: https://researchrepository.wvu.edu/etd

Part of the Mining Engineering Commons

\author{
Recommended Citation \\ Talan, Deniz, "Evaluation of Various Separation Techniques for the Removal of Actinides from A Rare \\ Earth-Containing Solution Generated from Coarse Coal Refuse" (2021). Graduate Theses, Dissertations, \\ and Problem Reports. 8232. \\ https://researchrepository.wvu.edu/etd/8232
}

This Dissertation is protected by copyright and/or related rights. It has been brought to you by the The Research Repository @ WVU with permission from the rights-holder(s). You are free to use this Dissertation in any way that is permitted by the copyright and related rights legislation that applies to your use. For other uses you must obtain permission from the rights-holder(s) directly, unless additional rights are indicated by a Creative Commons license in the record and/ or on the work itself. This Dissertation has been accepted for inclusion in WVU Graduate Theses, Dissertations, and Problem Reports collection by an authorized administrator of The Research Repository @ WVU.

For more information, please contact researchrepository@mail.wvu.edu. 
Evaluation of Various Separation Techniques for the Removal of Actinides from A Rare Earth-Containing Solution Generated from Coarse Coal Refuse

Deniz Talan 


\title{
EVALUATION OF VARIOUS SEPARATION TECHNIQUES FOR THE REMOVAL OF ACTINIDES FROM A RARE EARTH-CONTAINING SOLUTION GENERATED FROM COARSE COAL REFUSE
}

\section{DENIZ TALAN}

\author{
Dissertation submitted to the \\ Benjamin M. Statler College of Engineering and Mineral Resources \\ at West Virginia University
}

In partial fulfillment of the requirements for the degree of

\author{
Doctor of Philosophy \\ in \\ Mining Engineering \\ Qingqing Huang, Ph.D., Chair \\ Lian-Shin Lin, Ph.D. \\ Yi Luo, Ph.D. \\ Xueyan Song, Ph.D. \\ Ihsan Berk Tulu, Ph.D. \\ Department of Mining Engineering \\ Morgantown, West Virginia \\ 2021
}

Keywords: Rare Earth Elements, Thorium, Uranium, Coal, Separation, Fundamental Studies, Process Development 


\section{ABSTRACT \\ EVALUATION OF VARIOUS SEPARATION TECHNIQUES FOR THE REMOVAL OF ACTINIDES FROM A RARE EARTH-CONTAINING SOLUTION GENERATED FROM COARSE COAL REFUSE}

\section{DENIZ TALAN}

Rare earth elements have emerged as a vital commodity due to their significance in the production of high-tech devices as well as their utilization in defense and military systems. They are deemed critical due to potential scarcity, supply constraints, and the lack of minable concentrations. Therefore, alternative sources are needed to meet the demand and continue manufacturing rare earth-required products. Coal and coal by-products, in that regard, have presented a significant opportunity and proven to contain a considerable amount of rare earths. However, toxic elements such as thorium and uranium are frequently seen in the same mineralization as rare earths. The concentration of these hazardous trace elements is elevated due to the extraction and beneficiation processes. Unless proper separation and disposal are performed, these radionuclides accumulate on the soil's surface or integrate with aquatic systems that raise environmental and health concerns. Consequently, there exists an urgent need to remove these radionuclides to produce high purity rare earths and diversify its supply chain as well as maintain an environmentally favorable extraction process for the surroundings.

In this study, a process flowsheet was targeted to be developed for the removal of thorium and uranium from rare earth elements when coarse coal refuse was utilized as a non-traditional feedstock for rare earth production. The separation performance between these hazardous elements and rare earths was systematically evaluated. Various separation techniques, namely selective precipitation, solvent extraction, and adsorption, were applied to extract rare earth elements while minimizing the non-selective recovery of thorium and uranium into the product stream. While investigating the potential application of different separation methods, several operating parameters were tested, and the experimental test results were analyzed from a statistical and fundamental perspective to provide an in-depth understanding of each separation mechanism.

Selective precipitation test results indicate that it was effective for removing thorium, while exploratory solvent extraction tests preferentially removed uranium from rare earths. Based on the findings of the initial testing, an experimental protocol consisting of both selective precipitation and solvent extraction was developed and implemented. Around $\mathrm{pH} 4.85$, nearly all thorium was precipitated out with approximately $19 \mathrm{wt} \%$ of rare earths and $47 \mathrm{wt} \%$ uranium coprecipitation. The optimum separation performance between uranium and rare earth elements was achieved with double-stage solvent extraction under the following conditions: $50 \mathrm{v} \%$ tributyl phosphate, feed $\mathrm{pH}$ at 3.5, organic to aqueous phase ratio at 3 , and DI water as the strippant, which corresponded to an overall rare earth and uranium recovery of $80 \mathrm{wt} \%$ and 3.1 wt $\%$, respectively. The selective 
precipitation and solvent extraction test results were assessed from a fundamental point of view with respect to metal species distribution, chemical complexation reactions, and thermodynamic calculations, which all aligned well with the experimental data. The precipitation reactions were all spontaneous and exothermic for the elements studied based on calculated Gibbs energy and enthalpy values. The extraction behavior of uranium and rare earths was further studied with the distribution ratio and separation factors. Statistically, significant models were developed for uranium recovery prediction in solvent extraction, which indicates the extractant concentration, solution $\mathrm{pH}$, and organic to aqueous phase ratio all played a critical role. On the contrary, the concentration of the stripping agent was identified as an insignificant parameter over the tested concentration range.

As an alternative to selective precipitation and solvent extraction, an experimental design was generated to examine the potential application of zeolite adsorption on the removal of thorium and uranium. However, good selectivity was not observed, especially between uranium and rare earth elements. The kinetic adsorption studies suggest a similar pattern for rare earth elements and uranium, which creates a separation challenge. In the case of thorium adsorption, a fast adsorption behavior was observed. Freundlich adsorption isotherm was determined to be the best-fit model, and the adsorption mechanism of rare earths and thorium was identified as multilayer physisorption. Also, the kinetic adsorption data fit to the pseudo-second-order rate reaction for thorium and rare earth elements. The best separation performance was achieved using 2.5 grams of $12-\mu \mathrm{m}$ zeolite sample at a $\mathrm{pH}$ value of 3 with a contact time of 2 hours. Under these conditions, the adsorption recovery of rare earths, thorium, and uranium into the solid phase was found to be $20.4 \mathrm{wt} \%, 99.2 \mathrm{wt} \%$, and $89.6 \mathrm{wt} \%$, respectively.

As a result of comprehensive experimental and fundamental studies, a conceptual process flowsheet was developed using the metallurgical simulation software, METSIM. The developed flowsheet consists of five main unit operations. After dissolution with acid (1), it is intended to apply one-stage selective precipitation (2) for thorium removal followed by solvent extraction (3) for uranium separation, where two-stage extraction and two-stage stripping are performed. Later the remaining rare earth-containing aqueous stream is treated with oxalic acid to achieve rare earth oxalate precipitation (4). Finally, roasting (5) is applied to convert rare earth oxalate to rare earth oxide products. Lastly, the developed experimental protocol was validated using a real system rare earth oxalate sample produced at the pilot-scale processing facility.

KEYWORDS: Rare Earth Elements, Thorium, Uranium, Coal, Separation, Fundamental Studies, Process Development. 


\section{ACKNOWLEDGMENTS}

First of all, I would like to express my deepest gratitude to my advisor and dissertation committee chair, Dr. Qingqing Huang, for her continuous support, guidance, and motivation. This dissertation would not be possible without her valuable contributions. I will always be grateful for her patience, help, and kindness. She is an excellent researcher and advisor, and she has been a great role model to me. She gave me an opportunity of a lifetime, and I will never forget that. It was a privilege to work with her.

I would also like to extend my appreciation to my valuable committee members, Dr. Yi Luo, Dr. Lian-Shin Lin, Dr. Xueyan Song, and Dr. I. Berk Tulu, for their participation in my dissertation committee, time, and efforts. Their significant inputs enhanced the value of this dissertation, and they are greatly appreciated.

Moreover, I would like to thank Dr. Rick Honaker, Dr. Wencai Zhang, Dr. Xinbo Yang, and all the University of Kentucky Mining Engineering Department members for providing the sample used in this study and letting me benefit from their elemental analysis instrument. The efforts of West Virginia University National Resource Center for Coal and Energy Analytical Laboratory Director Gabriela Perhinschi, and Research Assistant, Robert Vincent, are also very much appreciated for conducting elemental analysis throughout my study. I am thankful to Dr. Marcela Redigolo, Dr. Qiang Wang, and Dr. Gunes Yakaboylu for their help in performing characterization tests. Further, I want to thank Dr. Aaron Noble from Virginia Polytechnic Institute and State University for his mentoring and all the external support he provided. I am grateful for his time and the knowledge he shared with me.

I would like to give special thanks to our research group members and my colleagues, Amir Eskanlou, Zhongqing Xiao, Francisco Patino, and Zeynep Cicek, who supported me constantly. They were always present when I needed their help. Also, I want to thank my friends Dr. Emel Sen Kilic, Cagri Kilic, Mustafa Can Suner, Dr. Ozcan Ozmen, and all the WVU Department of Mining Engineering students for creating great memories together in Morgantown. Moreover, I appreciate all the support and help that I received from the Faculty and Staff of the WVU Department of Mining Engineering. They all will be greatly missed.

Last but not least, I would like to express my love and appreciation to my family. I am thankful for their enormous support during my Ph.D. journey. They were on the other side of the phone whenever I needed them. Their understanding and enormous help are endless. Finally, I have to mention the tremendous support that I received from my husband, Deniz Tuncay. He lessened my burden and offered all the help he can provide. Without his help, this dissertation would not be happening. 
To My Dear Family 


\section{TABLE OF CONTENTS}

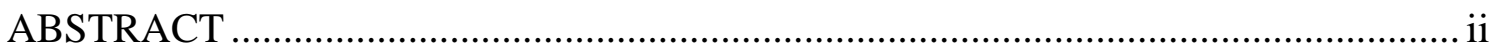

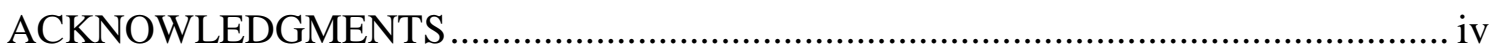

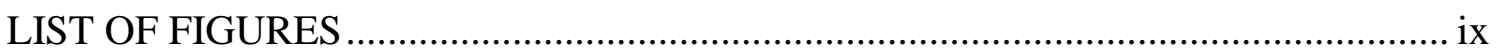

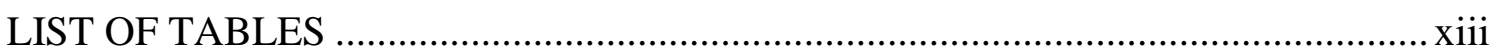

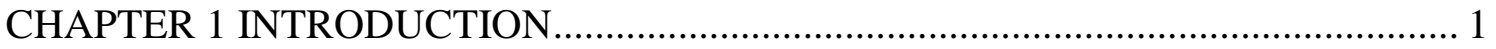

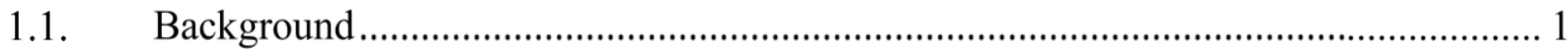

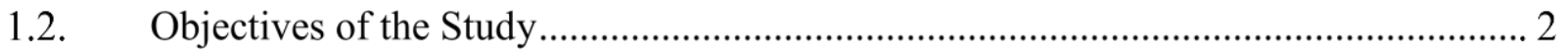

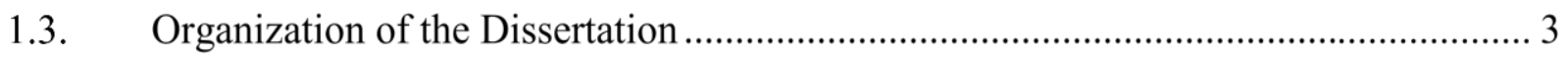

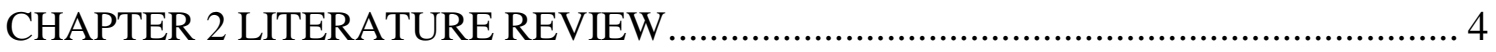

2.1. Occurrences and Applications of Rare Earth Elements............................................. 4

2.2. Recent Research Advances on Rare Earth Elements................................................. 6

2.3. Rare Earth Elements in Coal and Coal By-products................................................. 7

2.4. Environmental Impact of Rare Earth Extraction and Regulatory Stipulations............. 9

2.5. Solution Purification Techniques to Remove Thorium and Uranium from Rare Earth

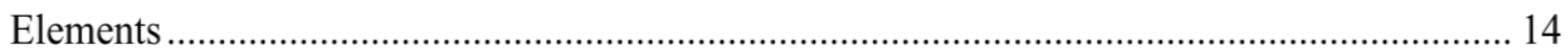

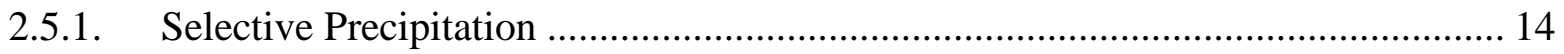

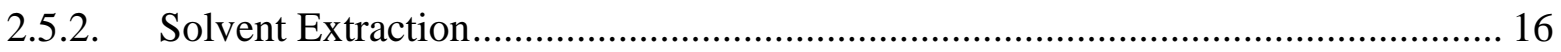

2.5.3. Solid-Phase Separation ……………………....................................................... 17

CHAPTER 3 EXPERIMENTAL AND METHODOLOGY ………………………...... 20

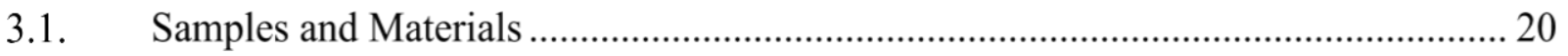

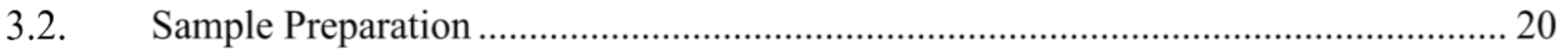

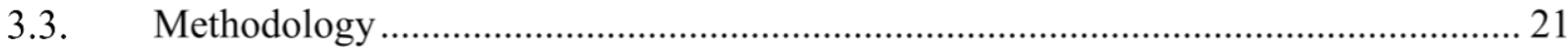

3.3.1. Preliminary Selective Precipitation Tests ............................................................. 21 


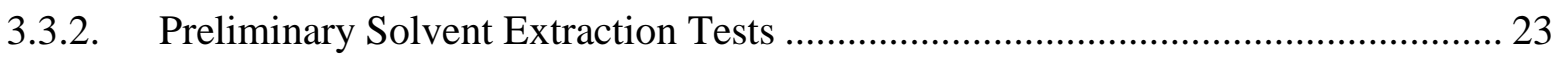

3.3.3. Modified Experimental Protocol............................................................................... 25

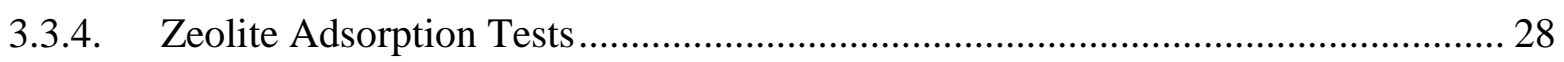

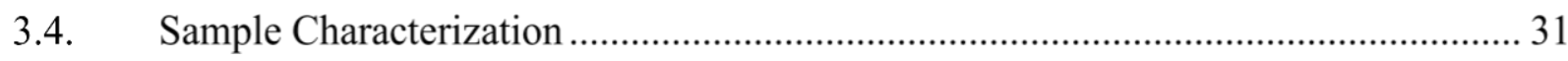

3.4.1. Characterization of Solids Generated from Selective Precipitation ........................ 31

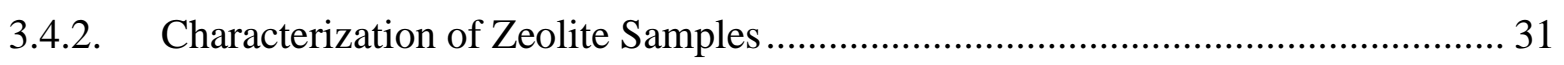

CHAPTER 4 SELECTIVE PRECIPITATION AND SOLVENT EXTRACTION

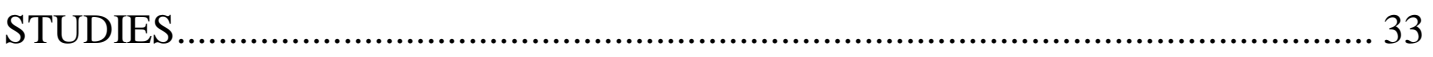

4.1. Preliminary Selective Precipitation Tests …………………………………............ 33

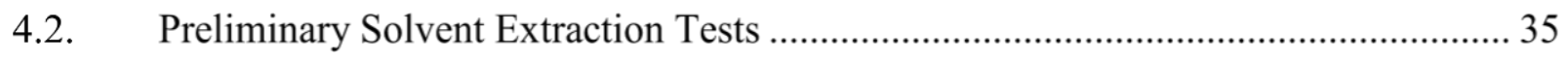

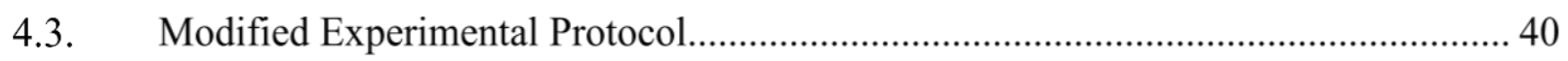

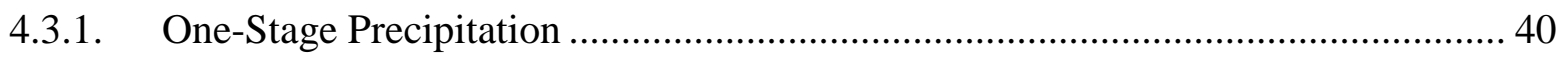

4.3.2. The First Subset of Solvent Extraction Tests.......................................................... 53

4.3.3. The Second Subset of Solvent Extraction Tests .............................................................. 61

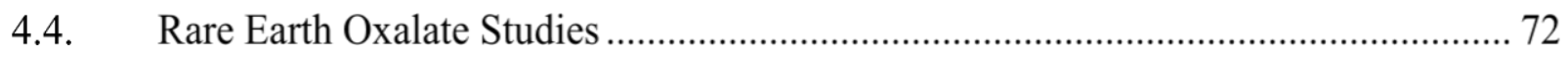

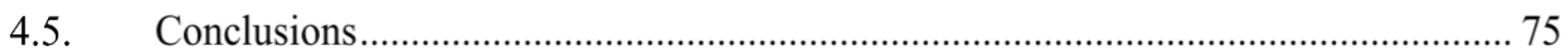

CHAPTER 5 ZEOLITE ADSORPTION STUDIES...................................................... 77

5.1. Characterization of Initial Zeolite Samples ................................................................ 77

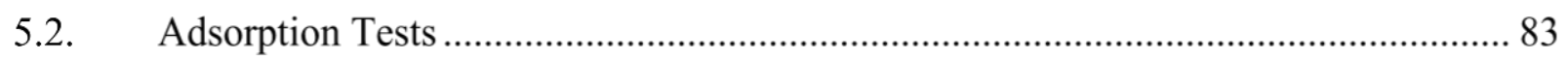

5.3. Characterization of Post Adsorption Zeolite Samples .................................................. 86

5.4. Statistical Analysis and Modeling of Adsorption Tests.............................................. 89

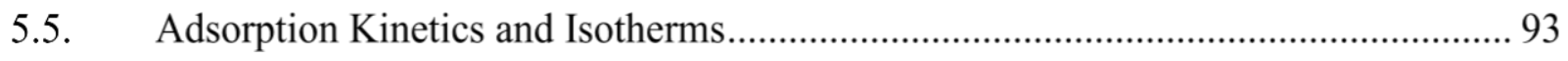

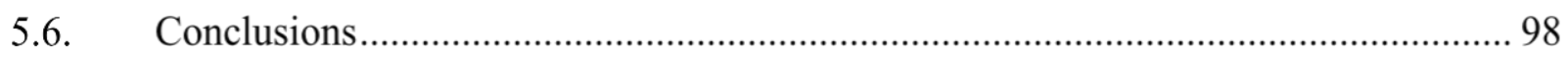

CHAPTER 6 CONCEPTUAL PROCESS FLOWSHEET DEVELOPMENT ................ 99

CHAPTER 7 CONCLUSIONS AND SUGGESTIONS FOR FUTURE STUDIES .... 102 


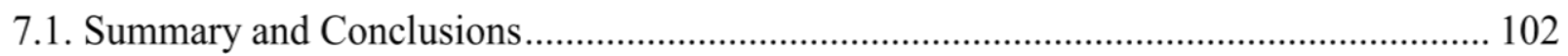

7.2. Suggestions for Future Studies............................................................................ 104

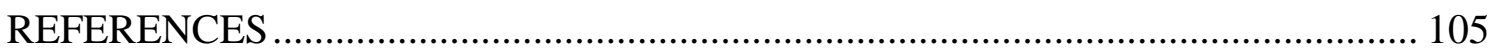

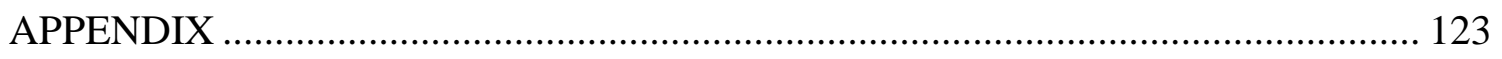

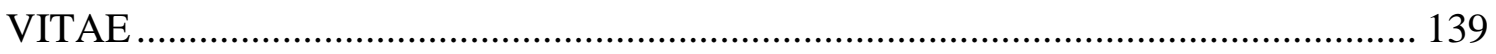




\section{LIST OF FIGURES}

Figure 1 Schematic representation of solvent extraction tests................................................. 17 Figure 2 Pictures illustrating the experimental procedures of selective precipitation tests. (A) $\mathrm{pH}$

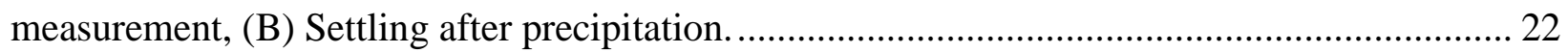
Figure 3 Experimental flowsheet followed during selective precipitation tests.......................... 22 Figure 4 Experimental flowsheet followed during the exploratory solvent extraction tests. ....... 24 Figure 5 Separation between aqueous and organic phase during solvent extraction tests............ 24 Figure 6 A modified experimental protocol developed for enhanced separation of thorium,

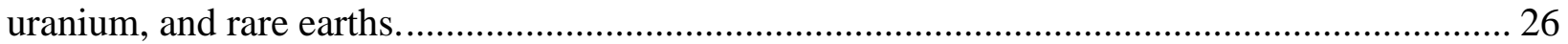

Figure 7 Experimental designs for uranium separation using TBP as the extractant: (A) DI water

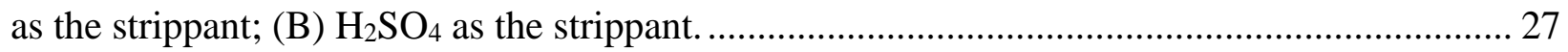

Figure 8 Structure of zeolite (after Zendelska et al., 2015). ........................................................ 29

Figure 9 Cumulative precipitation of total rare earths, thorium, and uranium ............................. 33 Figure 10 Cumulative precipitation of total, heavy, light, critical, and uncritical rare earths at

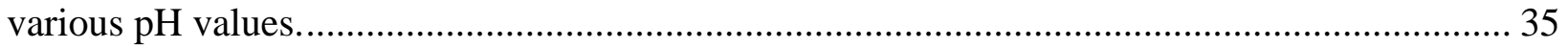

Figure 11 Recovery of total rare earths, thorium, and uranium into the REE product stream of both the one-stage and two-stage solvent extraction process. ........................................................... 37 Figure 12 Recovery of individual rare earth, thorium, and uranium into the REE product stream of both the one-stage and two-stage solvent extraction process. ...................................................... 37 Figure 13 Recovery of total, heavy, light, critical and uncritical REEs from both the one-stage and

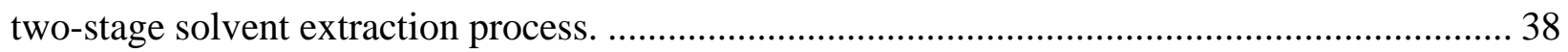
Figure 14 Average precipitation of total REEs, Th, $\mathrm{U}$, and major metals at the $\mathrm{pH}$ value of 4.85 . Error bars one standard deviation of three replicate tests.

Figure 15 The average precipitation of total, heavy, light, critical, and uncritical rare earths at the $\mathrm{pH}$ value of 4.85. Error bars represent one standard deviation of three replicate tests. ............... 42 Figure $16 \mathrm{XRD}$ pattern of the solids samples obtained from selective precipitation tests at a $\mathrm{pH}$ value of 4.85 .

Figure 17 Elemental identification of the solids samples obtained from selective precipitation at a $\mathrm{pH}$ of 4.85 using SEM-EDX analysis. 43 
Figure 18 SEM images of precipitated solids (at a $\mathrm{pH}$ of 4.85) observed under various

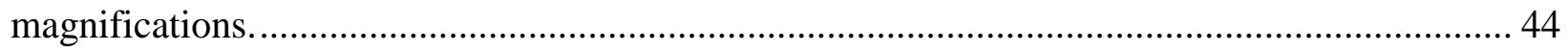

Figure 19 Species distribution diagram of $\mathrm{Th}-\mathrm{HCl}$ system. .............................................. 46

Figure 20 Species distribution diagram of $\mathrm{U}-\mathrm{HCl}$ system. .................................................. 46

Figure 21 Eh-pH diagram of Th-Cl-Na-Ca-H $\mathrm{H}_{2} \mathrm{O}$ system. ................................................ 48

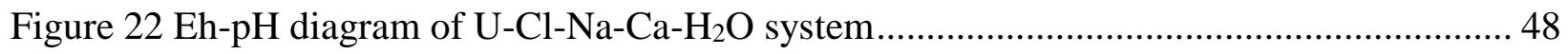

Figure 23 Species distribution diagrams of REEs-HCl system. A) Cerium, B) Neodymium, C)

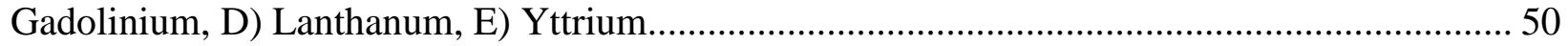

Figure 24 Species distribution diagram of $\mathrm{Fe}-\mathrm{HCl}$ system............................................... 52

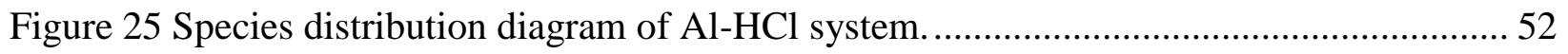

Figure 26 Experimental procedure and the operating conditions followed the second subset of solvent extraction tests 53

Figure 27 Recovery of total rare earths and uranium into the final REE product stream based solely on solvent extraction circuit. Error bars represent one standard deviation of three replicate tests.

Figure 28 Overall recovery of total rare earths and uranium with respect to the overall experimental flowsheet. Error bars represent one standard deviation of three replicate tests........................ 56 Figure 29 3D surface response graphs obtained from the statistical analysis using Design-Expert.

Figure 30 2D contour plots obtained from the statistical analysis using Design-Expert. 60

Figure 31 Experimental procedure and the operating conditions followed the second subset of solvent extraction tests

Figure 32 Recovery of total rare earths and uranium into the final rare earth product stream based solely on the solvent extraction circuit. Error bars represent one standard deviation of three replicate tests.

Figure 33 Overall recovery of total rare earths and uranium with respect to the whole experimental flowsheet. Error bars represent one standard deviation of three replicate tests. 63 Figure 34 Distribution ratio of (A) total rare earths, (B) uranium in the two extraction stages. Error bars represent one standard deviation of three replicate tests. 65 Figure 35 Separation factor between total rare earths and uranium. Error bars represent one standard deviation of three replicate tests. 
Figure 36 3D surface response graphs obtained for the statistical model predicting uranium

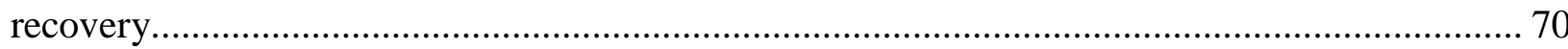

Figure $372 \mathrm{D}$ contour plots obtained for the statistical model predicting uranium recovery...... 72

Figure 38 Experimental flowsheet followed for rare earth oxalate sample............................ 73

Figure 39 Precipitation of total rare earths and thorium at various $\mathrm{pH}$ values. Error bars represent

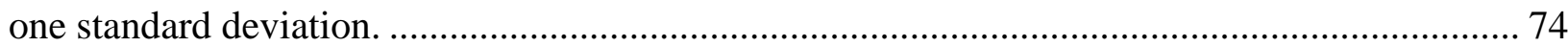

Figure 40 The SEM image of the rare earth oxide feed sample. ........................................... 74

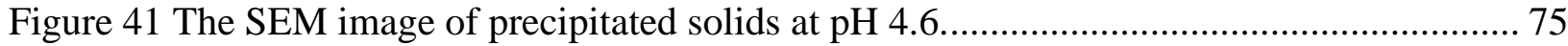

Figure 42 Particle size distribution of three zeolite samples. (A) Coarsest sample, P80=1490 $\mu \mathrm{m}$,

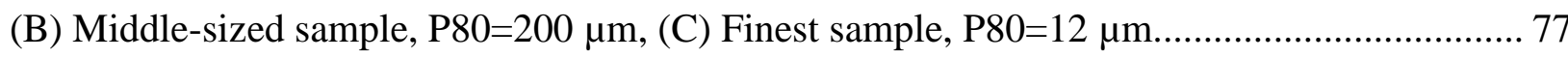

Figure 43 X-ray diffraction pattern of the zeolite samples............................................. 78

Figure 44 Elemental identification of the zeolite samples obtained from the EDX spectrums.... 79

Figure 45 SEM images of the zeolite samples under various magnification. (a) the coarsest sample,

(b) medium size sample, (c) the finest sample. 80

Figure 46 The Brunauer-Emmett-Teller (BET) Nitrogen adsorption isotherm of the three zeolite samples 82

Figure 47 Classification of (a) physisorption isotherms, (b) hysteresis (Thommes et al., 2015) . 82 Figure 48 Pore size distribution of the three zeolite samples. 83

Figure 49 Adsorption recoveries into the solid phase. A) total rare earths, B) thorium, C) uranium. Error bars represent one standard deviation of multiple replicate test.................................. 84 Figure 50 SEM image showing the fractures and cracks formed after adsorption..................... 86 Figure 51 XRD pattern of the solid samples generated with Test 11 .................................... 87 Figure 52 SEM images of the solid samples generated after adsorption. Bright areas highlighted in the red boxes indicating the deposition of heavy elements. 88 Figure 53 EDX pattern of the solid sample generated after Test 11. (A) Full EDX pattern indicating high-intensity elements. (B) Close-up view showing the detection of rare earths and thorium... 88 Figure 54 Diagnostic plots obtained based on the statistical model developed for the adsorption recovery of rare earths. 92

Figure 55 Adsorption recoveries of total rare earths, thorium, and uranium as a function of contact time. Error bars represent one standard deviation of multiple replicate tests 94 
Figure 56 Distribution coefficient values of (A) total rare earths and (B) thorium as a function of contact time. Error bars represent one standard deviation of multiple replicate tests. ............... 94 Figure 57 Pseudo-second-order reaction model for (A) total rare earths and (B) thorium.......... 95 Figure 58 Freundlich adsorption isotherms of the elements onto zeolite. (A) total rare earths (B)

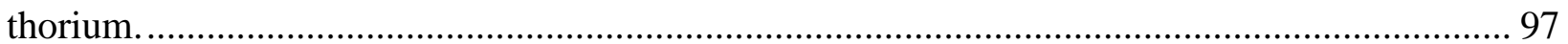
Figure 59 D-R adsorption isotherms of the elements onto zeolite. (A) total rare earths (B) thorium.

Figure 60 Conceptual flowsheet designed for the removal of thorium and uranium from rare earth elements 101

Figure 61 The PANanalytical XRD used throughout the study. 123 Figure 62 Instrument used for the SEM analysis of various solid samples. (A) Hitachi S4700 SEMEDX (B) Denton Desk V Sputter and Carbon Coater. 123

Figure 63 The CILAS particle size analyzer used throughout the study. 124

Figure 64 The Vanta handheld XRF used throughout the study. 124 Figure 65 BET nitrogen adsorption instrument used for the characterization of zeolite samples. 


\section{LIST OF TABLES}

Table 1 Classification of rare earth elements based on atomic numbers. 4

Table 2 Area of the use of rare earth elements (after Michieka, 2017 and Van Gosen et al., 2019).

Table 3 Level of radioactivity in rare earth solid waste (modified from Liu and Pan (2011)..... 11

Table 4 Radioactivity level $(\mathrm{Bq} / \mathrm{kg})$ of the nuclides in coal in various countries (modified from

World Nuclear Association (2021)) 12

Table 5 Level of radioactivity (Bq/kg) in coal solid waste (modified from Liu and Pan (2011)). 12

Table 6 Thorium and uranium concentration in the world's selected coal and coal-based materials (modified from Parzentny and Rog (2019))........................................................................ 13

Table 7 Elemental composition of synthetic feedstock solution. ......................................... 21

Table 8 Operating conditions for exploratory solvent extraction tests.................................. 23

Table 9 Test order and conditions of the solvent extraction stage following the first subset of the modified experimental protocol.

Table 10 Test order and conditions of the solvent extraction stage following the second subset of

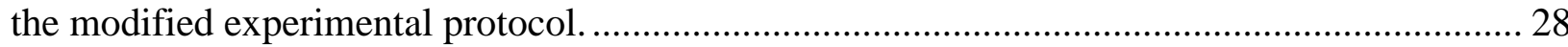

Table 11 Test order and conditions of zeolite adsorption tests........................................... 30

Table 12 Individual rare earth, thorium, and uranium concentrations (ppm) in the rare earth product stream obtained from both the one-stage and two-stage solvent extraction process.................. 38

Table 13 Individual rare earth, thorium, and uranium concentration (ppm) of the filtrate obtained

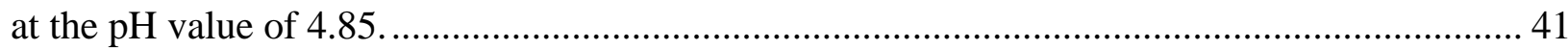

Table 14 Redox potential measurements at various $\mathrm{pH}$ values ............................................. 47 Table 15 Gibbs free energy and enthalpy and entropy values of rare earths under standard operating conditions ( 1 atm and $25^{\circ} \mathrm{C}$ ).

Table 16 Elemental composition of individual rare earth and uranium $(\mathrm{mg} / \mathrm{L})$ in the final REE product stream obtained from Test 8 . 55

Table 17 ANOVA analysis of the quadratic model for the prediction of uranium recovery........ 57

Table 18 Elemental composition of individual rare earth and uranium (ppm) in the final REE

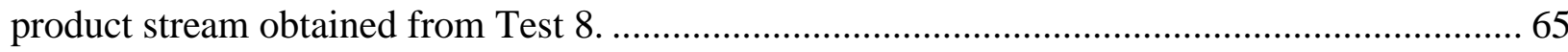

Table 19 ANOVA analysis of the quadratic model for the prediction of uranium recovery....... 67 
Table 20 Elemental composition of the rare earth oxalate sample. 72

Table 21 ANOVA analysis of the quadratic model for predicting rare earth recovery.

90

Table 22 Individual rare earth elements, thorium and uranium concentrations (ppm) in the filtrates at various $\mathrm{pH}$ values. 125

Table 23 Individual rare earth elements, thorium, and uranium precipitation (\%) at various $\mathrm{pH}$ values during the preliminary selective precipitation tests. 126 Table 24 Precipitation (\%) of heavy, light, critical, and uncritical rare earth elements at various $\mathrm{pH}$ values during the preliminary selective precipitation tests. 126 Table 25 Extraction recovery (\%) of total rare earths, thorium, and uranium during the exploratory solvent extraction tests. 126 Table 26 Extraction recovery (\%) individual rare earth elements during the exploratory solvent extraction tests. 127

Table 27 Extraction recovery (\%) of heavy, light, critical, and uncritical rare earth elements during the exploratory solvent extraction tests......

Table 28 Precipitation (\%) of total rare earth elements, thorium, uranium, and major metals during the one-stage modified experimental protocol. 127 Table 29 Individual rare earth elements and uranium extraction recovery (\%) into the final rare earth product stream based on solely solvent extraction circuit during the first subset of solvent extraction tests. 128

Table 30 Individual rare earth elements and uranium concentrations $(\mathrm{mg} / \mathrm{L})$ in the final rare earth product stream based on solely solvent extraction circuit during the first subset of solvent extraction tests.

Table 31 Overall extraction recovery $(\%)$ of total rare earth elements and uranium during the first subset of solvent extraction tests. 130 Table 32 Individual rare earths and uranium extraction recovery (\%) into the final rare earth product stream based solely on the solvent extraction circuit during the second subset of solvent extraction tests. 131 Table 33 Individual rare earth elements and uranium concentrations $(\mathrm{mg} / \mathrm{L})$ in the final rare earth product stream based solely on solvent extraction unit during the second subset of solvent extraction tests. 133 
Table 34 Overall extraction recovery (\%) of total rare earth elements and uranium during the first

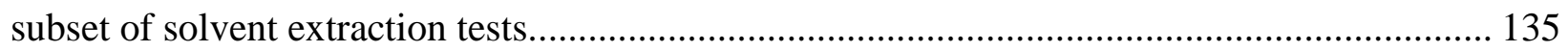

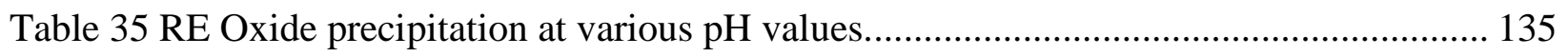
Table 36 Adsorption recovery of total rare earths, thorium, and uranium during zeolite adsorption

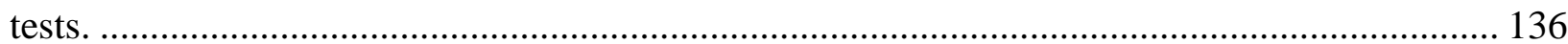
Table 37 Individual rare earth elements, thorium and uranium concentrations $(\mathrm{mg} / \mathrm{L})$ in the aqueous filtrate after zeolite adsorption tests......................................................................... 137 Table 38 The results of the kinetic adsorption tests............................................................... 138 


\section{CHAPTER 1}

\section{INTRODUCTION}

\subsection{Background}

Rare earth elements (REEs) are essential raw materials for numerous applications such as hightech industries, military, and defense systems (Binnemans et al., 2013; Wang et al., 2017). In recent years, there has been a growing interest in exploring new and alternative sources to meet the industrial demand, and the deficit arose due to the tight export quotas favored by the world's leading rare earth producer and supplier, China (Alex et al., 1998).

Recently, in addition to the primary rare earth sources (i.e. bastnaesite, monazite, and xenotime), coal and coal byproducts have been distinguished as potential feedstock materials for rare earth elements (Seredin, 1996; McLellan et al., 2014; Hower et al., 2016; Zhang and Honaker, 2018; Huang et al., 2018). According to the characterization studies conducted in various geological locations, the average concentration of rare earths in coal has been identified as $69 \mathrm{ppm}$ on a whole sample basis (Ketris and Yudovich, 2009). Also, it was stated in a study that the total amount of rare earths in coal is nearly 50 million metric tons, which accounts for $50 \%$ of REEs in conventional rare earths deposits (Yang, 2019). Considering the vast amount of coal reserves and the volumes of byproducts generated after coal mining and processing, the utilization of which has the possibility to overcome the challenges the rare earth industry facing and contribute to the U.S. economy. Currently, with no domestic supply chain, the U.S. is vulnerable to interruptions in the international market. Therefore, developing a rare earth supply chain is of great importance for the U.S.

Occurrences of rare earth elements and their extraction techniques have been well summarized by many research studies (Kuzmin et al., 2012; Seredin and Dai, 2012; Zhang et al., 2015; Van Gosen et al., 2017; Arbuzov et al., 2018). Nonetheless, rare earth extraction processes cause significant environmental issues by producing mildly radioactive tailing streams due to the association of naturally occurring radioactive materials (NORM), such as thorium (Th) and uranium (U). The radioactive materials can be enriched along with rare earths since they also dissolve during the leaching of host minerals (Al-Areqi et al., 2015), and these dissolved radioactive ions, pollute the groundwater and surface water (Akkaya, 2013). Although, there are studies conducted to remove the hazardous elements selectively, the focus of which are generally on the conventional sources of rare earths (Jun et al., 1998; Rabie et al., 2007; Anufrieva et al., 2014; Panda et al., 2014; Batchu et al., 2015; Borai et al., 2016; Dittrich et al., 2016; Alemrajabi et al., 2017; Kuang et al., 2017; Pillai, 2017). However, when coal and its byproducts are identified as potential sources for rare earth elements, it is equally crucial to investigate promising separation techniques to efficiently separate hazardous and radioactive elements from rare earths in a coal-based matrix (Valkov et al., 2014; Zhang et al., 2016, Wang, et al., 2017). 


\subsection{Objectives of the Study}

This study was supported by the Department of Energy's National Energy Technology Laboratory under the Award Number DE-FE0027035. The ultimate goal of the overall project was to design, construct and test a mobile pilot-scale plant that is capable of processing $1 / 4$-ton per hour of coal or coal by-products to produce concentrates assaying more than $2 \%$ rare earth elements on a dry, whole mass basis in an environmentally and economically benign manner.

Per the project goal, this Ph.D. dissertation specifically focused on environmental control, which aims to investigate the potential application of various separation techniques to remove radioactive and hazardous elements; thorium and uranium. At the end of detailed experimental and fundamental studies, the development of an effective separation process to remove thorium and uranium with optimum operating parameters was targeted. Besides, it was intended to contribute to the literature by providing insight into the theoretical and experimental understanding of the separation processes.

The research tasks to achieve the dissertation objectives included the followings:

1. Investigation of selective precipitation, solvent extraction, and zeolite adsorption to explore the potential of removing thorium and uranium from a rare earth-containing solution,

2. For each separation technique, exploration of the effect of various influential operating parameters on the selective removal of thorium and uranium,

3. Utilization of experimental design tools to identify the test conditions as well as optimize the processes from the operational standpoint,

4. Assessing the process separation efficiency of each separation technique using the response surface methodology (RSM), and utilization of the statistical tool to test the significance and adequacy of the models developed during the study,

5. Conducting detailed characterization studies using various analytical techniques such as $\mathrm{X}$-ray diffraction (XRD), X-ray fluorescence (XRF), scanning electron microscope-energy dispersive X-ray spectroscopy (SEM-EDX), Brunauer-Emmett-Teller (BET) nitrogen adsorption, and the particle size analyzer (PSA) to identify the chemical and mineralogical compositions, surface morphology, particle size distributions as well as pore size distribution and surface area of a variety of solid samples,

6. Performing fundamental studies, which include equilibrium, kinetic and thermodynamic studies, to obtain an in-depth understanding of each separation mechanism. The fundamental studies cover the species distribution for selective precipitation to illustrate the relative solubility, stability regions of different species, and predominant species at a given $\mathrm{pH}$ value. Studies for extraction mechanisms of certain elements cover distribution ratio and separation factor. Studies for the adsorption technique contain the development of Langmuir, Freundlich, and Dublinin-Raduchlevitch isotherms based on the experimental data to have an in-depth understanding of the adsorption mechanism. Thermodynamic studies include determining the Gibbs standard free energy change, standard enthalpy, and entropy changes. 


\subsection{Organization of the Dissertation}

The dissertation was divided into seven chapters. The first chapter gives a brief introduction to the background and objectives of the study. The second chapter covers a thorough literature review on rare earth elements and their applications in various areas, followed by the research and development studies performed on substitution and recycling of rare earths sources. The occurrences of rare earth elements in coal and coal byproducts and the environmental issues that arise during rare earth extraction were also provided in detail. Besides, regulatory stipulations established by various agencies and countries were summarized in addition to the techniques used for the separation of radioactive and hazardous elements from rare earths.

In chapter three, sample preparation, apparatus, and experimental methodologies were introduced. The results and discussions of the experimental findings were presented in chapters four and five. While chapter four includes the studies on selective precipitation and solvent extraction, chapter five focuses on zeolite adsorption studies. Following the experimental results, in chapter six, the conceptual process development was described. Lastly, in chapter seven, the conclusions obtained in this dissertation were compiled and listed in addition to the suggestions for future studies. 


\section{CHAPTER 2}

\section{LITERATURE REVIEW}

\subsection{Occurrences and Applications of Rare Earth Elements}

Rare earth elements or lanthanides refer to 15 elements that occur together in the periodic table and have atomic numbers from 57 to 71 . Two other elements, scandium and yttrium with an atomic number of 27 and 39, respectively, are also considered as rare earth elements since they share similar chemical and physical properties (Rim et al., 2013; Jorjani and Shahbazi, 2016; Anderson et al., 2017; Zhou et al., 2018). Contrary to their names, the abundance of rare earths in the earth's crust is much higher than many commodities (Jordens, 2016; Van Gosen et al., 2017). Even the least abundant rare earths, thulium and lutetium, have an average crystal abundance of approximately 200 times of gold. As opposed to thulium and lutetium, cerium, yttrium, lanthanum, and neodymium are the most abundant rare earths. However, concentrated rare earth deposits are limited, accounting for ineffective and uneconomical extraction (Fouquet and Martel-Jantin, 2014; McLallan et al., 2014).

On the basis of the atomic weight, rare earths are divided into two groups. The elements from lanthanum through gadolinium comprise the light rare earth elements (LREEs), whereas the rest forms heavy rare earths (HREEs), which are more in demand than lighter ones because of their higher strategic value (Van Gosen et al., 2019). Although yttrium has an atomic number of 39, it is included in the heavy rare earth group due to its similar characteristics as other heavy rare earths. A detailed list of heavy and light rare earths is provided in Table 1.

Table 1 Classification of rare earth elements based on atomic numbers.

\begin{tabular}{|c|c|c|c|c|c|}
\hline \multicolumn{3}{|c|}{ Light Rare Earth Elements (LREEs) } & \multicolumn{3}{|c|}{ Heavy Rare Earth Elements (HREEs) } \\
\hline Element Ator & No. & Atomic Wt. (g/mol) & Elemen $\mathrm{A}$ & Atomic No. & Atomic Wt. $(\mathrm{g} / \mathrm{mol})$ \\
\hline $\begin{array}{l}\text { Lanthanum } \\
\text { (La) }\end{array}$ & 57 & 138.91 & $\begin{array}{l}\text { Yttrium } \\
(\mathrm{Y})\end{array}$ & 39 & 88.91 \\
\hline $\begin{array}{l}\text { Cerium } \\
(\mathrm{Ce})\end{array}$ & 58 & 140.12 & $\begin{array}{l}\text { Terbium } \\
\text { (Tb) }\end{array}$ & 65 & 158.92 \\
\hline $\begin{array}{l}\text { Praseodymium } \\
\text { (Pr) }\end{array}$ & 59 & 140.91 & $\begin{array}{l}\text { Dysprosium } \\
\text { (Dy) }\end{array}$ & 66 & 162.50 \\
\hline $\begin{array}{l}\text { Neodymium } \\
(\mathrm{Nd})\end{array}$ & 60 & 144.24 & $\begin{array}{l}\text { Holmium } \\
\text { (Ho) }\end{array}$ & 67 & 164.93 \\
\hline $\begin{array}{l}\text { Samarium } \\
(\mathrm{Sm})\end{array}$ & 62 & 150.36 & $\begin{array}{l}\text { Erbium } \\
(\mathrm{Er})\end{array}$ & 68 & 167.26 \\
\hline $\begin{array}{l}\text { Europium } \\
(\mathrm{Eu})\end{array}$ & 63 & 151.96 & $\begin{array}{l}\text { Thulium } \\
\text { (Tm) }\end{array}$ & 69 & 168.93 \\
\hline \multirow[t]{2}{*}{$\begin{array}{l}\text { Gadolinium } \\
\text { (Gd) }\end{array}$} & 64 & 157.25 & $\begin{array}{l}\text { Ytterbium } \\
(\mathrm{Yb})\end{array}$ & 70 & 173.04 \\
\hline & & & $\begin{array}{l}\text { Lutetium } \\
(\mathrm{Lu})\end{array}$ & 71 & 174.97 \\
\hline
\end{tabular}


The formation of rare earths is diverse and complex. They do not occur as native elemental metals; instead, they are seen in various host minerals, such as halides, carbonates, oxides, phosphates, and silicates (Rim et al., 2013; Suli et al., 2017). Although there are more than 200 rare earthbearing minerals being identified in nature, bastnaesite $(\mathrm{REECO} 3 \mathrm{~F})$, monazite ((Th,REE) $\left.\mathrm{PO}_{4}\right)$, xenotime $\left(\mathrm{REEPO}_{4}\right)$, and ion-adsorption clays are considered as the primary sources due to their REE content and economic feasibility, and account for more than $95 \%$ of total rare earth production (Amaral and Morais, 2010; Hidayah and Abidin, 2017). The world's two largest rare earth deposits, Mountain Pass (USA) and Bayan Obo (China), have bastnaesite as their rare earth host mineral, which is particularly rich in light rare earths. Monazite is generally seen in sediments and recovered especially in coastal deposits. The deposits in Australia, Brazil, China, India, Malaysia, and South Africa constitute the largest monazite deposits. Currently, it is extracted on the east coast of Brazil and India (Van Gosen et al., 2017). Oppositely, xenotime contains a high concentration of heavy rare earths and yttrium (Alex et al., 1998). Like xenotime, ion adsorption clays also contain a considerable amount of heavy rare earths (typically $300 \mathrm{ppm}$ ), which are different than the mineral-type (i.e., bastnaesite, monazite, and xenotime) deposits, and discovered in granite weathering crust in Southern China in the late 60s (Wang et al., 2018). Apatite, cheralite, eudialyte, loparite, and phosphorites make up the remaining secondary sources (Dutta et al., 2016).

Because of their unique magnetic, optical, and electrical properties, rare earth elements have diverse industrial applications (Navarro and Zhao, 2014; Wang et al., 2017). The military uses rare earths for the production of night-vision goggles, communication, and GPS equipment. Likewise, they are used in developing defense systems, such as jet engines, missile guidance systems, and satellites (Van Gosen et al., 2019). They are heavily used in the production of computers, cell phones, electric vehicle batteries, and many other electronic devices. In addition to their critical role in modern technology, energy, defense, and military industries, they are highly used in glass, petroleum, automotive, medical, and ceramic industries (Banda et al., 2012; Yoon et al., 2016). Lanthanum-based catalysts and cerium-based catalysts are commonly used in petroleum and automotive industries, whereas cerium oxide, lanthanum, and lutetium are widely used in the glass industry to provide polishing, color, and unique optical properties (Van Gosen et al., 2017). Producing permanent magnets from rare earth alloys is an expanding area and neodymium is used to produce neodymium-iron-boron magnet, which is the strongest type of magnet (Uda et al., 2000). Permanent magnets are also used in electric motor production for hybrid cars and wind turbines. Moreover, gadolinium phosphorous is used for X-ray imaging and medical applications such as MRI, and yttrium, lanthanum, praseodymium is used as pigments for ceramics (Akdogan and Ghosh, 2014). In Table 2, example applications are given for each of the rare earth elements. 
Table 2 Area of the use of rare earth elements (after Michieka, 2017 and Van Gosen et al., 2019).

\begin{tabular}{c|c}
\hline Element & Application \\
\hline Scandium & Super-alloys, ultra-light aerospace components, X-ray tubes \\
Cerium & Catalytic converters, steel production, colored glass \\
Praseodymium & Super-strong magnets, lasers \\
Neodymium & Guidance and communication systems \\
Samarium & Permanent magnets, precision-guided weapons \\
Europium & Fluorescents and phosphorus in lamps and monitors \\
Gadolinium & Magnetic resonance imaging contrast agent, nuclear reactor shielding \\
Terbium & Fuel cells, sonar systems \\
Dysprosium & Batteries for hybrid gas-electric vehicles, magnets for wind turbines \\
Holmium & Catalysts, magnets, lasers \\
Erbium & Amplifiers in fiber-optic data transmission \\
Thulium & Portable X-ray machines, microwaves, metal halide lamps \\
Ytterbium & Infrared lasers, chemical reducing agents, rechargeable batteries \\
Lutetium & Superconductors, LED lamps \\
Yttrium & Cancer treatment drugs, alloys, high-intensity lamps \\
\hline
\end{tabular}

\subsection{Recent Research Advances on Rare Earth Elements}

Although the average amount of rare earths needed per unit product has decreased, due to the increase in global population increase and the expanded area of use, the demand for rare earth elements is rapidly increasing. It became a significant issue in 2011 when the market values of rare earths increased by almost 200\% (Paul and Campbell, 2011; Paulick and Machacek, 2017). Besides, it raises a serious concern among many countries since most rare earth deposits are located in China. Considering the global reserves, China holds approximately $48 \%$ while the U.S., India, and Australia hold $12 \%, 3 \%$, and $1 \%$, respectively. Within that context, research and development studies have gained tremendous significance.

The global reserves for rare earth elements were estimated at 130 million metric tons (Gamboghi, 2015). However, the industrial demand is expected to increase even more, and the value of the rare earth market is projected to be more than $\$ 20$ billion by 2024, which was $\$ 9$ billion in 2019 (Song et al., 2017; Yang et al., 2020). For some specific elements, such as dysprosium, the demand is projected to increase by $2600 \%$ over the next decade (Honaker et al., 2018). Due to their importance in many industries and shortage in supply, the U.S. Department of Energy recognized neodymium (Nd), terbium ( $\mathrm{Tb}$ ), yttrium (Y), dysprosium (Dy), and europium (Eu) as critical rare earths (CREEs) (Chu, 2011; Quinn et al., 2015). Besides, depleting resources, environmental concerns of rare earth mining, and the export limitations set by the world's biggest rare earth producer and supplier, China, have urged countries to develop various research projects to recycle, substitute, and explore alternative sources in compliance with the environmental regulations (U.S. Department of Energy, 2011; McLellan et al., 2014; Das et al., 2018). 
Within this context, new operations were initiated in India, Brazil, and Vietnam to produce rare earths as a byproduct of rutile and zircon production (Paulick and Machacek, 2017). Meanwhile, Russia and Malaysia started to recover rare earths from magnesia and cassiterite plants, respectively (Van Gosen et al., 2017). New deposits have been identified in Canada, the United Kingdom, Estonia, and Greenland (Suli et al., 2017). A probable mineral reserve that accounts for $0.59 \%$ of total rare earth oxide has been identified in Sweden (Schreiber et al., 2016) while another mineral resource was estimated in Alaska with $0.56 \%$ total rare earths.

Furthermore, two previously operated mines, Mountain Pass in the U.S. and Mount Weld in Australia, were re-opened to increase their respective domestic rare earth production. The Australian company, Laynas Corporation, may break the dominancy of China by becoming the world's largest rare earth processing plant if it fully operates. Besides, both the U.S. Department of Energy and the U.S. Critical Materials Institute support multiple high-value projects to develop substitutes for rare earths and other critical elements (Paul and Campbell, 2011). Nickel-laterites of Cuba and the Dominican Republic have also been identified as potential rare earth sources for the future (Dutta et al., 2016). Alternatively, bauxite residue (red mud) has a considerable rare earth concentration, particularly high Scandium content. While the rare earth concentration in Greek bauxite is $506 \mathrm{ppm}$, it can be as high as $2500 \mathrm{ppm}$ for the Jamaican bauxite samples (Binnemans et al., 2015).

The research initiatives on recycling electronic wastes to be used as alternative rare earth sources has tremendous potential. According to 2016 data, 44.7 million tons of electronic waste were generated in the world, which is even projected to be greater in the future (Jyothi et al., 2020). The European Union is sponsoring a variety of ongoing research projects that mostly focus on recycling rare earths from permanent magnets, disused metal alloys, and fluid catalytic cracking catalysts. For instance, a Belgian-based company has developed a program to recycle rare earths from nickelmetal-hydride (NiMH) batteries (Van Gosen et al., 2017). Similarly, Germany is working on recycling rare earths from used light metal alloys and catalysts to contribute to the European supply chain. Solvay, an international chemical company, is working on recycling light bulbs to recover cerium and lanthanum in France. In Asia, Honda Motor Co. Ltd. and Hitachi Ltd., are working to recycle rare earths from batteries, hard disk drives, air conditioners, and other types of compressors (Van Gosen et al., 2017). In terms of recycling, as reported in Omodara and his coworkers' study (2019), a novel process has been developed to recover light rare earth elements with a purity greater than $99 \%$ from shredded hard disk drives by the researchers at Critical Minerals Institute and Ames Laboratory. Alternatively, Hua et al. (2014) and Jakobsson et al. (2016) proposed the extraction of rare earths by molten chlorides and direct melting of $\mathrm{NdFeB}$ magnets, separately.

\subsection{Rare Earth Elements in Coal and Coal By-products}

Lately, as one of the outcomes of the research and development efforts, there have been studies validating the technical feasibility of coal and coal byproducts as alternative sources for REEs (Akdogan and Ghosh, 2014; Hower et al., 2016; Kolker et al., 2017; Das et al., 2018; Huang et al., 2018; Lanzerstorfer, 2018; Lin et al., 2018; Valentim et al., 2019). 
Rare earth concentrations in coal and coal byproducts vary between 270 and 1,480 ppm (Blissett et al., 2014; Das et al., 2018). According to several studies from different geological locations, average REE content in coal has been estimated to be $69 \mathrm{ppm}$ on a whole mass basis (Eskanazy, 1987; Ketris and Yudovich, 2009; Karayigit et al., 2000; Hu et al., 2006; Wagner and Matiane, 2018; Huang et al., 2019) while the REE concentration in the U.S. coals was indicated as $62.1 \mathrm{ppm}$ (Seredin and Dai, 2012). The average REE content in coal fly ashes has been determined as 445 ppm (Pan et al., 2019). On the other hand, a study conducted with 86 coal ash samples from the U.S. revealed that the total rare earth concentration in coal ash fluctuated between 98 ppm to 525 ppm, in which samples from West Virginia contain the highest concentration (Das et al., 2018). This finding is also supported by Lin et al. (2018) and Ekmann (2012), both of which indicate that the Appalachian region has the highest probability of being a source for rare earth recovery. In another study, the Pennslyvania Department of Environmental Protection stated that massive coal refuse piles are overlaying 10,000 acres and Yang et al. (2020) estimated the rare earth concentration in those piles to be between 200-500 ppm. Akdogan and Ghosh (2014) studied the rare earth content in several Alaskan coal and ash samples. They stated that the inorganic component of the examined Alaskan coals has a higher rare earth concentration than the organic component. As another byproduct of coal, bottom ash reportedly contains 110 ppm with a dominance of La, Ce, and $\mathrm{Nd}$ (Allegrini et al., 2014).

According to the study conducted by Honaker et al. (2016), the REE distribution in coal was found to be associated with the incombustible material in coal rather than the combustible carbon material. Another study by Honaker et al. (2014) evaluated six coal and coal byproducts. The study stated that the rare earths are dispersed in the form of monazite, xenotime, and bastnaesite with the particle size range changing between 1-10 $\mu \mathrm{m}$ or smaller. On the other hand, Dai et al. (2012) divided rare earth's formation in coal into five categories: water-soluble, ion exchangeable, and associated with carbonates, organic matter, and aluminum-silicates. Huang et al. (2019) studied several Colombian coal samples to address the geologic mechanisms that lead to rare earth enrichment in Latin American coals. The study verified that rare earth element content appears to be strongly dependent on the chemical setting during the coal's formation. The study also suggested a strong and positive relationship between rare earth and ash content. Pan et al. (2019) studied coal fly ash samples collected from China and observed a relationship between particle size and rare earth enrichment, and according to their study, rare earth-enriched components increased as the particles get finer. Also, a strong relation between Si/Al ratio and rare earth elements was observed.

Coal is recognized as the second-largest energy source in the world and has massive global reserves (860 billion tonnes). The world's coal consumption is estimated to increase at a rate of $0.6 \%$ per annum, which generates a tremendous volume of tailings in the form of bottom ash, fly ash, and slag (Adiansyah et al., 2017; Fiket et al., 2018). A report published by the American Coal Ash Association (2017) stated that, in 2016, 38 million tons of fly ash were generated in the U.S, and only $60 \%$ of it was utilized. Therefore, using coal processing products for rare earth production 
can turn the waste of one industry into the feedstock of another, which eventually leads to a more circular economy (Das et al., 2018; Zhou et al., 2018). While focusing on the technical feasibility and economic viability of the extraction and recovery of REEs from coal and coal byproducts, it is equally crucial to ensure that the process is environmentally benign (Navarro and Zhao, 2014; Valkov et al., 2014). The environmental prospect of rare earth mining was not thoroughly considered until recent years. However, nowadays, the development of cleaner production becomes a significant subject, and environmental control is now an essential part of mine management and many other industries (Paul and Campbell, 2011).

\subsection{Environmental Impact of Rare Earth Extraction and Regulatory Stipulations}

As mentioned previously, rare earth mining faces challenges due to the detectable amount of radioactive matter (Pillai, 2007; Dutta et al., 2016). Both coal and traditional rare earth-bearing minerals being currently processed contain a considerable amount of thorium and uranium due to isomorphic substitution, which needs to be treated specially to prevent their interaction with groundwater and distribution to air. In some cases, in addition to isomorphic substitution, uranium, is also formed by epigenetic infiltration during the lignite-subbituminous stage of coalification (He, 2014; Ault et al., 2015; Qin et al., 2018; Findeiß, 2016; Kursun et al., 2018).

It was reported in a study that bastnaesite contains up to $0.3 \mathrm{wt} \%$ thorium dioxide and $0.09 \mathrm{wt} \%$ uranium dioxide, whereas the thorium and uranium contents in monazite can be as high as $20 \mathrm{wt} \%$ and $16 \mathrm{wt} \%$, respectively. Similarly, xenotime may contain up to $5 \mathrm{wt} \%$ uranium dioxide (Van Gosen et al., 2017). In other words, concentrations of ${ }^{238} \mathrm{U}$ and ${ }^{232} \mathrm{Th}$ in these minerals exceed 1000 $\mathrm{Bq} / \mathrm{kg}$ (Liu and Pan, 2011). As an unconventional resource for rare earth production, coal, also contains a significant amount of thorium and uranium either associated with elements in the coal itself or within the minerals that are part of coal formation (Cooper, 2005; Papastefanou, 2007; Department of Energy, 2017; Lange et al., 2017; Dai and Finkelman, 2018). Besides thorium and uranium, their decay products such as radium $(\mathrm{Ra})$ and radon $(\mathrm{Rn})$ are present in coal-related materials as well (United States Geological Survey, 1997). Moreover, many condensed elements with high volatility (i.e., $\mathrm{As}, \mathrm{Cd}, \mathrm{Cu}, \mathrm{Pb}$, etc.) are present on the surface of fly ash samples (Parzentny and Rog, 2019). The study conducted by Finkelman (1999) with approximately 7000 coal samples indicated that the average thorium and uranium concentrations in coal are 3.2 and 2.1 ppm, respectively. Another study conducted by Dai et al. (2014) also revealed a certain amount of uranium in coal samples taken from deposits located in China and Russia. According to a characterization study conducted by the United States Geological Survey (1997) with approximately 2,000 U.S. coal samples, the concentration of thorium and uranium has been found to mostly vary between 1 to $4 \mathrm{ppm}$ with the highest concentration value of $20 \mathrm{ppm}$ being reported in some cases. It was reported that uranium organically bounds within the coal and can also be associated with other minerals such as rare earth phosphates (Finkelman et al., 2018).

The rare earth element extraction processes can yield approximately 1.4 tons of radioactive waste in the form of solid, liquid, or gas for one ton of rare earth oxide (REO) (Jiabao and Jie, 2009; British Geological Survey, 2011; Navarro and Zhao, 2014). For example, at the Bayan Obo Mine, 
the largest mine in China, which produces rare earths as a secondary product of iron ore, 200 tons of thorium dioxide sludge are produced every year from 100 thousand tons of rare earth concentrates. When naturally-occurring radioactive materials are concentrated or released by human activities (i.e., mining), they are classified as technologically enhanced naturally occurring materials (TENORM) (Findeiß, 2016). If not regulated correctly, exposure to such elements may develop cancer risk for workers, severely damage surface vegetation, cause water pollution, and affect agricultural output.

In general, thorium and uranium-containing leaching wastes are generally neutralized with limestone before their disposal. If the radionuclide-containing waste is solid, it must be kept moist to minimize dust formation. Additionally, impoundment facilities are built with waterproof materials like high-density polyethylene (HDPE) to prevent their interference with soil and groundwater. Further, at the impoundment site, the radioactive element containing tailings are mixed with barium hydroxide $\left([\mathrm{BaOH}]_{2}\right)$ to precipitate radioactive elements (Findeiß, 2016). Another consideration is reducing the concentration of radioactive materials to less than $500 \mathrm{ppm}$, which is the internationally maximum allowed concentration limit to dispose of the waste without restriction. One way to do that is by mixing radioactive waste with other residues to lower the concentrations of thorium and uranium (Findeiß, 2016).

Various regulatory approaches exist to handle radioactive materials. The International Atomic Energy Agency (IAEA) has specific guidelines for these materials, which are used by many countries. According to a study conducted at this mine by Baotou Radiation and Environmental Institute, the radioactivity at the plant and in the soil was 32 and 1.7 times higher than the normal range, respectively. Typically, the uranium concentration in soil is between 0.3 to $11.7 \mathrm{mg} / \mathrm{kg}$, and the average radioactivity of ${ }^{234} \mathrm{U}$ is between 0.02 to $0.04 \mathrm{~Bq} / \mathrm{g}$ (Abdel-Sabour, 2014). Likewise, the thorium content in the leaching pond was around $5 \mathrm{mg} / \mathrm{L}$, while the thorium concentration in freshwater was 0.01 to $1 \mu \mathrm{g} / \mathrm{L}$ (Findeiß, 2016). In another study, it was reported that the radiation level in the Bayan Obo city area was in the range of 100-150 nGy/h which was $50 \%$ higher than the accepted value. This range increases to 600-2000 $\mathrm{nGy} / \mathrm{h}$ at mining sites. Similar high radiation activity results were reported in Wang and his coworkers' (2016) study. According to the measurements made in the vicinity of the Bayan Obo mine, the ${ }^{232} \mathrm{Th}$ activity in total suspended particulates in mining and smelting area was between $820-39$ and $720 \mu \mathrm{Bq} / \mathrm{m}^{3}$ respectively, which is significantly higher than the world's maximum acceptable limit of $0.5 \mu \mathrm{Bq} / \mathrm{m}^{3}$. For the purpose of cleaner production, China implemented the IAEA's safety standards as a national standard in 2003 to prevent ionizing radiation (Liu and Pan, 2011; Wang et al., 2017). Again in China, an environmental impact assessment approved by Environmental Protection Agency (EPA) is requested at non-uranium mining sites containing natural radioactive nuclides.

For thorium and uranium, radioactivity with less than $1 \mathrm{~Bq} / \mathrm{g}$ is considered not radioactive, and the waste is classified as exempt waste. Nonetheless, in the United Kingdom, $0.1 \mathrm{~Bq} / \mathrm{g}$ must be achieved for residental land use. Moreover, radioactive materials with less than $50 \mathrm{ppm}$ of uranium and thorium or any combination of the two are regarded as not radioactive by the U.S. Nuclear 
Regulatory Commission (NRC). Additionally, according to the World Health Organization (WHO), the maximum uranium level in drinking water can not exceed $15 \mu \mathrm{g} / \mathrm{L}$, and the maximum contaminant level set by the EPA is $20 \mu \mathrm{g} / \mathrm{L}$ (Rozmaric et al., 2009; Camacho et al., 2010). According to the Canadian Soil Quality Guidelines, the maximum allowable concentrations of these hazardous elements are $23 \mathrm{ppm}$ for agricultural and residential land use, $33 \mathrm{ppm}$ for commercial land use, and 300 ppm for industrial land use. (Abdel-Sabour, 2014). Waste within the allowed level is sent to a landfill, and a level greater than $100 \mathrm{~Bq} / \mathrm{g}$ must be buried (World Nuclear Association, 2021).

Furthermore, the chemical extraction of rare earths from monazite involves occupational radiation exposures in the range of $0.13-1 \mathrm{mSv}$ per ton depending on the concentration of monazite (Pillai, 2017). Other researchers studied thorium and uranium activity in a monazite mine in Spain and observed an activity range of 70-100 Bq/ kg and 40-60 Bq/ $/ \mathrm{kg}$ for thorium and uranium, separately (Garcia-Tenorio et al., 2018). The radioactivity and chemical characterization of Malaysian monazite processing residue was comprehensively investigated in Jaffary et al.'s (2019) study, which clearly pointed out that the radioactivity is above world permitted limits. Similarly, Aziman and Ismail (2020) conducted a study to emphasize the high radioactivity level of water leach residue generated after processing monazite in Malaysia. For these reasons, countries like Australia and the U.S. stopped rare earth production from monazite in the early 90s since it has the highest radioactive element concentration among other conventional rare earth-bearing minerals (Hedrick, 2000; Schuler et al., 2011). Similarly, in India, improved effluent treatment processes are applied and, the radionuclide concentration in the river, close to a mine site, is reduced subsequently (Paul and Pillai, 1991). On the other hand, in Malaysia, two rare earth processing plants were closed in 1992 due to the problem arising from disposing of a vast amount of naturally occurring radioactive material wastes (Al-Areqi et al., 2016). For more details regarding to the natural levels of radioactivity in rare earth solid waste are given in Table 3.

Table 3 Level of radioactivity in rare earth solid waste (modified from Liu and Pan (2011).

\begin{tabular}{cccccccc}
\hline \multicolumn{2}{c}{ Uranium $(\mathrm{Bq} / \mathrm{kg})$} & \multicolumn{2}{c}{ Ra-226 $(\mathrm{Bq} / \mathrm{kg})$} & \multicolumn{2}{c}{ Thorium $(\mathrm{Bq} / \mathrm{kg})$} & \multicolumn{2}{c}{$\begin{array}{c}\text { External } \gamma \text { Dose Rate } \\
(\mathrm{nGy} / \mathrm{h})\end{array}$} \\
\hline Average & Maximum & Average & Maximum & Average & Maximum & Average & Maximum \\
\hline 2,081 & 83,044 & 1,240 & 53,700 & 4786.3 & 85,600 & 3,249 & 48,344 \\
\hline
\end{tabular}

Even though the initial concentration of thorium and uranium in coal and coal byproducts is not high compared to their existence in traditional rare earth minerals, the radioactive elements can be substantially enriched along with the processing stages and may present in the form of solid, liquid, or gaseous wastes. It was stated that when coal is burned into fly ash, it creates a concentrated form of thorium and uranium with a concentration approximately 10 times higher than the initial level (Hvistendahl, 2007). Although the direct toxicity of thorium is low because of its stability under ambient temperature, the fine thorium powder is self-ignitable (Metaxas et al., 2003). The radionuclides may accumulate in the upper layer of the soils, form complexes with organics, and 
can be moved into water streams or air (Abdel-Sabour, 2014). When these toxic ions enter living organisms, such as the human body, they tend to accumulate in the liver, spleen, and marrow, where they precipitate in their hydroxide form (Metaxas et al., 2003). It is noteworthy that acid mine drainage (AMD) sludge has very low concentrations of thorium and uranium (Vass et al., 2019). Vass et al. (2019) studied several AMD sludge samples and reported an average of 5.67 and $4.46 \mathrm{~g} /$ ton thorium and uranium concentration, respectively. The radioactivity level of the radionuclides in coals from different regions are presented in Table 4 and the level of radioactivity in the solid waste generated from coal processing is given Table 5. Parzentny and Rog (2019) performed a detailed study on the role of mineral matter in concentrating thorium and uranium in coal and combustion residues, where the researchers also summarized the thorium and uranium contents of a wide variety of coal and coal-based samples worldwide (Table 6).

Table 4 Radioactivity level $(\mathrm{Bq} / \mathrm{kg}$ ) of the nuclides in coal in various countries (modified from World Nuclear Association (2021)).

\begin{tabular}{ccccc}
\hline Country & U-238 & Ra-226 & Ra-228 & Th-232 \\
\hline Australia & $8.5-47$ & $19-24$ & $11-64$ & $11-69$ \\
Brazil & 72 & 72 & 62 & 62 \\
China & \multicolumn{3}{c}{ Typically 10-25, may be up to 29,000 } \\
Germany & \multicolumn{3}{c}{ 10-25, } & \multicolumn{2}{c}{ 10-63, } \\
average 32 32 2 & average 21 \\
Greece & $117-390$ & $44-206$ & $9-41$ & NA \\
Hungary & $20-480$ & NA & $12-97$ & NA \\
Poland & 159 & NA & 123 & NA \\
Romania & 415 & 557 & NA & 170 \\
United Kingdom & $7-19$ & $8-22$ & NA & $7-19$ \\
United States & $6-73$ & $8.9-59$ & NA & $4-21$ \\
\hline
\end{tabular}

Table 5 Level of radioactivity $(\mathrm{Bq} / \mathrm{kg}$ ) in coal solid waste (modified from Liu and Pan (2011)).

\begin{tabular}{cccccccc}
\hline \multicolumn{2}{c}{ Uranium } & \multicolumn{2}{c}{ Ra-226 } & \multicolumn{2}{c}{ Thorium } & \multicolumn{2}{c}{$\begin{array}{c}\text { External } \gamma \text { Dose Rate } \\
(\mathrm{nGy} / \mathrm{h})\end{array}$} \\
\hline Average & Maximum & Average & Maximum & Average & Maximum & Average & Maximum \\
\hline 225 & 7600 & 326 & 92,178 & 91 & 910 & 162 & 987 \\
\hline
\end{tabular}


Table 6 Thorium and uranium concentration in the world's selected coal and coal-based materials (modified from Parzentny and Rog (2019)).

\begin{tabular}{|c|c|c|c|}
\hline Type of Material & Country/Area & $\begin{array}{l}\text { Thorium } \\
\text { (g/Mg) }\end{array}$ & $\begin{array}{c}\text { Uranium } \\
\text { (g/Mg) }\end{array}$ \\
\hline \multirow{7}{*}{ Raw coal } & Bulgaria & 6 & 5 \\
\hline & Poland & 3.2 & 1.9 \\
\hline & Poland & 2.3 & 1.9 \\
\hline & USA & 3.2 & 2.1 \\
\hline & USA & $1.5-5.9$ & $1.2-3.9$ \\
\hline & China & 5.84 & 2.43 \\
\hline & China & NA & $0.75-7207$ \\
\hline \multirow{6}{*}{$\begin{array}{c}\text { Feed Coal (the } \\
\text { coal before } \\
\text { burning) }\end{array}$} & Europe & 5.6 & 6.1 \\
\hline & Europe & $17-65$ & $5-29$ \\
\hline & Brazil & $14.09-17.04$ & $6.12-7.67$ \\
\hline & Poland & $1.1-2.6$ & $0.2-0.8$ \\
\hline & Spain & 5.6 & 6.1 \\
\hline & Turkey & 9 & 14 \\
\hline \multirow{9}{*}{ Fly ash } & Europe & 22.1 & 22.9 \\
\hline & Brazil & $33.5-42$ & $14.5-24.8$ \\
\hline & China & $5.8-50$ & $2.6-51.9$ \\
\hline & Poland & 23 & 10.6 \\
\hline & Poland & $7.6-19.3$ & $3.3-10.6$ \\
\hline & Spain & 22.1 & 22.9 \\
\hline & Turkey & 22 & 34 \\
\hline & USA & $14.0-28.0$ & $6.9-12.7$ \\
\hline & USA & $11.8-21.6$ & $6.72-10.4$ \\
\hline \multirow{8}{*}{ Bottom ash } & Europe & 20.6 & 19 \\
\hline & Australia & $15-42$ & $5-9.7$ \\
\hline & Brazil & $25.8-42.9$ & $9.3-16.7$ \\
\hline & China & $19.1-25$ & $8.5-379.2$ \\
\hline & Spain & 20.6 & 19 \\
\hline & Turkey & 15 & 19 \\
\hline & USA & $14.9-25.3$ & $0.9-9.7$ \\
\hline & USA & $13-25.3$ & $5.87-9.83$ \\
\hline
\end{tabular}




\subsection{Solution Purification Techniques to Remove Thorium and Uranium from Rare Earth Elements}

Processing rare earths requires complex separation techniques using a variety of chemical compounds. Typically, bastnaesite and monazite are first subjected to sample preparation and preconcentration, including size reduction, roasting, froth flotation, which is later followed by extraction with a strong acid (Yang et al., 2020). Fatty acids and hydroxamate-based collectors were found to be the most efficient for bastnaesite flotation, and benzoic acid has been found to be adequate in separating bastnaesite from monazite. Although it is not preferred these days, roasting the ore at $600-800{ }^{\circ} \mathrm{C}$ and leached it with $\mathrm{HCl}$ acid was another method to recover rare earth elements. An alternative approach was proposed by Yorukoglu and his coworkers' (2003) study to enhance the leaching ability of bastnaesite by the addition of thiourea $\left(\mathrm{CH}_{4} \mathrm{~N}_{2} \mathrm{~S}\right)$. In the case of monazite processing, typically, two methods are followed; sulfuric acid treatment or sodium hydroxide decomposition, which both require extremely high temperatures to activate the reactions (Yang, 2020). To reduce the thorium amount reporting to the pregnant leach solution, an alternative pretreatment was developed by Merrit (1990) which involves converting rare earth phosphates into rare earth oxysulfides by heating with $\mathrm{CaCl}_{2}$ or $\mathrm{CaCO}_{3}$ in a sulfurizing environment. Gravity separation techniques can be applied to recover relatively high-density rare earths from low-density gangues associated together. In a study conducted by Ozbayoglu and Atalay (2000), approximately 50\% recovery and 36\% REO product were achieved using a combination of the scrubbing-cycloning-multi gravity separation process. Similarly, recovery of rare earth elements can be enhanced by eliminating the magnetic gangue minerals via magnetic separators. Gao and Chen (2010) achieved approximately 80\% recovery using low-intensity and high-gradient magnetic separators.

Physical and chemical methods to recover rare earths are well-summarized (Ozbayoglu and Atalay, 2000; Kim and Osseo-Asare, 2012; Jordens, 2016; Kermer et al., 2016; Anderson, 2017; Suli et al., 2017; Zhang and Honaker, 2018; Honaker et al., 2019; Zhang and Honaker, 2019). However, during rare earths recovery, thorium and uranium are also enriched alongside rare earths. Especially after the leaching of rare earths with acids, such as sulfuric acid $\left(\mathrm{H}_{2} \mathrm{SO}_{4}\right)$, hydrochloric acid $(\mathrm{HCl})$, or nitric acid $\left(\mathrm{HNO}_{3}\right)$, thorium and uranium also dissolve, which in return, requires further purification of the leachate. Although many remediation solutions are known, only a few of them have been found feasible for a large-scale operation. For this study, efforts focused on hydrometallurgical purification techniques, namely precipitation, solvent extraction, and adsorption processes. In some cases, the combination of multiple methods is suggested to be used (Chellam and Clifford, 2002; Akkaya, 2013; Zhang and Zhao, 2016).

\subsubsection{Selective Precipitation}

Selective precipitation of elements by controlled $\mathrm{pH}$ is the most well-known method for the removal of certain elements from impure leach liquors. Besides, it is a simple and cost-effective operation (Monhemius, 1977; Zhou et al., 2018). The underlying mechanism of precipitation is 
reacting chemicals with ions to generate insoluble complexes, which, can be separated from aqueous solutions by filtration (Zhou et al., 2018). Precipitation is induced by reduction using hydroxides or sulfides (Hostetler and Garrels, 1962). It takes the advantage of different precipitation $\mathrm{pH}$ ranges for thorium, uranium, and rare earths (Vijayalakshmi et al., 2001; Silva et al., 2018).

It was stated in Kim and Ossae's (2012) study that the dissolved species of thorium, uranium and rare earth metals in acidic solutions may be separated by $\mathrm{pH}$ adjustment, in which thorium separation is expected to take place first. This statement also agrees well with other studies in literature indicating different precipitation $\mathrm{pH}$ ranges for thorium, uranium, and rare earths. As indicated in the study of Zhu et al. (2015), thorium's precipitation $\mathrm{pH}$ range is typically 2.5-5.5; following this, uranium and rare earths precipitation subsequently takes place. The precipitation $\mathrm{pH}$ range of uranium and rare earths is approximately 5.5-7 and 6.8-8, respectively (Langmuir, 1978; Zhu et al., 2015).

At the Bayan Obo Mine, the rare earth mineral, bastnaesite, is beneficiated using a combination of physical and chemical processes. After leaching, both rare earths and thorium are converted into their hydroxide forms using sodium hydroxide, and then they are leached with hydrochloric acid. Finally, thorium and iron are precipitated at a $\mathrm{pH}$ region of 4 to 5 . The solution containing substantial rare earths elements proceeds to further treatment for the recovery of individual rare earth elements (Navarro and Zhao, 2014; Wang et al., 2017). In Sichuan Mine, the second largest rare earth mine in China, the ore is roasted at a temperature higher than $500{ }^{\circ} \mathrm{C}$, where thorium and cerium are converted to $\mathrm{ThO}_{2}$ and $\mathrm{CeO}_{2}$. Later, thorium is separated from cerium with the caustic attack. At Mountain Pass Mine, bastnaesite is leached with hydrochloric acid, which also dissolves uranium along with rare earths. The dissolved uranium is then selectively precipitated using barium chloride or sodium sulfate (Findeiß, 2016).

Al-Areqi and his coworkers (2016) studied selective precipitation of thorium from Malaysian monazite using ammonia. In addition to the typical precipitation method induced by adjusting the solution $\mathrm{pH}$ with a basic chemical, oxalic acid has been employed to initially co-precipitate thorium and rare earths so as to separate them from uranium. The precipitate is subsequently subjected to selective dissolution to further separate thorium from rare earth using a hot sodium carbonate solution (Zhu et al., 2015). A similar process was followed by Amer et al. (2013), in which alkali breakdown of monazite was first achieved and followed by dissolution-oxalate precipitation to leave uranium in the solution. The precipitation efficiencies of rare earths and thorium reached $99 \%$. Oxalate precipitation reactions of rare earth elements, thorium, and uranium in sulfate media are given in the Equations (1-4). Later, thorium separation can be achieved by dissolving the precipitated solid in a carbonate-containing solution (i.e., $\mathrm{Na}_{2} \mathrm{CO}_{3}$ and $\mathrm{NaHCO}_{3}$ ) where thorium will create a soluble complex.

$$
\begin{aligned}
& R E E_{2}\left(\mathrm{SO}_{4}\right)_{3(a q)}+3 \mathrm{H}_{2} \mathrm{C}_{2} \mathrm{O}_{4(a q)}=R E E_{2}\left(\mathrm{C}_{2} \mathrm{O}_{4}\right)_{3(\mathrm{~s})}+3 \mathrm{H}_{2} \mathrm{SO}_{4(a q)} \\
& \mathrm{Th}\left(\mathrm{SO}_{4}\right)_{2(\mathrm{aq})}+2 \mathrm{H}_{2} \mathrm{C}_{2} \mathrm{O}_{4(\mathrm{aq})}=\mathrm{Th}\left(\mathrm{C}_{2} \mathrm{O}_{4}\right)_{2(\mathrm{~s})}+2 \mathrm{H}_{2} \mathrm{SO}_{4(\mathrm{aq})}
\end{aligned}
$$




$$
\begin{aligned}
& \mathrm{UO}_{2}\left(\mathrm{SO}_{4}\right)+\mathrm{H}_{2} \mathrm{C}_{2} \mathrm{O}_{4(a q)}=\mathrm{UO}_{2}\left(\mathrm{C}_{2} \mathrm{O}_{4}\right)+\mathrm{H}_{2} \mathrm{SO}_{4(a q)} \\
& \mathrm{UO}_{2}\left(\mathrm{C}_{2} \mathrm{O}_{4}\right)+3 \mathrm{H}_{2} \mathrm{SO}_{4(a q)}=\mathrm{UO}_{2}\left(\mathrm{SO}_{4}\right)+2 \mathrm{CO}_{2(a q)}+3 \mathrm{H}_{2} \mathrm{O}
\end{aligned}
$$

Double salt precipitation with sodium or sulfate can also be an alternative method; however, thorium's ability to form double salts is limited. Although some studies report thorium precipitation as high as $80 \%$, other studies are stating lower thorium precipitation (25-30\%) (Zhu et al., 2015). Therefore, this method varies depending on the operational conditions and ore characteristics.

\subsubsection{Solvent Extraction}

Solvent extraction has been the most extensively used method for purification purposes due to its capability to handle large volumes of aqueous solutions (Asselin et al., 1949; Hidayah and Abidin, 2017). It has also been an effective technique to remove hazardous matters from rare earths (Brown and Sherrington, 1979).

One of the crucial parameters in the solvent extraction process is the type and concentration of the extractant (organic chemical) because the efficiency of the process depends on the extractant's ability to transfer metal ions between two immiscible phases, aqueous and organic (Hidayah and Abidin, 2017; Zhou et al., 2018). Organic phosphorus acid, carboxylic acid, tertiary and quaternary amines, and sulphoxide are some of the commercially used extractant types which have been proved to be effective for the separation of radioactive elements while present with rare earths (Gupta et al., 2002; Belova et al., 2015; Zhu et al., 2015; Giri and Nath, 2016; Kuang et al., 2017). However, most of these extractants are also effective for the recovery of rare earths. Therefore, it is crucial to develop a unique experimental scheme for the desired selectivity and separation efficiency.

Many studies have been conducted to investigate the impact of different extractant types, diluents, and operating conditions on the removal of thorium and uranium from traditional rare earthbearing minerals (Nasab, 2014; Lu et al., 2016; Samsonov et al., 2015; Wang et al., 2017). One of the earliest studies in this field was conducted by Hughes and Singh (1980), who used a secondary amine to separate thorium from monazite. Palmieri (1987) explored the possibility of separating uranium using chelating reagents. Likewise, Gupta et al. (2002) investigated thorium and uranium separation from rare earths using Cyanex 923. On the other hand, Amaral and Morais (2010) studied the removal of thorium and uranium from sulfuric leach liquor generated from monazite leaching using a mixture of primary and tertiary amine (Primene JM-T and Alamine 336) extractants. The expected reactions between primary amine and thorium, and tertiary amine and uranium are given in Eqs. 5 and 6, separately.

$$
\begin{aligned}
& 4 \mathrm{RNH}_{2} \mathrm{H}^{+} \mathrm{HSO}_{4}^{-}+\left(\mathrm{Th}\left(\mathrm{SO}_{4}\right)_{4}\right)^{-}=\left(\mathrm{RNH}_{2} \mathrm{H}^{+}\right)_{4}\left(\mathrm{Th}\left(\mathrm{SO}_{4}\right)_{4}\right)^{4-}+4 \mathrm{HSO}_{4}^{-} \\
& 4 \mathrm{R}_{3} \mathrm{NH}^{+} \mathrm{HSO}_{4}^{-}+\left(\mathrm{UO}_{2}\left(\mathrm{SO}_{4}\right)_{3}\right)^{4-}=\left(\mathrm{R}_{3} \mathrm{NH}^{+}\right)_{4}\left(\mathrm{UO}_{2}\left(\mathrm{SO}_{4}\right)_{3}\right)^{4-}+4 \mathrm{HSO}_{4}^{-}
\end{aligned}
$$


Furthermore, Nasab et al. (2011) studied thorium removal using Cyanex 272 and Cyanex 302 as the extractants. Conventional extractants such as di-Ethylhexyl phosphoric acid (D2EHPA) and tributyl phosphate (TBP) are also indicated as effective extractants for the purpose of thorium and uranium separation. However, the use of TBP is restricted to the extraction from nitrate and chloride media (Gupta et al., 2002). Purification of impurities using TBP in kerosene also required multi-stage extracting and stripping (Al-Areqi et al., 2015). Multiple contacts between aqueous and organic solutions may increase the extraction efficiency; however, the organic extractant tends to be lost into the aqueous phase after a long contact between aqueous and organic phases.

When the extraction is completed, the extractant is stripped by mixing the solution with acids. This removes ions from the organic phase, draws them into the aqueous acids, and regenerates the extractant (Jorjani and Shahbazi, 2016). In some cases, a combination of acid and precipitation stripping is applied to recover the organic extractant (Findeiß, 2016). To further recycle the used organic phase, ammonium nitrate solution and deionized hot water can be used to remove undesirable elements, such as calcium, iron, magnesium, and phosphorus (Jorjani and Shahbazi, 2016). A schematic representation of solvent extraction is given in Figure 1.

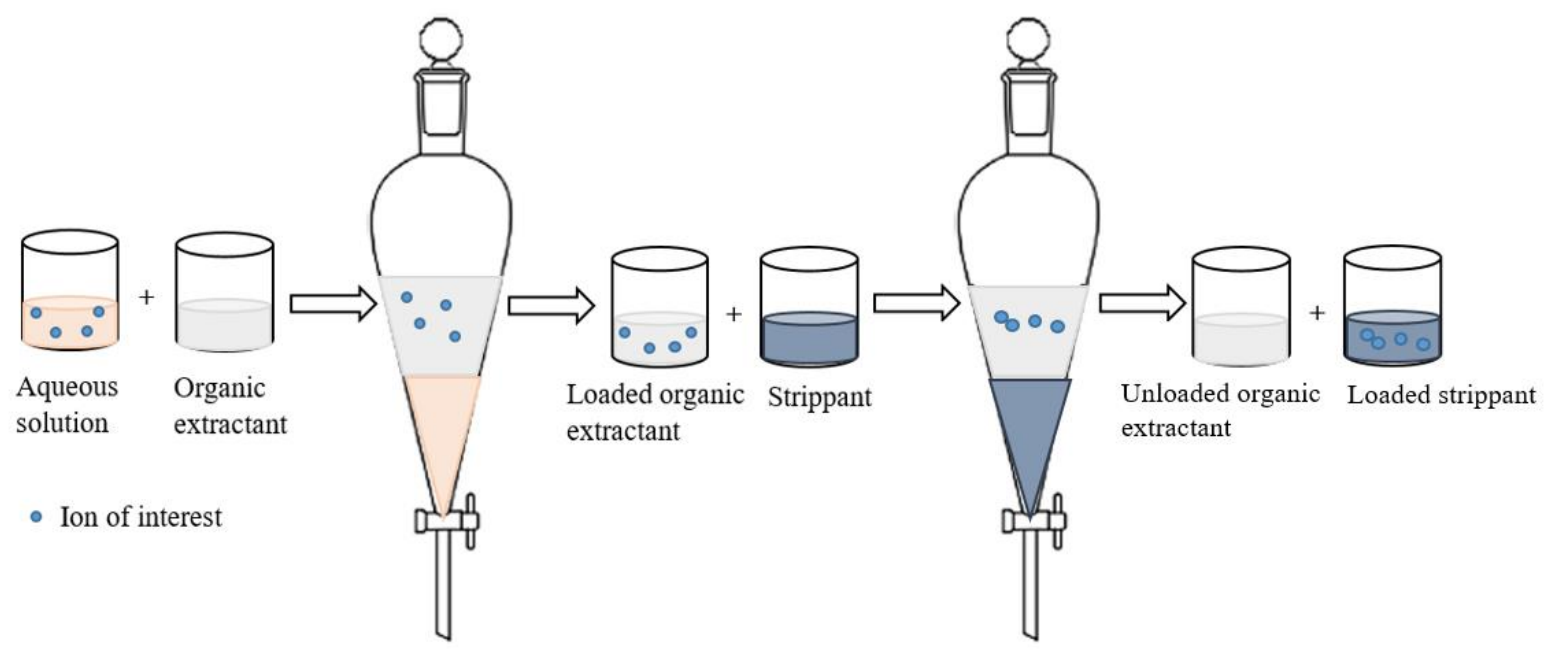

Figure 1 Schematic representation of solvent extraction tests.

\subsubsection{Solid-Phase Separation}

Separation using solid materials is heavily used in metal separations, hydrometallurgy, water and wastewater treatment, removal, and recovery of metal ions (Sani et al., 2015). Ion exchange and adsorption processes are two very similar solid-liquid separation techniques. Ion exchange is a process in which dissimilar ions with the same charge are exchanged between a solution and a solid (Hidayah and Abidin, 2017). The exchange reaction between ions in solution and ions attached to the matrix is generally reversible. On the other hand, adsorption can be explained by electrical attraction to the solid surface, and it is the net accumulation of matter at the interface between solid and aqueous phases (Jorgensen, 1989). 
Recently, the application of adsorption using porous solid sorbent has been gradually increased due to its high efficiency, easy handling, low maintenance, and operating costs over other techniques (Liu et al., 2013; Nandanwar et al., 2016). It has an advantage over the conventionally used solvent extraction mechanism by eliminating the consumption of organic extract and reducing environmental pollution that organic chemicals may create (Xiong et al., 2020). Adsorption has also been used for fuel processing containing thorium and uranium (Chen et al., 2016).

Sorbents are available in various material forms, such as natural and synthetic organic, inorganic, or composite materials. Their surfaces are often heterogeneous, and bonding energies may vary from one site to another. Materials like zeolite, apatite, activated carbon, calcined phosphate, oxides, and hydroxides are examples of some highly abundant sorbents (Ladeira and Goncalves, 2007). Especially silica-based materials have suitable physical, chemical, and thermal stability. There are plenty of studies about thorium and uranium adsorption on different materials (Aksoyoglu, 1989; Badei et al., 1992; Milonjic et al., 1992; Qadeer et al., 1992; Jain et al., 2001; Kilislioglu and Bilgin, 2003; Humelnicu et al., 2006; Sikalidis et al., 2008; Wang et al., 2012; Liu et al., 2013; Kaynar et al., 2015). Even though several studies were performed to examine the possible application of sorbents to remove naturally occurring heavy metals and radioactive materials, predominantly, these studies were performed for decontamination of polluted waters rather than rare earth production (Obaid et al., 2018). Doi et al. (1975) conducted a comprehensive study about uranium adsorption on a wide variety of sorbents such as peat, ferric oxide, clay minerals, zeolite, calcite, and apatite. The highest uranium uptake on peat was observed at a $\mathrm{pH}$ range of 3.8-8.5. It was noticed that the humic acid in the peat was causing uranium adsorption, and as the coalification progressed, the adsorption of uranium decreased in the order of peat, lignite, bituminous coal, and anthracite. Khalili and Al-Banna (2015) also studied thorium and uranium adsorption by humic acid. Sikalidis et al. (2008) studied thorium and uranium adsorption by clay minerals, montmorillonite, and vermiculite. Alternatively, Kutahyali and Eral (2010) investigated the adsorption of thorium and uranium on activated carbon. Fasfous and Dawoud (2012) performed a thorough study about the adsorption of uranium by multiwalled carbon nanotubes (CNT) and they also examined the kinetic and thermodynamic aspects of the adsorption process. Among all the carbon-based materials (i.e., carbon nanotubes, graphene, carbon fibers), CNT is more favorable due to its high specific surface area (Yadav et al., 2019). Further, Monji et al. (2016) studied the adsorption of uranium ions in the presence of lanthanides using a byproduct of the agricultural industry. Considering the immense quantities of agricultural waste, their use for the adsorption of ions substantially benefits the environment.

Sometimes the sorbent needs surface modification to enhance its adsorption capability. The modified material is called synthetic sorbent, which is more stable compared to its natural form (Ullah et al., 2012). Zou et al. (2009) studied uranium removal by manganese oxide-coated zeolite samples. On the other hand, Metaxas et al. (2003) and Baybas and Ulusoy (2011) focused on thorium adsorption using different zeolite and clay samples in addition to investigating the effect of the modified (pretreated) samples. In a similar study with activated carbon, Yakout (2017) stated 
that uranium adsorption onto $\mathrm{KOH}$-modified activated carbon is more selective than thorium adsorption. Most recently, Xiong et al. (2020) studied membrane-based separation using a novel sorbent (covalent organic frameworks, COFs) with high adsorption capacity in order to separate thorium from uranium and rare earths. Adsorption via membrane is known to consume less energy, and it is easy to operate as well.

The adsorption process depends on the chemical nature of both fluid and solid. Also, it is highly affected by the available surface area and pore volume of the adsorbent (Couper et al., 2009). Besides, the selectivity depends on many other factors such as the particle size of the absorbent, $\mathrm{pH}$ of the solution, contact time, nature of the solution, etc. The $\mathrm{pH}$ plays an important role in sorption processes because it can influence the aqueous chemistry of the ions, determines the hydrolysis products and the properties of the active sites of the sorbent (Faghihian et al., 2004; Cheira et al., 2017). It also affects the external charge of the adsorbent which results in a change in the electrostatic interactions. It was seen that the capacity of uranium absorption increases with increasing $\mathrm{pH}$ and reaches a maximum value at a $\mathrm{pH}$ range of 2.0 to 3.5 (Cheira et al., 2017). In another report published by the U.S. Environmental Protection Agency (1999), it was stated that when carbonate ions are present, the adsorption of uranium reaches its maximum in the $\mathrm{pH}$ range 5 to 8 and decreases as the $\mathrm{pH}$ continues to increase. Particle size distribution is another critical factor due to its influence on the mass transfer rates. Mass exchange rates increase with decreasing particle size (Kammerer et al., 2011; Monji et al., 2016). An increase in adsorption capacity is expected when the particles are finer since the effective surface area increases as the size decreases (Zou et al., 2009). The pore size distribution also plays a significant role in the diffusion of the solutes onto the sorbents.

Following adsorption, desorption is performed by either elevating the temperature, reducing pressure, or washing with a suitable reagent. The desorbed material may be recovered as a valuable product or discarded as waste (Couper et al., 2009). 


\section{CHAPTER 3}

\section{EXPERIMENTAL AND METHODLOGY}

\subsection{Samples and Materials}

Synthetic leach solutions containing rare earth elements, thorium, uranium, and major metals were prepared and used throughout the study. Standard Inductively Coupled Plasma (ICP) solutions with $1000 \mathrm{ppm}$ of rare earths, thorium, uranium was purchased from Ricca Chemical while respective metal chlorides with a grade higher than 99\% were purchased from Alfa Aesar.

Various alkaline salts (i.e., sodium hydroxide $(\mathrm{NaOH})$, potassium hydroxide $(\mathrm{KOH})$, ammonia hydroxide $\left(\mathrm{NH}_{4} \mathrm{OH}\right)$ ) were purchased from Merck and Fisher Scientific for selective precipitation tests. Hydrochloric acid $(\mathrm{HCl})$ and sodium hydroxide with analar grade were used throughout the study for $\mathrm{pH}$ adjustment. The Oakton $450 \mathrm{pH}$ meter was used to measure the solution $\mathrm{pH}$, which

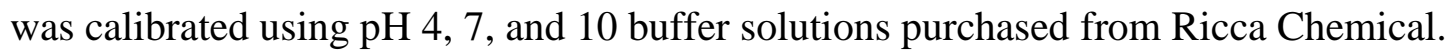

Tri-butyl phosphate (TBP) and kerosene were purchased from Fisher Scientific and Sigma Aldrich, respectively, and used as extractant and diluent for solvent extraction tests. Adsorption studies were performed as well, during which zeolite samples with different particle sizes were purchased from both KMI Zeolite Inc. and Heiltropfen Lab LLP.

Deionized water was used at all stages of the study, and experiments were performed at room temperature unless otherwise noted. During each experiment, representative samples taken from the aqueous and solid samples that were generated from various stages were subjected to rare earth elements and major metal analyses using Inductively Coupled Plasma-Optical Emission Spectroscopy (ICP-OES), and thorium and uranium analysis using Inductively Coupled PlasmaMass Spectroscopy (ICP-MS). ICP techniques were used due to their ability to detect trace elements with low detection limits and high sensitivity. While ICP-MS analyses were provided by West Virginia University National Research Center for Coal and Energy (WVU NRCCE), ICPOES analyses were provided by the University of Kentucky. Except for XRF, particle size distribution, and BET nitrogen adsorption analyses, all other characterization testing was performed at WVU Shared Research Facility. A detailed description of each analysis is given in part 3.4.

\subsection{Sample Preparation}

The initial feedstock material used at the earlier stages of the study was obtained from a coal preparation plant operating in Southern West Virginia. The coarse coal refuse sample was subjected to various unit operations at the pilot coal processing plant located in Providence,

Kentucky, to produce high-grade rare earths. Crushing, leaching, solvent extraction, and stripping were applied using di-(2-Ethylhexyl) phosphoric acid (D2EHPA) and $6 \mathrm{~mol} / \mathrm{L}$ hydrochloric acid as the extractant and stripping agent, respectively. An acidic strip solution was generated during 
the process and used for the exploratory tests that aimed to separate thorium and uranium from rare earths.

Later, synthetic solutions were prepared following the elemental composition of the strip solution produced at the pilot plant. The solution was prepared freshly before each test to avoid any sudden compositional change which may occur over time. Calculated volume from $1000 \mathrm{ppm}$ standard ICP solutions of REEs, Th, and U were taken by pipettes to prepare synthetic leach solution. Six $\mathrm{mol} / \mathrm{L}$ hydrochloric acid medium was maintained during the synthetic feedstock preparation. The elemental composition of the synthetic feedstock is provided in Table 7. As seen, the concentration of total rare earth elements is approximately $37 \mathrm{mg} / \mathrm{L}$ while the concentration of thorium and uranium is 0.5 and $0.9 \mathrm{mg} / \mathrm{L}$, separately. The content of major metals (i.e., Al, Ca, Fe, K, and Mg) is around $74 \mathrm{mg} / \mathrm{L}$.

Table 7 Elemental composition of synthetic feedstock solution.

\begin{tabular}{ccccccccc}
\hline Element & $\mathrm{Sc}$ & $\mathrm{Y}$ & $\mathrm{La}$ & $\mathrm{Ce}$ & $\mathrm{Pr}$ & $\mathrm{Nd}$ & $\mathrm{Sm}$ & $\mathrm{Eu}$ \\
\hline Concentration $(\mathrm{mg} / \mathrm{L})$ & 0.00 & 3.09 & 1.91 & 14.47 & 1.90 & 9.03 & 2.67 & 0.47 \\
\hline Element & $\mathrm{Gd}$ & $\mathrm{Tb}$ & $\mathrm{Dy}$ & $\mathrm{Ho}$ & $\mathrm{Er}$ & $\mathrm{Tm}$ & $\mathrm{Yb}$ & $\mathrm{Lu}$ \\
\hline Concentration $(\mathrm{mg} / \mathrm{L})$ & 2.01 & 0.10 & 0.82 & 0.09 & 0.33 & 0.00 & 0.14 & 0.01 \\
\hline Element & $\mathrm{Th}$ & $\mathrm{U}$ & $\mathrm{Al}$ & $\mathrm{Ca}$ & $\mathrm{Fe}$ & $\mathrm{K}$ & $\mathrm{Mg}$ & \\
\hline Concentration $(\mathrm{mg} / \mathrm{L})$ & 0.50 & 0.86 & 1.99 & 55.20 & 12.85 & 0.82 & 2.86 & \\
\hline
\end{tabular}

\subsection{Methodology}

\subsubsection{Preliminary Selective Precipitation Tests}

A number of preliminary studies were performed to assess the potential of using different separation techniques to remove thorium and uranium from rare earths. Selective precipitation tests were first conducted to selectively precipitate out thorium, uranium, and rare earth elements according to their distinctive precipitation behaviors at different $\mathrm{pH}$ values.

A 200-ml feedstock solution with an initial $\mathrm{pH}$ value of zero was used for the experimental testing. $2 \mathrm{~mol} / \mathrm{L}$ sodium hydroxide $(\mathrm{NaOH})$, potassium hydroxide $(\mathrm{KOH})$, and ammonium hydroxide $\left(\mathrm{NH}_{4} \mathrm{OH}\right)$ were used to incrementally increase the $\mathrm{pH}$, respectively. The $\mathrm{pH}$ value was elevated gradually by adding alkaline solution in a step-wise manner followed by three-min conditioning using a mechanical stirrer. Since the addition of hydroxides augments the hydrolysis and above a certain concentration, it results in precipitation (Tomazic et al., 1962), each addition of alkaline salt was monitored carefully to prevent sudden increments (Figure 2A). When significant precipitation was visually observed, the test was paused, and the solution was subjected to settling for a sufficient amount of time and subsequent filtration using $0.45 \mu \mathrm{m}$ pore-sized filter paper (Figure 2B). Afterward, precipitated solids were dried and weighed. A detailed flowsheet governing selective precipitation tests is shown in Figure 3. 


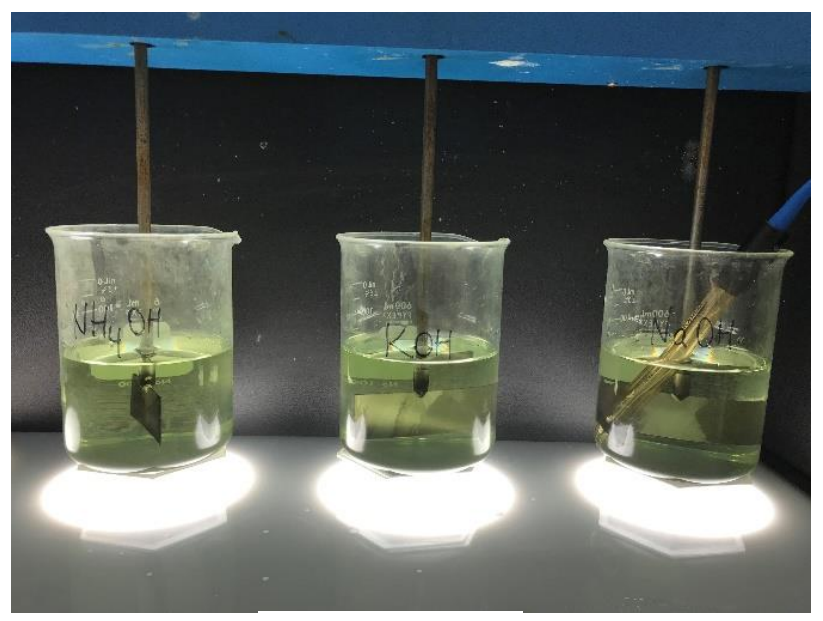

(A)

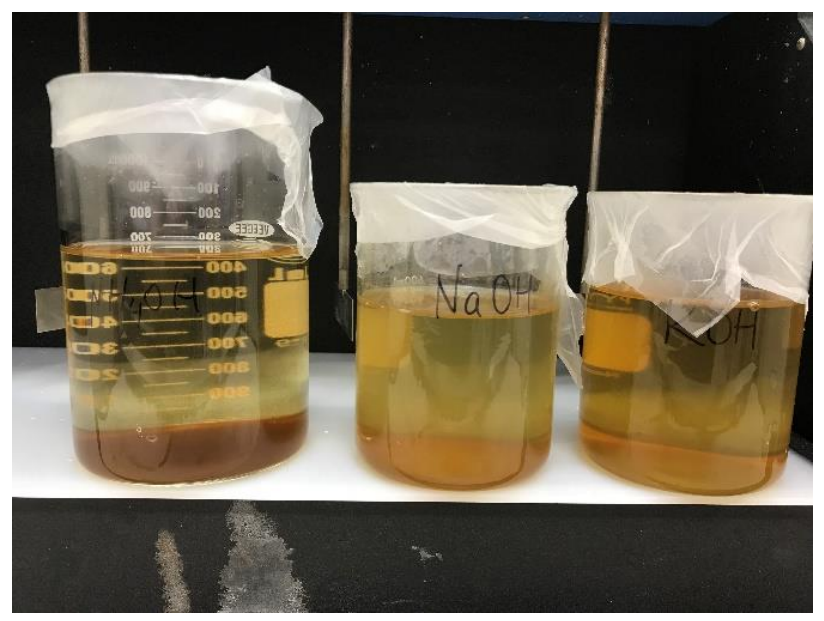

(B)

Figure 2 Pictures illustrating the experimental procedures of selective precipitation tests. (A) $\mathrm{pH}$ measurement, (B) Settling after precipitation.

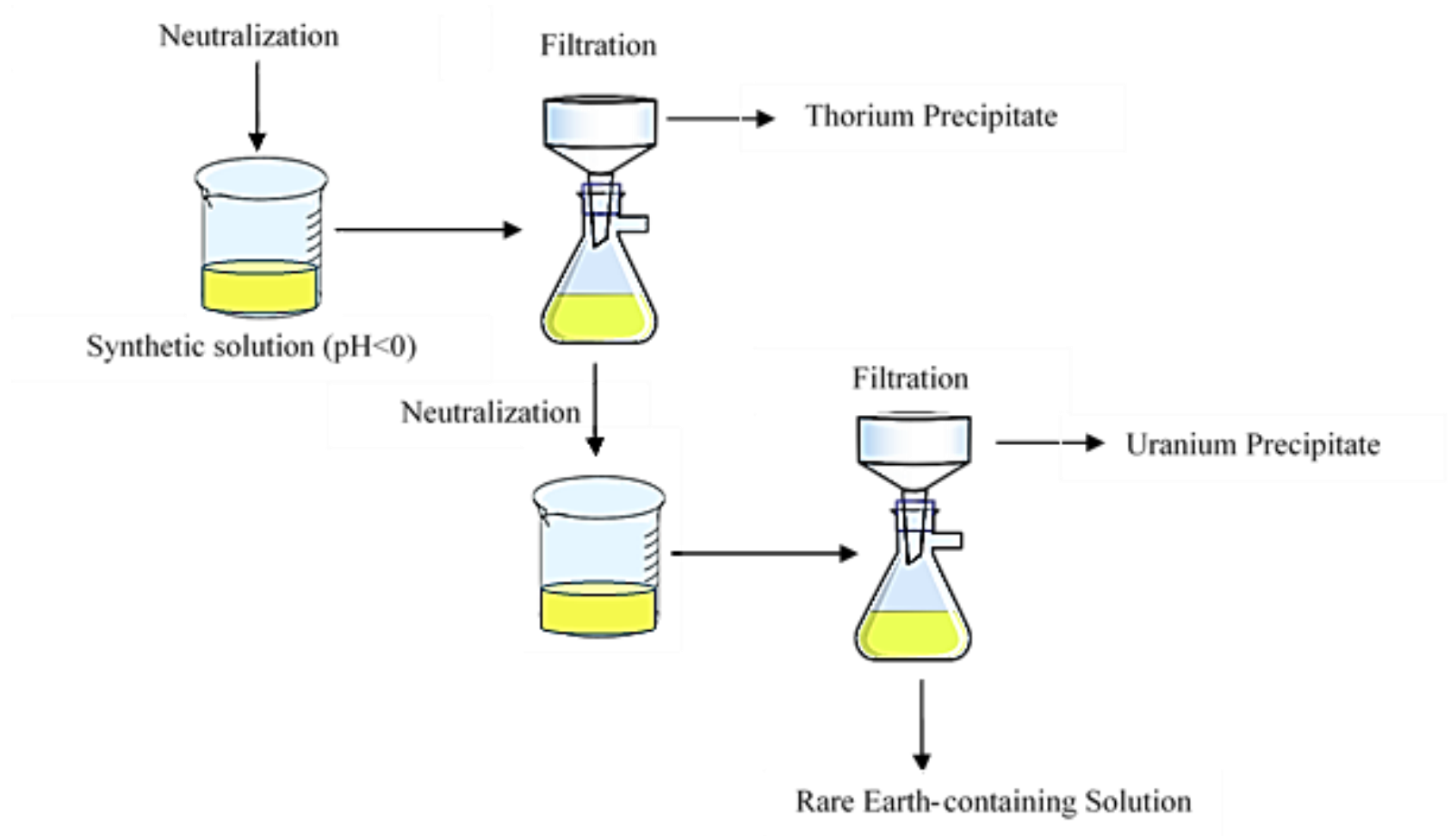

Figure 3 Experimental flowsheet followed during selective precipitation tests. 


\subsubsection{Preliminary Solvent Extraction Tests}

In addition to precipitation, solvent extraction tests were continued to explore its possibility of separating radioactive elements from rare earths. Tri-butyl phosphate was selected for the study due to its capability to selectively extract thorium and uranium under controlled operating conditions (Palmieri, 1987; Habashi, 1997; Zhang et al., 2016). Two sets of solvent extraction tests were designed and performed during the study, and the corresponding flowsheet is given in Figure 4.

Each test was conducted with a 50-ml synthetic feedstock solution at an initial $\mathrm{pH}$ value of 2.5 . As seen from Figure 4, the solvent extraction test consisted of two parts, with the first and second parts targeting uranium and thorium removal, separately. To initially separate uranium, $5 \mathrm{v} \%$ TBP dispersed in a kerosene solution was used as the extracting organic phase. Kerosene was chosen as the diluent to enhance the extraction capability of TBP and reduce the emulsification of the extractant (Anitha et al., 2014). Mixing of the organic and aqueous phases was performed using a mechanical stirrer, then the phases were separated using a separation funnel (Figure 5). A $2 \mathrm{~mol} / \mathrm{L}$ $\mathrm{HNO}_{3}$ solution was used at both the scrubbing and stripping stages. A scrubbing circuit was engaged after the extraction phase to wash impurities from the organic phase before the final purification stage.

The aqueous phase obtained from the uranium extraction step was subsequently sent for the thorium extraction, and 40 v\% TBP was used accordingly. Deionized water was used for the thorium stripping. Both sets of the solvent extraction tests were conducted under the same conditions except for the number of stages applied to extraction, scrubbing, and stripping. For the first set of exploratory tests, each unit process (extraction, scrubbing, stripping) was conducted as a single stage, while two stages were conducted for each of the aforementioned processes during the second set of tests. Test results obtained were used to compare the separation efficiencies resulting from various extraction stages, scrubbing, and stripping. Detailed operating procedures for the exploratory solvent extraction tests are summarized in Table 8.

Table 8 Operating conditions for exploratory solvent extraction tests.

\begin{tabular}{ccc}
\hline & Uranium Separation & Thorium Separation \\
\hline $\mathrm{pH}$ & 2.5 & 2.5 \\
Extractant $(\mathrm{v} / \mathrm{v})$ & $5 \% \mathrm{TBP}$ & $40 \% \mathrm{TBP}$ \\
Scrubbing agent & $2 \mathrm{~mol} / \mathrm{L} \mathrm{HNO}_{3}$ & - \\
Stripping agent & $2 \mathrm{~mol} / \mathrm{L} \mathrm{HNO}_{3}$ & DI water \\
O/A ratio at extraction & $1: 1$ & $2: 1$ \\
O/A ratio at scrubbing & $5: 1$ & - \\
O/A ratio at stripping & $2: 1$ & $2: 1$ \\
Retention time (min) & 20 & 20 \\
\hline
\end{tabular}




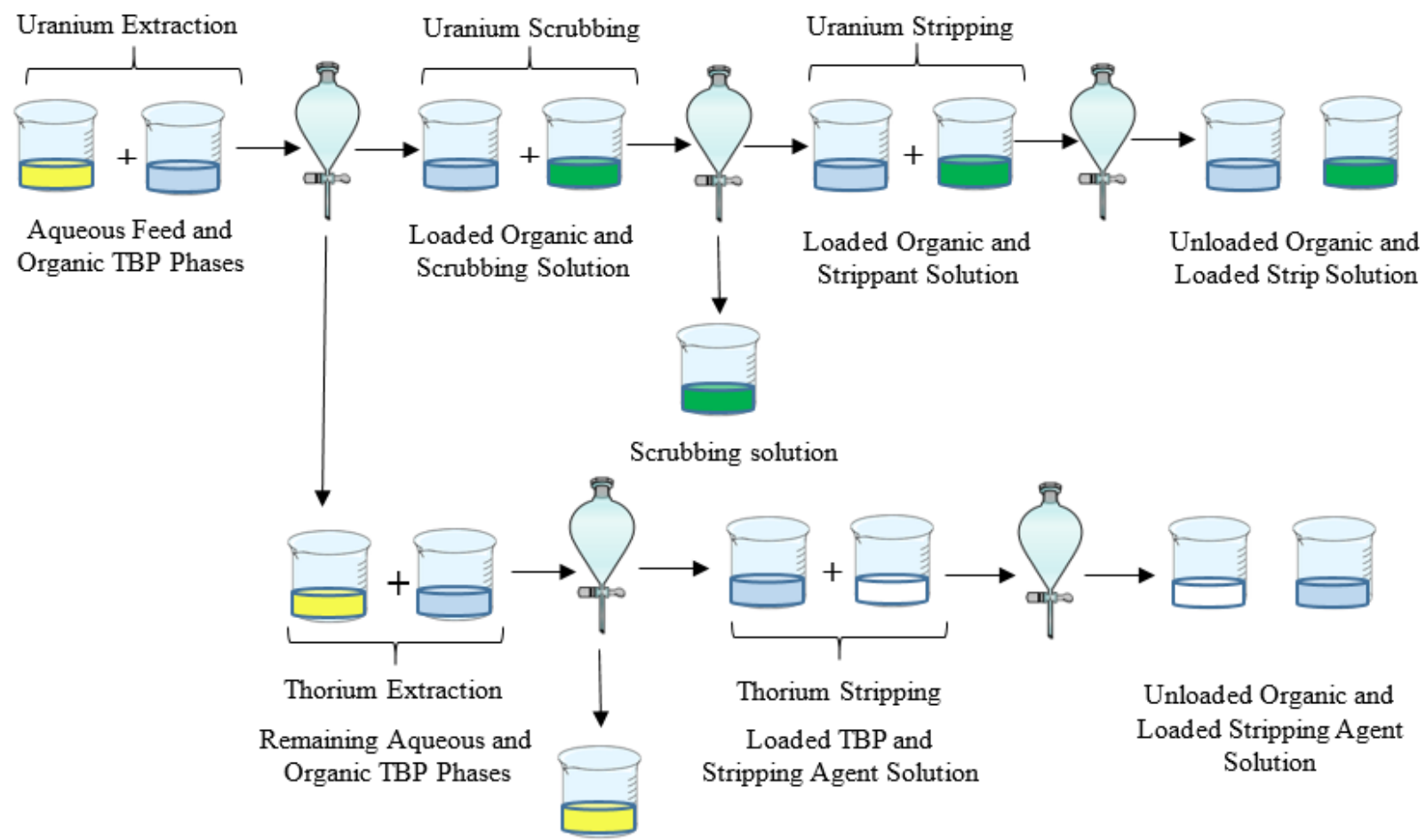

Rare earth-enriched solution

Figure 4 Experimental flowsheet followed during the exploratory solvent extraction tests.
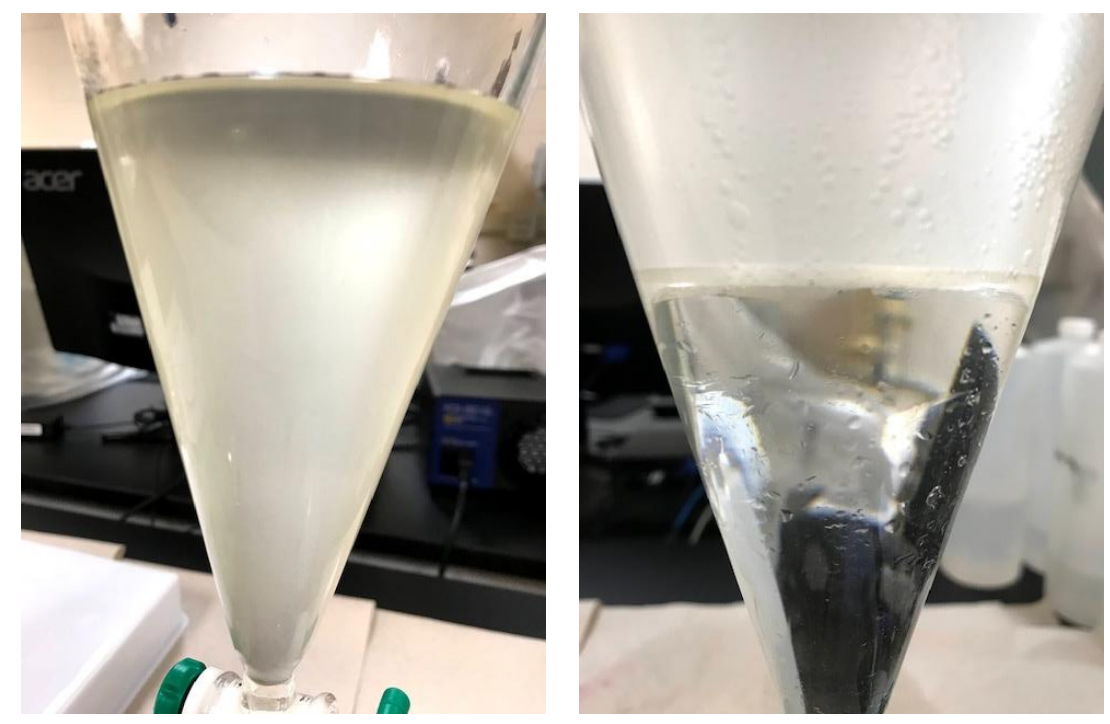

Figure 5 Separation between aqueous and organic phase during solvent extraction tests. 


\subsubsection{Modified Experimental Protocol}

A modified experimental protocol (Figure 6) was developed based on the test results obtained from the previous experimental work. The new experimental flowsheet consisted of one-stage selective precipitation followed by two-stage solvent extraction that aimed to achieve enhanced separation between thorium, uranium, and rare earths. A $2 \mathrm{~mol} / \mathrm{L}$ sodium hydroxide solution was used to gradually increase the $\mathrm{pH}$ to a value of around 4.85 that corresponded to a region under which solid precipitation was first observed. The tests were designed in a manner that precipitation aimed to selectively precipitate out thorium while the remaining filtrate loaded with uranium and rare earth elements was used as the feedstock for subsequent solvent extraction. Each solvent extraction test was performed with a 100-ml filtrate solution obtained from prior selective precipitation. Extraction and stripping were both carried out in two stages. In addition, various parameters, such as type and concentration of extractant, feed solution $\mathrm{pH}$, organic to aqueous ratio $(\mathrm{O} / \mathrm{A})$, and type and concentration of strippant, were evaluated to optimize the solvent extraction stage. Both rare earth-enriched (aqueous phase 2 in Figure 6) and uranium-enriched (aqueous phases 3 and 4 in Figure 6) streams were produced at the end of each solvent extraction test. Representative aqueous samples were taken from each step for rare earth, thorium, and uranium analysis using both ICPMS and ICP-OES.

\subsubsection{Experimental Design for Modified Experimental Protocol}

Experimental design methodologies were used to guide the design of various separation techniques applied in this study. For this purpose, a Box-Behnken experimental design technique based on response surface methodology (RSM) was used to determine the number of tests that need to be conducted. The design technique also ensures that the tests to be performed are sufficient to evaluate the significance of every influential operating parameter. Detailed descriptions of the experimental designs are given in the following sections.

The modified experimental protocol (Figure 6) was divided into two subsets of experimental design. Each subset has a fixed combination of extractant and strippant, as shown in Figure 7(A) and Figure 7(B), separately. It can be seen that three or four operating parameters, three levels of each, will be investigated during each subset study. The levels of each parameter have been determined based on prior studies conducted in a similar field (Menzies and Rigby, 1961; Nasab et al., 2011; Biswas et al., 2013; Xie et al., 2014; Jha et al., 2016). The retention time was fixed at $10 \mathrm{~min}$. A total of 15 tests and 27 tests were conducted for the first and second subset, respectively, with the only difference being the stripping agent. The equation used to determine the number of tests for each subset is:

$$
N=2 k(k-1)+C_{0}
$$

where $\mathrm{N}$ is the number of tests required for the experimental design, $\mathrm{k}$ is the number of variables to be studied $(\mathrm{k}=3), \mathrm{C}_{0}$ is the number of center points $\left(\mathrm{C}_{0}=3\right)$. Detailed test order and conditions for the two subsets of experiments are shown in Table 9 and Table 10, respectively. Adequate separation is obtained in conditions of low extraction of one component and high extraction of the 
other. One component tends to stay in the organic phase, and the other in the aqueous phase. This is achieved when the chemical nature of the compounds is significantly different.

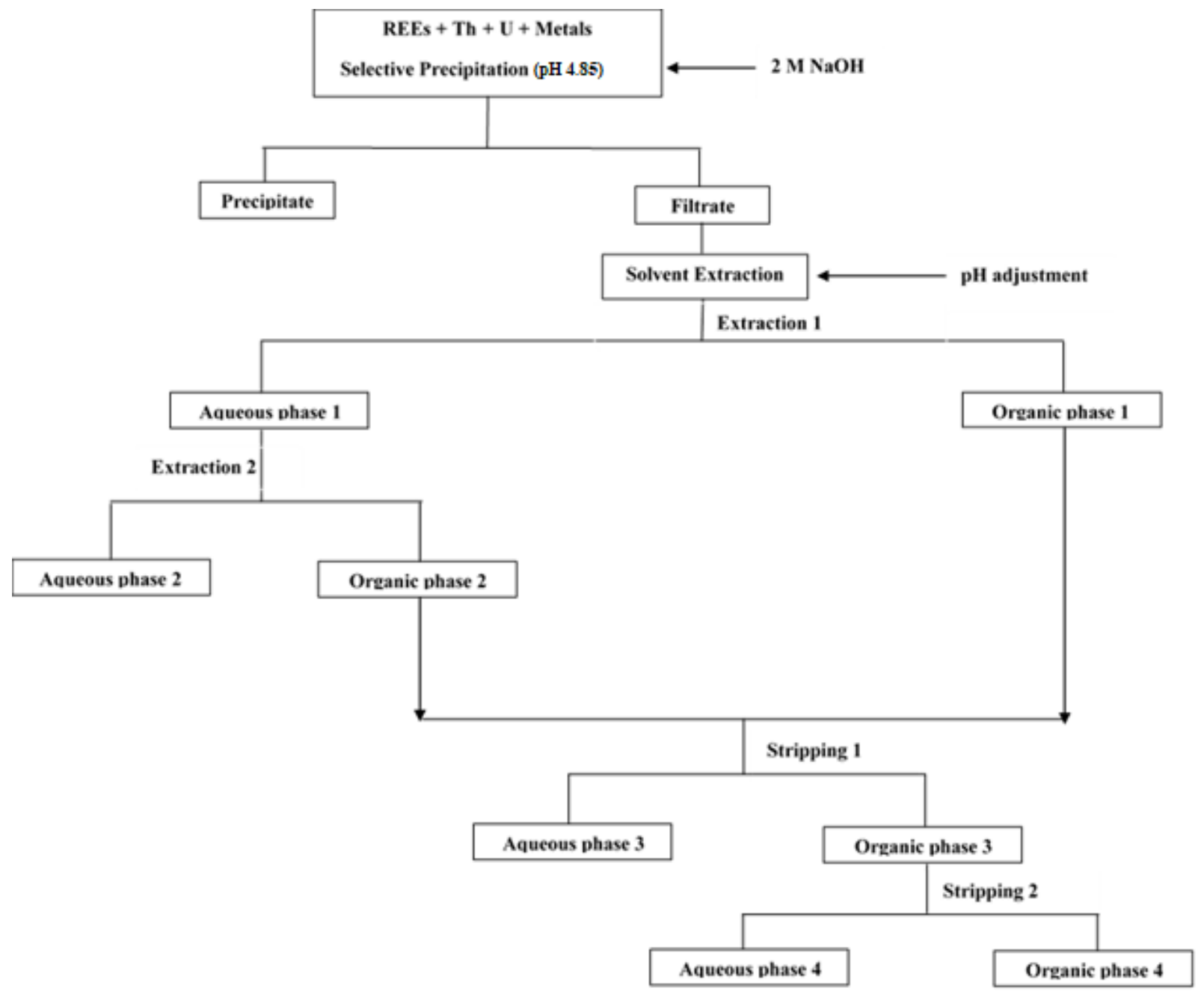

Figure 6 A modified experimental protocol developed for enhanced separation of thorium, uranium, and rare earths. 


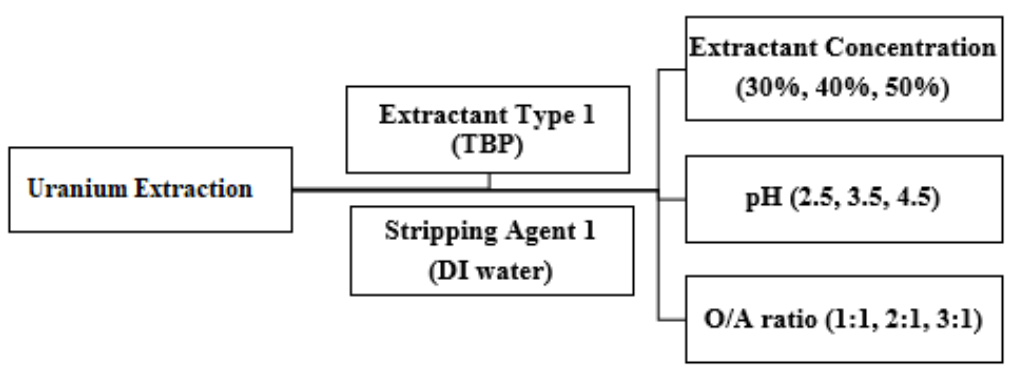

(A)

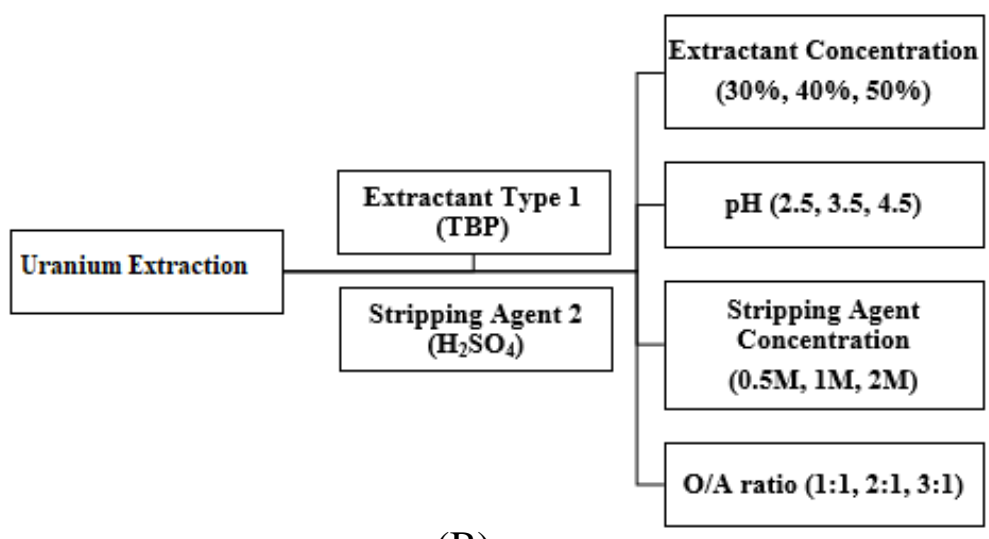

(B)

Figure 7 Experimental designs for uranium separation using TBP as the extractant: (A) DI water as the strippant; (B) $\mathrm{H}_{2} \mathrm{SO}_{4}$ as the strippant.

Table 9 Test order and conditions of the solvent extraction stage following the first subset of the modified experimental protocol.

\begin{tabular}{cccc}
\hline Test No. & Extractant Conc. (v\% TBP) & $\mathrm{pH}$ & Organic/Aqueous Ratio \\
\hline 1 & & 2.5 & 1 \\
2 & 40 & 4.5 & 1 \\
3 & & 3.5 & 2 \\
4 & & 3.5 & 2 \\
5 & & 3.5 & 2 \\
6 & & 2.5 & 3 \\
7 & & 4.5 & 3 \\
\hline 8 & 50 & 3.5 & 3 \\
9 & & 3.5 & 1 \\
10 & & 4.5 & 2 \\
11 & & 2.5 & 2 \\
\hline 12 & 30 & 2.5 & 2 \\
13 & & 4.5 & 2 \\
14 & & 3.5 & 1 \\
15 & & 3.5 & 3 \\
\hline
\end{tabular}


Table 10 Test order and conditions of the solvent extraction stage following the second subset of the modified experimental protocol.

\begin{tabular}{ccccc}
\hline Test No. & $\begin{array}{c}\text { Strippant Conc., } \\
\mathrm{H}_{2} \mathrm{SO}_{4}(\mathrm{~mol} / \mathrm{L})\end{array}$ & $\begin{array}{c}\text { Extractant Conc. } \\
(\mathrm{v} \% \mathrm{TBP})\end{array}$ & $\mathrm{pH}$ & $\begin{array}{c}\text { Organic/Aqueous } \\
\text { Ratio }\end{array}$ \\
\hline 1 & & 30 & 3.5 & 1 \\
2 & & 40 & 4.5 & 1 \\
3 & & 50 & 3.5 & 1 \\
4 & & 40 & 2.5 & 1 \\
5 & & 30 & 2.5 & 2 \\
6 & & 30 & 4.5 & 2 \\
7 & 1 & 30 & 3.5 & 3 \\
8 & & 50 & 3.5 & 3 \\
9 & & 40 & 3.5 & 2 \\
10 & & 50 & 2.5 & 2 \\
11 & & 40 & 3.5 & 2 \\
12 & & 40 & 3.5 & 2 \\
13 & & 40 & 4.5 & 3 \\
14 & & 40 & 2.5 & 3 \\
15 & & 50 & 4.5 & 2 \\
\hline 16 & & 40 & 4.5 & 2 \\
17 & & 50 & 3.5 & 2 \\
18 & & 40 & 3.5 & 3 \\
19 & 0.5 & 40 & 3.5 & 1 \\
20 & & 30 & 3.5 & 2 \\
21 & & 40 & 2.5 & 2 \\
\hline 22 & & 50 & 3.5 & 2 \\
23 & & 30 & 3.5 & 2 \\
24 & & 40 & 4.5 & 2 \\
25 & 2 & 40 & 3.5 & 1 \\
26 & & 40 & 3.5 & 3 \\
27 & & 40 & 2.5 & 2 \\
\hline
\end{tabular}

\subsubsection{Zeolite Adsorption Tests}

In this present study, zeolites, a group of crystalline alumina-silicates, were determined to be used for the adsorption tests due to their high chemical and radiation stability, unique physicochemical characteristics, high porosity, high sorption capacity, and many efficient applications to remove thorium and uranium from aqueous solutions by adsorption (Brindley and Bastovanov, 1982; Dyer and Jozefowicz, 1992; Misaelides et al., 1995; Kilincarslan and Akyil, 2005; Camacho et al., 2010). Zeolite's crystal lattice consists of two tetrahedral structures, $\mathrm{SiO}_{4}$ and $\mathrm{AlO}_{4}$ are arranged to share each oxygen atom. The crystal structure of a zeolite has a net negative charge due to the 
charge difference between aluminum and silicon, and it is balanced with the exchangeable cations (Metaxas et al., 2003). A representation of a zeolite structure is given in Figure 8. The adsorption tests were carried out in a batch system by mixing the known volume of feedstock solution with a suitable amount of sorbent for a period of time at a controlled temperature to achieve equilibrium. Then, the sorbent was separated by filtration using $0.45 \mu \mathrm{m}$ pore-sized filter paper, and the representative supernatant was subjected to thorium, uranium, rare earths, and major metal analyses using ICP-MS and ICP-OES.
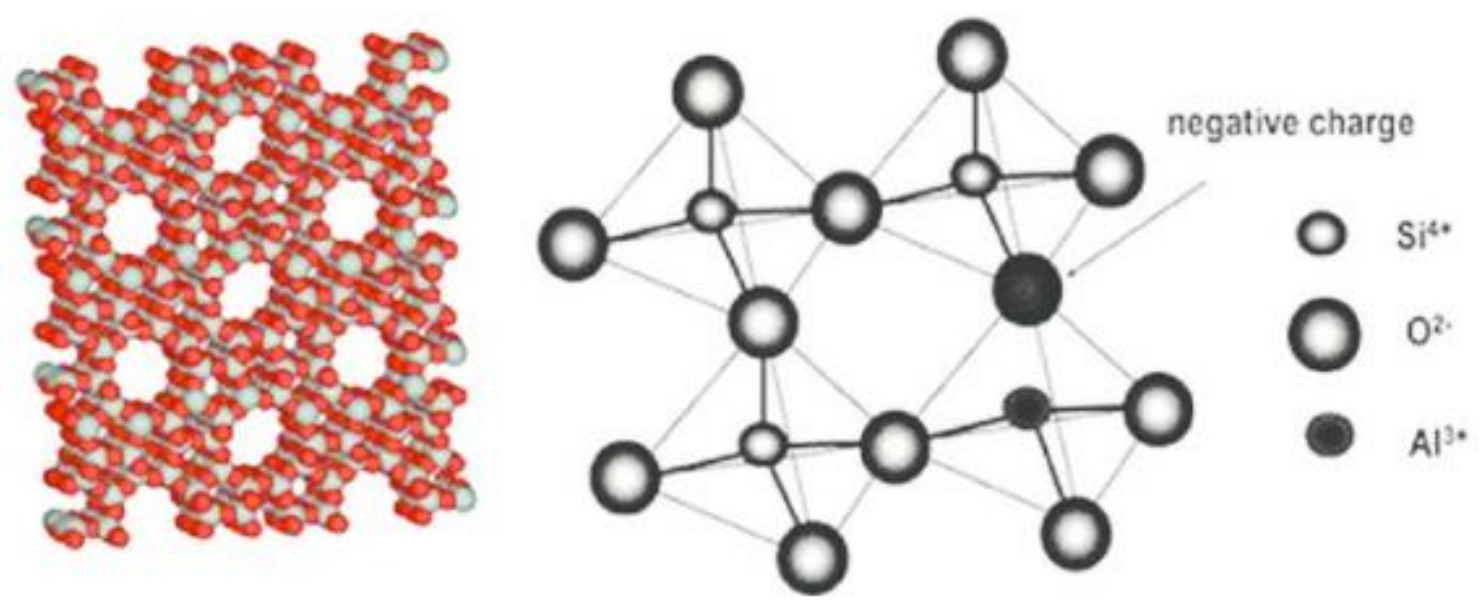

Figure 8 Structure of zeolite (after Zendelska et al., 2015).

\subsubsection{1. $\quad$ Experimental Design for Zeolite Adsorption Tests}

The effects of various operational parameters such as feed solution $\mathrm{pH}$, adsorbent particle size, zeolite amount, and contact time on the removal of thorium and uranium were investigated during zeolite adsorption tests. According to earlier studies, the solution $\mathrm{pH}$ was decided to vary between 2-4, (Kaygun and Akyil 2007; Baybas and Ulusoy, 2011; Bertetti et al., 2011; Fasfous and Dawoud, 2012; Li et al., 2018). A further increase in $\mathrm{pH}$ (above 4.5) typically leads to the precipitation of thorium, uranium, and rare earths which is why experiments are not usually performed at pH values higher than 4 (Kutahyali and Eral, 2010; Cheira et al., 2017). Additionally, when $\mathrm{pH}$ is less than 2, the decomposition of zeolite is noticed, which will result in ineffective and lower adsorption capacity (Baybas and Ulusoy, 2011). $2 \mathrm{~mol} / \mathrm{L}$ sodium hydroxide $(\mathrm{NaOH})$ was used to adjust the solution chemistry to achieve different $\mathrm{pH}$ values as needed. Following previously conducted studies, $1 \mathrm{~h}, 2 \mathrm{~h}$, and $3 \mathrm{~h}$ contact periods were determined for the tests (Kutahyali and Eral, 2010; Sani et al., 2015). Zeolite samples were used as received, without any pretreatment. An experimental design was generated with each parameter has three levels, and a total of 27 tests were performed following the operational conditions seen in Table 11. 
Table 11 Test order and conditions of zeolite adsorption tests.

\begin{tabular}{|c|c|c|c|c|}
\hline Test No. & Particle Size $(\mu \mathrm{m})$ & $\mathrm{pH}$ & Adsorbent Amount (g) & Contact Time (hr) \\
\hline 1 & 12 & 3 & 5.00 & 2 \\
\hline 2 & 1490 & 2 & 3.75 & 2 \\
\hline 3 & 200 & 4 & 3.75 & 1 \\
\hline 4 & 12 & 4 & 3.75 & 2 \\
\hline 5 & 1490 & 3 & 2.50 & 2 \\
\hline 6 & 200 & 3 & 3.75 & 2 \\
\hline 7 & 200 & 4 & 2.50 & 2 \\
\hline 8 & 200 & 3 & 5.00 & 3 \\
\hline 9 & 12 & 3 & 3.75 & 1 \\
\hline 10 & 200 & 3 & 2.50 & 3 \\
\hline 11 & 12 & 3 & 2.50 & 2 \\
\hline 12 & 1490 & 3 & 3.75 & 1 \\
\hline 13 & 1490 & 4 & 3.75 & 2 \\
\hline 14 & 200 & 3 & 3.75 & 2 \\
\hline 15 & 12 & 3 & 3.75 & 3 \\
\hline 16 & 200 & 2 & 3.75 & 3 \\
\hline 17 & 200 & 3 & 2.50 & 1 \\
\hline 18 & 200 & 2 & 5.00 & 2 \\
\hline 19 & 200 & 4 & 5.00 & 2 \\
\hline 20 & 200 & 4 & 3.75 & 3 \\
\hline 21 & 200 & 3 & 3.75 & 2 \\
\hline 22 & 1490 & 3 & 3.75 & 3 \\
\hline 23 & 12 & 2 & 3.75 & 2 \\
\hline 24 & 200 & 3 & 2.50 & 1 \\
\hline 25 & 1490 & 3 & 3.75 & 2 \\
\hline 26 & 200 & 2 & 3.75 & 2 \\
\hline 27 & 200 & 2 & 2.50 & 1 \\
\hline
\end{tabular}

Following the test results, the adsorption recovery of thorium, uranium, and rare earths were calculated using the equation below:

$$
\text { Adsorption Recovery }(\%)=\frac{C_{i-} C_{f}}{C_{i}} \times 100
$$

where $C_{i}$ and $C_{f}$ are the concentration $(\mathrm{mg} / \mathrm{L})$ of the ions of interest in the aqueous solution before and after adsorption, separately. The results were also examined in terms of adsorption capacity and distribution coefficient according to the following equations:

$$
\text { Sorption Capacity }(q)=\left(C_{i-} C_{f}\right) \times \frac{V}{m}
$$




$$
\text { Distribution Coefficient }\left(K_{d}\right)=\frac{C_{i-} C_{f}}{C_{i}} \times \frac{V}{m}
$$

where $\mathrm{V}$ is the volume of the solution $(\mathrm{L}), \mathrm{m}$ is the weight of zeolite $(\mathrm{g})$ used, and $\mathrm{C}_{\mathrm{e}}$ is the ion concentration $(\mathrm{mg} / \mathrm{L})$ when adsorption equilibrium is reached. The distribution coefficient, which defines the ratio of ion of interest in the sorbent and the solution at equilibrium, is a direct indicator of the selectivity of the adsorbent towards the ions of interest (Metaxas et al., 2003).

\subsection{Sample Characterization}

\subsubsection{Characterization of Solids Generated from Selective Precipitation}

The dry solids produced from selective precipitation tests were subjected to X-ray Diffraction (XRD) and Scanning Electron Microscopy-Energy Dispersive X-ray Spectroscopy (SEM-EDX) analyses. XRD and SEM-EDX analytical techniques were applied to detect the mineralogy, morphology, and elemental compositions of the solid samples. Powdered samples were mounted in cavity mounts, and XRD patterns were collected using a Malvern PANalytical X'Pert Pro diffractometer equipped with copper radiation and an $\mathrm{X}^{\prime}$ Celerator parallel plate detector. The samples were scanned at $45 \mathrm{kV}, 40 \mathrm{~mA}$ over a range of $10-85^{\circ}$ two-theta in continuous mode. Evaluation and refinement of the diffraction pattern were carried out using the HighScore software.

The Hitachi S4700 Scanning Electron Microscope was used for the SEM-EDX analysis. Prior to analysis, the sample was subjected to sputtering using Denton Desk V Sputter and Carbon Coater to prevent charging and improve the quality of the image. During the analysis, accelerating voltage and working distance were kept at $5 \mathrm{kV}$ and $12 \mathrm{~mm}$, respectively. The XRD instrument and the SEM and sample preparation instruments used during the characterization studies are seen in Figure 61 and Figure 62 in Appendix.

\subsubsection{Characterization of Zeolite Samples}

A detailed characterization study was performed for the zeolite samples both before and after adsorption. First, the particle size analysis of each zeolite sample was performed using CILAS 1190 particle size analyzer, as shown in Figure 63. Following that, samples' mineralogical compositions were determined using the aforementioned PanAnalytical X'Pert Pro X-ray diffractometer. Samples were scanned at $45 \mathrm{kV}$ and $40 \mathrm{~mA}$ over a $10-90^{\circ}$ two-theta angle range in a continuous mode. As described previously, the evaluation and refinement of diffraction patterns were carried out using the HighScore software. The chemical composition of the samples was identified using the Olympus Vanta handheld X-ray fluorescence (XRF) analyzer (Figure 64). The same SEM-EDX instrument was utilized to analyze the samples' morphology. The accelerating voltage was varied between 5 and $15 \mathrm{kV}$, and the working distance was maintained at $12 \mathrm{~mm}$ during the SEM-EDX analysis. Similar to the other solid samples, before SEM-EDX analysis, zeolite samples were subjected to sputtering for 140 seconds using the Denton Desk V Sputter and Carbon Coater to enhance the image quality. SEM images taken under the lowest magnification were also analyzed with ImageJ image processor for additional particle size measurement. Lastly, 
the three zeolite feed samples were subjected to the Brunauer-Emmett-Teller (BET) nitrogen adsorption tests using the Micrometrics BET instrument (Figure 65) equipped with the ASAP 2020 Version 4.02 to identify the pore volume, pore size, and specific surface area. 


\section{CHAPTER 4}

\section{SELECTIVE PRECIPITATION AND SOLVENT EXTRACTION STUDIES}

\subsection{Preliminary Selective Precipitation Tests}

The initial precipitation data resulting from the addition of the three alkaline salts is shown in Figure 9. Test results indicate no significant difference observed between various alkaline salts with respect to the process separation efficiency. Therefore, the precipitation data obtained from all three $\mathrm{pH}$ adjustments were grouped and shown in Figure 9, and $\mathrm{NaOH}$ was chosen for subsequent experimental testing.

It can be seen from Figure 9 that the first precipitation was observed in a pH range of 4 to 5 . Under this condition, over $90 \%$ of thorium precipitated out while a small portion of uranium (i.e., $28.7 \%$ ) and REEs (i.e., 16.3\%) co-precipitated with thorium. As indicated in the study of Zhu et al. (2015), thorium's precipitation $\mathrm{pH}$ range is $2.5-5.5$, which is consistent with the findings presented in this work. Thorium initially existed in the solution as $\mathrm{Th}^{4+}$ followed by being converted to thorium hydroxide with an increase in the solution $\mathrm{pH}$. However, thorium hydroxide is unstable by nature and eventually transforms into thorium dioxide (Brookins, 1988). A further increase in the $\mathrm{pH}$ to a value of 6.2 resulted in higher thorium and uranium precipitation and a significant amount of rare earth co-precipitation, which was approximately 34\%. The observed phenomena can be attributed to the fact that the precipitation $\mathrm{pH}$ range of uranium (i.e., 5.5-7) and rare earths (i.e., 6.8-8.0) overlaps to some degree which leads to the co-precipitation of the two (Zhu et al., 2015). Detailed results for individual rare earth elements, thorium and uranium precipitation, and their concentrations in the filtrates at various $\mathrm{pH}$ values are given in the Appendix.

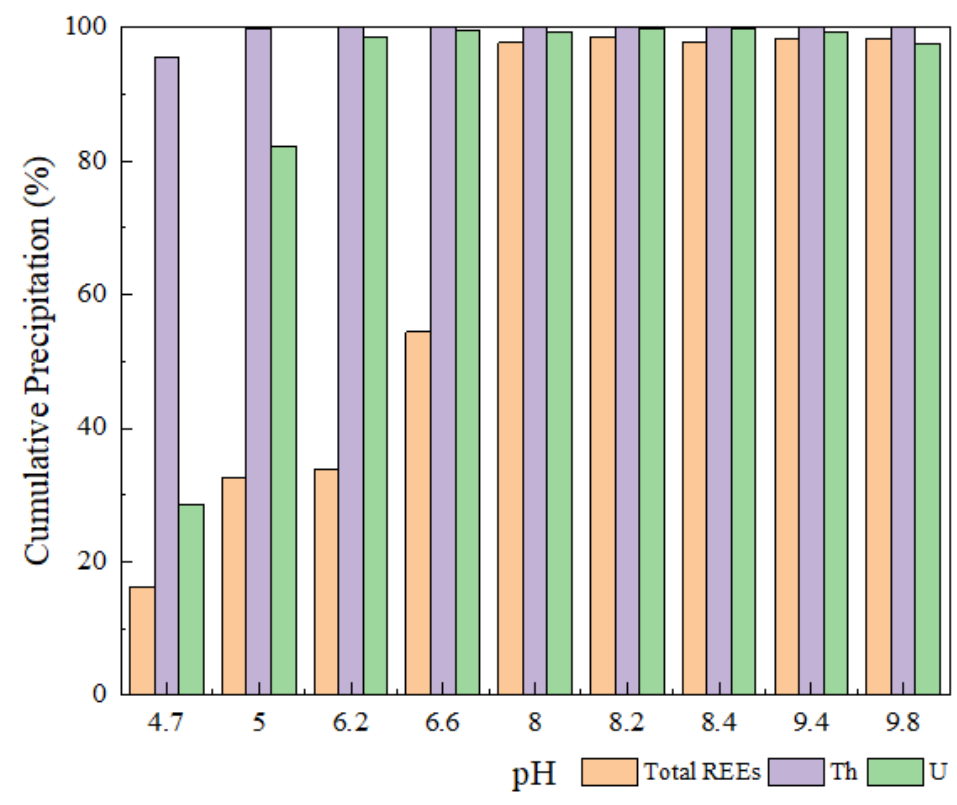

Figure 9 Cumulative precipitation of total rare earths, thorium, and uranium at various $\mathrm{pH}$ values. 
It is known that most rare earths and uranium existed in the synthetic solution as REEs ${ }^{3+}$ and $\mathrm{U}^{6+}$ along with $\mathrm{Th}^{4+}$ since standard ICP solutions had been used for the feedstock preparation. Chemical reactions governing the precipitation behavior of $\mathrm{REEs}^{3+}, \mathrm{Th}^{4+}$, and $\mathrm{U}^{6+}$ are given below (Silva et al., 2018). As such, rare earth hydroxide, thorium dioxide, and uranyl hydroxide are formed at their respective precipitation $\mathrm{pH}$ regions.

$$
\begin{aligned}
& \operatorname{REEs}_{(a q)}^{3+}+3 \mathrm{OH}_{(a q)}^{-}=\operatorname{REES}(\mathrm{OH})_{3(s)} \\
& \mathrm{Th}_{(a q)}^{4+}+4 \mathrm{OH}_{(a q)}^{-}=\mathrm{ThO}_{2(\mathrm{~s})}+2 \mathrm{H}_{2} \mathrm{O} \\
& U \mathrm{O}_{2(a q)}^{2+}+2 \mathrm{OH}_{(a q)}^{-}=\mathrm{UO}_{2}(\mathrm{OH})_{2(s)}
\end{aligned}
$$

The precipitation behavior of total, heavy, light, critical, and uncritical rare earth elements are further examined and presented in Figure 10. As seen, the precipitation of heavy/light and critical/uncritical REEs was minimal at a $\mathrm{pH}$ value of 4.7. It also suggests that the precipitation of heavy rare earths was relatively more significant in a $\mathrm{pH}$ range of 5 to 8 compared to light rare earths. Similar trend was also observed for the precipitation of critical rare earth elements in the same $\mathrm{pH}$ region, which is expected since the majority of critical elements are also belong to the heavy rare earth group. Approximately $100 \%$ rare earth precipitation was achieved when the $\mathrm{pH}$ was above 8 . The same trend and findings were observed in the studies carried out by Ponou et al. (2016) and Honaker et al. (2018), indicating that rare earths precipitation becomes significant when the $\mathrm{pH}$ value is beyond 5, and nearly $90 \%$ of rare earths precipitation occurs in a $\mathrm{pH}$ range of 5 to 10. Therefore, it can be concluded that selective precipitation is effective for the removal of thorium from rare earths and uranium at a $\mathrm{pH}$ value of less than 5. However, the fact that a further increase in the $\mathrm{pH}$ of the leachate led to the co-precipitation of uranium and rare earths indicates an alternative technique ought to be evaluated to fully separate uranium from rare earths. 


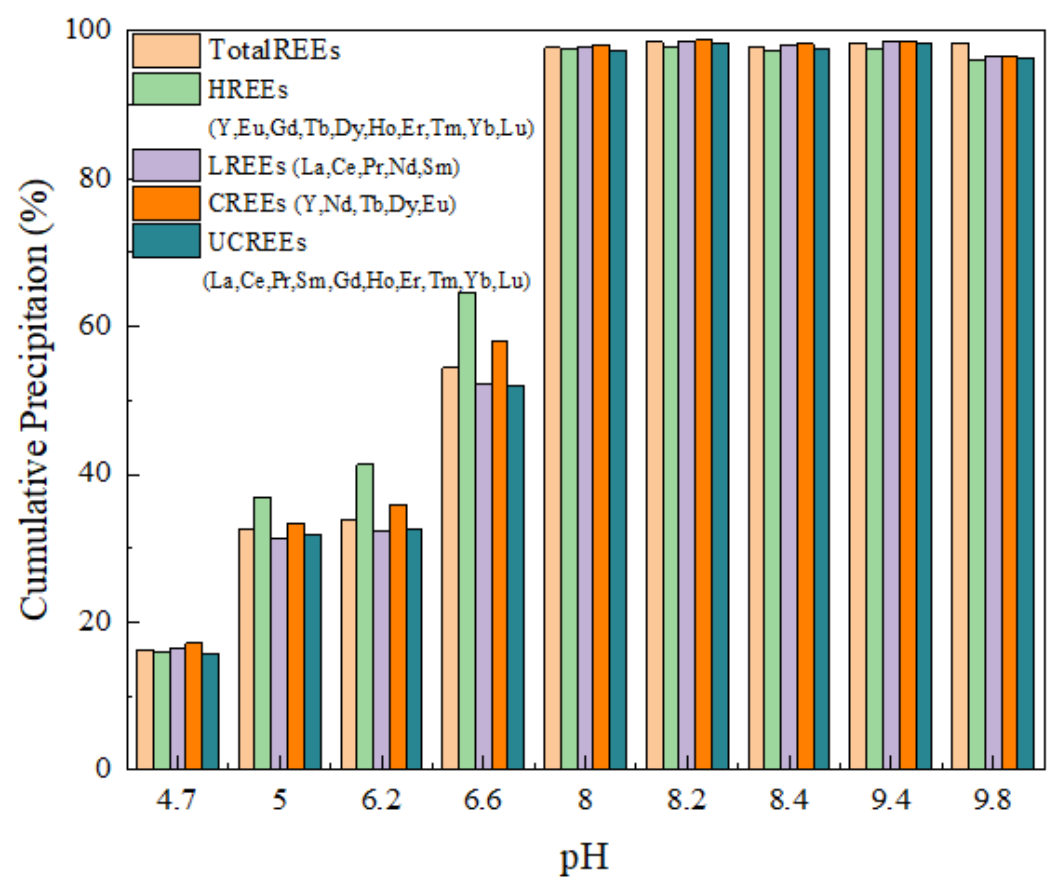

Figure 10 Cumulative precipitation of total, heavy, light, critical, and uncritical rare earths at various $\mathrm{pH}$ values.

\subsection{Preliminary Solvent Extraction Tests}

Rare earth-enriched product, organic, and effluent streams were produced at the end of each set of solvent extraction tests (Figure 6). The content of individual rare earth, thorium, and uranium in the rare earth product stream for both the one-stage and two-stage process is provided in Table 12.

The results shown in Figure 11 indicate that approximately 97\% of rare earths and 94\% of thorium were recovered into the circuit product stream of the two-stage process. Under the same operational conditions, $43 \%$ of uranium was recovered into the circuit product stream. For the onestage process, the recovery of rare earth elements, thorium, and uranium into the product stream was $90 \%, 93 \%$, and $51 \%$ separately. Regardless of the number of stages conducted for the solvent extraction process (one-stage vs. two stages), the recovery of thorium did not fluctuate much; however, a noticeable difference was observed for the recovery of rare earths and uranium. When the number of operating stages for extraction, scrubbing, and stripping was increased from one to two, the recovery of uranium was reduced from an initial value of $51 \%$ to $43 \%$, while the rare earth element recovery was increased from around $90 \%$ to $97 \%$. Therefore, the efficiency of the multistage solvent extraction process was indicated as opposed to the single-stage process with respect to the exaction of REEs and the removal of uranium. However, no significant separation of thorium was observed compared to uranium (Table 12), which may be explained by their distinct extraction behaviors in hydrochloric acid. Qi (2018) studied the extraction mechanism of thorium, uranium, and rare earths in the TBP-HCl system and stated that the extraction order is $\mathrm{UO}_{2}{ }^{2+}>\mathrm{Th}^{4+}>\mathrm{REE}^{3+}$. When present with the three ion groups, TBP forms complexes with the ions in the solution 
according to their distinctive capabilities to bind with the extractant (Rand et al., 2008). The complexation reactions occurring between TBP and the three ion groups (i.e., U, Th, and REE) are given in Eqs. (14)-(16). Similarly, Nasab (2014) observed that the extraction of uranium is more feasible compared to thorium in a chloride medium, which is attributed to the species formed between uranium and hydrochloride that TBP readily extracts through forming organic soluble complexes. Another reason is that thorium purification by solvent extraction in nitric acid media is more favorable (Al-Areqi et al., 2014). Nitrate is a preferred medium for enhanced extraction of thorium due to an associated increase in the hydration energy of the anions (Nasab, 2014; Lu et al., 2016). In addition, $2 \mathrm{~mol} / \mathrm{L}$ nitric acid $\left(\mathrm{HNO}_{3}\right)$ solution was used as the scrubbing reagent to remove entrained rare earth elements and thorium from the U-laden organic phase following the reactions given in Eqs. (17) and (18). Afterward, DI water and $2 \mathrm{~mol} / \mathrm{L} \mathrm{HNO}_{3}$ were applied as the stripping reagent for thorium and uranium, separately, and the corresponding equations governing the complexation reactions are shown in Eqs. (19) and (20).

The recovery of individual rare earths into the product stream shown in Figure 12 indicates that the majority of the elements achieved a recovery above $90 \%$. In comparison, a relatively lower recovery was noticed for scandium $(<90 \%)$. The same phenomenon for scandium was also observed in Qi's (2018) study saying that in a high hydrochloric acid medium, the separation factor of scandium is much higher, resulting in transferring scandium into the organic phase. Moreover, Figure 13 suggests that no substantial difference was observed for the recovery of HREEs, LREEs, CREEs, and UCREEs for each of the two sets of solvent extraction tests. An improved recovery was obtained for the two-stage process. Detailed results are given in Table 25, Table 26, and Table 27 in the Appendix.
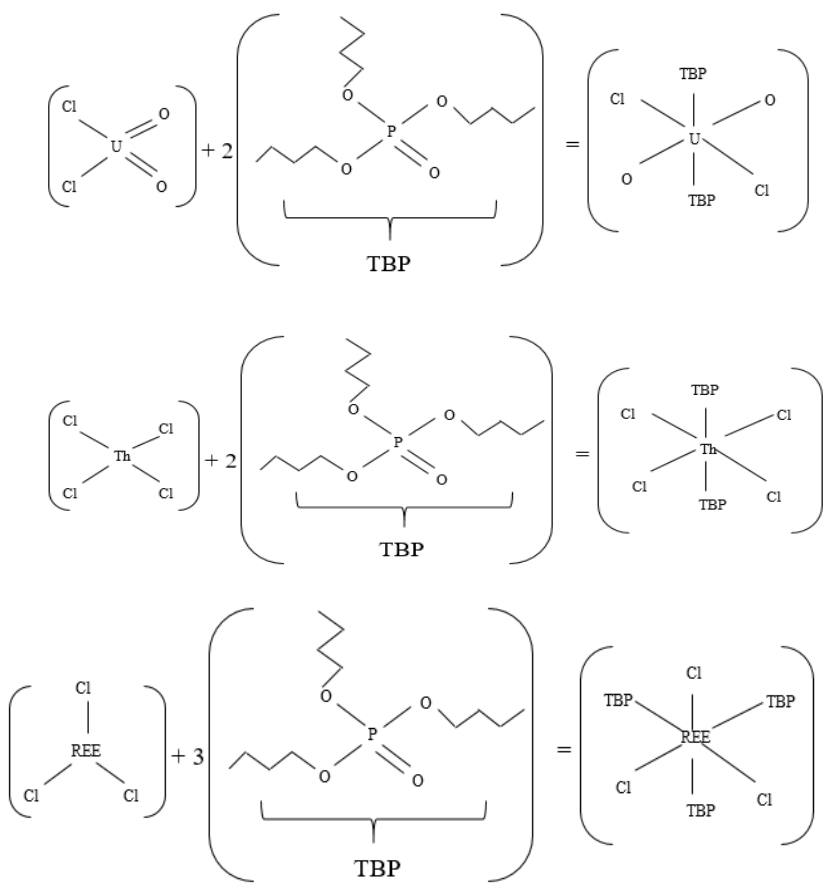


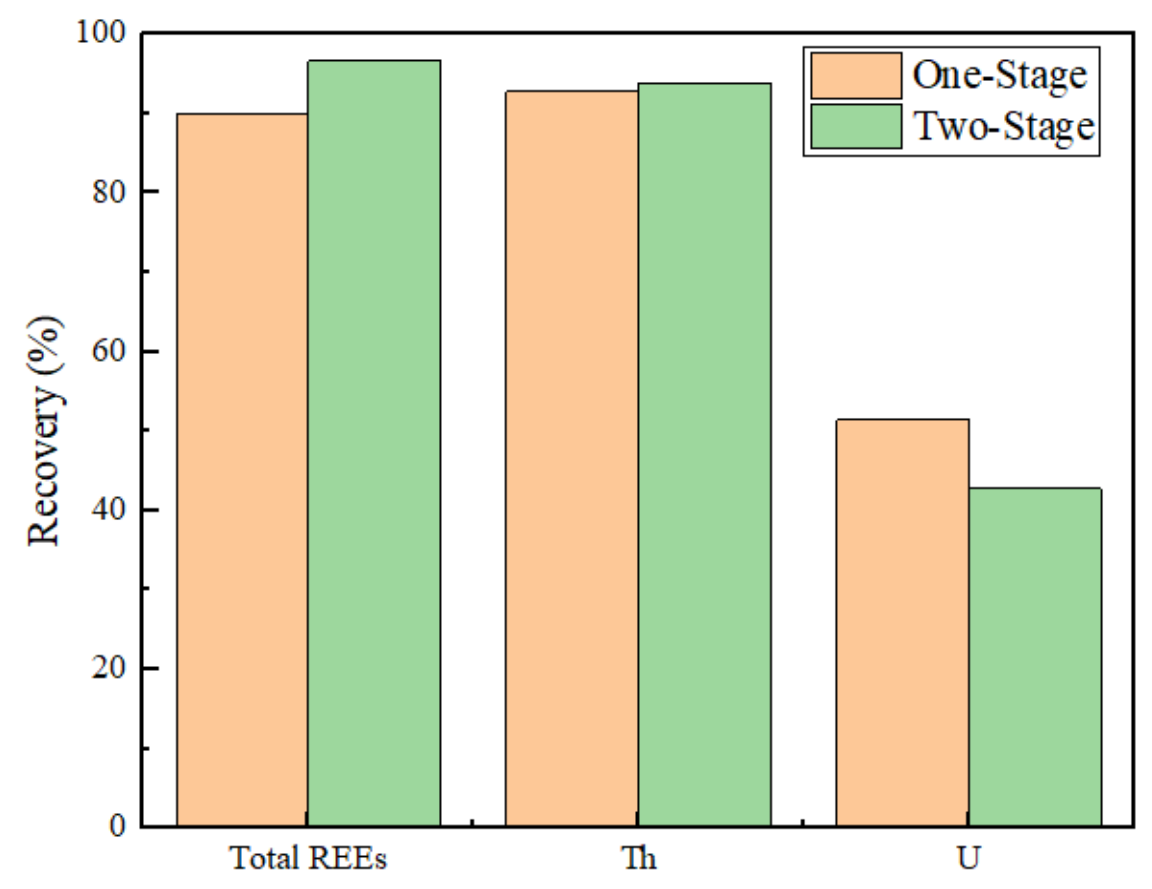

Figure 11 Recovery of total rare earths, thorium, and uranium into the REE product stream of both the one-stage and two-stage solvent extraction process.

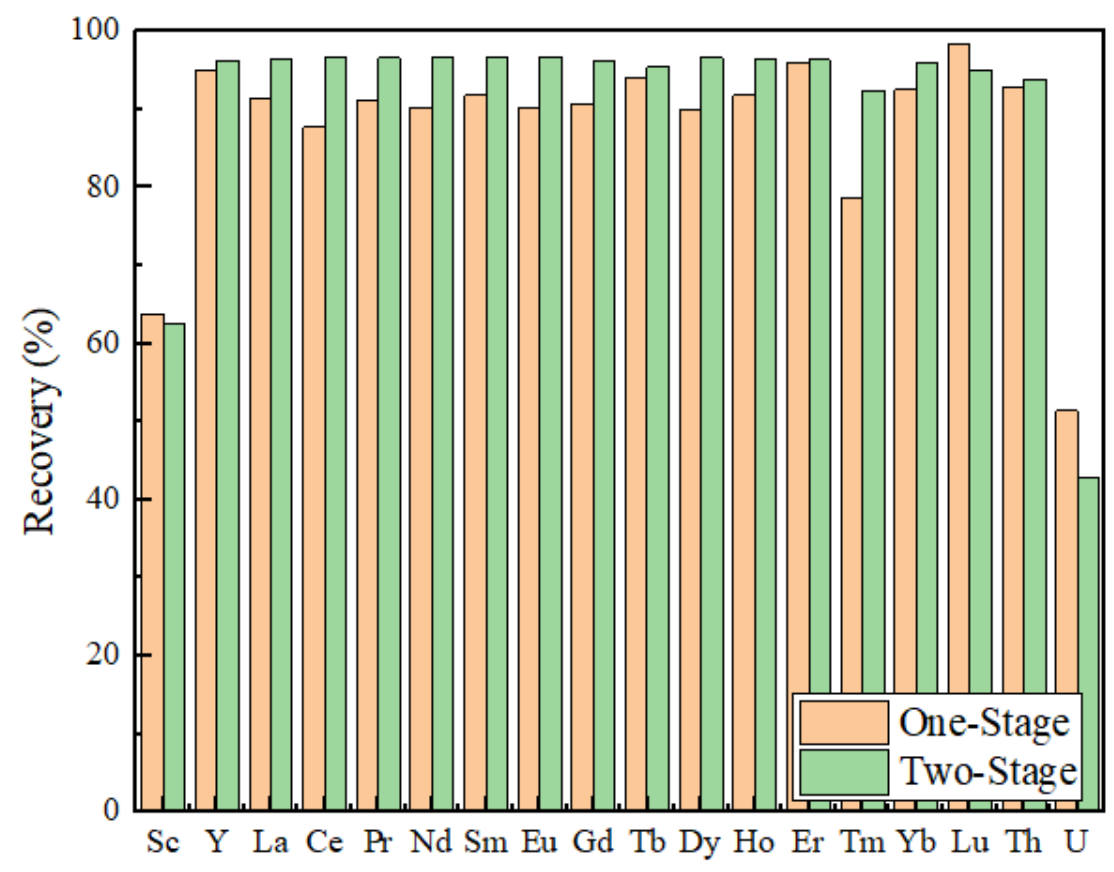

Figure 12 Recovery of individual rare earth, thorium, and uranium into the REE product stream of both the one-stage and two-stage solvent extraction process. 
Table 12 Individual rare earth, thorium, and uranium concentrations (ppm) in the rare earth product stream obtained from both the one-stage and two-stage solvent extraction process.

\begin{tabular}{ccccccccccccccccccc}
\hline Process & $\mathrm{Sc}$ & $\mathrm{Y}$ & $\mathrm{La}$ & $\mathrm{Ce}$ & $\mathrm{Pr}$ & $\mathrm{Nd}$ & $\mathrm{Sm}$ & $\mathrm{Eu}$ & $\mathrm{Gd}$ & $\mathrm{Tb}$ & $\mathrm{Dy}$ & $\mathrm{Ho}$ & $\mathrm{Er}$ & $\mathrm{Tm}$ & $\mathrm{Yb}$ & $\mathrm{Lu}$ & $\mathrm{Th}$ & $\mathrm{U}$ \\
\hline One-Stage & 0.00 & 1.49 & 0.85 & 6.17 & 0.84 & 3.95 & 1.18 & 0.20 & 0.88 & 0.04 & 0.36 & 0.04 & 0.16 & 0.00 & 0.06 & 0.01 & 0.21 & 0.20 \\
Two-Stage & 0.00 & 1.62 & 0.94 & 7.16 & 0.96 & 4.42 & 1.34 & 0.24 & 1.04 & 0.05 & 0.43 & 0.05 & 0.19 & 0.00 & 0.08 & 0.01 & 0.26 & 0.09 \\
\hline
\end{tabular}

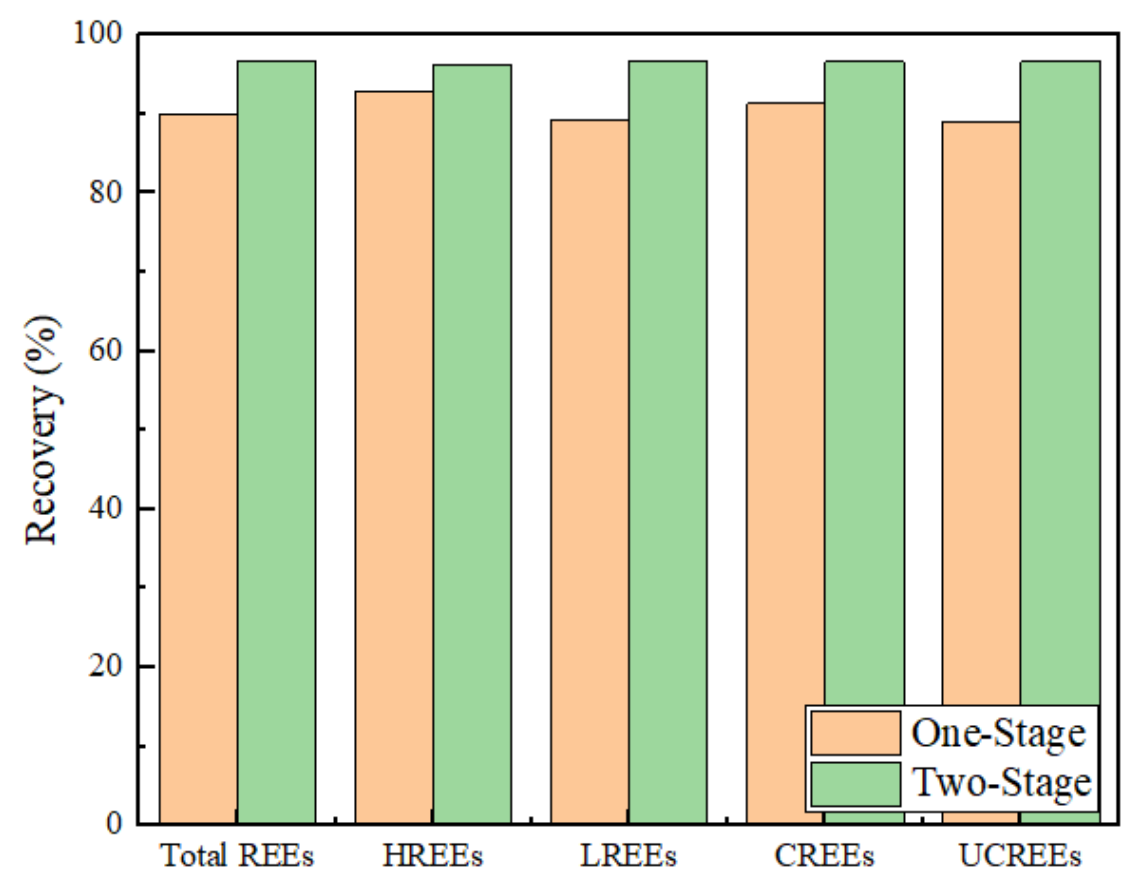

Figure 13 Recovery of total, heavy, light, critical and uncritical REEs from both the one-stage and two-stage solvent extraction process. 

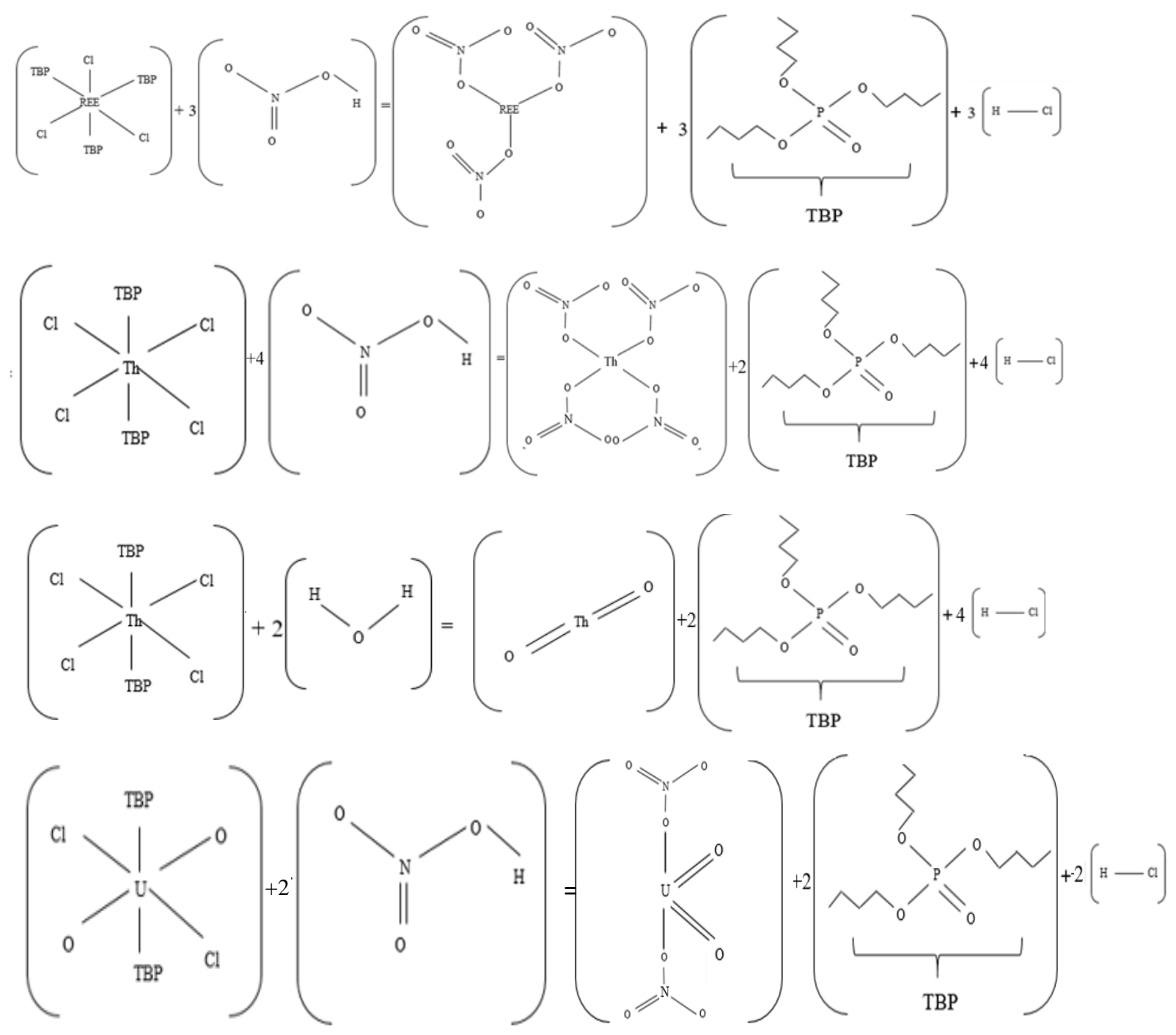


\subsection{Modified Experimental Protocol}

\subsubsection{One-Stage Precipitation}

As seen from the results presented earlier, selective precipitation successfully precipitated out thorium, while solvent extraction was noticeably more effective in removing uranium from rare earths. Therefore, a modified experimental protocol consisting of one-stage precipitation and twostage solvent extraction was developed, the procedure of which was strictly followed for improved separation of the radioactive elements from rare earths.

Individual REE content and the concentration of thorium and uranium of the filtrate obtained at the precipitation $\mathrm{pH}$ of 4.85 are shown in Table 13. All the data reported are the average of three replicate selective precipitation tests. It can be seen from Table 13 that thorium present in the feedstock solution all precipitated out as solids, and no content was detected in the remaining solution, which further confirms the findings previously discussed in selective precipitation. Besides, the amount of REEs, uranium, and major metals along with thorium precipitated at a $\mathrm{pH}$ of 4.85 was shown in Figure 14.

As seen, $100 \%$ of thorium precipitated out, while approximately $19.32 \%$ of rare earth elements and $47.98 \%$ of uranium co-precipitated at the same $\mathrm{pH}$ value. A similar result for uranium precipitation was also obtained by Tomazic et al. (1962). Furthermore, nearly $25 \%$ of major metals, including aluminum, calcium, iron, and magnesium, were transformed into solid forms under identical conditions. As for major metals, approximately $65.58 \%$ of iron precipitated, followed by around $38.51 \%$ of aluminum, $17.12 \%$ of calcium, and $14.17 \%$ of magnesium. The findings presented here are consistent with prior studies (Ladeira and Goncaves, 2007; Xie et al., 2014; Balintova and Petrilakova, 2011; Paul and Campbell, 2011). It is known that the precipitation of iron starts at a $\mathrm{pH}$ value of 3.5 , and $\mathrm{Fe}(\mathrm{OH})_{3}$ is formed as a result of iron oxidization (Seo et al., 2017). In the present study, iron had existed in the solution as ferric iron, which was ready to precipitate with an increase in the $\mathrm{pH}$, as seen in Eq. (21). On the other hand, the precipitation of aluminum typically initiates around a $\mathrm{pH}$ value of 5 (Balintova and Petrilakova, 2011), which results in the formation of $\mathrm{Al}(\mathrm{OH})_{3}$ in the presence of $\mathrm{OH}-$ ions following Eq. (22). Therefore, a substantially higher amount of iron precipitated out as opposed to aluminum, which also agrees well with the study carried out by Seo et al. (2017). High $\mathrm{Fe}(\mathrm{OH})_{3}$ precipitation may partially contribute to the co-precipitation of rare earths due to its capability to absorb particles onto the iron crystals' lattice sites (Silva et al., 2018).

Supplemental information can be derived by further analyzing the rare earth precipitation data, as shown in Figure 15. It suggests again that heavy REEs tend to preferentially precipitate out compared to light REEs, which leads to a precipitation difference of approximately 4.5 percent. However, this difference is less than one standard deviation, which may not be statistically significant. 
Table 13 Individual rare earth, thorium, and uranium concentration (ppm) of the filtrate obtained at the $\mathrm{pH}$ value of 4.85 .

\begin{tabular}{|c|c|c|c|c|c|c|c|c|c|c|c|c|c|c|c|c|c|}
\hline $\mathrm{Sc}$ & $Y$ & $\mathrm{La}$ & $\mathrm{Ce}$ & $\mathrm{Pr}$ & $\mathrm{Nd}$ & $\mathrm{Sm}$ & $\mathrm{Eu}$ & $\mathrm{Gd}$ & $\mathrm{Tb}$ & Dy & Ho & $\mathrm{Er}$ & $\mathrm{Tm}$ & $\mathrm{Yb}$ & $\mathrm{Lu}$ & Th & $\mathrm{U}$ \\
\hline 0 & 0.50 & 0.33 & 2.55 & 0.25 & 1.49 & 0.67 & 0.08 & 0.29 & 0.03 & 0.13 & 0.02 & 0.06 & 0.00 & 0.02 & 0.00 & 0.00 & 0.13 \\
\hline
\end{tabular}

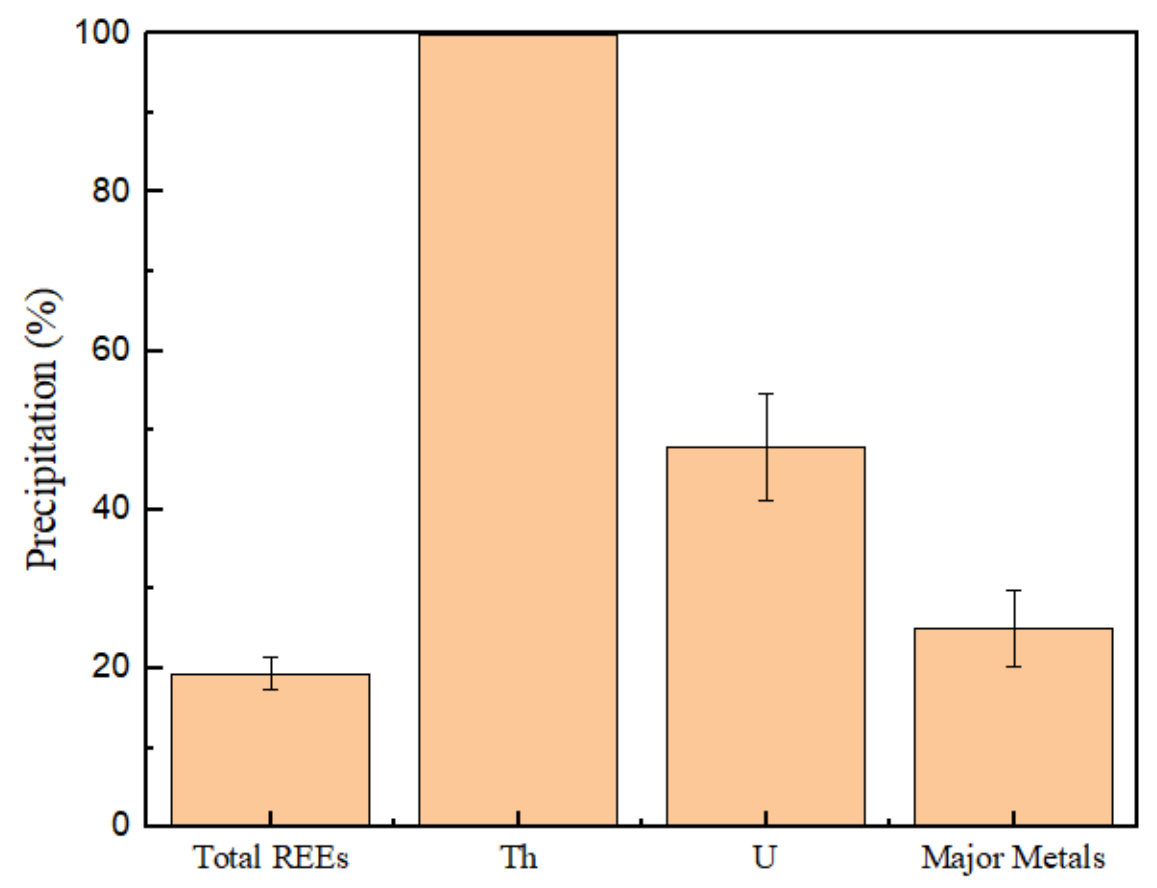

Figure 14 Average precipitation of total REEs, Th, U, and major metals at the $\mathrm{pH}$ value of 4.85 . Error bars one standard deviation of three replicate tests. 


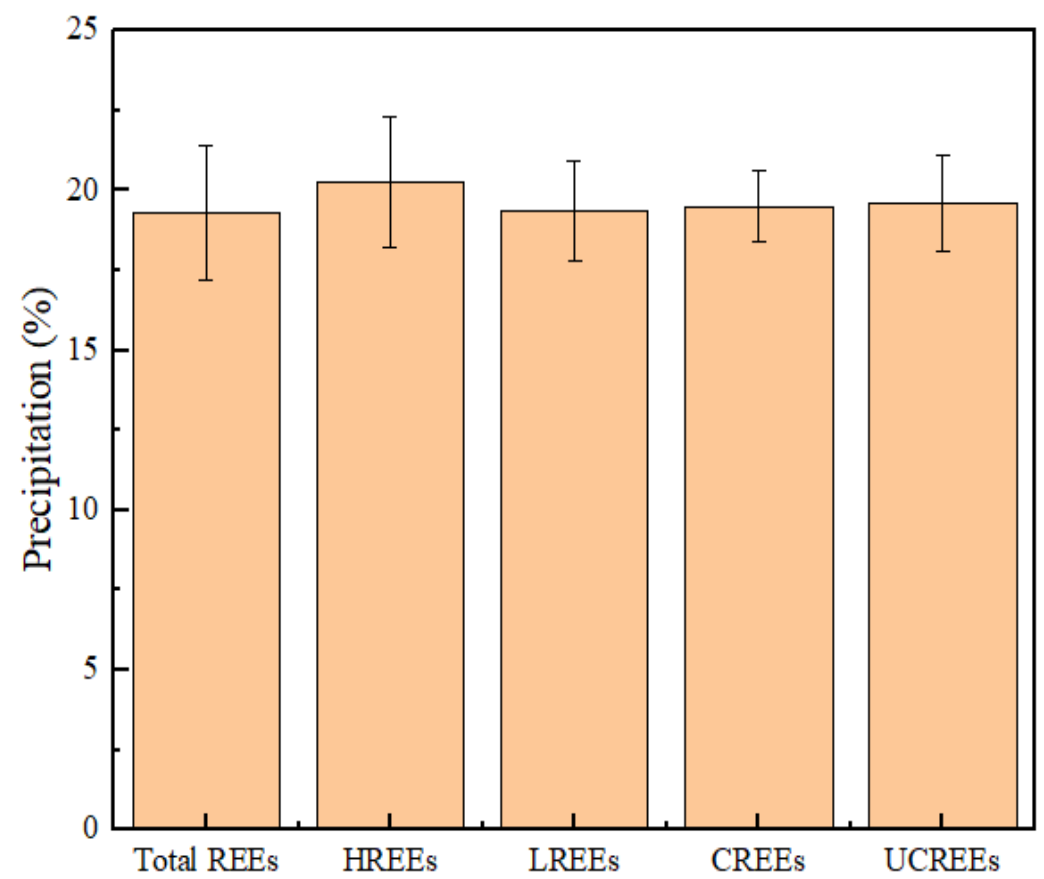

Figure 15 The average precipitation of total, heavy, light, critical, and uncritical rare earths at the $\mathrm{pH}$ value of 4.85. Error bars represent one standard deviation of three replicate tests.

$$
\begin{aligned}
& F e_{(a q)}^{3+}+3 O H_{(a q)}^{-}=F e(O H)_{3(s)} \\
& A l_{(a q)}^{3+}+3 O H_{(a q)}^{-}=A l(O H)_{3(s)}
\end{aligned}
$$

\subsubsection{Characterization of Solids Generated from Selective}

The XRD pattern of the solid sample obtained from the one-stage precipitation is shown in Figure 16. It can be seen that precipitated solids predominantly consisted of halite $(\mathrm{NaCl})$, which is attributed to the existence of sodium hydroxide and hydrochloric acid that were used to adjust the solution $\mathrm{pH}$ values according to various needs. In addition to halite, a relatively small amount of iron oxide was also detected, which corroborates the prior finding that approximately $66 \%$ of iron precipitated out from the feedstock solution and was transformed into iron oxide. Elemental identification of the solid sample was provided by SEM-EDX analysis as opposed to the qualitative analysis provided by XRD. As seen in Figure 17, the highest intensity peaks were observed for the elements of sodium $(\mathrm{Na})$, oxygen $(\mathrm{O})$, and chloride $(\mathrm{Cl})$, which is consistent with the findings obtained from XRD analysis. In addition to the three elements, a portion of rare earths and thorium were also detected in the EDX spectrum; however, the intensities are considerably low compared to $\mathrm{Na}, \mathrm{O}$, and $\mathrm{Cl}$. The carbon peak seen in the EDX spectrum is due to the carbon tape substrate used during sample preparation. Moreover, SEM images presented in Figure 18 show the rough surfaces of the solid particles. The microscopic pictures observed under high magnifications further indicate that tiny nuclei in nanoscale initially precipitated out and deposited on top of the 
particles, which acted like the nucleating agents and led to the subsequent growth of the particles in the form of roughly spherical crystallites.

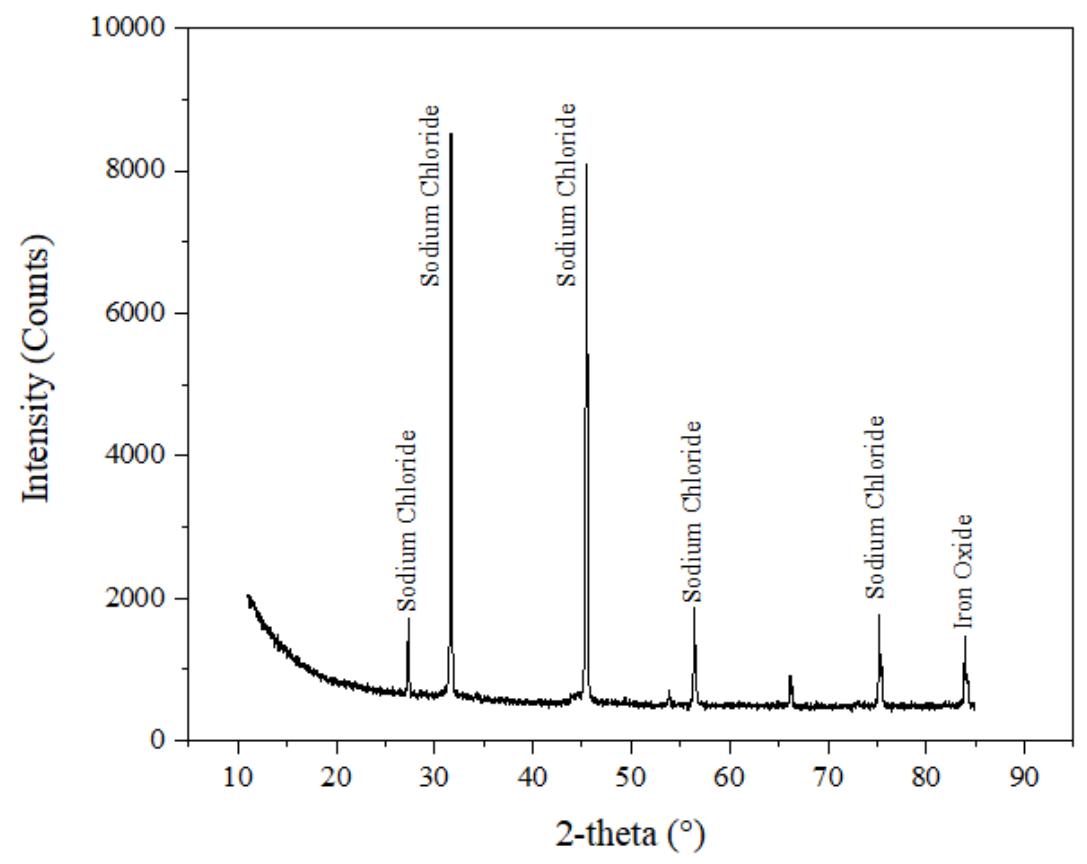

Figure 16 XRD pattern of the solids samples obtained from selective precipitation tests at a $\mathrm{pH}$ value of 4.85 .

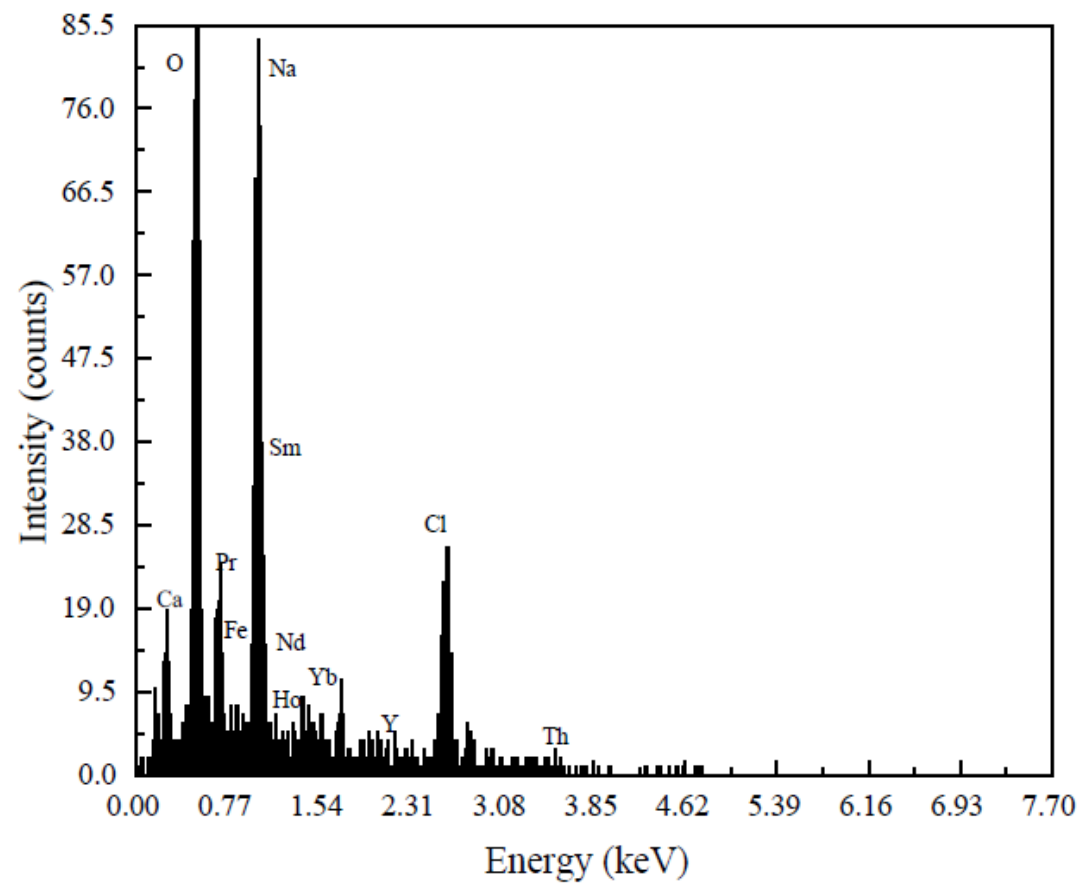

Figure 17 Elemental identification of the solids samples obtained from selective precipitation at a $\mathrm{pH}$ of 4.85 using SEM-EDX analysis. 

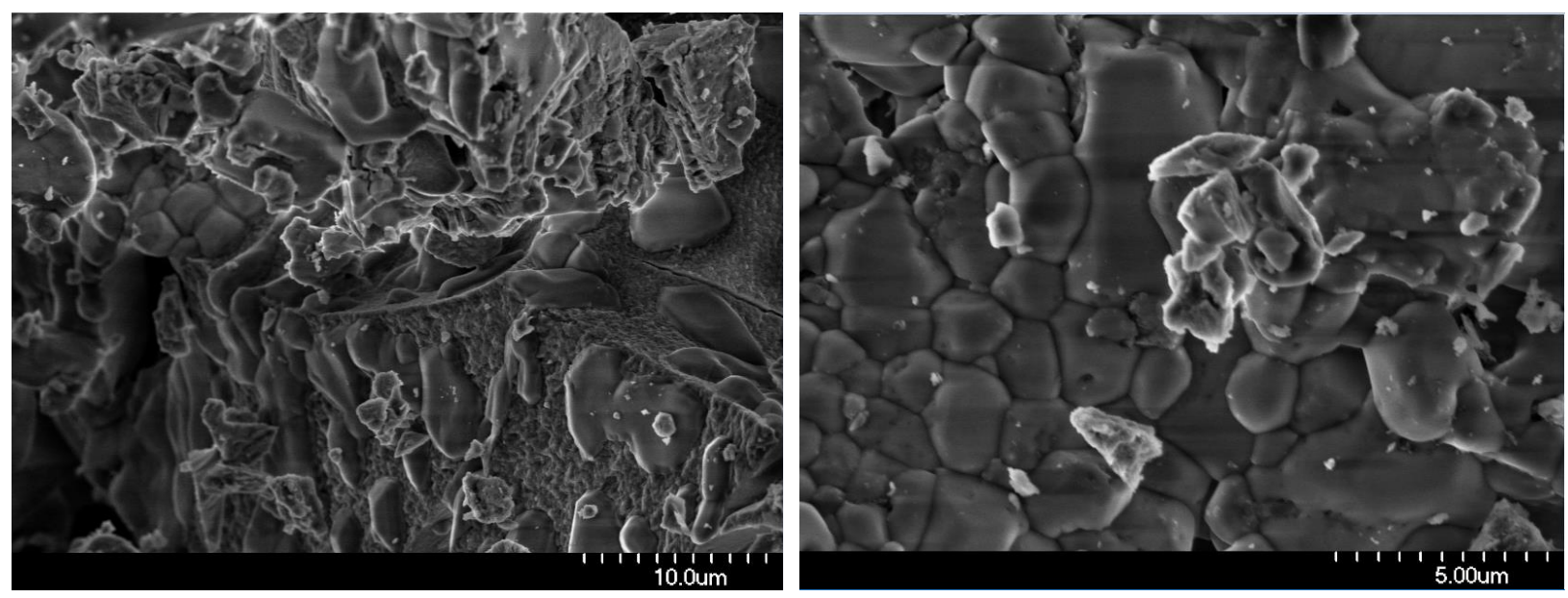

Figure 18 SEM images of precipitated solids (at a pH of 4.85) observed under various magnifications.

\subsubsection{Equilibrium and Thermodynamic Evaluation of Selective}

The predominance and stabilities of ionic and non-ionic species in aqueous solutions are critical to understanding the concepts of dissolution, leaching, and selective precipitation. This information can be displayed graphically using speciation (species) distribution diagrams and Eh$\mathrm{pH}$ (Pourbaix) diagrams. They are also helpful to identify major species existing at a particular $\mathrm{pH}$ value and provide the ability to predict the change in composition when there is a change in condition. Species distribution diagrams were constructed for selected rare earth elements, thorium, uranium, iron, and aluminum using the OLI Studio software based on their initial concentrations in the synthetic solution. It was reported that the existence of various species is notably pH-dependent (Li et al., 2012; Liu et al., 2015; Li et al., 2018) because pH value controls the ion activity (Monhemius, 1977; Hayes et al., 2002).

As seen in Figure 19, under acidic conditions, aqueous ions of $\mathrm{Th}^{4+}$ and $\mathrm{ThCl}^{3+}$ are seen. The concentration of $\mathrm{Th}^{4+}$ starts to decrease around a $\mathrm{pH}$ value of 3 , which is due to the $\mathrm{OH}^{-}$ions liberated from $\mathrm{NaOH}$ that initiates thorium's precipitation by forming hydroxide complexes. Likewise, the concentration of $\mathrm{ThCl}^{3+}$ starts to decrease at $\mathrm{pH}$ 3. Initially, $\mathrm{ThCl}^{3+}$ is formed due to a reaction between $\mathrm{Cl}^{-}$and $\mathrm{Th}^{4+}$ ions in the solution. However, when an alkaline is introduced to the solution, the reaction is reversed. It releases $\mathrm{Cl}^{-}$ions back into the solution, and free $\mathrm{Th}^{4+}$ ions react with $\mathrm{OH}^{-}$-forming thorium precipitates. Meanwhile, a substantial increase of thorium hydroxide $\left(\mathrm{Th}(\mathrm{OH})_{4}\right)$ is seen with an elevation in the solution $\mathrm{pH}$. In nature, $\mathrm{Th}(\mathrm{OH})_{4}$ may precipitate first; however, due to the thorium hydroxide's unstable nature, it slowly transforms into thorium dioxide $\left(\mathrm{ThO}_{2}\right)$ (Brookins 1988). It is stable as thorium dioxide $\left(\mathrm{ThO}_{2}\right)$ even in acidic conditions. The concentration of $\mathrm{Th}^{4+}$ and $\mathrm{Th}(\mathrm{OH})_{4}$ reaches the same level at a $\mathrm{pH}$ value of 5, for which the precipitation of thorium is assumed to be completed (Zhu et al., 2015). A further increase in the $\mathrm{pH}$ does not change the concentrations of $\mathrm{Th}^{4+}$ and $\mathrm{Th}(\mathrm{OH})_{4}$. The conclusions reached in this study are well correlated with the literature (Felmy et al., 1991). Thermodynamic studies were 
also performed following the precipitation. The Gibbs free energy change of the reaction is -125.52 $\mathrm{kJ} / \mathrm{mol}$ at the standard state conditions $\left(1 \mathrm{~atm}\right.$ and $25^{\circ} \mathrm{C}$ ). The negative value of $\Delta \mathrm{G}$ indicates the precipitation of thorium dioxide is thermodynamically favorable. The enthalpy value of -108.99 $\mathrm{kJ} / \mathrm{mol}$ further supports and suggests that the reaction is exothermic. The entropy value of thorium precipitation is found to be $70 \mathrm{~J} \cdot \mathrm{mol}^{-1} \cdot \mathrm{K}^{-1}$.

$$
\mathrm{Th}_{(a q)}^{4+}+4 O \mathrm{H}_{(a q)}^{-}=\mathrm{ThO}_{2(s)}+2 \mathrm{H}_{2} \mathrm{O}
$$

Thorium, with its only oxidation state, $4^{+}$, is relatively easy to work with compared to other actinide elements, such as uranium, because of the simplicity of the species (Torapava et al., 2009). Uranium can be seen in many forms, such as U (III, IV, V, VI), and forms various complexes as oxides, phosphates, carbonates, etc. (Navrotsky et al., 2013). U (IV and VI) are the most common and stable states (Monji et al., 2016). The speciation of uranium in chloride media is given in Figure 20. Uranium initially exists in the solution with its $6^{+}$oxidation state as a uranyl cation. According to the literature data, the precipitation of uranium starts at a $\mathrm{pH}$ value of approximately 6 (Zhu et al., 2015), which means the solubility of the ions decreases, and the tendency to create insoluble complexes increases. Langmuir (1978) and Brookins (1988) performed comprehensive studies about the thermodynamic characteristics of aqueous and solid uranium species. Under oxidizing conditions $(\mathrm{pH}<4)$, uranium exists as $\mathrm{UO}_{2}{ }^{2+}(\mathrm{U}(\mathrm{VI}))$, which is well correlated with the results obtained in this study (Langmuir, 1978; Regenspurg et al., 2010; Abdel-Sabour, 2014; Monji et al., 2016). A sharp decrease in the $\mathrm{UO}_{2}{ }^{2+}$ concentration is noticed starting from a $\mathrm{pH}$ value of 5. As the concentrations of $\mathrm{U}^{6+}$ and $\mathrm{UO}_{2}{ }^{2+}$ are lessening, aqueous uranyl hydroxide $\left(\mathrm{UO}_{2}(\mathrm{OH})_{2}\right)$ starts to be formed, which then transfers into a crystalline form around $\mathrm{pH} 7$. Similarly, the precipitation of $\mathrm{UO}_{2}(\mathrm{OH})_{2}$ is spontaneous and exothermic with $\Delta \mathrm{G}$ and $\Delta \mathrm{H}$ values of $-65.69 \mathrm{~kJ} / \mathrm{mol}$ and $-53.93 \mathrm{~kJ} / \mathrm{mol}$, separately, at the standard state conditions. The entropy of the reaction was calculated to be $40 \mathrm{~J} \cdot \mathrm{mol}^{-1} \cdot \mathrm{K}^{-1}$. Similar findings were also seen in Orabi's study (2013).

$$
U O_{2(a q)}^{2+}+2 O H_{(a q)}^{-}=U O_{2}(O H)_{2(s)}
$$




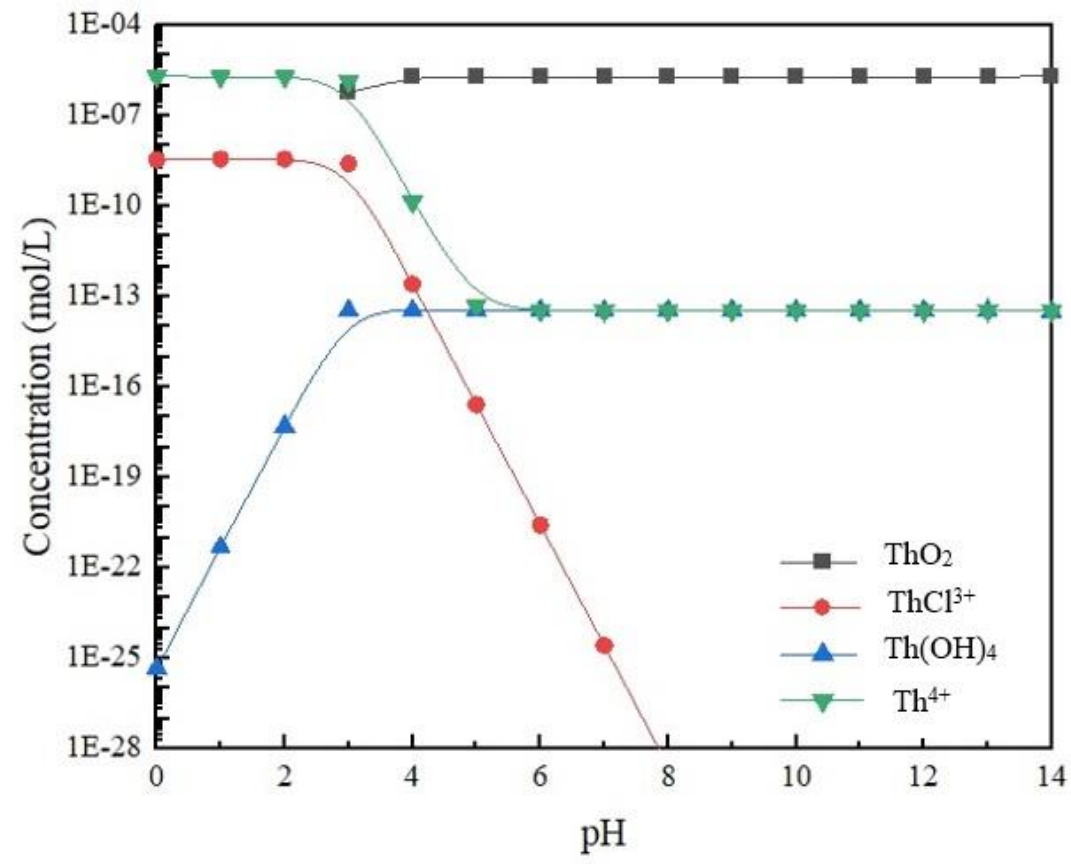

Figure 19 Species distribution diagram of Th- $\mathrm{HCl}$ system.

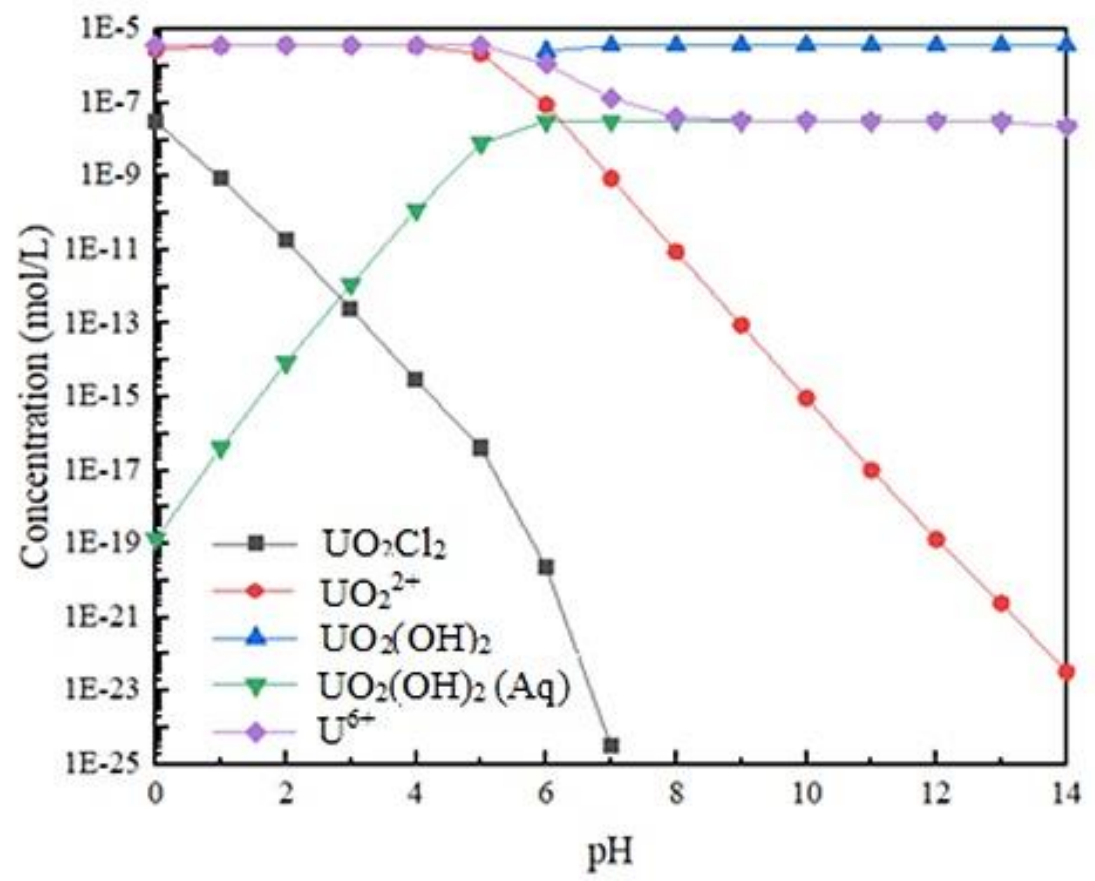

Figure 20 Species distribution diagram of $\mathrm{U}-\mathrm{HCl}$ system. 
Pourbaix diagrams of thorium and uranium systems were generated using HSC Chemistry Software as shown in Figure 21 and Figure 22, respectively. The elements with the highest effect were selected to create a system that is as close as possible to the real system. In the diagrams, the dashed lines represent the stability area of water, and all Eh-pH diagrams are constructed assuming the system is in equilibrium with the water. The redox potential measurements of the synthetic solution at various $\mathrm{pH}$ values are given in Table 14. Based on the redox potential readings, it is clear that at $\mathrm{pH} 4.8$, thorium is stable in the form of $\mathrm{ThO}_{2}$, which is an additional support of the experimental findings and previously presented species distribution diagrams. It initially present as $\mathrm{Th}^{+4}$ which later reacts with $\mathrm{OH}^{-}$as the $\mathrm{pH}$ increases and the diagram moves to the right. On the other hand, according to the diagram, uranium may form a compound with sodium, which is expected due to the high concentration of sodium ions present in the solution.

Table 14 Redox potential measurements at various $\mathrm{pH}$ values

\begin{tabular}{cc}
\hline $\mathrm{pH}$ & Eh $(\mathrm{mV})$ \\
\hline Initial $\mathrm{pH}<0$ & 1030.60 \\
0.00 & 927.00 \\
0.25 & 794.80 \\
0.50 & 687.50 \\
1.00 & 597.90 \\
1.50 & 590.50 \\
2.50 & 564.90 \\
3.00 & 551.40 \\
3.50 & 434.40 \\
4.00 & 389.30 \\
4.50 & 338.80 \\
4.80 & 331.70 \\
5.00 & 296.40 \\
\hline
\end{tabular}




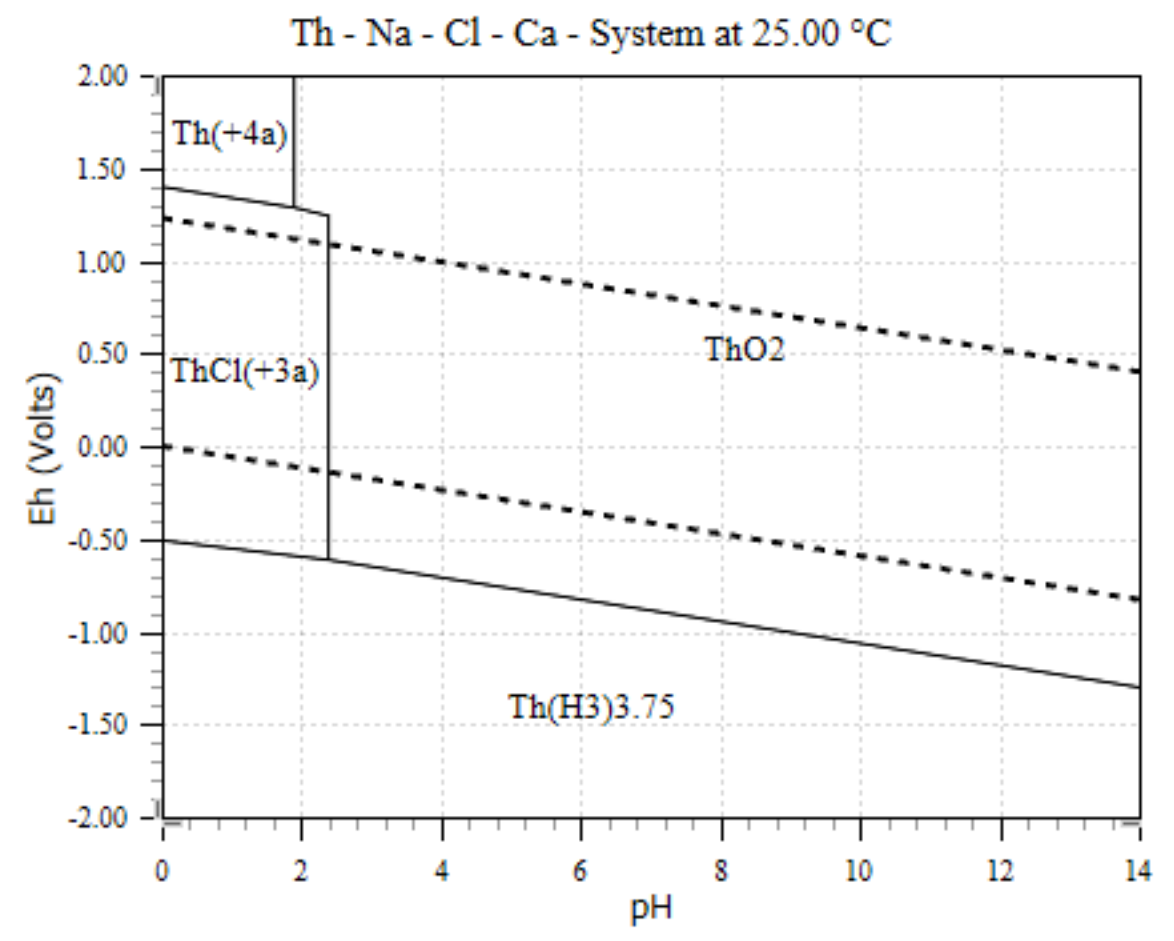

Figure 21 Eh-pH diagram of Th-Cl-Na-Ca- $\mathrm{H}_{2} \mathrm{O}$ system.

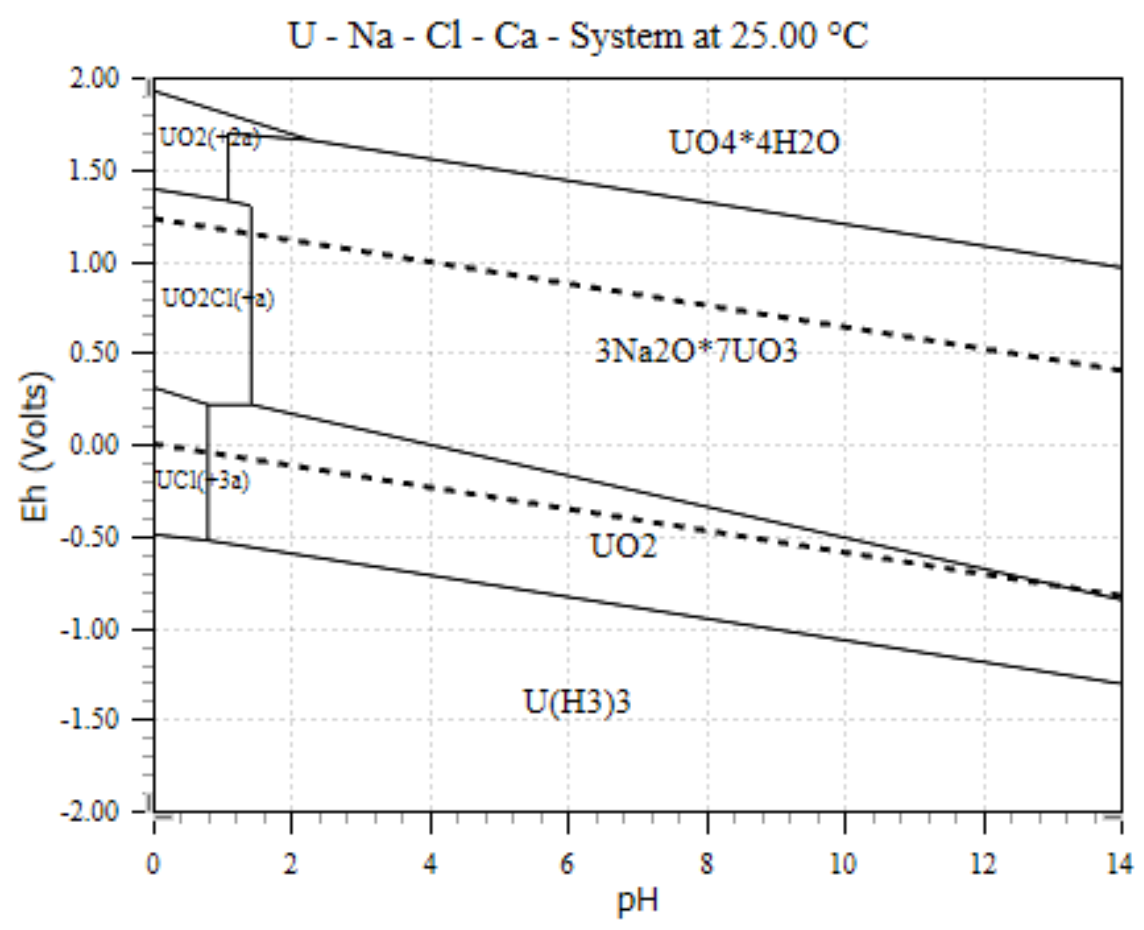

Figure 22 Eh-pH diagram of U-Cl-Na-Ca- $\mathrm{H}_{2} \mathrm{O}$ system 
The species distribution diagrams of selected rare earth elements are shown in Figure 23. Considering the concentration of individual rare earths in the synthetic feedstock solution, five rare earth elements, including cerium, neodymium, gadolinium, lanthanum selected to represent different rare earth groups (i.e., heavy (Y, Eu, Gd, Tb, Dy, Ho, Er, Tm, Yb, Lu), light (La, Ce, Pr, $\mathrm{Nd}, \mathrm{Sm}$ ), critical (Y, Nd, Tb, Dy, Eu), and uncritical (La, Ce, Pr, Sm, Gd, Ho, Er, Tm, Yb, Lu) rare earth elements). It is known that most rare earths existed in the synthetic solution as REEs ${ }^{3+}$. However, cerium, praseodymium, and terbium may also be tetravalent, and samarium, europium, and ytterbium in a divalent state, but these states are not stable (Thakur, 2000). Rare earths become less soluble with increasing $\mathrm{pH}$, and thermodynamically stable rare earth hydroxides can be obtained by treating aqueous solutions with a base. The precipitation of rare earths occurs between pH 6.8-8, where there is an overlap with uranium that leads to the co-precipitation of the two (Zhu et al., 2015). Rare earths' resistance to the base and the solubility decreases from light rare earths towards heavy rare earths, with lanthanum being the most basic and soluble.

As seen in the diagrams (Figure 23), the precipitation behavior of rare earths is alike, reflecting the similarity in their physical and chemical properties (Al-Nafai, 2015). Similar types of species tend to be formed approximately at the same $\mathrm{pH}$ regions. For all the rare earth elements examined, an increase in the concentration of rare earth hydroxide compound is observed starting from a $\mathrm{pH}$ value of 8 . An immediate increase in the concentration of $\operatorname{REE}(\mathrm{OH})_{3}(\mathrm{aq})$ is also seen for all of the selected elements which is then stabilize around a $\mathrm{pH}$ value of 8 . A slight increase in the concentration of trivalent rare earth ions is observed around $\mathrm{pH} 10$, which shows that the solubility starts again at high $\mathrm{pH}$ regions. Thermodynamic values were also calculated for rare earths and can be seen in Table 15. Due to their similar characteristics, the thermodynamic values were considerably close to each other. The precipitation reaction of rare earths (Eq. 25) is favored according to the Gibbs free energy values. The enthalpy changes are suggesting that the processes are exothermic. Moreover, the thermodynamic findings shown in Table 15 indicate that heavy rare earth elements tend to precipitate as opposed to light elements. A more negative $\Delta \mathrm{G}$ value is obtained for heavy rare earth elements (i.e., Gadolinium and Yttrium) than light rare earth elements (i.e., Cerium, Neodymium, and Lanthanum).

$$
\operatorname{REEs}_{(a q)}^{3+}+3 O H_{(a q)}^{-}=\operatorname{REEs}(\mathrm{OH})_{3}
$$

Table 15 Gibbs free energy and enthalpy and entropy values of rare earths under standard operating conditions ( $1 \mathrm{~atm}$ and $25^{\circ} \mathrm{C}$ ).

\begin{tabular}{c|c|c|c}
\hline Elements / Thermodynamic Parameter & $\Delta \mathrm{G}(\mathrm{kJ} / \mathrm{mol})$ & $\Delta \mathrm{H}(\mathrm{kJ} / \mathrm{mol})$ & $\Delta \mathrm{S}\left(\mathrm{J} \cdot \mathrm{mol}^{-1} \cdot \mathrm{K}^{-1}\right)$ \\
\hline Cerium & -130.5 & -32.4 & +330 \\
Neodymium & -136.9 & -29.4 & +360 \\
Gadolinium & -143.6 & -32.9 & +370 \\
Lanthanum & -124.6 & -19.7 & +350 \\
Yttrium & -173.5 & -59.3 & +380 \\
\hline
\end{tabular}



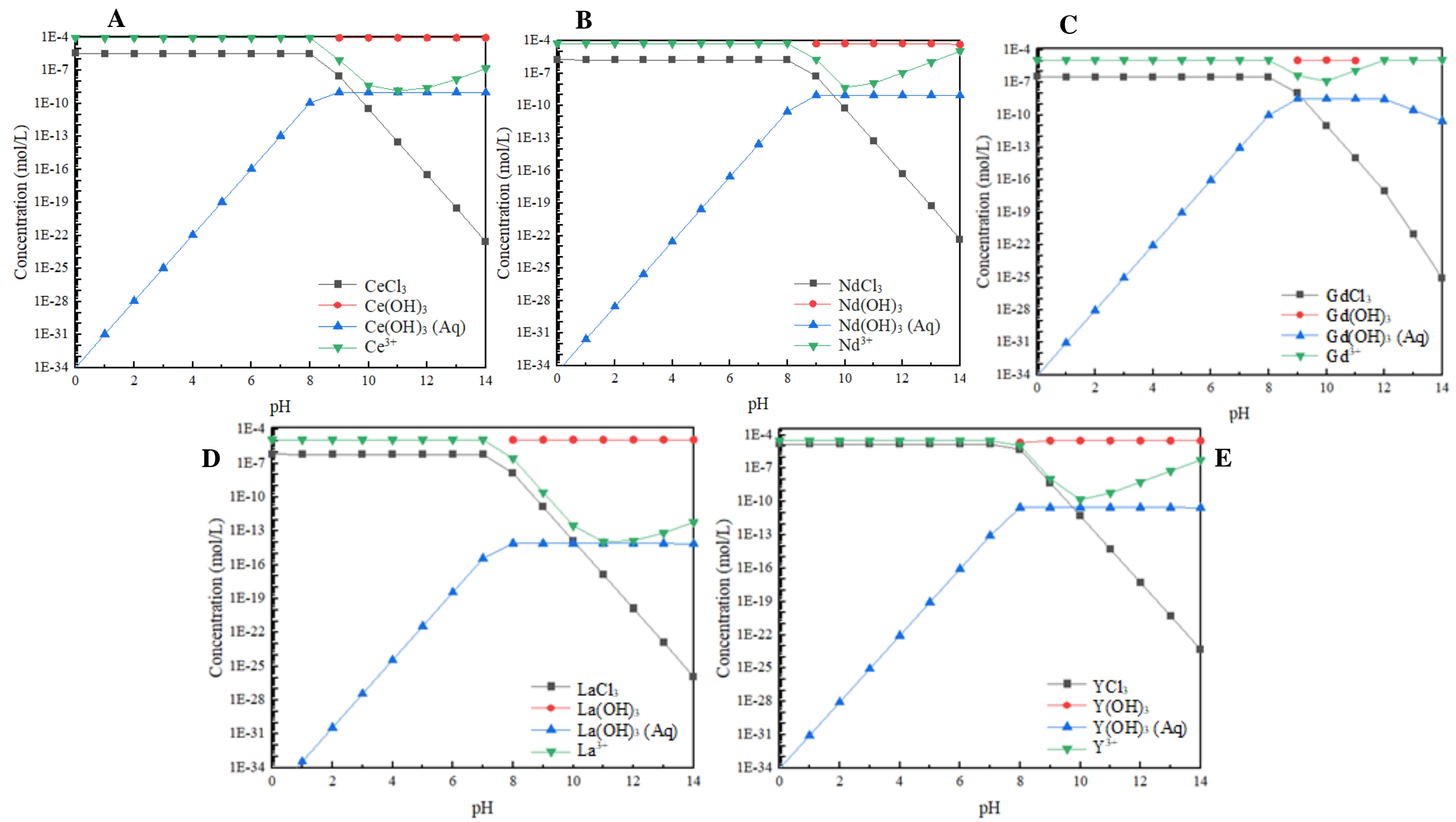

Figure 23 Species distribution diagrams of REEs-HCl system. A) Cerium, B) Neodymium, C) Gadolinium, D) Lanthanum, E) Yttrium 
The speciation of iron and aluminum in the chloride media is presented in Figure 24 and Figure 25 , respectively. Also, their precipitation reactions are given in Eqs. (26) and (27). In the synthetic feedstock solution, iron exists with its trivalent oxidation state, $\mathrm{Fe}^{3+}$. The concentration of $\mathrm{Fe}^{+3}$ starts to decrease around a $\mathrm{pH}$ value of 3 , which is consistent with the literature data (Balintova and Petrilakova, 2011; Seo et al., 2017). Due to the nature of the synthetic solution's medium, iron (III) chloride $\left(\mathrm{FeCl}_{3}\right)$ is also seen in the solution, and its concentration starts to decrease at the same $\mathrm{pH}$ value. While the concentrations of $\mathrm{Fe}^{+3}$ and $\mathrm{FeCl}_{3}$ are reducing, the formation of iron hydroxide $\left(\mathrm{Fe}(\mathrm{OH})_{3}\right)$ is seen. Iron precipitation is found to be thermodynamically stable with a corresponding $\Delta \mathrm{G}$ value of $-317.13 \mathrm{~kJ} / \mathrm{mol}$ and $\Delta \mathrm{H}$ value of $-229.43 \mathrm{~kJ} / \mathrm{mol}$. According to previous selective precipitation test results, approximately $66 \% \mathrm{Fe}$ precipitated out at $\mathrm{pH} 4.85$. Characterization studies also supported the presence of iron in the precipitates. XRD revealed the formation of iron oxide and the iron peak was detected in the EDX spectrum as well, which were all consistent with the distribution of iron species as indicated in Figure 24.

When an alkali is added to a solution containing aluminum, a precipitate is obtained in a hydroxide gel, $\mathrm{Al}(\mathrm{OH})_{3}$. Generally, $\mathrm{Al}(\mathrm{OH})_{3}$ formation starts to be seen at a $\mathrm{pH}$ value of 5 (Balintova and Petrilakova, 2011), which is supported by Figure 25. The original form of aluminum, $\mathrm{Al}^{3+}$, gradually transforms into $\mathrm{Al}(\mathrm{OH})_{2}{ }^{+1}$. However, due to continuous reactions occurring between aluminum species and $\mathrm{OH}^{-}$, the formed $\mathrm{Al}(\mathrm{OH})_{2}{ }^{+1}$ is not stable, and it decreases significantly at a $\mathrm{pH}$ value of 4 . At that $\mathrm{pH}$, the formation of $\mathrm{Al}(\mathrm{OH})_{3}$ initiates. On the other hand, in the presence of sufficiently alkaline solutions, aluminum decomposes water with the evolution of hydrogen, dissolving as aluminate ions $\mathrm{AlO}_{2}^{-}$(Pourbaix, 1974). Although stability is observed for $\mathrm{Al}(\mathrm{OH})_{3}$ through a wide range of $\mathrm{pH}$ values, a decrease in its concentration starts when $\mathrm{pH}$ exceeds 10 . Like iron, the aluminum precipitation mechanism is thermodynamically favorable with a standard energy change value of $-540.03 \mathrm{~kJ} / \mathrm{mol}$. Moreover, the enthalpy change, $-545.53 \mathrm{~kJ} / \mathrm{mol}$, suggests an exothermic reaction.

$$
\begin{aligned}
& \mathrm{Fe}_{(a q)}^{3+}+3 O H_{(a q)}^{-}=\mathrm{Fe}(\mathrm{OH})_{3(s)} \\
& A l_{(a q)}^{3+}+3 O H_{(a q)}^{-}=\mathrm{Al}(\mathrm{OH})_{3(s)}
\end{aligned}
$$




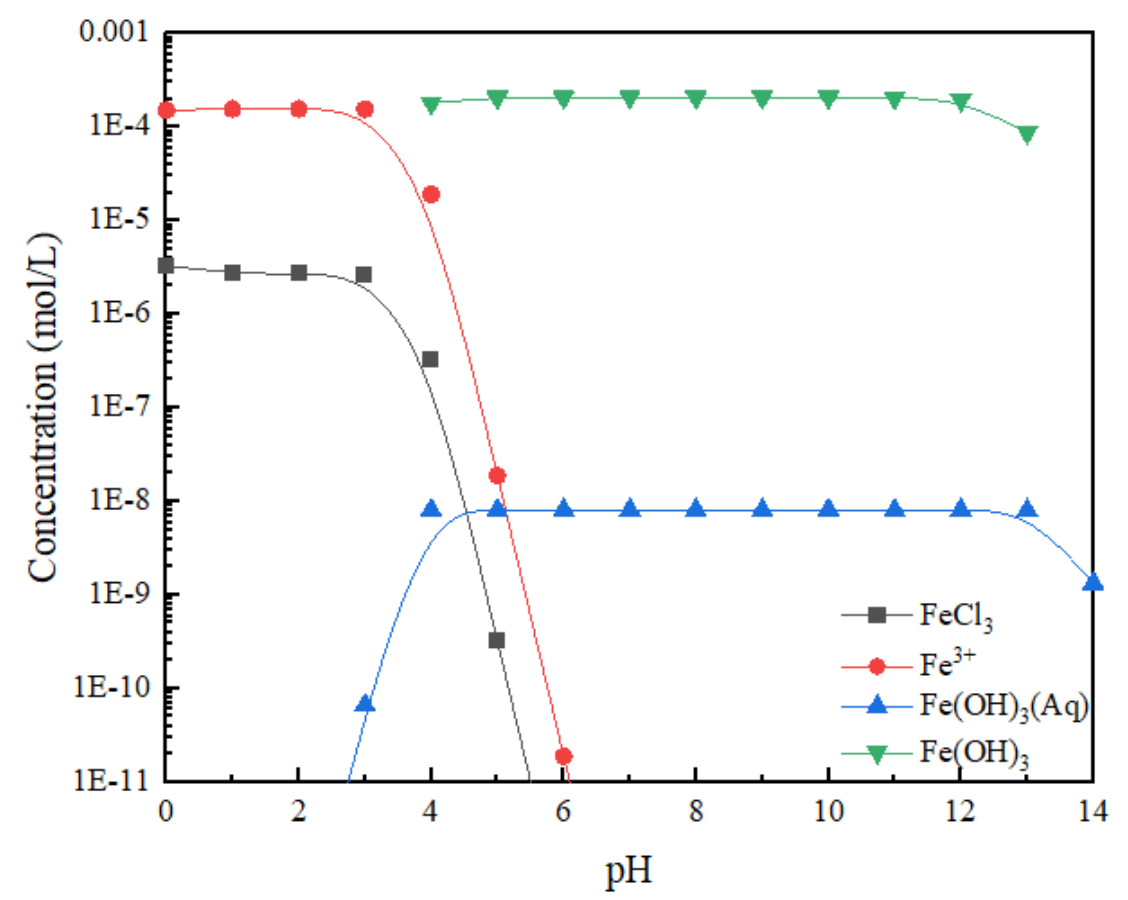

Figure 24 Species distribution diagram of $\mathrm{Fe}-\mathrm{HCl}$ system.

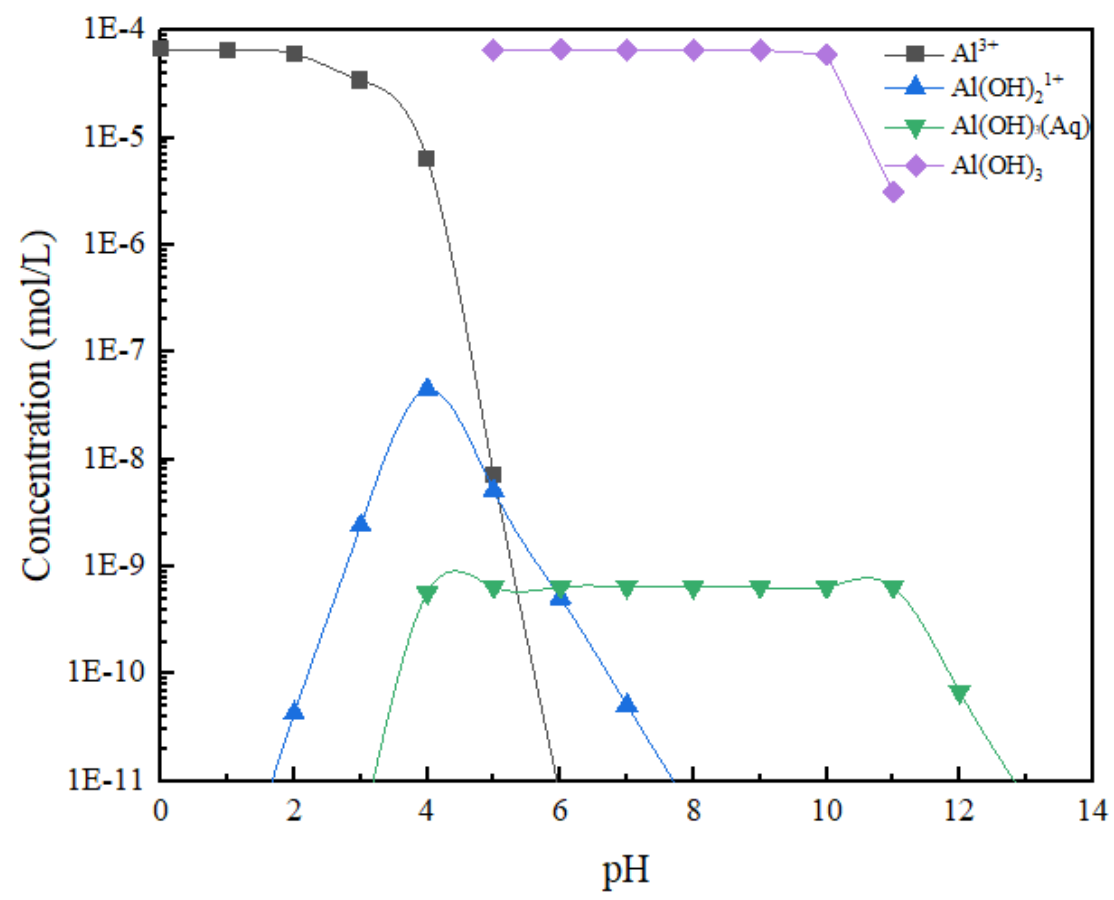

Figure 25 Species distribution diagram of $\mathrm{Al}-\mathrm{HCl}$ system. 


\subsubsection{The First Subset of Solvent Extraction Tests}

The experimental flowsheet, including the operational conditions, is given in Figure 26. Recovery of rare earths and uranium into the final rare earth product stream, based solely on solvent extract circuit, as a result of the 15 tests are given in Figure 27. Likewise, the overall recovery of rare earths and uranium is shown in Figure 28, taking both selective precipitation and solvent extraction into account.

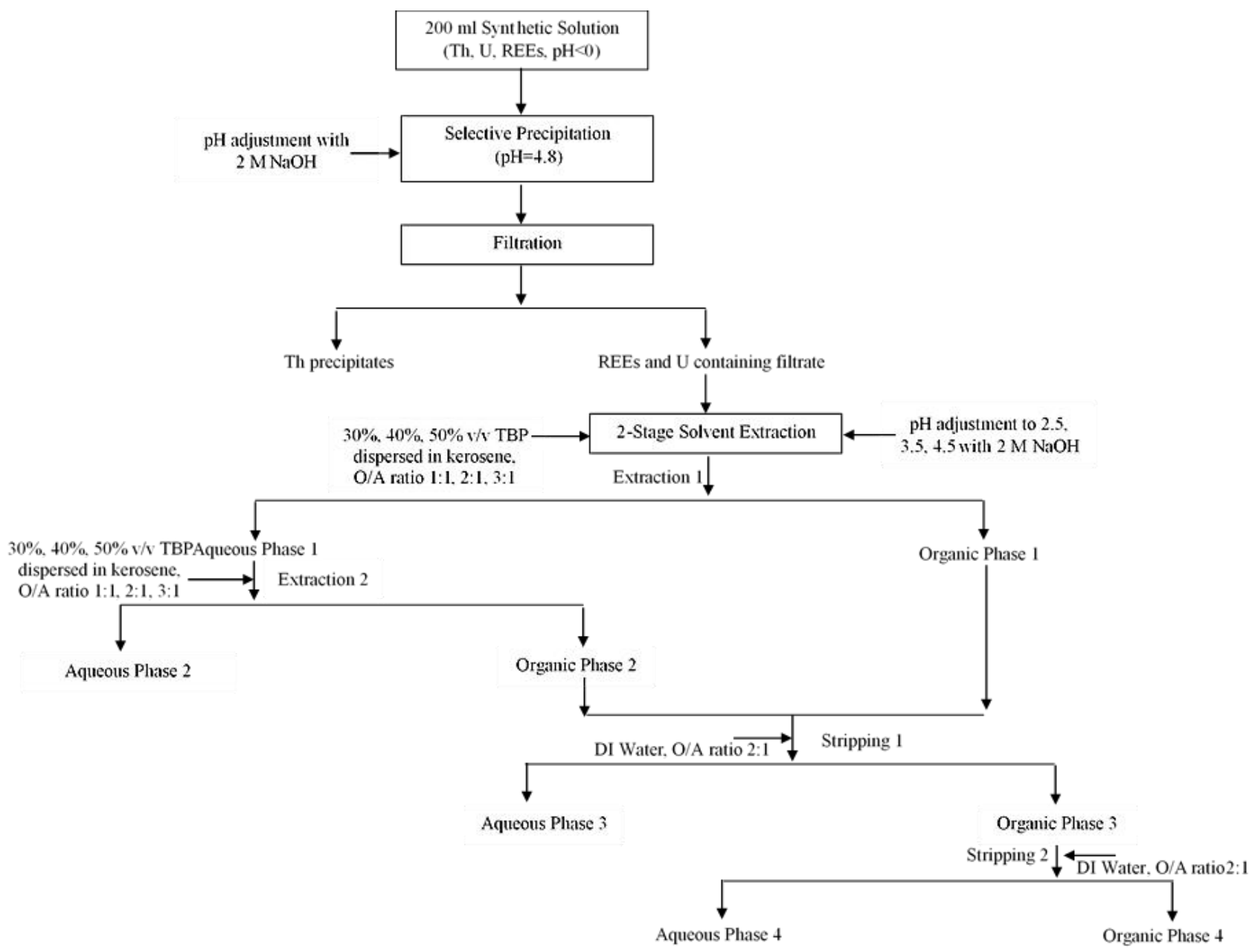

Figure 26 Experimental procedure and the operating conditions followed the second subset of solvent extraction tests.

As seen in Figure 27, the rare earth recovery obtained from the solvent extraction circuit varied from $90 \%$ to $98.9 \%$, while the corresponding uranium recovery changed between $5.9 \%$ to $89.4 \%$. This noteworthy fluctuation in uranium recovery implies that the chosen variables had an impact on the removal of uranium. On the other hand, the rare earth recovery value close to each other suggests that the same variables had less profound or a minimal impact on the recovery of rare earths. 
Figure 28 further indicates the overall recovery of rare earths and uranium based on the whole experimental route by incorporating the selective precipitation stage. After considering the amounts of rare earths and uranium that precipitated out as solid forms, the overall recovery of rare earths and uranium both decreased to a value varying between $72.6 \%-79.8 \%$ and $3.1 \%-46.5 \%$, respectively. Data from Figure 27 and Figure 28 indicate the best results with respect to the recovery of rare earth and uranium were obtained by Test 8 under the following conditions: $50 \mathrm{v} \%$ $\mathrm{TBP}$, feed $\mathrm{pH}$ value of 3.5, an $\mathrm{O} / \mathrm{A}$ ratio at 3 . An overall rare earth recovery of approximately $79.8 \%$ with a corresponding uranium recovery of $3.1 \%$ was produced under the same operating conditions. The elemental composition (i.e., individual rare earth and uranium) of the final rare earth product stream obtained from Test 8 is also given in Table 16. No thorium was reported since all the thorium precipitated out at a $\mathrm{pH}$ of approximately 4.8 prior to the solvent extraction tests.

It was also observed that a noticeable portion of uranium was reported to the organic stream instead of the uranium product stream. For example, for Test 8 , on the basis of the solvent extraction circuit, the recovery of uranium into the uranium product stream and final rare earth product stream was $52.1 \%$ and $5.9 \%$, respectively. In contrast, the remaining uranium reported to the organic stream, which needs to be further extracted so that the organic can be reused for solvent extraction from an economic standpoint. One solution to purify TBP is treating the organic stream with sodium carbonate or sodium hydroxide solutions to neutralize and precipitate the organic impurities with high molecular weights (Geier, 1979; Srinivas et al., 1994; International Atomic Energy Agency, 2000). The quality of the organic phase must be periodically checked and maintained after recycling since TBP may be subject to hydrolyze in the aqueous phases (International Atomic Energy Agency, 2000). Among the 15 tests, the amount of rare earths reporting to the organic stream was $>1 \%$ for eight tests, with the highest value of $5.3 \%$ corresponding to Test 8 . For the remaining seven tests, the non-selective recovery of rare earths into the organic stream is all less than $1 \%$, which is negligible.

Detailed results of the individual rare earth elements and uranium extraction recoveries (\%), their concentrations in the rare earth product stream, as well as the detailed overall extraction recoveries are given in Table 29, Table 30, and Table 31 separately in the Appendix. 
Table 16 Elemental composition of individual rare earth and uranium (mg/L) in the final REE product stream obtained from Test 8 .

\begin{tabular}{|c|c|c|c|c|c|c|c|c|c|c|c|c|c|c|c|c|}
\hline Sc & $\mathrm{Y}$ & $\mathrm{La}$ & $\mathrm{Ce}$ & $\operatorname{Pr}$ & $\mathrm{Nd}$ & $\mathrm{Sm}$ & $\mathrm{Eu}$ & $\mathrm{Gd}$ & $\mathrm{Tb}$ & Dy & Ho & $\mathrm{Er}$ & $\mathrm{Tm}$ & $\mathrm{Yb}$ & $\mathrm{Lu}$ & $\mathrm{U}$ \\
\hline 0.00 & 0.06 & 0.04 & 0.30 & 0.03 & 0.17 & 0.08 & 0.01 & 0.04 & 0.03 & 0.02 & 0.00 & 0.01 & 0.00 & 0.00 & 0.00 & 0.001 \\
\hline
\end{tabular}

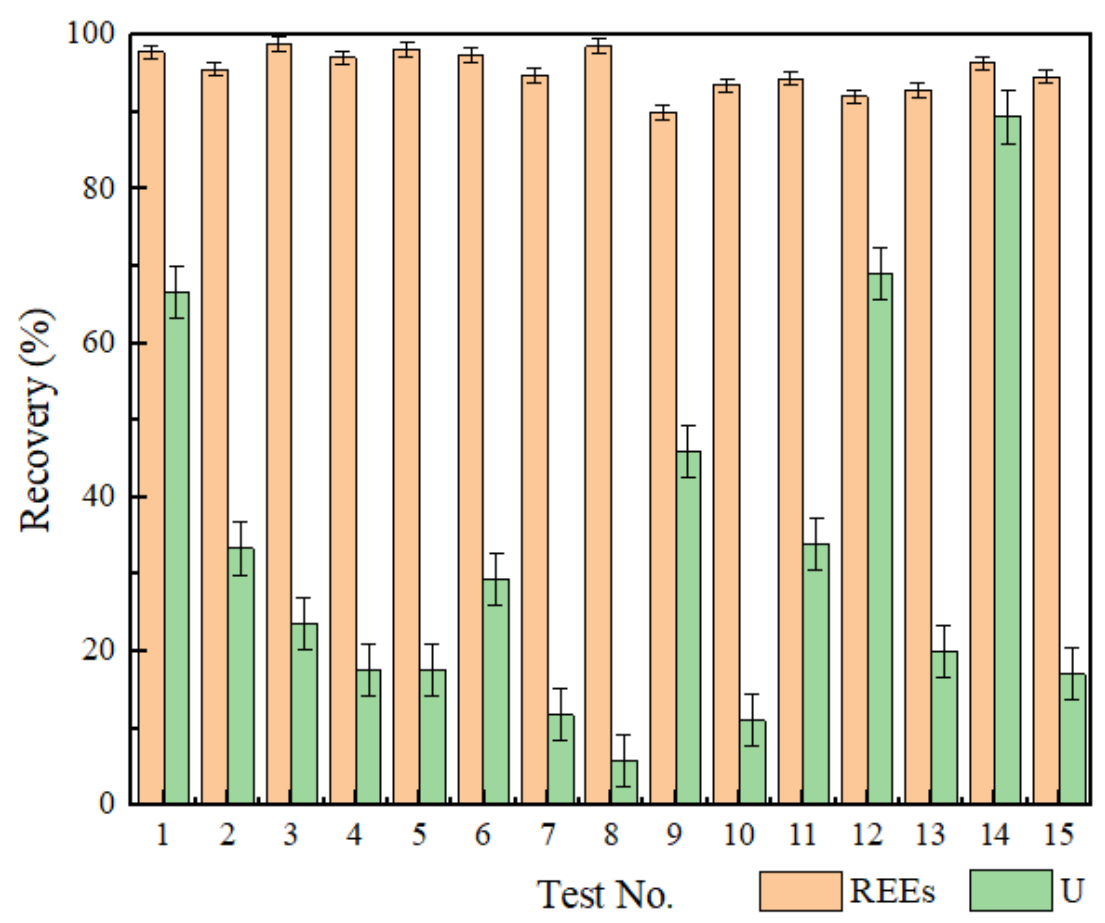

Figure 27 Recovery of total rare earths and uranium into the final REE product stream based solely on solvent extraction circuit. Error bars represent one standard deviation of three replicate tests. 


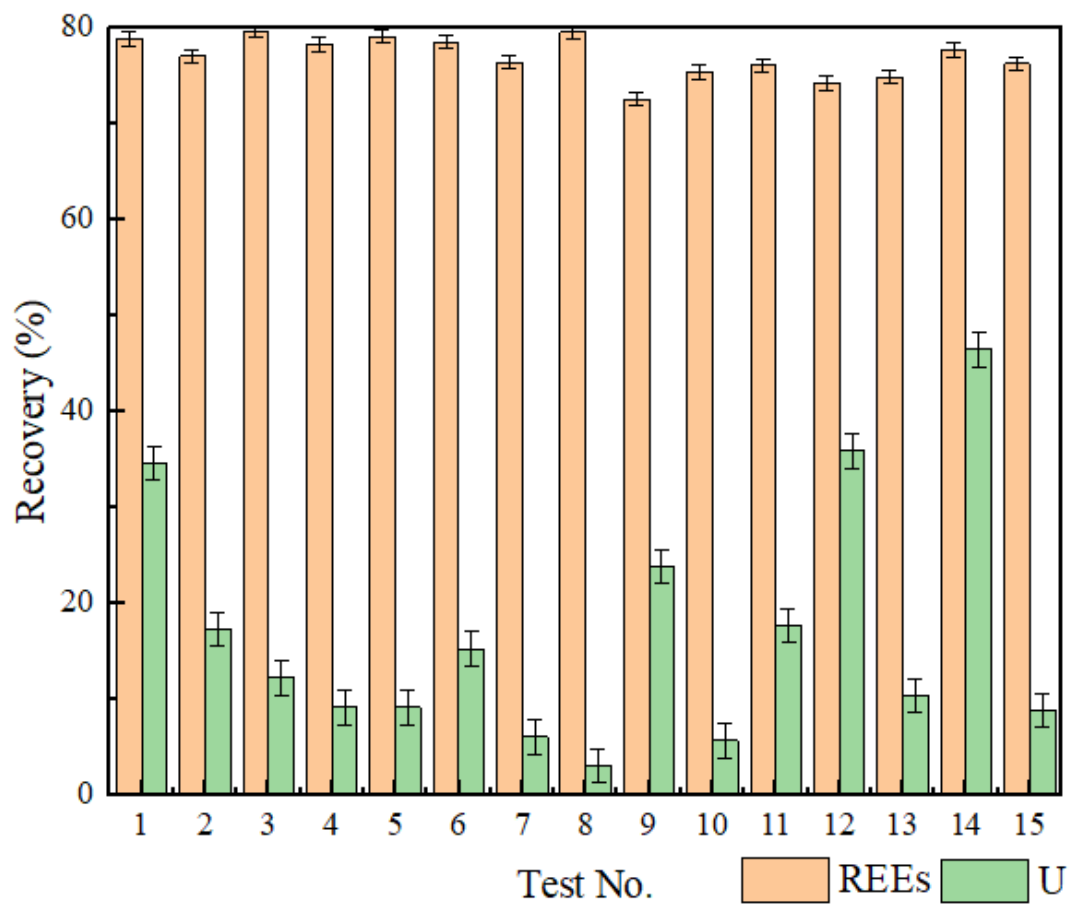

Figure 28 Overall recovery of total rare earths and uranium with respect to the overall experimental flowsheet. Error bars represent one standard deviation of three replicate tests.

\subsubsection{Statistical Analysis and Modeling of First Subset of Solvent Extraction Tests}

Following data analysis, the impact of the operating variables (i.e., extractant concentration, feed solution $\mathrm{pH}$, and $\mathrm{O} / \mathrm{A}$ ratio) on the recovery of rare earth and uranium were analyzed using the Design Expert statistical analysis software.

The statistical model developed to predict the recovery of uranium by considering the influential operating parameters is shown in equation 28. It can be seen from the quadratic model that all three variables have a significant impact on the non-selective recovery of uranium into the REE product stream.

Uranium Recovery $=+596.01-12.38 * A-85.85 * B-110.80 * C+0.65 * A B+0.80 *$ $A C+3.92 * B C+0.09 * A^{2}+4.81 * B^{2}+10.88 * C^{2}$

where the uncoded model term $\mathrm{A}, \mathrm{B}$, and $\mathrm{C}$ is corresponding to extractant concentration, feed solution $\mathrm{pH}$, and $\mathrm{O} / \mathrm{A}$ ratio, respectively.

The Analysis of Variance (ANOVA) table provided detailed information on the significance of the model, as shown in Table 17. The Fischer variation ratio (F-value) is a statistically valid measure of how well the factors describe the variation in the data about its mean. In this model, an F-value of 9.84 implies the model is significant and the probability of an F-value to become this large is $1.07 \%(<5 \%$, which is the critical P-value used to determine the significance). Model terms are considered significant within a $95 \%$ confidence level, or in other words, values of "prob $>F$ " less 
than 0.05 indicate that terms are significant. Terms A (extractant concentration), B (pH), and C (O/A ratio) are all significant model terms according to the data obtained from ANOVA, and they all have a profound impact on uranium recovery. The "lack-of-fit" model is insignificant based on a p-value greater than 5\%, which further supports the statistical significance of the model. An Rsquared value of 0.9466 and an adjusted R-squared value of 0.8503 again validate the model. Even though the coefficients associated with high-order terms are not high compared to that of the firstorder terms, they are still needed to justify the significance of the model.

Table 17 ANOVA analysis of the quadratic model for the prediction of uranium recovery.

\begin{tabular}{ccccccc}
\hline Source & $\begin{array}{c}\text { Sum of } \\
\text { Squares }\end{array}$ & df & $\begin{array}{c}\text { Mean } \\
\text { Square }\end{array}$ & F Value & $\begin{array}{c}\text { p-value } \\
\text { Prob> F }\end{array}$ & \\
\hline Model & 7992.72 & 9 & 888.08 & 9.84 & 0.0107 & Significant \\
Lack of Fit & 428.26 & 3 & 142.75 & 12.39 & 0.0756 & Not significant \\
A-Extractant Concentration & 1214.51 & 1 & 1214.51 & 13.46 & 0.0145 & \\
B-pH & 1890.82 & 1 & 1890.82 & 20.95 & 0.0060 & \\
C-O/A Ratio & 3666.25 & 1 & 3666.25 & 40.62 & 0.0014 & \\
AB & 169 & 1 & 169 & 1.87 & 0.2295 & \\
AC & 258.41 & 1 & 258.41 & 2.86 & 0.1514 & \\
BC & 61.54 & 1 & 61.54 & 0.68 & 0.4466 & \\
A & 304.75 & 1 & 304.75 & 3.38 & 0.1256 & \\
B & 85.25 & 1 & 85.25 & 0.94 & 0.3758 & \\
C & 436.87 & 1 & 436.87 & 4.84 & 0.0791 & \\
R-Squared & 0.9466 & & & & & \\
Adj R-Squared & 0.8503 & & & & & \\
Pred R-Squared & 0.1824 & & & & & \\
Adeq Precision & 10.172 & & & & & \\
\hline
\end{tabular}

3D response surface and 2D contour plots shown in Figure 29 and Figure 30 indicating the impact of two input variables on uranium recovery, while the level of the third variable was fixed at the middle-range value. Figure 29(A) first shows the effect of extractant concentration and feed solution $\mathrm{pH}$ on uranium recovery while the $\mathrm{O} / \mathrm{A}$ ratio was maintained as a constant value of 2 . As seen, a high $\mathrm{pH}$ value and high extractant concentration tend to generate enhanced separation results. The experimental results corroborated that the lowest recovery of uranium into the rare earth product stream (i.e., 5.9\%) was obtained with 50\% TBP by volume. For the tests conducted with low TBP concentrations and low $\mathrm{pH}$ values, the amount of uranium reporting to the final product stream increased substantially. Similarly, the effect of extractant concentration and O/A ratio on uranium recovery was indicated in Figure 29(B) with a constant feed solution $\mathrm{pH}$ value of 3.5 . 
The uranium recovery decreases with an elevation in both extractant concentration and O/A ratio, which is supported by experimental results obtained from Test 8 (i.e., $50 \mathrm{v} \% \mathrm{TBP}, \mathrm{O} / \mathrm{A}$ of 3 ) and Test 14 (i.e., 30 v\% TBP, O/A of 1) where the lowest and highest uranium recovery was achieved, respectively. As the $\mathrm{O} / \mathrm{A}$ ratio and $\mathrm{pH}$ increase, the recovery of uranium into the final product stream decreases, as reflected by Figure 29(C). The shape of contour plots, on the other hand, suggests the degree of interaction between the variables taken into account. Elliptical contours indicate strong interaction, whereas circular contours display less significant or minimum interaction. It can be seen from Figures 30(A), (B), and (C) that feed solution pH and extractant concentration are two variables strongly interacting with each other, as indicated by the elliptical contour plot.

Following uranium, statistical analysis was also performed for the recovery of total rare earths, and a model was first developed to predict the response parameter of interest. However, a scrutiny of the model, as reflected by the ANOVA table, indicates the model is not statistically meaningful. The insignificance of this model is consistent with the experimental findings that the recovery of rare earths did not change much during the solvent extraction stage, with the value varying between $90 \%$ and $98.9 \%$. 

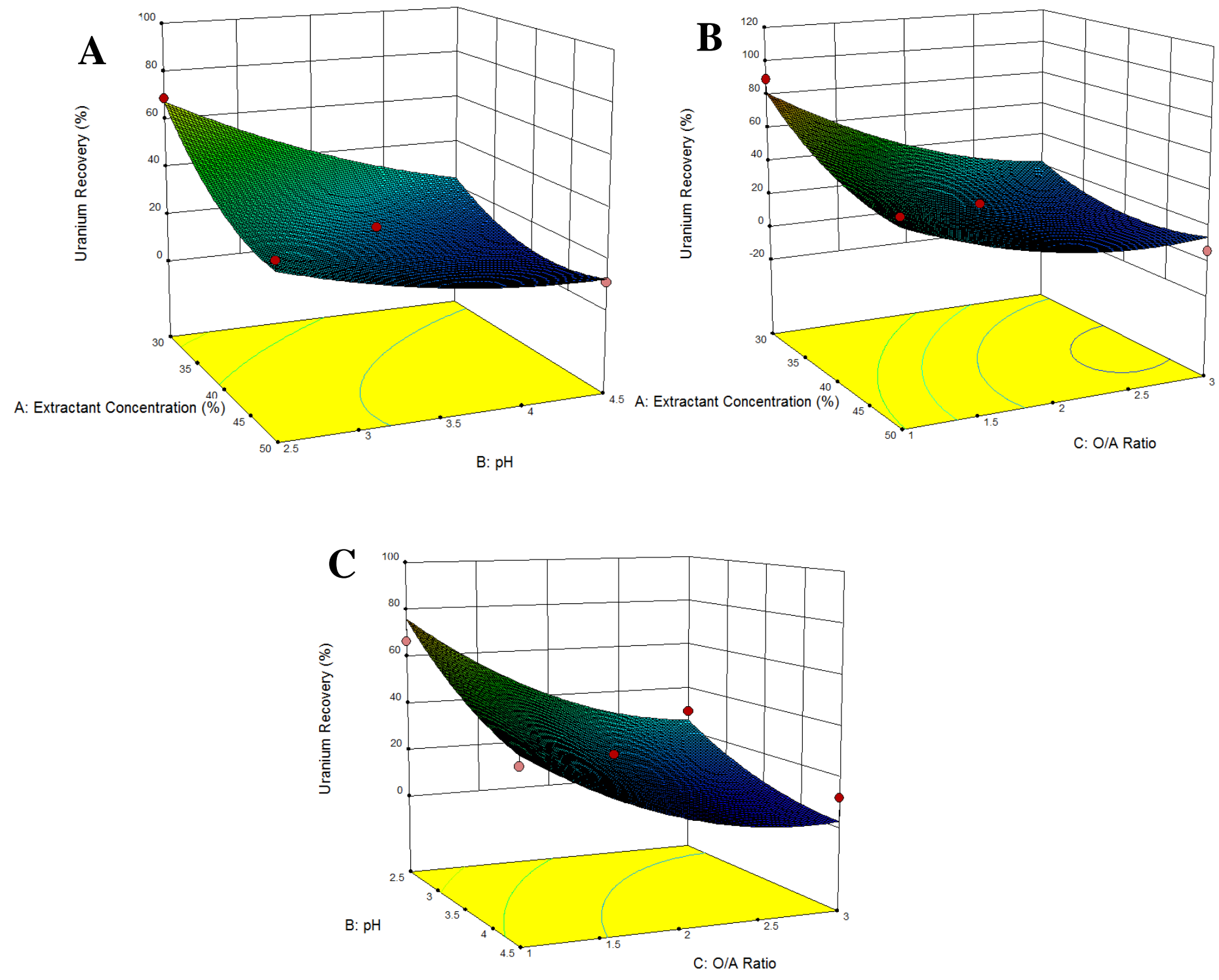

Figure 29 3D surface response graphs obtained from the statistical analysis using Design-Expert. 


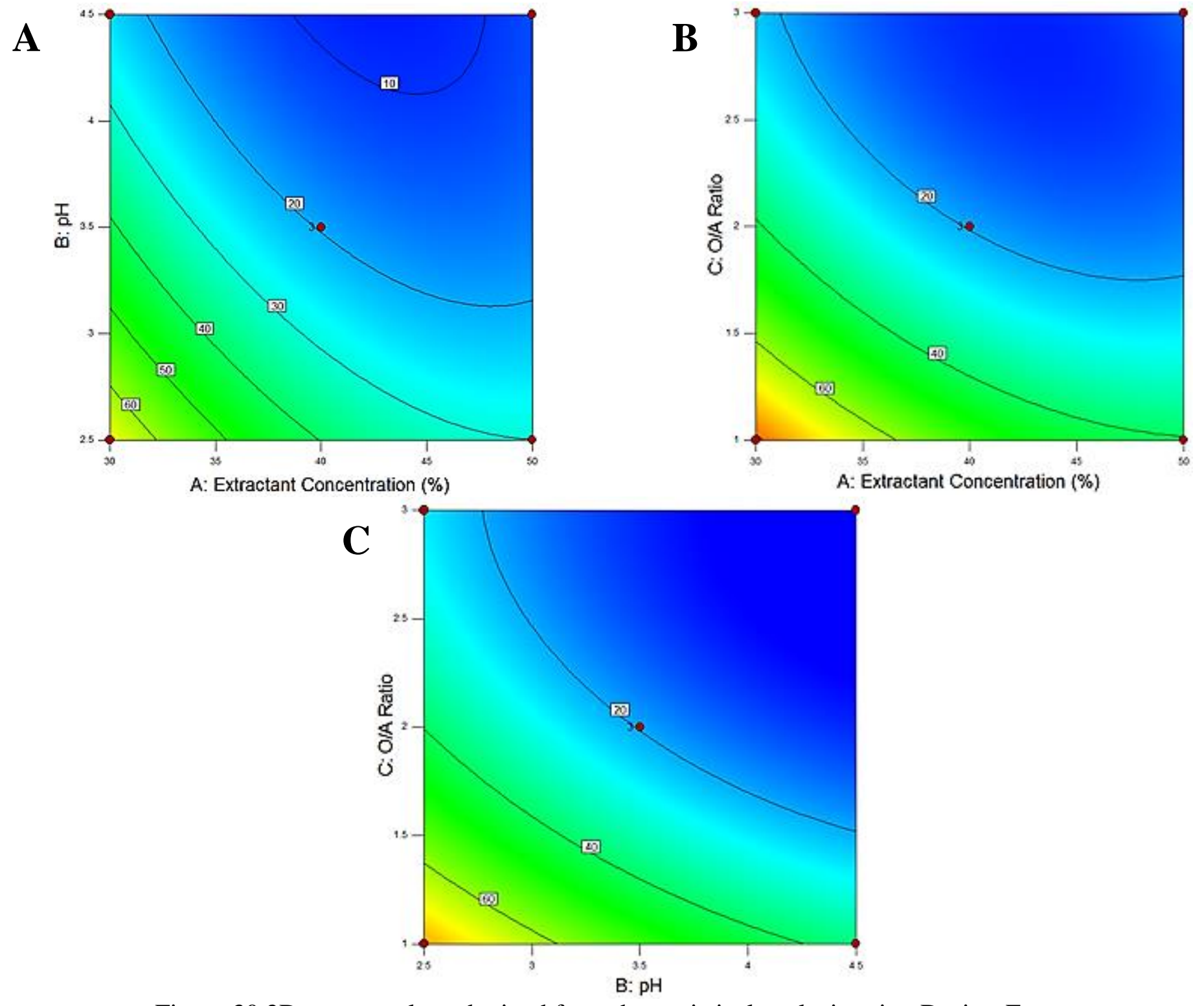

Figure 30 2D contour plots obtained from the statistical analysis using Design-Expert. 


\subsubsection{The Second Subset of Solvent Extraction Tests}

The experimental procedure followed during the study is given in Figure 31 with operating details. Recovery of rare earths and uranium into the final rare earth product stream, based solely on solvent extraction circuit, as a result of 27 tests, are given in Figure 32. Likewise, the overall recovery of rare earths and uranium, taking into account both selective precipitation and solvent extraction, is shown in Figure 33.

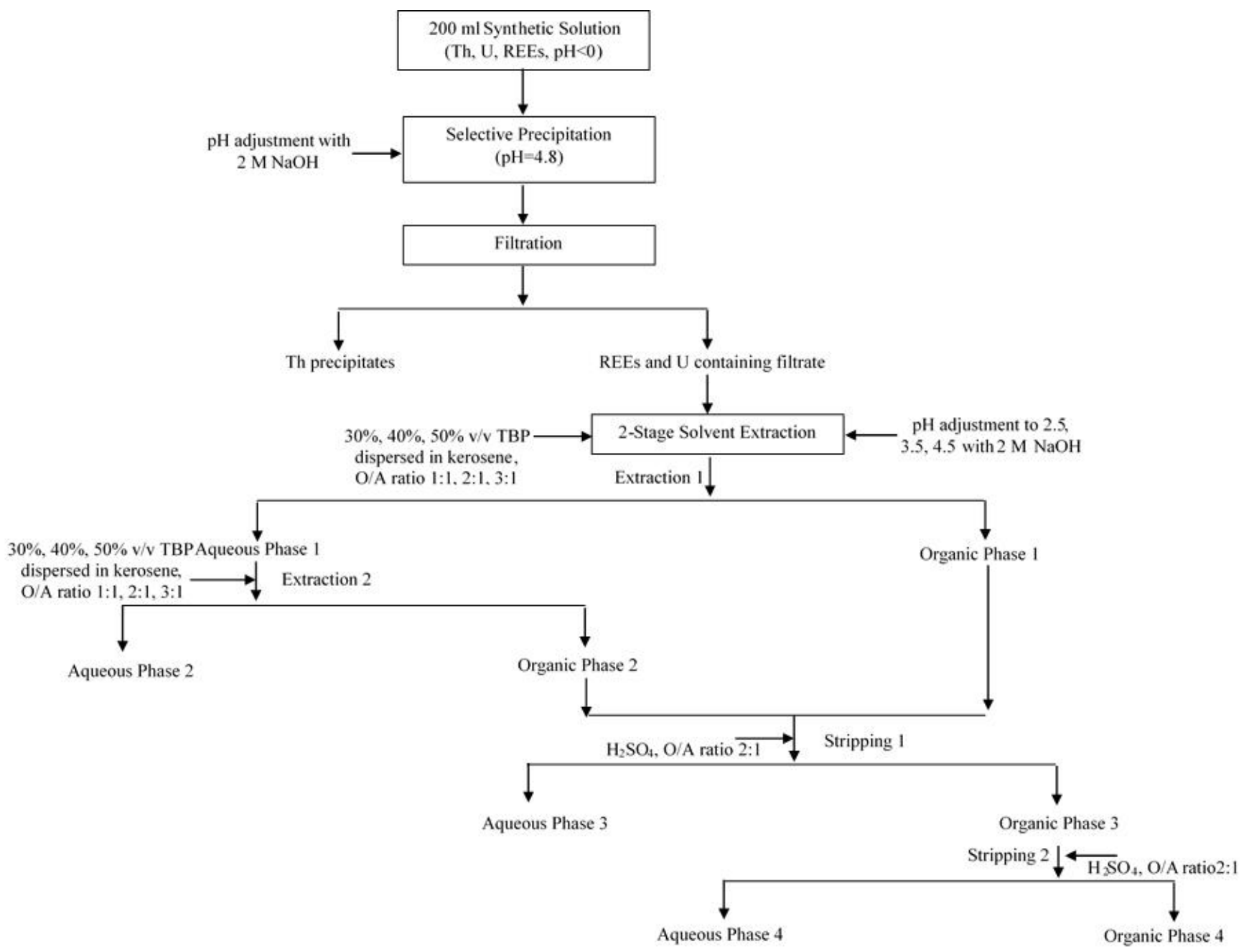

Figure 31 Experimental procedure and the operating conditions followed the second subset of solvent extraction tests.

As seen in Figure 32, the rare earth recovery obtained from the solvent extraction circuit varied from $80.2 \%$ to $97.6 \%$, while the corresponding uranium recovery changed between 3.4 to $62.5 \%$. Similar fluctuation in uranium recovery was also observed in the first set of solvent extraction tests, which implies again that the chosen variables had an impact on the uranium recovery. Moreover, compared to the first set of tests, there is a noticeable reduction in the recovery range of uranium. During the first set of tests, the uranium recovery into the final product stream was 
varying between $5.9 \%$ to $89.4 \%$. The narrowing recovery range shows that the strippant used in the second set of tests had a positive impact on the uranium recovery since the only varying parameter between the first and second set is the strippant.

On the other hand, a much smaller variation in the recovery of rare earths and the value close to each other indicates that the chosen variables had a less profound impact on the recovery of rare earths. A similar phenomenon was also observed in the first set of tests. Although this time, the recovery range of rare earths is slightly wider, which indicates that the new strippant (i.e., $\mathrm{H}_{2} \mathrm{SO}_{4}$ ) may impact the recovery of rare earths.

Figure 33 further indicates the overall recovery of rare earths and uranium based on the whole experimental route by incorporating the selective precipitation stage. After considering the amount of rare earths and uranium previously precipitated out as solid forms, the overall recovery of rare earths and uranium both decreased, ranging from $64.7 \%$ to $78.7 \%$ and $1.8 \%$ to $32.5 \%$, respectively. Detailed results of the individual rare earth elements and uranium extraction recoveries (\%), their concentrations in the rare earth product stream, as well as the detailed overall extraction recoveries are given in Table 32, Table 33, and Table 34, separately in the Appendix.

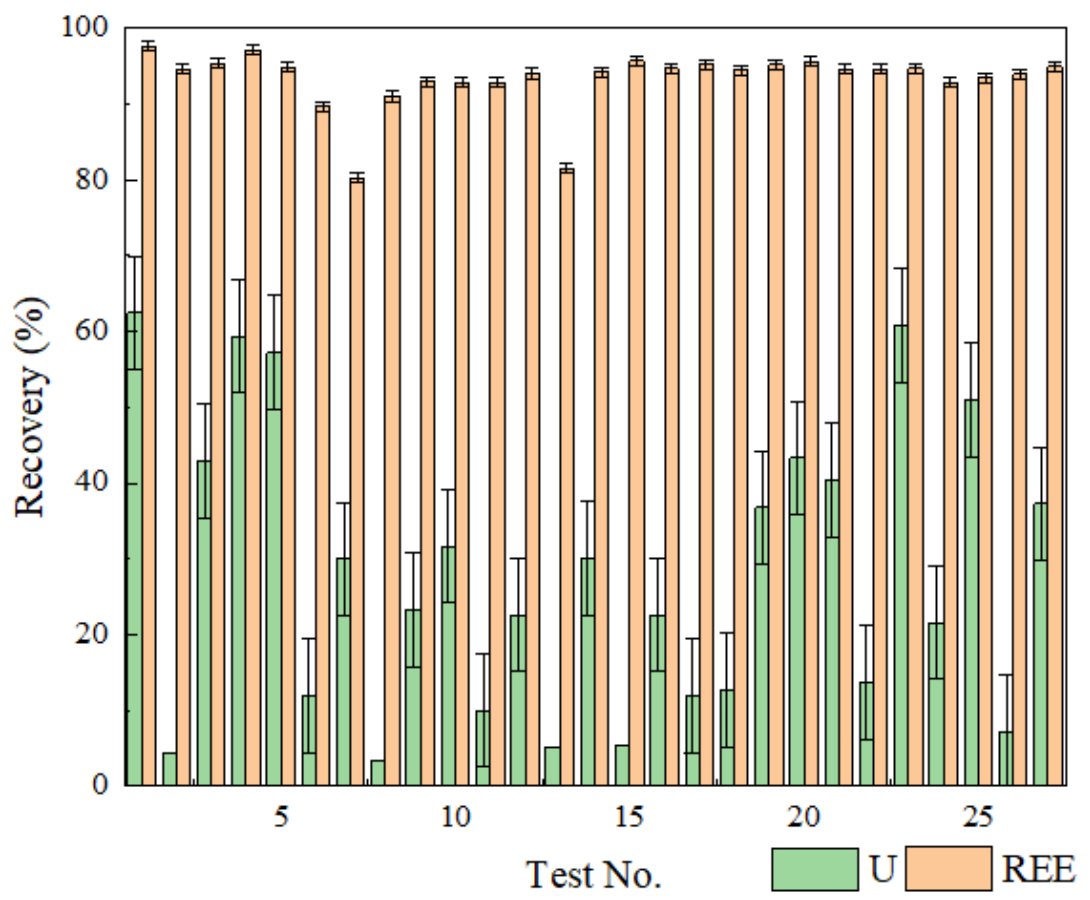

Figure 32 Recovery of total rare earths and uranium into the final rare earth product stream based solely on the solvent extraction circuit. Error bars represent one standard deviation of three replicate tests. 


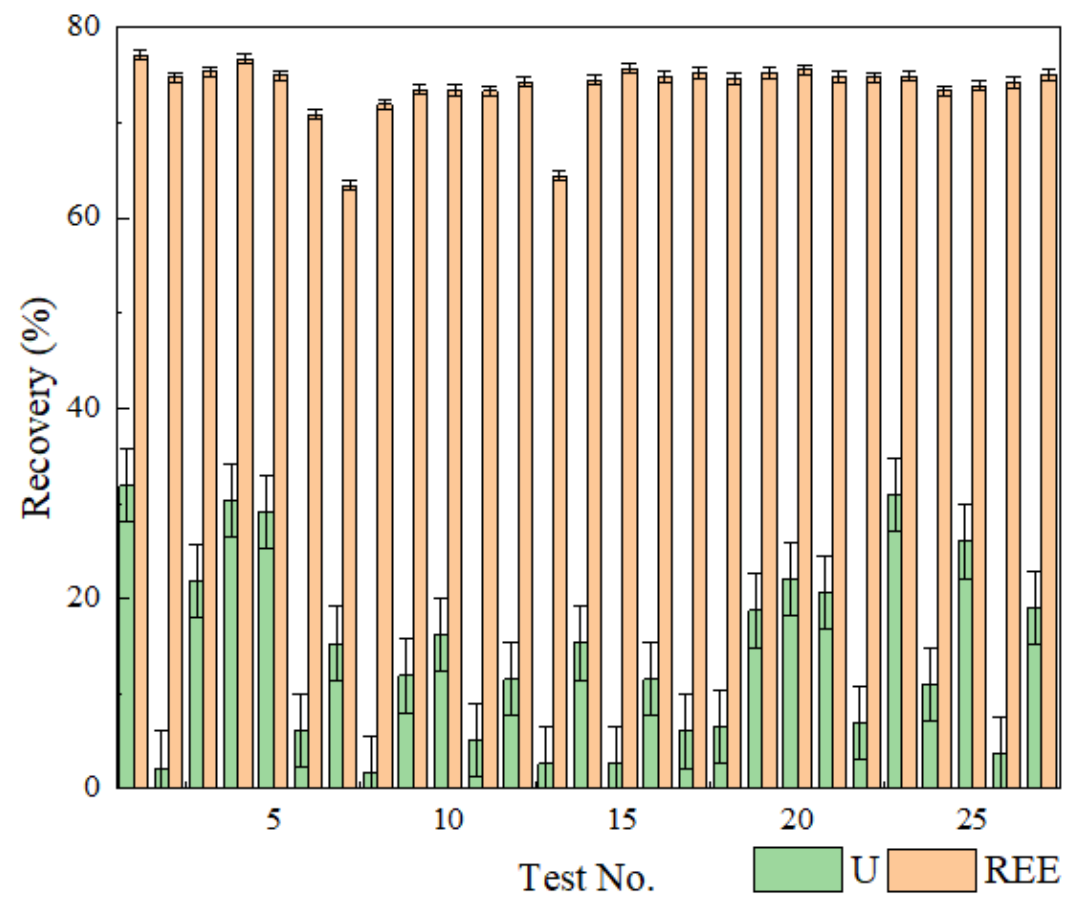

Figure 33 Overall recovery of total rare earths and uranium with respect to the whole experimental flowsheet. Error bars represent one standard deviation of three replicate tests.

During the first set of solvent extraction tests, it was observed that the test conditions that generated the highest rare earth recovery and the lowest uranium recovery were identical. However, the second set presented a different finding. Among all the 27 tests, the lowest uranium recovery (i.e., $3.4 \%$, based solely on solvent extraction) into the rare earth product stream was produced by Test 8 with a corresponding rare earth recovery of $91 \%$. On the other hand, the highest rare earth recovery (i.e., $97.6 \%$, based solely on solvent extraction circuit) into the final product stream was generated by Test 1 . However, the operating conditions of Test 1 resulted in a considerably higher uranium recovery of $62.5 \%$. The elemental composition of the final REE product stream obtained from Test 8 is given as well in Table 18. In the previous set of solvent extraction tests, it was noticed that a portion of uranium was reported to the organic stream. The amount of uranium reporting to the organic stream noticeably decreased in this set of tests which further supports the effect of the stripping agent. For example, for Test 8 , no uranium was recovered into the organic stream on the basis of a solely solvent extraction circuit. Under the same conditions, $3.4 \%$ of uranium reported to the rare earth product stream and the remaining uranium all reported to the uranium product stream, as expected. In addition, the amount of rare earths reporting to the organic stream was negligible $(<1 \%)$ for most of the tests, and the highest recovery of rare earths into the organic stream was seen as $5.9 \%$.

Complexation reactions occurring during extraction and stripping stages are shown in Eqs. (29) (31). The extraction ability of TBP is attributed to the phosphoryl group, which forms solvates with the metal ions in the solution (Giri and Nath, 2016). The extraction of rare earths increases 
proportionally to the atomic number (Peiro and Mendez, 2013). Figure 34 (A) and Figure 34 (B) show the distribution ratio of rare earths and uranium during extraction. Distribution ratios were calculated for total rare earths and uranium during each extraction step. $\mathrm{D}_{1}$ represents the element concentration ratio of organic to aqueous phases during the first extraction, and similarly, $\mathrm{D}_{2}$ shows that of the second extraction. Although the distribution ratio is not necessarily related to the extraction efficiency, it is an indicator of the necessity of multistage extraction. Generally, a decrease in the second distribution ratio was observed for rare earths. This reduction may occur due to the completion of extraction during the first stage. However, there are a few exceptions for which the second step enhanced the extraction of rare earths, as seen in Figure 34 (A). A similar trend was observed for uranium, although this time, the effect of the second extraction step is more profound. The distribution ratio of uranium under the best operating conditions (Test 8) changed from 0.84 to 2 , which resulted in the lowest uranium recovery into the final rare earth product stream. The separation factor between REEs/U is seen in Figure 35. The separation factor is a good measurement of the extractant's separation ability. The data indicate the effect of two-stage extraction on selectively removing uranium from rare earths.
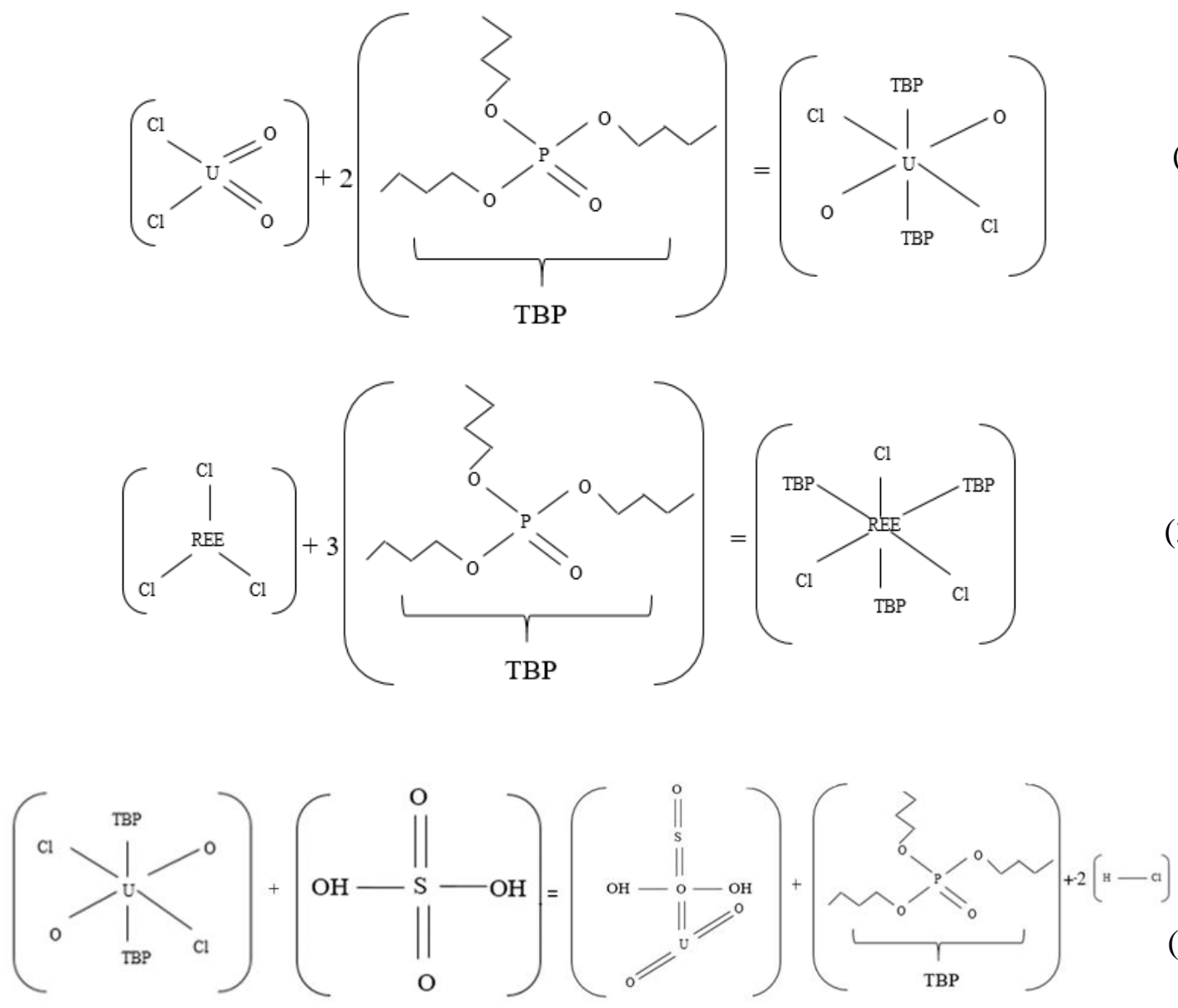
Table 18 Elemental composition of individual rare earth and uranium (ppm) in the final REE product stream obtained from Test 8.

\begin{tabular}{|c|c|c|c|c|c|c|c|c|c|c|c|c|c|c|c|c|}
\hline $\mathrm{Sc}$ & $\mathrm{Y}$ & $\mathrm{La}$ & $\mathrm{Ce}$ & $\operatorname{Pr}$ & $\mathrm{Nd}$ & $\mathrm{Sm}$ & $\mathrm{Eu}$ & $\mathrm{Gd}$ & $\mathrm{Tb}$ & Dy & Ho & $\mathrm{Er}$ & $\mathrm{Tm}$ & $\mathrm{Yb}$ & $\mathrm{Lu}$ & $\mathrm{U}$ \\
\hline 0.00 & 0.08 & 0.05 & 0.37 & 0.06 & 0.24 & 0.08 & 0.01 & 0.05 & 0.00 & 0.02 & 0.00 & 0.01 & 0.00 & 0.00 & 0.00 & 0.00 \\
\hline
\end{tabular}
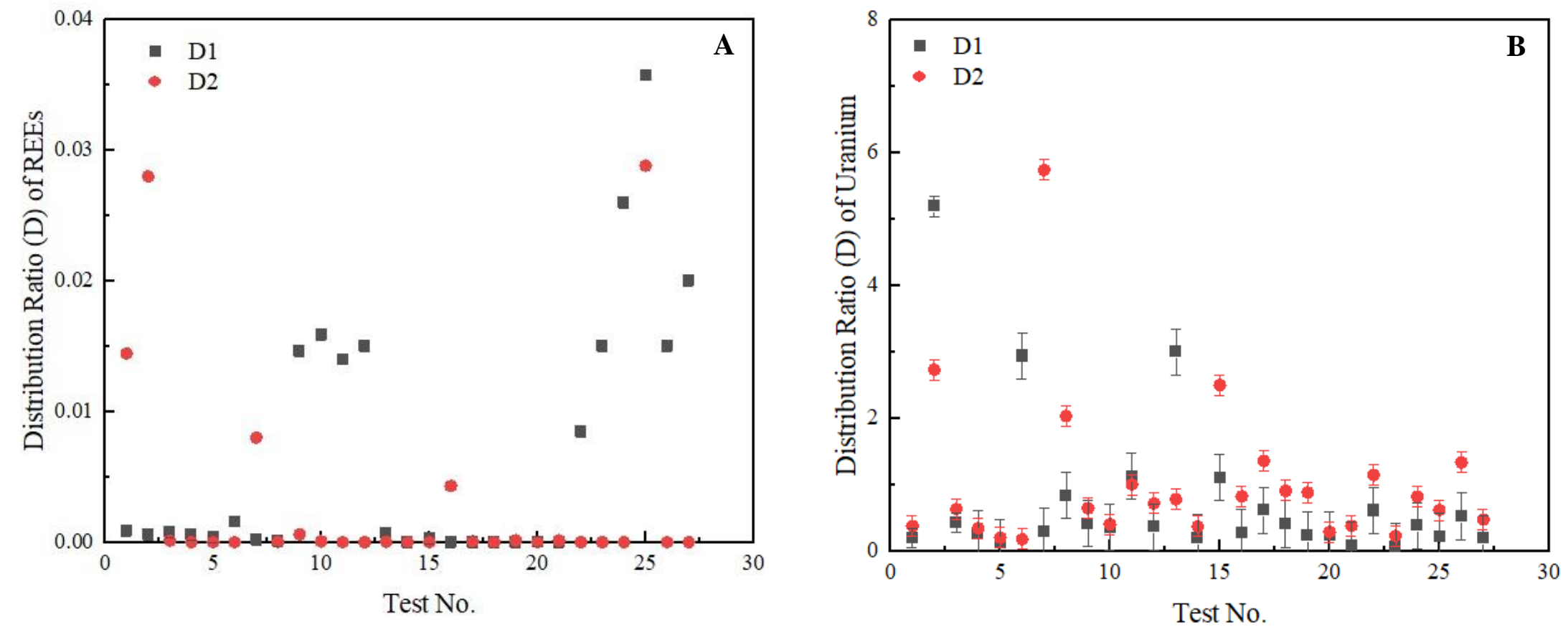

Figure 34 Distribution ratio of (A) total rare earths, (B) uranium in the two extraction stages. Error bars represent one standard deviation of three replicate tests. 


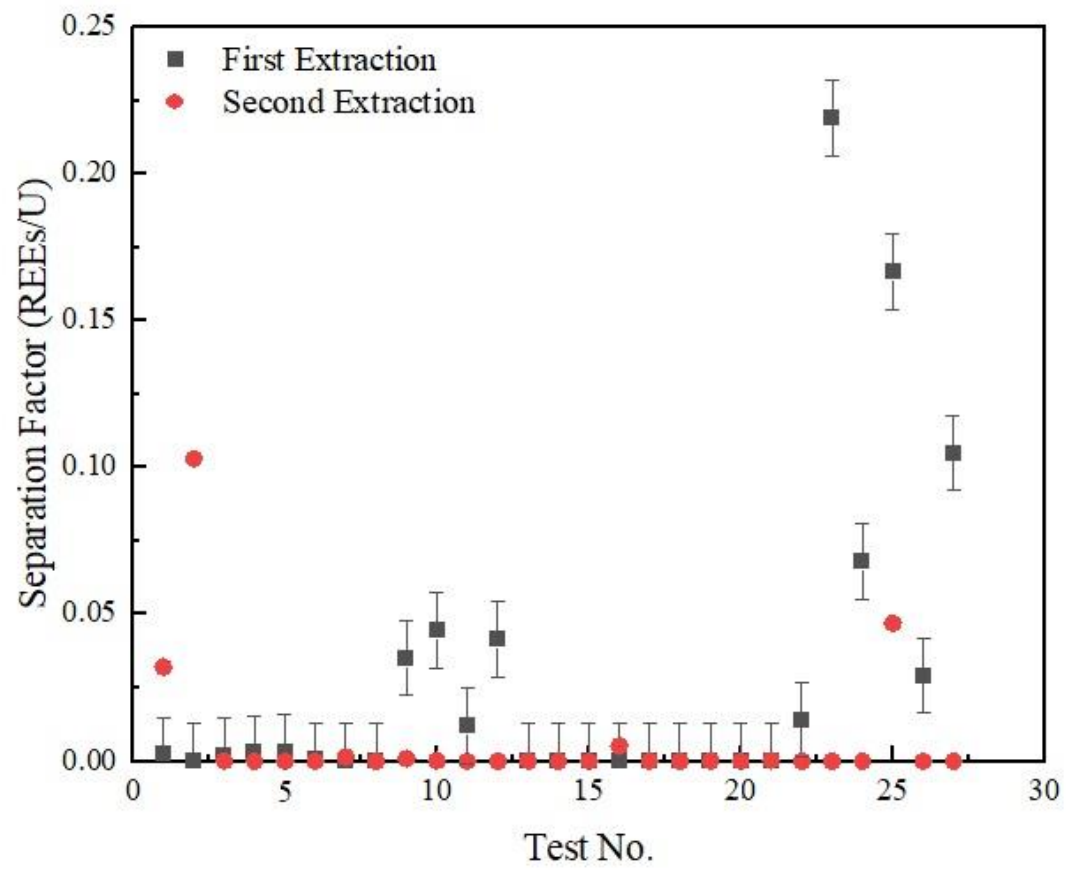

Figure 35 Separation factor between total rare earths and uranium. Error bars represent one standard deviation of three replicate tests.

\subsubsection{Statistical Analysis and Modeling of Second Subset of Solvent Extraction Tests}

The same methodology during the first subset followed, and a statistical model was developed to predict the recovery of uranium by incorporating all the influential operating parameters, as shown in equation 32. It can be seen from the quadratic model that all variables except the stripping agent concentration had a significant impact on the recovery of uranium.

$$
\begin{aligned}
& \text { Uranium Recovery }=+18.64-13.06 * A-15.41 * B+1.99 * C-14.02 * D+4.75 * A B-3.90 * \\
& A C-1.74 * A D+0.54 * B C+7.55 * B D-4.92 * C D+8.24 * A^{2}+2.25 * B^{2}+6.29 * C^{2}+4.54 * \\
& D^{2}
\end{aligned}
$$

where the uncoded model term $\mathrm{A}, \mathrm{B}, \mathrm{C}$, and $\mathrm{D}$ is corresponding to the extractant concentration, feed solution $\mathrm{pH}$, strippant concentration, and $\mathrm{O}$ : A ratio, respectively.

The Analysis of Variance (ANOVA) table of this statistical model is shown in Table 19. The model F-value of 5.90 implies the model is significant. The probability of an F-value to become this large is $0.19 \%$ which is noticeably lower than the critical P-value (5\%). Terms A (extractant concentration), $\mathrm{B}(\mathrm{pH})$, and $\mathrm{D}$ (organic/aqueous ratio) are significant model terms with P-values less than $5 \%$. The same conclusion was reached as a result of the first set of tests. On the other hand, the term D (strippant concentration) has a P-value of 0.5036 outside the confidence level and is considered an insignificant model term due to the same reason. Although the concentration of the strippant tested in the study did not signifcantly impact the recovery of uranium, the data indicate a reduction in the uranium recoveries after using sulfuric acid as the strippant compared 
to deionized water. The concentration of the strippant may not contribute to the improved separation of uranium, but using a different strippant itself (i.e., $\mathrm{H}_{2} \mathrm{SO}_{4}$ ) can explain. The lack-offit value of 1.94 implies a $38.79 \%$ chance that a lack-of-fit F-value can be this high. R-squared values were also given in Table 19 to further evaluate the robustness of the developed model. As seen, an R-square value of 0.8731 and an adjusted R-squared value of 0.7250 confirms the significance of the model. The adequate precision value describes the signal-to-noise ratio, which compares the range of predicted values at the design points to the average prediction error. In general, ratios greater than 4 are desirable and in this case, the adequate precision value is 8.03, which exceeds the desired ratio, and indicates an adequate signal.

Table 19 ANOVA analysis of the quadratic model for the prediction of uranium recovery.

\begin{tabular}{ccccccc}
\hline Source & $\begin{array}{c}\text { Sum of } \\
\text { Squares }\end{array}$ & df & $\begin{array}{c}\text { Mean } \\
\text { Square }\end{array}$ & F Value & $\begin{array}{c}\text { p-value } \\
\text { Prob> F }\end{array}$ & \\
\hline Model & 8250.39 & 14 & 589.31 & 5.90 & 0.0019 & Significant \\
A- Extractant & 2045.46 & 1 & 2045.46 & 20.46 & 0.0007 & \\
B- pH & 2849.62 & 1 & 2849.62 & 28.51 & 0.0002 & \\
C- Strippant & 47.52 & 1 & 47.52 & 0.48 & 0.5036 & \\
D- O/A Ratio & 2359.57 & 1 & 2359.57 & 23.61 & 0.0004 & \\
AB & 90.25 & 1 & 90.25 & 0.90 & 0.3608 & \\
AC & 60.76 & 1 & 60.76 & 0.61 & 0.4507 & \\
AD & 12.18 & 1 & 12.18 & 0.12 & 0.7331 & \\
BC & 1.17 & 1 & 1.17 & 0.012 & 0.9158 & \\
BD & 228.31 & 1 & 228.31 & 2.28 & 0.1566 & \\
CD & 96.73 & 1 & 96.73 & 0.97 & 0.3447 & \\
A2 & 361.72 & 1 & 361.72 & 3.62 & 0.0814 & \\
B2 & 27.10 & 1 & 27.10 & 0.27 & 0.6121 & \\
C2 & 211.29 & 1 & 211.29 & 2.11 & 0.1716 & \\
D2 & 110.07 & 1 & 110.07 & 1.10 & 0.3147 & \\
R-Squared & 0.8731 & & & & & \\
Adj R-Squared & 0.7250 & & & & & \\
Pred R-Squared & 0.3105 & & & & \\
Adeq Precision & 8.029 & & & &
\end{tabular}

Like the first subset, here again, 3D response surface plots were given in Figure 36 to graphically represent the relation between the parameters and their subsequent effect on uranium recovery. It was observed from the experimental test results that an increase in the operational parameters produces a lower uranium recovery. However, when the interacting effect of the model terms was further analyzed, it was seen that any reduction in the uranium recovery into the final product stream does not depend solely on a single parameter. A simultaneous increase in the extractant concentration, $\mathrm{O} / \mathrm{A}$ ratio, and solution $\mathrm{pH}$ generates the best separation performance. Figure 36(A) shows the effect of extractant concentration and $\mathrm{pH}$ on uranium recovery while the O/A ratio and stripping agent concentration were fixed at 2 and $1 \mathrm{~mol} / \mathrm{L}$, respectively. One of the highest uranium 
recovery $(57.22 \%)$ of this set of tests was observed when the feed solution $\mathrm{pH}$ and extractant concentrations were 2.5 and $30 \%$ by volume, respectively. A gradual increase in the $\mathrm{pH}$ and extractant concentration lowered the uranium recovery. The lowest uranium recovery (3.42\%) into the rare earth product stream was obtained at a $\mathrm{pH}$ value of 3.5 with $50 \%$ TBP by volume. These findings prove that the $\mathrm{pH}$ of the feed solution and the extractant concentration are the two critical factors impacting uranium recovery. A higher extractant concentration enhanced the process, and higher uranium removal from the aqueous phase to the organic phase was achieved. The same conclusion was reached in the previous set of tests with the lowest uranium recovery being obtained under identical conditions (i.e., pH of 3.5 and $50 \mathrm{v} \%$ TBP). Figure 36(B) shows the effect of extractant concentration and stripping agent concentration while the feed solution $\mathrm{pH}$ and $\mathrm{O} / \mathrm{A}$ ratio were kept constant at 3.5 and 2 , respectively. It can be seen that changing the stripping agent from DI water to sulfuric acid had an impact on uranium recovery. In both sets of the tests, the lowest uranium recovery was obtained under the same conditions (i.e., $50 \mathrm{v} \% \mathrm{TBP}$ at pH 3.5 with $\mathrm{O} / \mathrm{A}$ ratio 3 ), with the only difference being the stripping agent. The uranium recovery was reduced by approximately $2.5 \%$ after switching the strippant, which indicates the effect of sulfuric acid to some extent. However, the concentration of sulfuric acid tested in the study did not generate a significant impact on the separation results. In Figure $36(\mathrm{C})$, the effect of the O/A ratio and extractant concentration on uranium recovery can be seen. The uranium recovery into the final product stream decreases with an increase in both extractant concentration and O/A ratio. The highest uranium recovery of $62.5 \%$ was achieved with the lowest extractant concentration $(30 \%$ $\mathrm{TBP}$ ) and $\mathrm{O} / \mathrm{A}$ ratio of 1 . A low $\mathrm{O} / \mathrm{A}$ ratio may not be sufficient to extract uranium ions from the aqueous phase for separation the purpose. Therefore, an increase in the O/A ratio results in an improved separation. Additionally, a high $\mathrm{O} / \mathrm{A}$ ratio and $\mathrm{pH}$ produce noticeably enhanced separation results. Similar findings were also obtained in Jorjani and Shabazi's study (2012). Figure 36(D) further shows the effect of feed $\mathrm{pH}$ value and stripping agent concentration while extractant concentration and $\mathrm{O} / \mathrm{A}$ ratio were kept constant at $40 \%$ and 2, respectively. As it was indicated and statistically supported by the model, $\mathrm{pH}$ is an influential parameter, and the best result was obtained at $\mathrm{pH} 3.5$. The lowest uranium recovery was generated using $1 \mathrm{~mol} / \mathrm{L}$ sulfuric acid. Even though an increase in the concentration of stripping agent from $0.5 \mathrm{~mol} / \mathrm{L}$ to $1 \mathrm{~mol} / \mathrm{L}$ lowered the uranium recovery, a further boost from $1 \mathrm{~mol} / \mathrm{L}$ to $2 \mathrm{~mol} / \mathrm{L}$ had a minimum impact on uranium recovery. This indicates that $1 \mathrm{~mol} / \mathrm{L} \mathrm{H}_{2} \mathrm{SO}_{4}$ solution was sufficient to break the uraniumTBP complex and strip uranium back into the aqueous phase. Figure 36(E) displays the effect of $\mathrm{O} / \mathrm{A}$ ratio and solution $\mathrm{pH}$ and it can be seen from the figure that a high $\mathrm{O} / \mathrm{A}$ ratio and $\mathrm{pH}$ produce noticeably enhanced separation results. Moreover, a lower uranium recovery observed with an increase in the solution $\mathrm{pH}$ suggests that the higher $\mathrm{pH}$ favors creating more stable uranium-TBP complexes. Lastly, the plot in Figure 36(F) shows the effect of the O/A ratio and stripping agent concentration on uranium recovery while keeping the extractant concentration and $\mathrm{pH}$ constant at $40 \%$ and 3.5 , respectively.

Similar to the first subset, 2D contour plots were generated for this subset to further evaluate the degree of interaction between the variables tested during the experiments. Elliptical contours 
observed for Figure 37(A), (D), and (E) indicate strong interaction, whereas Figure 37(B) and Figure 37(F) represent relatively less significant interaction. Among all the contour plots, Figure 37(C) displays the smallest interaction with more circular lines.

Statistical analysis was also performed for the recovery of total rare earths, and a model was developed. Although a model was obtained, the ANOVA indicates that the model is not statistically meaningful. However, the insignificance of this model is consistent with the experimental findings of both sets of tests. Considering the objective of this experimental design is to investigate the effect of different input operating variables on uranium recovery as opposed to rare earths recovery. Therefore, it is not surprising that the input variables had a minimum impact on the recovery of rare earths compared to uranium. This finding confirms the selectivity of the experimental design.
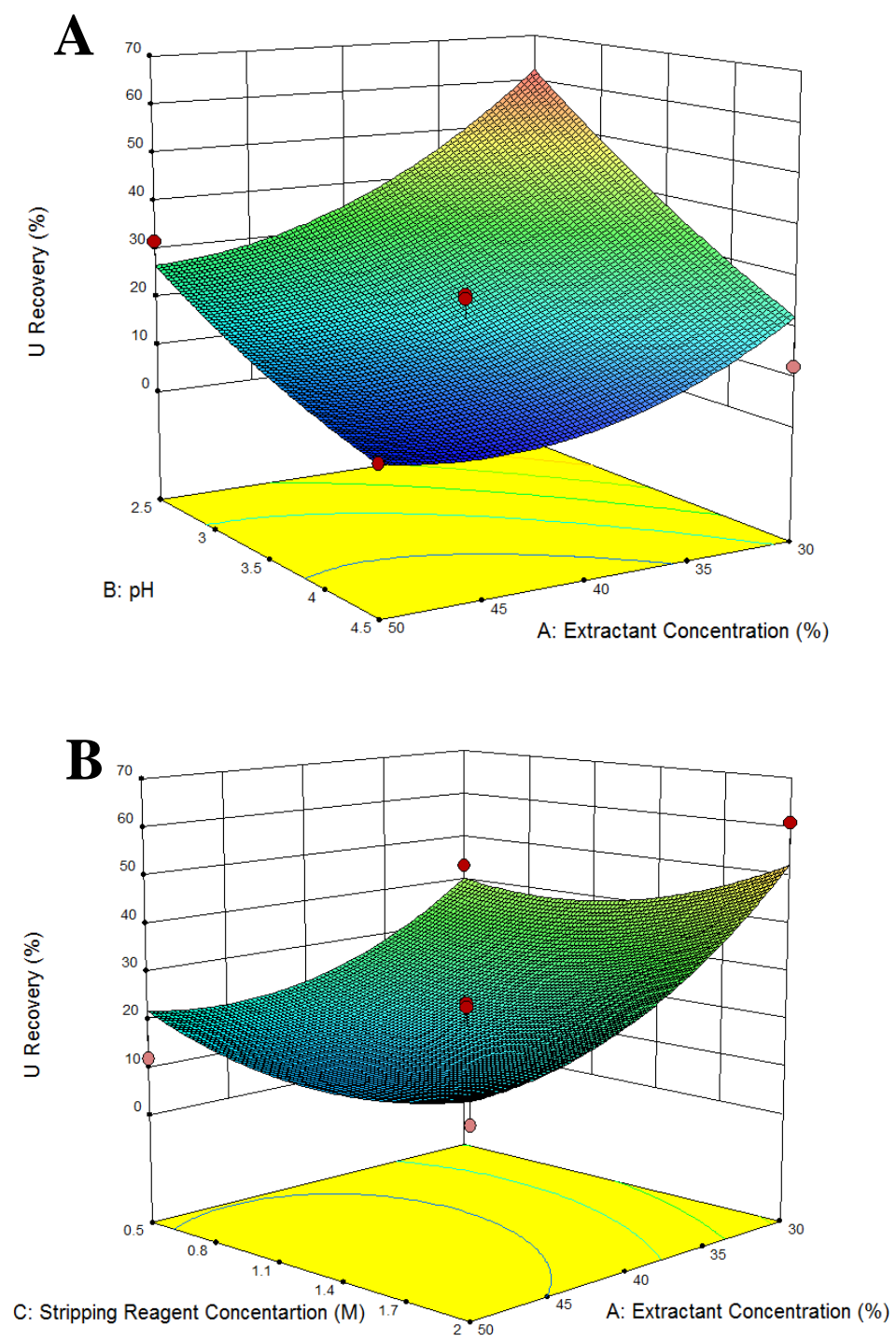

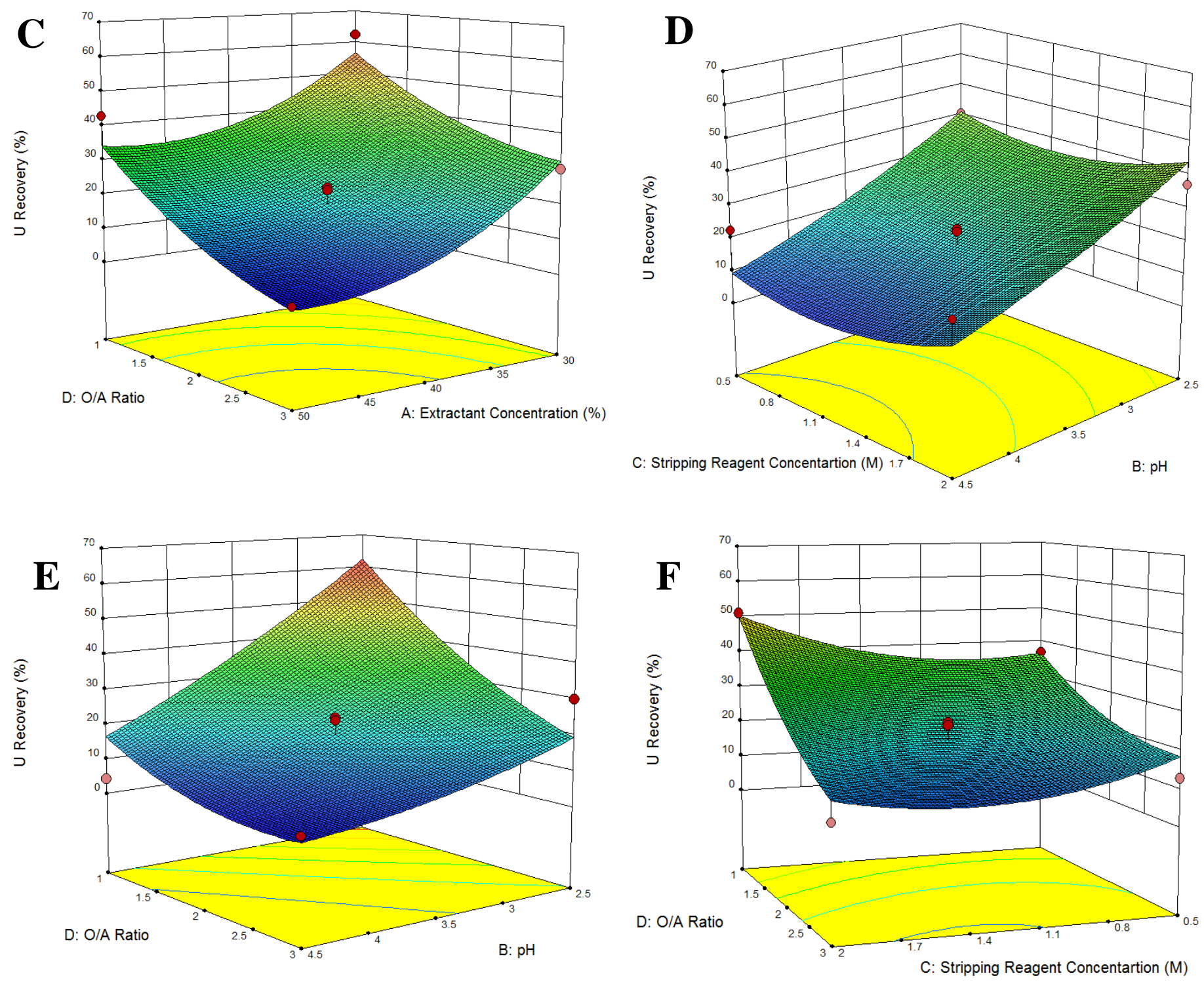

Figure 36 3D surface response graphs obtained for the statistical model predicting uranium recovery. 

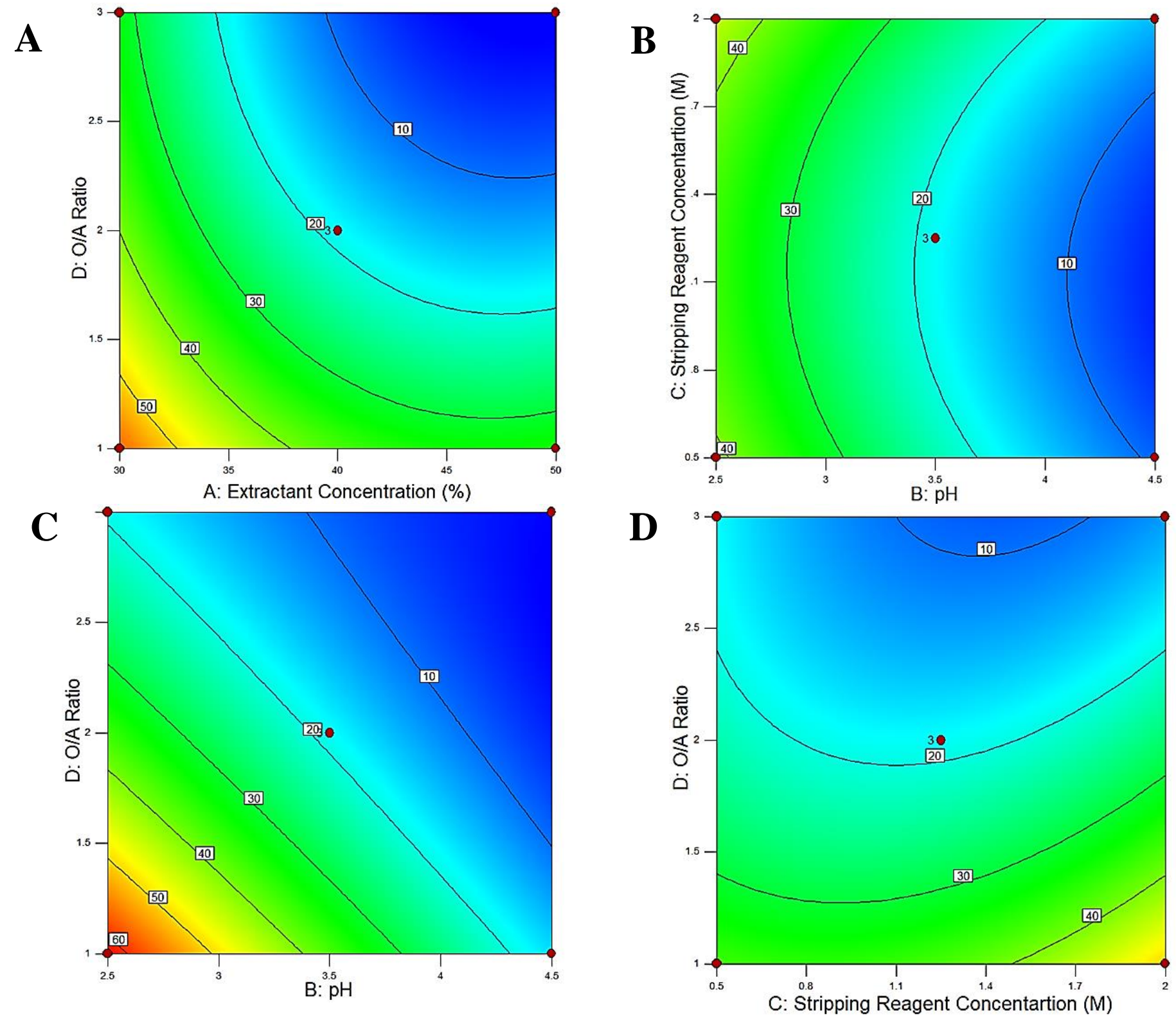

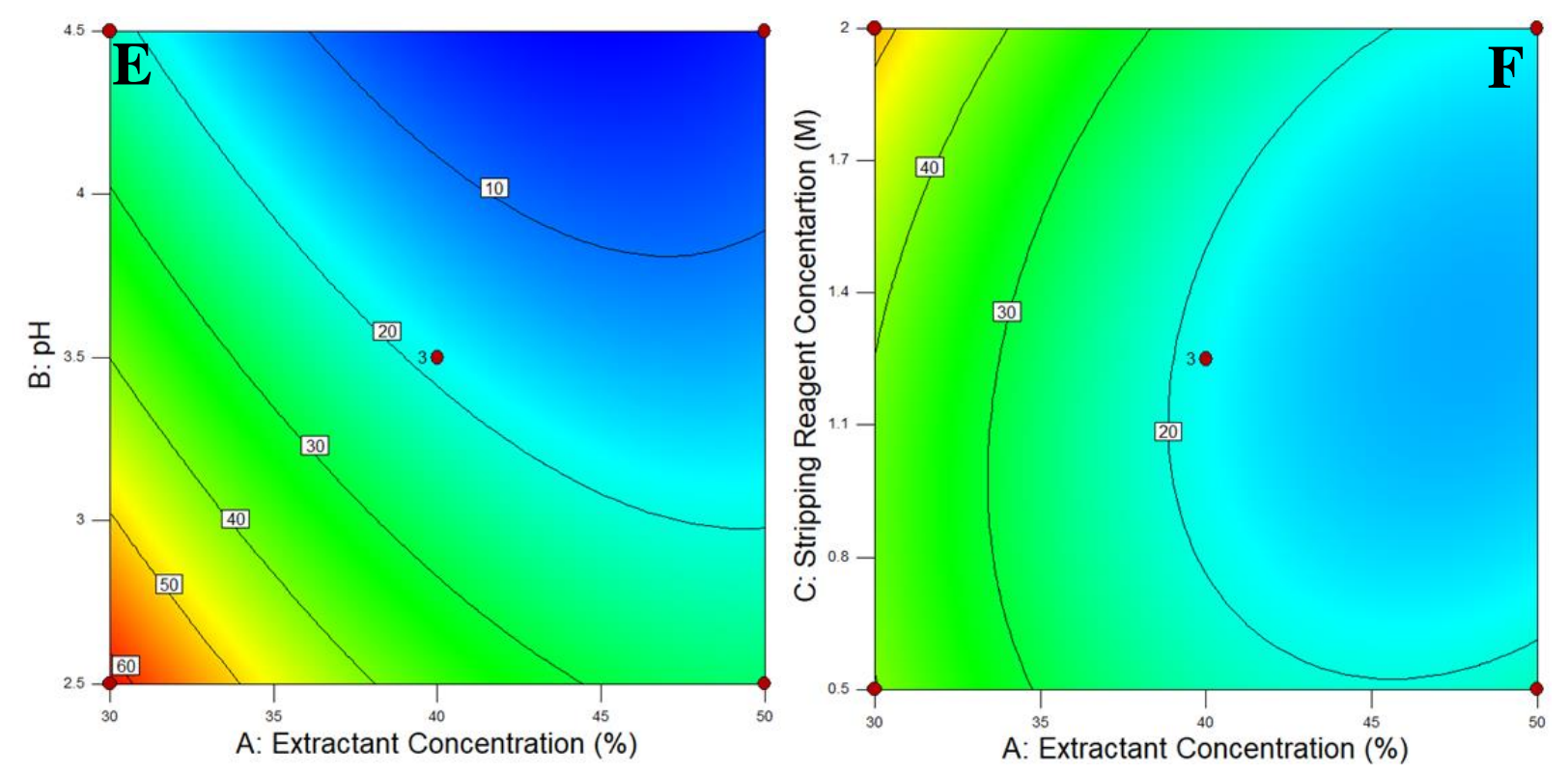

Figure 37 2D contour plots obtained for the statistical model predicting uranium recovery.

\subsection{Rare Earth Oxalate Studies}

A new set of tests were conducted with the rare earth oxalate sample provided by the University of Kentucky. This sample was generated at the pilot processing plant operated by the project partner researchers, and it was used as a validation of previously obtained test results. The elemental composition of the rare earth oxalate sample is given in Table 20, and the process flowsheet followed for thorium removal is given in Figure 38. As seen, the total rare earths concentration is around $35 \%$, and the light rare earths constitute the majority. While there was no uranium detected, thorium content is $0.12 \mathrm{mg} / \mathrm{g}$.

Table 20 Elemental composition of the rare earth oxalate sample.

\begin{tabular}{ccccccccc}
\hline Element & $\mathrm{Sc}$ & $\mathrm{Y}$ & $\mathrm{La}$ & $\mathrm{Ce}$ & $\mathrm{Pr}$ & $\mathrm{Nd}$ & $\mathrm{Sm}$ & $\mathrm{Eu}$ \\
\hline Concentration $(\mathrm{mg} / \mathrm{g})$ & 0.14 & 14.42 & 66.20 & 144.80 & 17.78 & 72.42 & 12.46 & 2.20 \\
\hline Element & $\mathrm{Gd}$ & $\mathrm{Tb}$ & $\mathrm{Dy}$ & $\mathrm{Ho}$ & $\mathrm{Er}$ & $\mathrm{Tm}$ & $\mathrm{Yb}$ & $\mathrm{Lu}$ \\
\hline Concentration $(\mathrm{mg} / \mathrm{g})$ & 8.56 & 0.83 & 3.35 & 0.56 & 1.32 & 0.14 & 0.76 & 0.10 \\
\hline Element & $\mathrm{Th}$ & $\mathrm{Ca}$ & $\mathrm{Fe}$ & & & & & \\
\hline Concentration $(\mathrm{mg} / \mathrm{g})$ & 0.12 & 30.75 & 9.31 & & & & & \\
\hline
\end{tabular}

Initially, a $10 \mathrm{~g}$ rare earth oxalate sample was roasted at $600{ }^{\circ} \mathrm{C}$ for 2 hours to produce rare earth oxide. After roasting, $5.7 \mathrm{~g}$ of solid was obtained. After taking a representative sample for elemental analysis, the remaining solid sample was washed with $0.5 \mathrm{~mol} / \mathrm{L} \mathrm{HCl}$ acid to remove calcium. During the calcium wash step, the agitation was provided by a magnetic stirrer at approximately $525 \mathrm{rpm}$. After 15 minutes of washing, the solution was filtered using $0.45 \mu \mathrm{m}$ pore size filter paper, and the solid that remained on filter paper was dried in the oven overnight. Solid, 
obtained after the calcium removal step, was weighed, and a sample was taken for analysis. The sample was taken from the wash effluent as well.

Following the calcium removal step, leaching was performed at a concentration of $0.5 \mathrm{~g} / \mathrm{L}$ using 6 $\mathrm{mol} / \mathrm{L}$ hydrochloric $(\mathrm{HCl})$ acid at $80{ }^{\circ} \mathrm{C}$. Mixing was continued for 24 hours. After leachate was generated, precipitation tests were conducted. $50 \%$ w/w $\mathrm{NaOH}$ was used to adjust the $\mathrm{pH}$. When the targeted $\mathrm{pH}$ value was achieved, the test was stopped, the solution was centrifuged for $15 \mathrm{~min}$, and then it was subjected to filtration using $0.45 \mu \mathrm{m}$ pore size filter paper. The sample was taken from the filtrate, and the precipitated solid sample was dried in the oven overnight. All the stagewise precipitation tests were conducted at room temperature, and DI water was used at all steps.

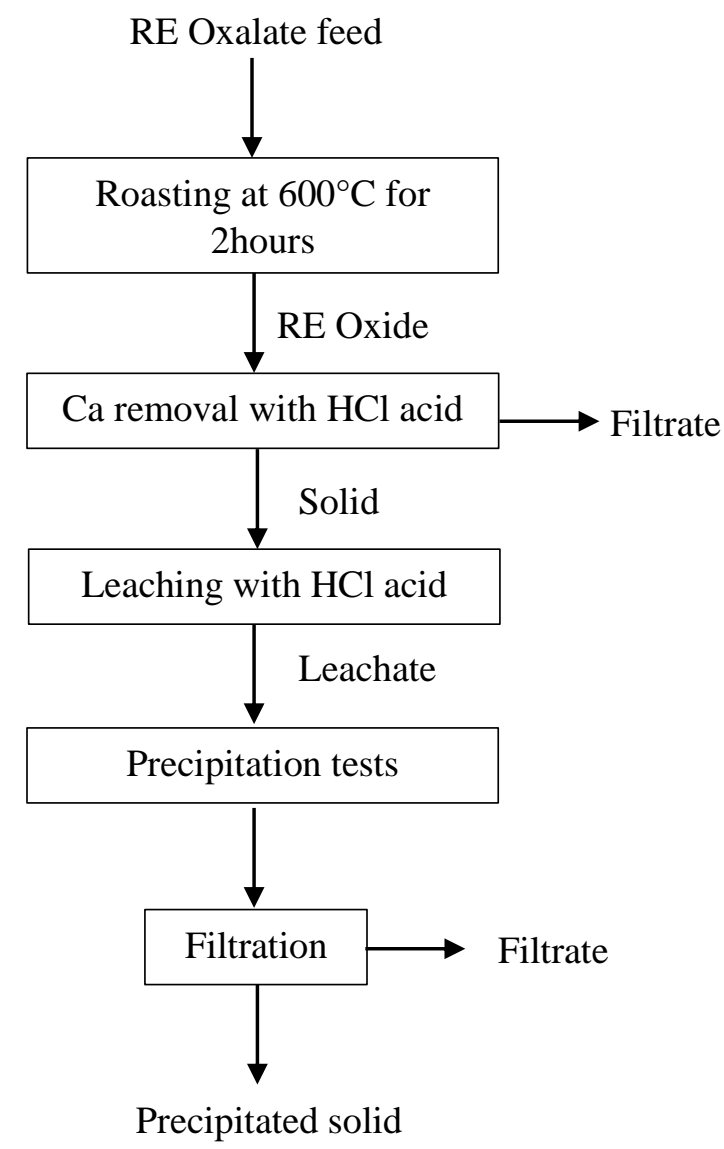

Figure 38 Experimental flowsheet followed for rare earth oxalate sample.

The results obtained from stage-wise precipitation tests are given in Figure 39. The first color change and formation of particles were observed around $\mathrm{pH} 3.5$ for all the tests. However, even after 45 minutes of settling and an additional 20 minutes of centrifuging, nothing was completely precipitated or settled. Therefore, it is not suggested to pause at this $\mathrm{pH}$ value. The results are consistent with the previous selective precipitation tests conducted using the synthetic solution. A good selectivity was observed between thorium and rare earth elements suggesting a $\mathrm{pH}$ value lower than 5. A continuous increase in the precipitation of rare earths was observed, and it reached 
completion at $\mathrm{pH}$ 9.5. A major increase in the precipitation of rare earths was seen between $\mathrm{pH} 7.5$ and 8 , which accounts for $37 \%$.

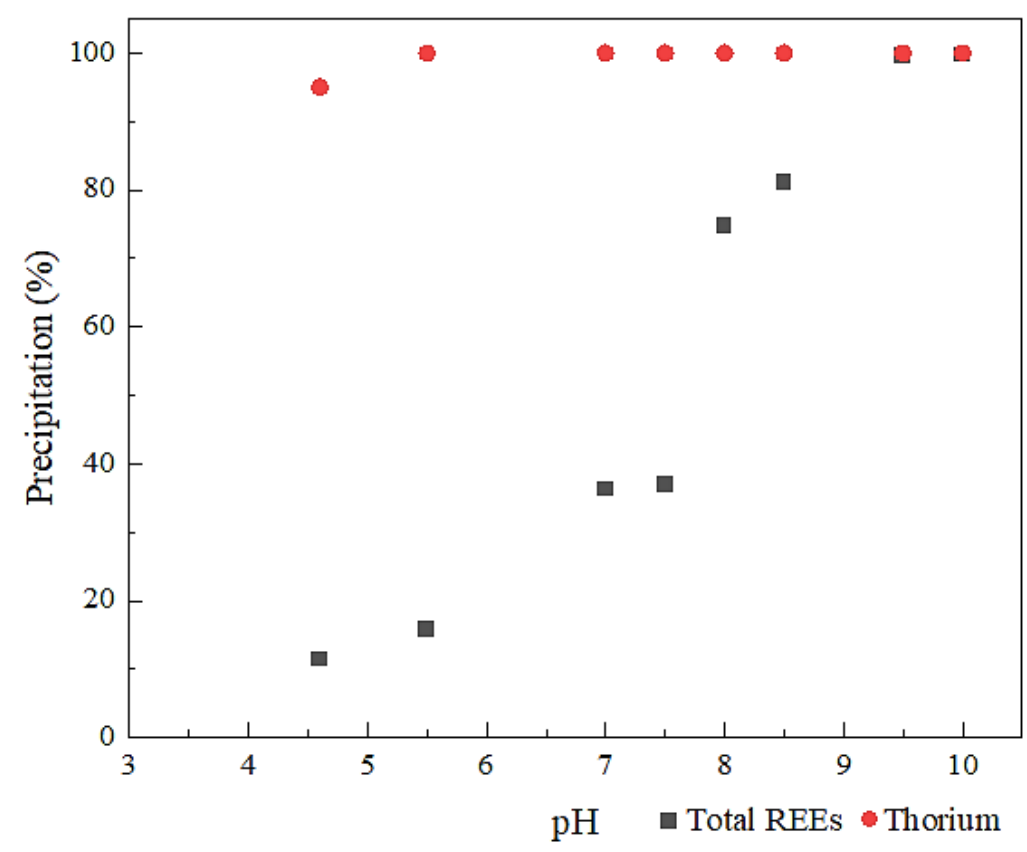

Figure 39 Precipitation of total rare earths and thorium at various $\mathrm{pH}$ values. Error bars represent one standard deviation.

The SEM images of the rare earth oxide sample used throughout the performance validation tests are given in Figure 40. As seen, the morphology is rough and heterogeneous. Also, the sample consists of various irregular-shaped particles. Besides, the presence of relatively big, rectangular prism-shaped particles is noticed. On the contrary, the SEM images (Figure 41) of solids obtained during stage-wise precipitation tests indicate that they have a very smooth surface with clearly identified cubic-shaped particles.
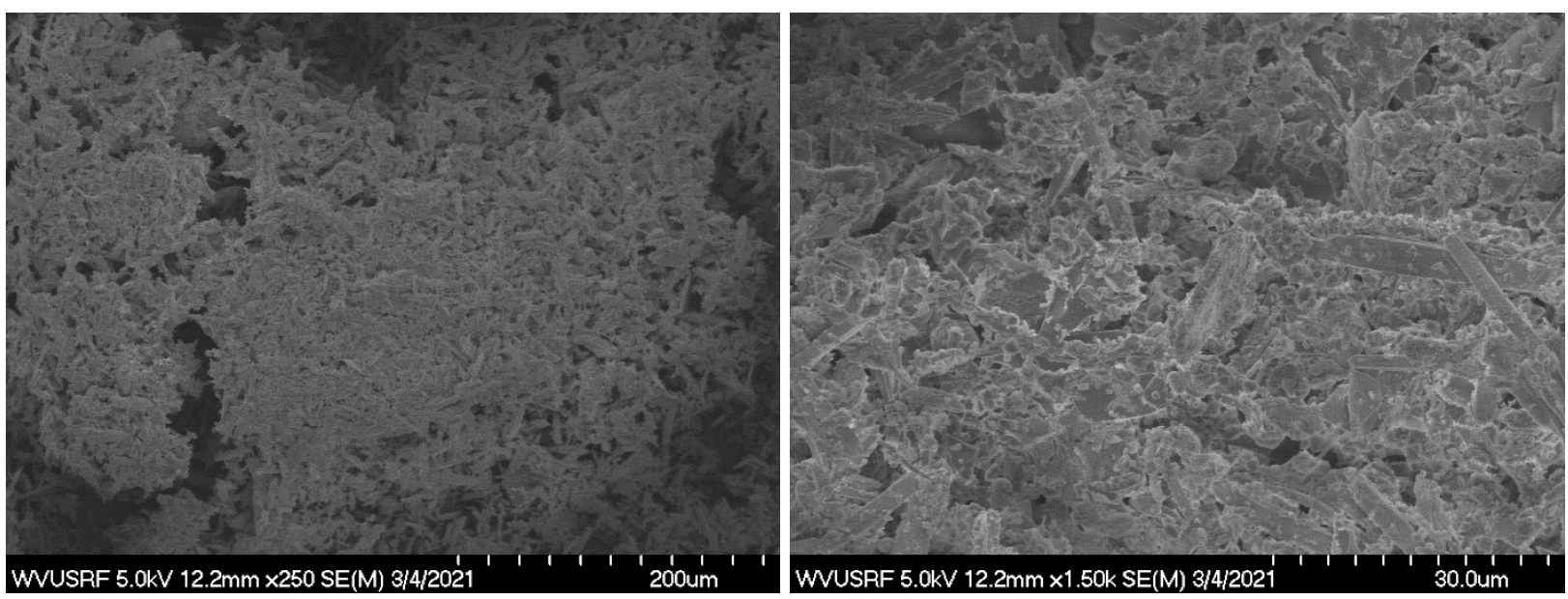

Figure 40 The SEM image of the rare earth oxide feed sample. 

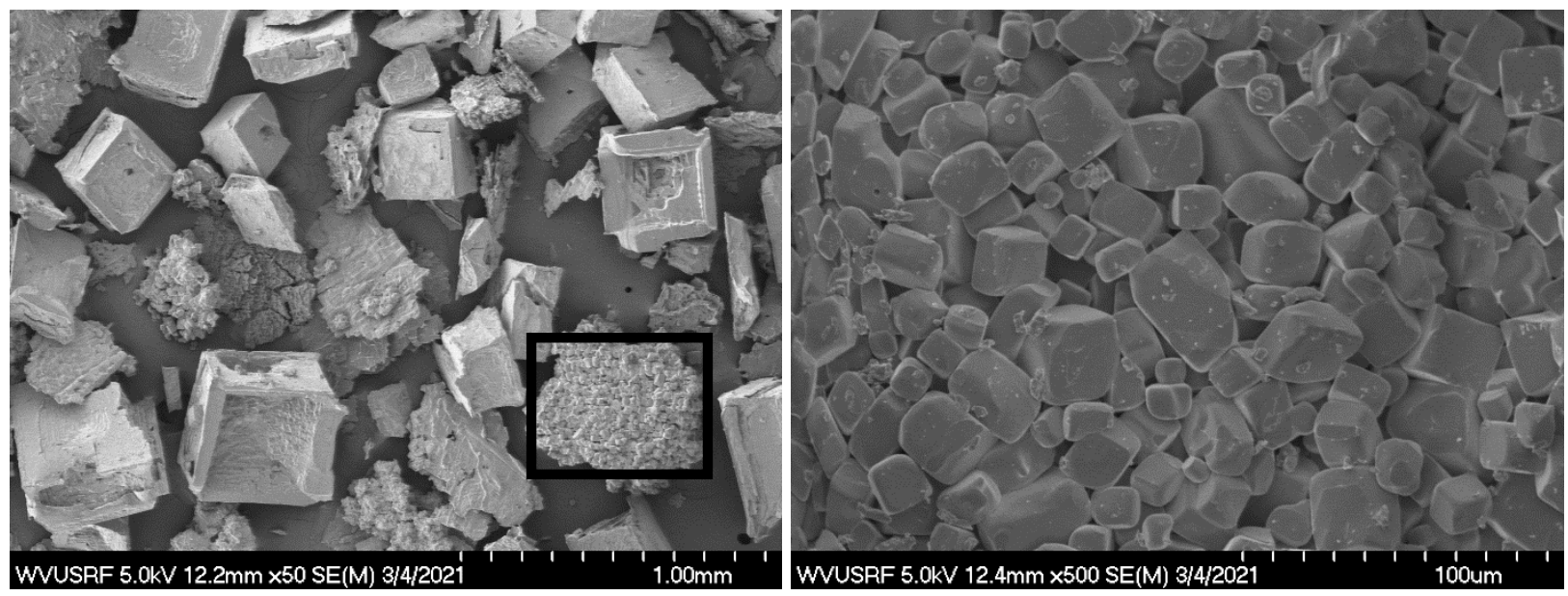

Figure 41 The SEM image of precipitated solids at $\mathrm{pH} 4.6$.

\subsection{Conclusions}

In this chapter, selective precipitation and solvent extraction were applied to assess the separation of thorium and uranium from a rare earth-containing solution. Based on the findings of initial testing, an experimental approach consisting of selective precipitation followed by solvent extraction was developed and strictly followed. Later, the findings were validated using a real system rare earth oxalate sample generated at the pilot processing plant located in Kentucky. Specific findings from this part of the study include:

1. Selective precipitation was an effective technique for the removal of thorium. Nearly $100 \%$ of thorium precipitated out at a solution $\mathrm{pH}$ value of 4.8 , while $19.3 \%$ of rare earths and $47.9 \%$ of uranium co- precipitated at the same $\mathrm{pH}$. A further increase in the solution $\mathrm{pH}$ led to the co-precipitation of rare earths and uranium due to their overlapping precipitation $\mathrm{pH}$ regions.

2. At the same $\mathrm{pH}$ value of 4.8 , approximately $65.6 \%$ of iron precipitated out, followed by around $38.5 \%$ of aluminum, $17.1 \%$ of calcium, and $14.2 \%$ of magnesium precipitation.

3. Species distribution diagrams of thorium, uranium, selected REEs, iron, and aluminum in chloride media corresponded well with the precipitation data generated during selective precipitation, which enhanced the understanding of metal precipitation from a fundamental perspective.

4. The precipitation reactions were spontaneous and exothermic for the elements studied based on calculated Gibbs energy and enthalpy values. All precipitation reactions were thermodynamically favorable at the standard state operating conditions.

5. Solvent extraction preferentially extracted uranium compared to thorium from the REEcontaining solution. A two-stage solvent extraction process proved to be more efficient than the single-stage process, during the former process of which approximately $97 \%, 94 \%$, 
and $43 \%$ of REEs, thorium, and uranium were recovered into the circuit product stream, respectively.

6. Test results following the first subset of the modified experimental protocol indicate that extractant concentration, solvent extraction feed $\mathrm{pH}$, and $\mathrm{O} / \mathrm{A}$ ratio during extraction, all played a significant role in the removal of uranium from rare earths, which the statistical model further corroborated developed using Box-Behnken.

7. The optimum separation performance was identified throughout the study, which corresponded to overall rare earth, thorium, and uranium recovery of $79.6 \%, 0 \%$, and $3.1 \%$, separately. The optimum separation was obtained under the following operating condition: one-stage precipitation at a $\mathrm{pH}$ value of 4.8 , solvent extraction feed $\mathrm{pH}$ of $3.5,50 \mathrm{v} \% \mathrm{TBP}$, $\mathrm{O} / \mathrm{A}$ ratio at 3:1.

8. During the second subset, the recovery of rare earths into the REE product stream varied from $80.2 \mathrm{wt} \%$ to $97.6 \mathrm{wt} \%$ based solely on solvent extraction. In comparison, uranium recovery into the same product steam fluctuated between $3.4 \mathrm{wt} \%$ and $62.5 \mathrm{wt} \%$ under all tested conditions.

9. The lowest overall uranium recovery of $1.8 \mathrm{wt} \%$ into the final product stream was achieved with $50 \mathrm{v} \% \mathrm{TBP}$, feed $\mathrm{pH}$ at 3.5, O/A ratio at 3, and $1 \mathrm{~mol} / \mathrm{L} \mathrm{H}_{2} \mathrm{SO}_{4}$ strippant, which corresponded to an overall rare earth recovery of $73.4 \mathrm{wt} \%$.

10. A statistically significant model was developed for uranium recovery prediction in solvent extraction, which indicates the extractant concentration, solution $\mathrm{pH}$, and $\mathrm{O} / \mathrm{A}$ ratio all played a critical role. On the contrary, the concentration of the stripping agent $\left(\mathrm{H}_{2} \mathrm{SO}_{4}\right)$ was found as an insignificant parameter over the tested concentration range. 


\section{CHAPTER 5}

\section{ZEOLITE ADSORPTION STUDIES}

\subsection{Characterization of Initial Zeolite Samples}

Three zeolite samples with distinctly different size distributions were used to investigate the effect of particle size on the adsorption efficiency. The particle size distribution of these zeolite samples is provided in Figure 42. Figure 42(A) shows the particle size distribution of the coarsest zeolite sample with a $\mathrm{P}_{80}$ passing value of $1490 \mu \mathrm{m}$, whereas Figure 42(B) displays the particle size distribution of the middle-sized zeolite sample with a $\mathrm{P}_{80}$ value of approximately $200 \mu \mathrm{m}$. Lastly, the particle size distribution of the finest sample is provided in Figure 42(C). As seen, a $\mathrm{P}_{80}$ value of approximately $12 \mu \mathrm{m}$ was found for this particular sample. Due to the limit of the particle size analyzer's capability, the particle size distribution of the coarsest zeolite sample was determined via sieve analysis, while the rest two samples were subjected to the laser particle size analysis.
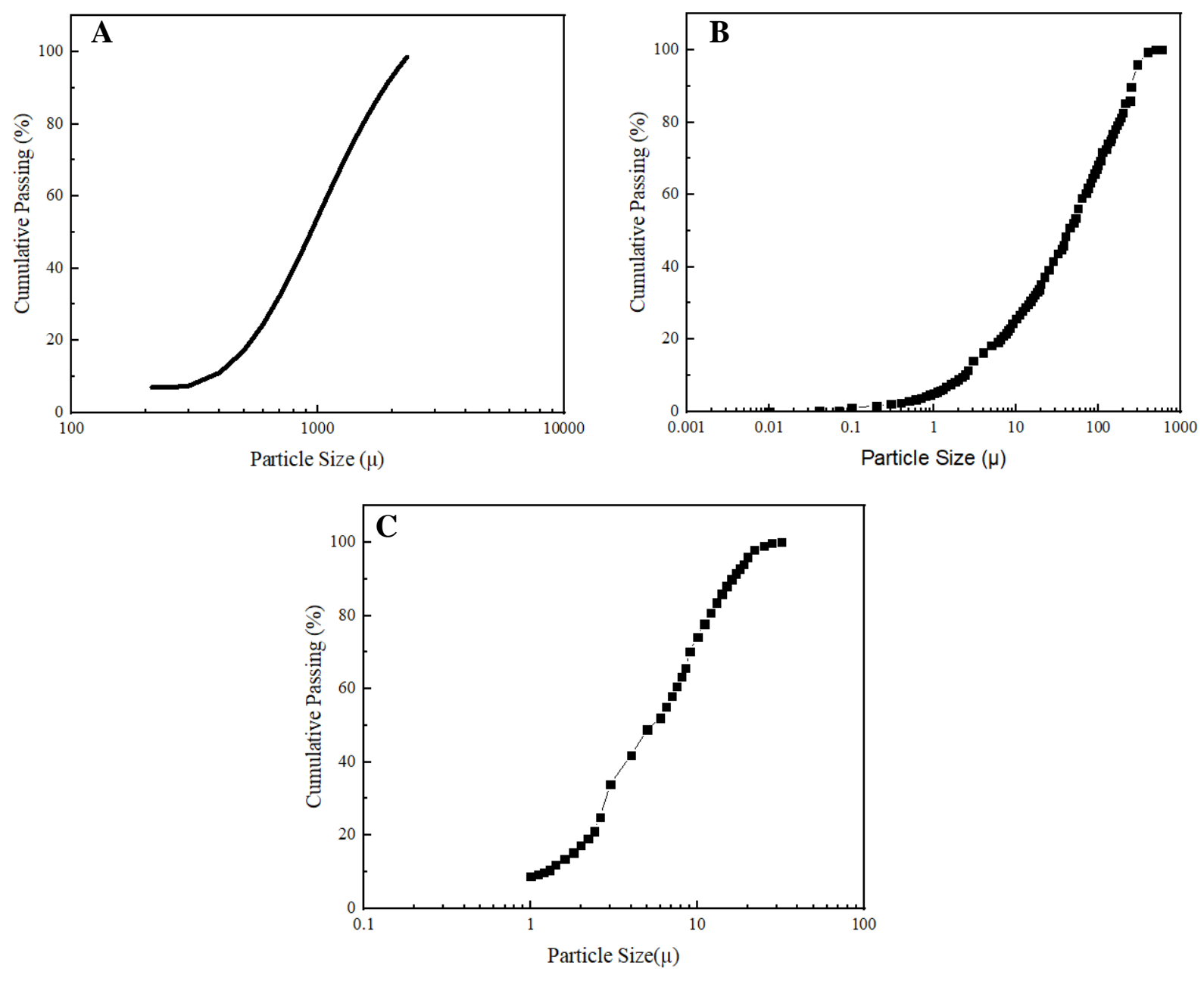

Figure 42 Particle size distribution of three zeolite samples. (A) Coarsest sample, P80=1490 $\mu \mathrm{m}$, (B) Middle-sized sample, P80=200 $\mu \mathrm{m}$, (C) Finest sample, P80=12 $\mu \mathrm{m}$. 
Mineralogical compositions of the samples were identified using XRD. The combined XRD patterns of the three samples are provided in Figure 43. All samples displayed the same diffraction pattern with the most intense peak being at approximately $22^{\circ}$, which refers to the single phase of clinoptilolite-type zeolite. It is the most commonly found zeolite type with the aluminum-silicate tetrahedral arrangement and Si/Al>4 (Godelitsas and Armbruster, 2003). This type of zeolite series has the complex chemical formula of $\mathrm{Na}_{6}\left[\mathrm{Al}_{6} \mathrm{Si}_{30} \mathrm{O}_{72}\right]_{24} \mathrm{H}_{2} \mathrm{O}$.

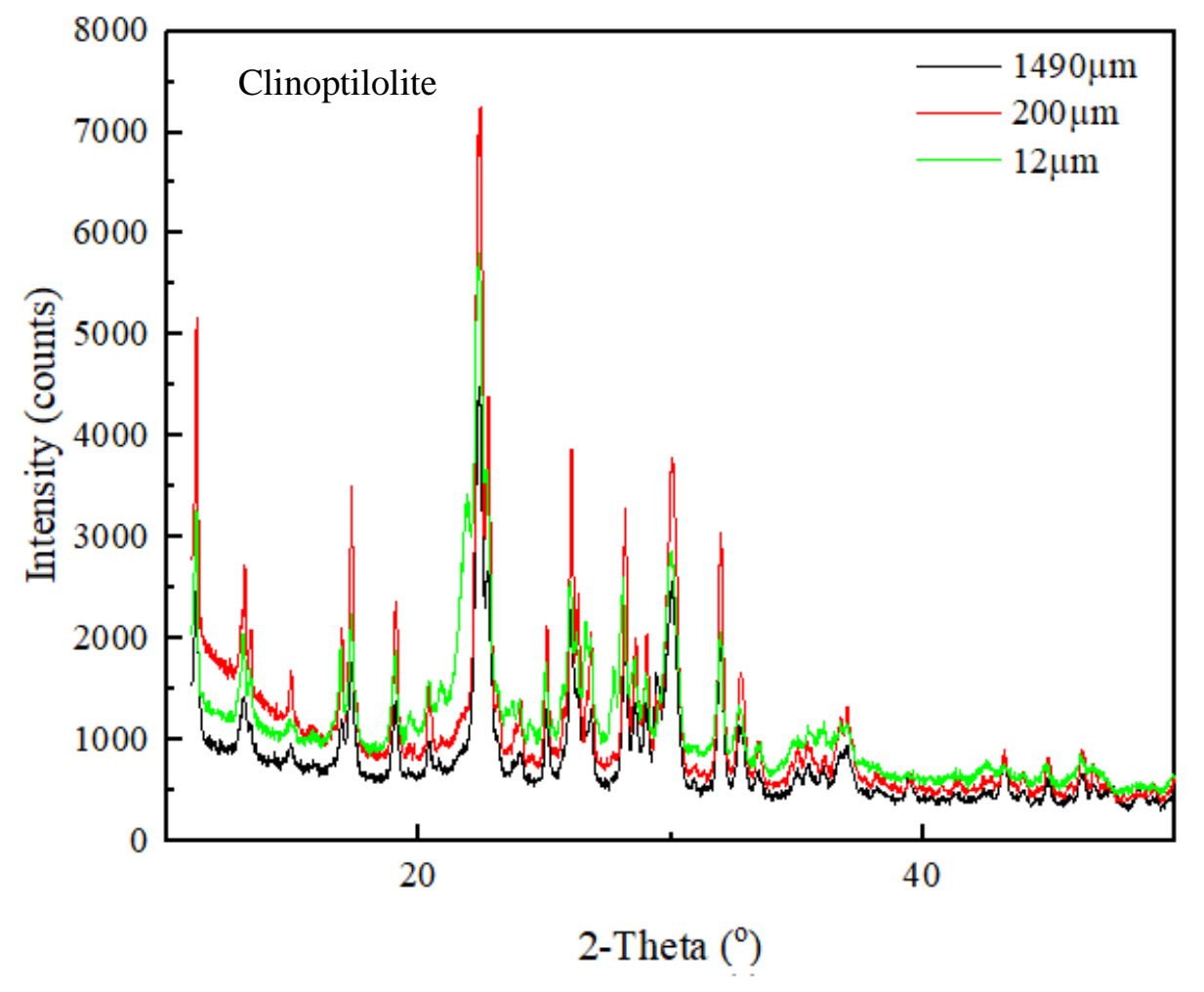

Figure $43 \mathrm{X}$-ray diffraction pattern of the zeolite samples.

XRD analysis was followed by identifying the chemical composition of the zeolite samples using the Vanta handheld XRF analyzer. Test results indicate that the samples contain a high concentration of silicon and aluminum, which is typical for zeolites. The distinctive properties of zeolites result from their honeycomb structures with water molecules and exchange cations located in the cavities. Mostly, $\mathrm{Ca}^{2+}, \mathrm{Na}^{+}$, or $\mathrm{K}^{+}$located in the cavities, are exchangeable with other ions depending on the $\mathrm{Si} / \mathrm{Al}$ ratio and the position of the ion exchange sites. This ratio provides a relatively high negative charge for attracting positive ions from the solution; thus, an increase in aluminum concentration will also provide higher adsorption capacity. The $\mathrm{SiO}_{2} / \mathrm{Al}_{2} \mathrm{O}_{3}$ ratio of the three zeolite samples was around $5.74 \%$. In addition to these major components, $1.43 \mathrm{wt} \% \mathrm{Ca}$, $0.99 \mathrm{wt} \% \mathrm{Fe}, 0.28 \mathrm{wt} \% \mathrm{Mg}$, and $2.10 \mathrm{wt} \% \mathrm{~K}$ were also detected in the samples as minor components. The framework structure containing cations and enclosing cavities, called supercage (Nandanwar et al., 2016). 
EDX spectrums of three zeolite samples are presented in a combined graph (Figure 44). All three samples represent a similar EDX pattern that shows $\mathrm{Si}, \mathrm{O}$, and $\mathrm{Al}$ as the main peaks. Compared to coarse and middle-sized zeolite samples, the EDX pattern of the finest zeolite sample $(12 \mu \mathrm{m})$ displays considerably high intensities for all element peaks. In addition to $\mathrm{Si}, \mathrm{Al}$, and $\mathrm{O}$, a portion of $\mathrm{Na}$ was also detected. The findings obtained from EDX, XRF, and XRD analyses complete and support each other. The carbon peak seen in the EDX spectrum is due to the carbon tape substrate used during sample preparation.

The images obtained by SEM under various magnifications are seen in Figure 45. As seen, regardless of the sample size, the surface is heterogeneous and rough with noticeable porous structures. Also, particles all have flaky and irregular shapes, which is consistent with the observations provided in Zendelska and his co-workers' study (2015). Besides, The finest zeolite samples also tend to agglomerate due to an increased surface area, as seen in Figure 45(C).

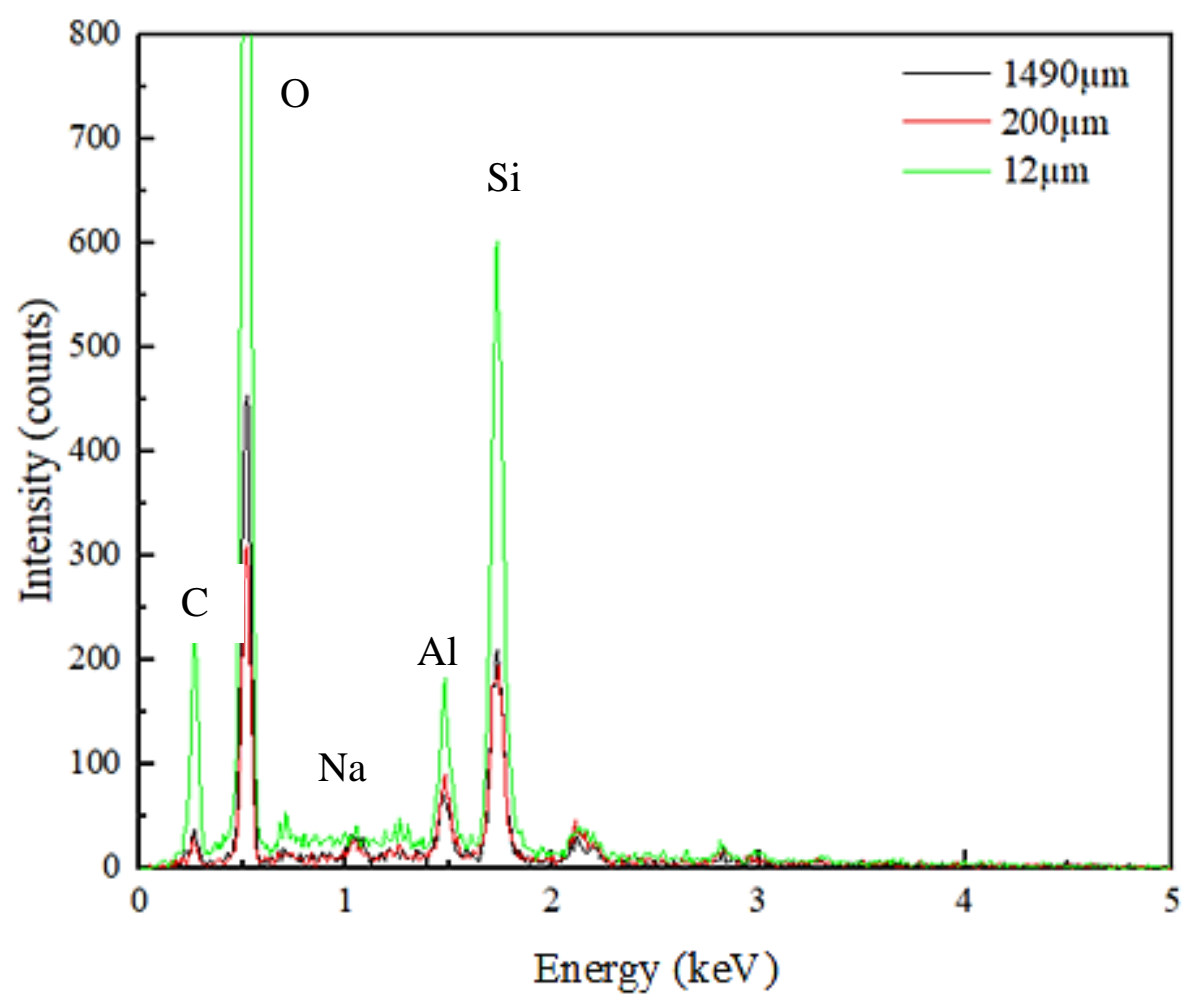

Figure 44 Elemental identification of the zeolite samples obtained from the EDX spectrums. 
A
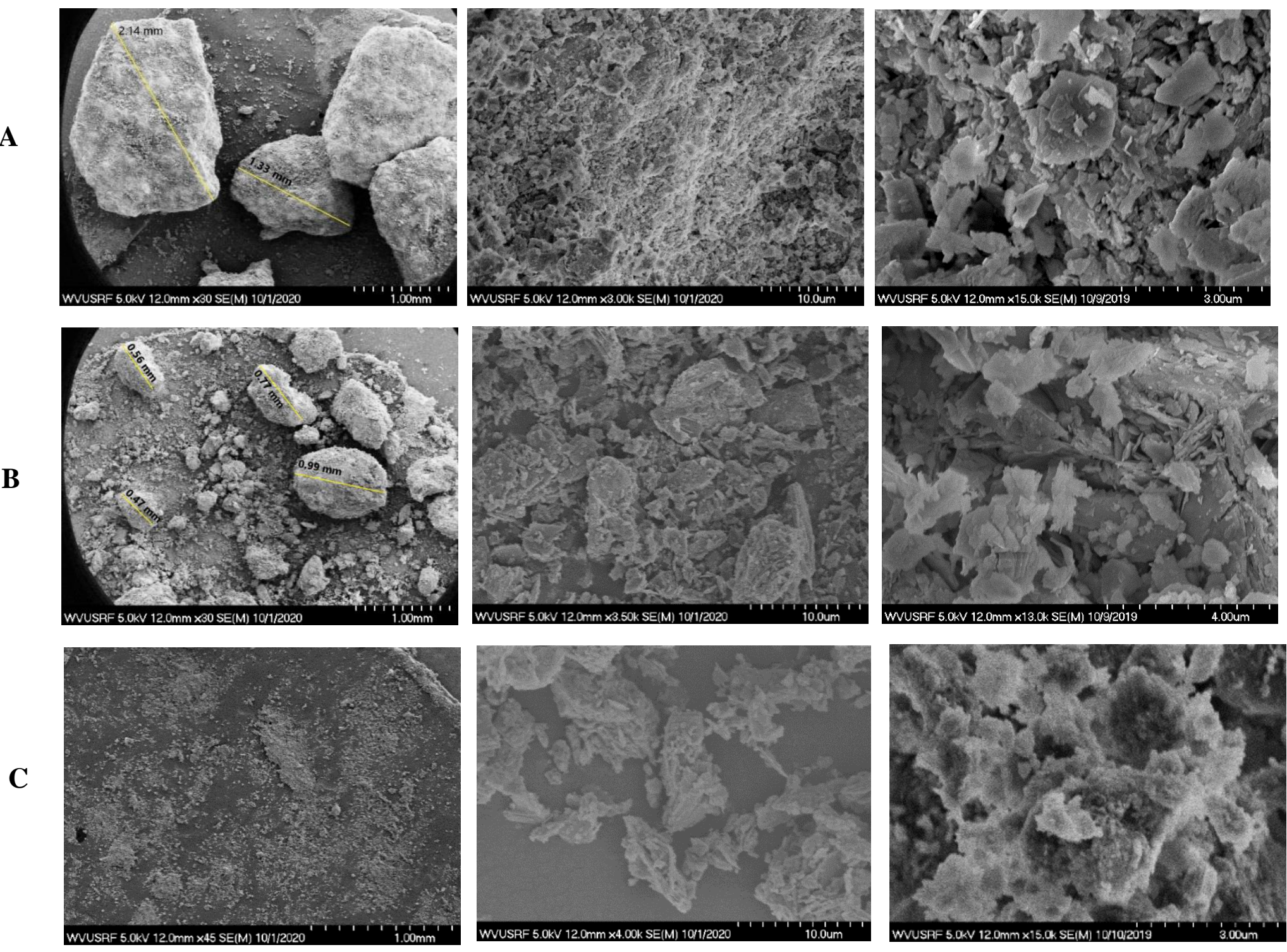

Figure 45 SEM images of the zeolite samples under various magnification. (a) the coarsest sample, (b) medium size sample, (c) the finest sample. 
Lastly, the three zeolite feed samples with a $\mathrm{P}_{80}$ value of $1490 \mu \mathrm{m}, 200 \mu \mathrm{m}$, and $12 \mu \mathrm{m}$, separately, were subjected to the BET nitrogen adsorption tests using the Micrometrics BET instrument equipped with the ASAP 2020 Version 4.02 to identify the pore size and specific surface area. The Brunauer-Emmett-Teller (BET) method is most widely used for evaluating the surface area of porous materials. Nitrogen (at $77 \mathrm{~K}$ ) is traditionally the adsorptive used to perform the analysis. While the coarsest zeolite had a pore diameter of $12.6 \mathrm{~nm}$, the middle-sized and the finest zeolites had a pore diameter of 9.9 and $8.7 \mathrm{~nm}$, respectively. According to the classification of pores established by the International Union of Pure and Applied Chemistry (IUPAC) in 1985 (Thommes et al., 2015), all zeolite samples have mesoporous structures due to pore widths less than $50 \mathrm{~nm}$. The specific surface area of the coarsest sample was determined as $16.1 \mathrm{~m}^{2} / \mathrm{g}$. The middle-sized and the finest samples had a specific surface area of 15.7 and $20.2 \mathrm{~m}^{2} / \mathrm{g}$, respectively. As seen, with a decrease in the particle size, the corresponding specific surface area generally increased. Moreover, the pore volume distribution as a function of the pore size indicated a linear distribution for all three zeolite samples.

The adsorption isotherm of the three zeolite samples is given in Figure 46, and the classification of physisorption isotherms is shown in Figure 47(A). According to the classification, the nitrogen adsorption on the zeolite sample is a Type IV (a) isotherm. Point $\mathrm{B}$, the beginning of the nearlinear section, usually corresponds to the completion of monolayer coverage. For example, a more gradual curvature, a less distinctive Point $\mathrm{B}$, is an indication of significant overlap of the monolayer coverage and the multilayer adsorption. There existed a linear relationship between the absorbance and the relative pressure varying from 0.09 to approximately 0.7 , representing the predominant mesoporous region of the fraction. A near-vertical adsorption line appeared with a relative pressure beyond 0.9 indicates that the zeolites had a portion of macropores as well. If a mesoporous adsorbent contains no macropores, the Type IV isotherm remains nearly horizontal over the upper range of $\mathrm{p} / \mathrm{p}^{0}$ (Thommes et al., 2015). In addition, the adsorption started at very low pressures, which may indicate the presence of some micropores (Ramesh et al., 2014). Besides, there existed a hysteresis between adsorption and desorption curves. This hysteresis occurs when the pore width exceeds a certain critical width (i.e., pores wider than $4 \mathrm{~nm}$ ) (Thommes et al., 2015). According to the hysteresis classification given in Figure 47(B), it is an H3 type of hysteresis. Pore-size distribution is usually expressed in the form of a pore volume distribution as the function of the pore size. As seen in Figure 48, the pore size distribution is linear. Overall, no difference was observed between the nitrogen adsorption behaviors of the three zeolite samples (i.e., coarse, middle-sized, and fine zeolite samples). 


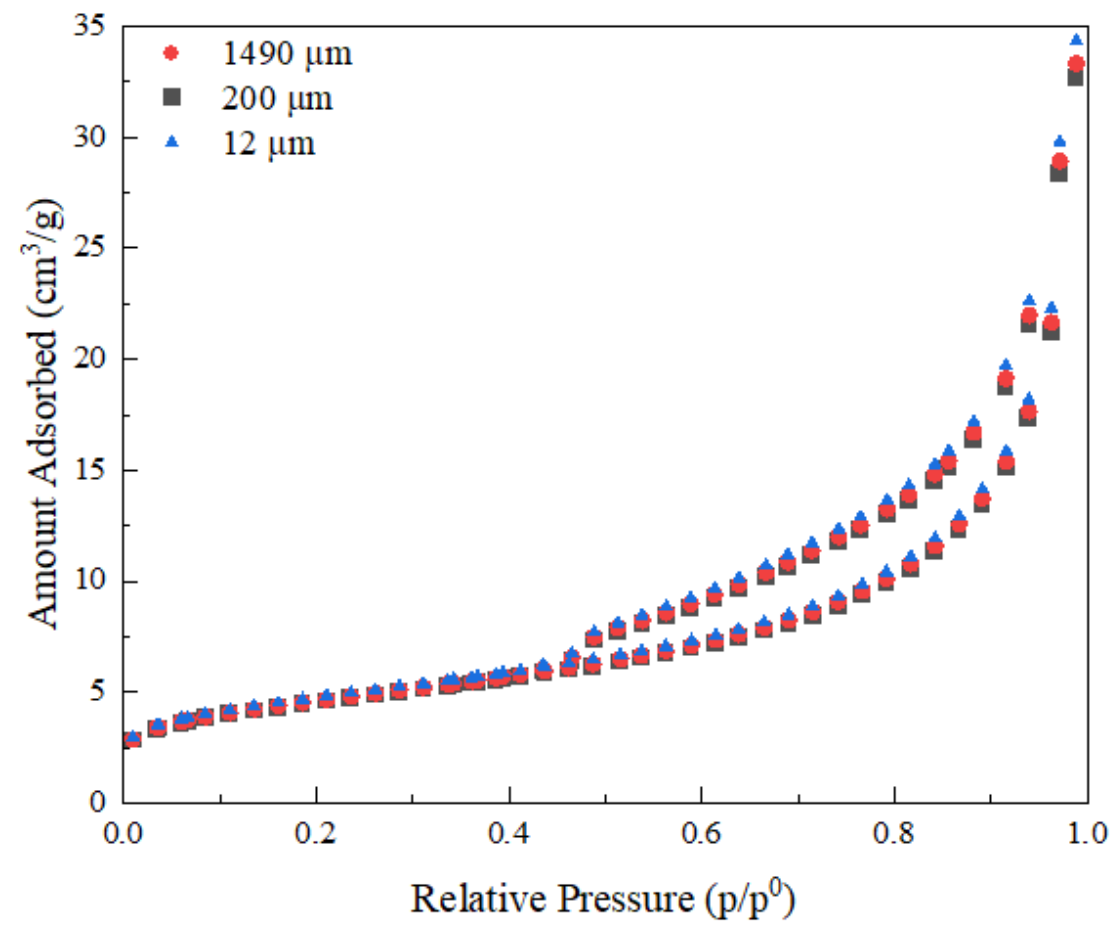

Figure 46 The Brunauer-Emmett-Teller (BET) Nitrogen adsorption isotherm of the three zeolite samples.

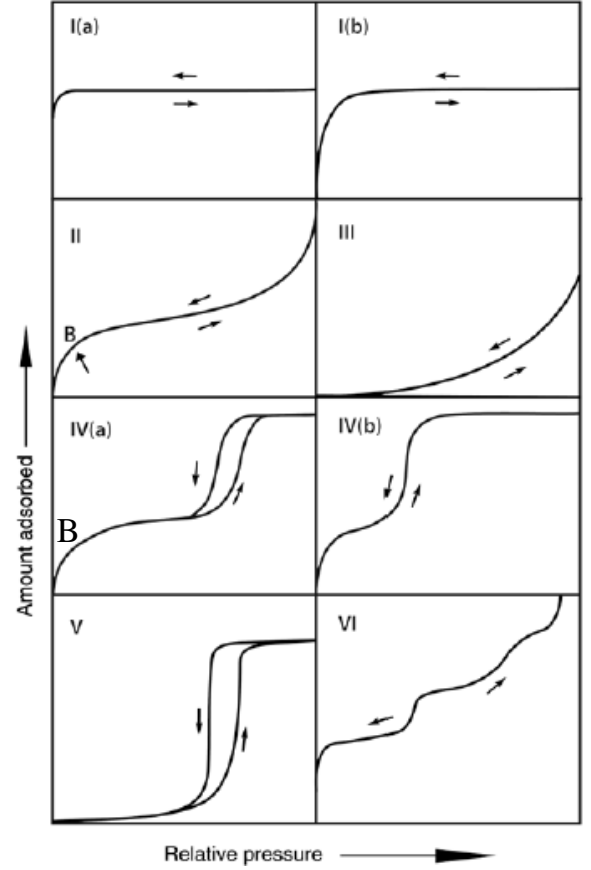

A

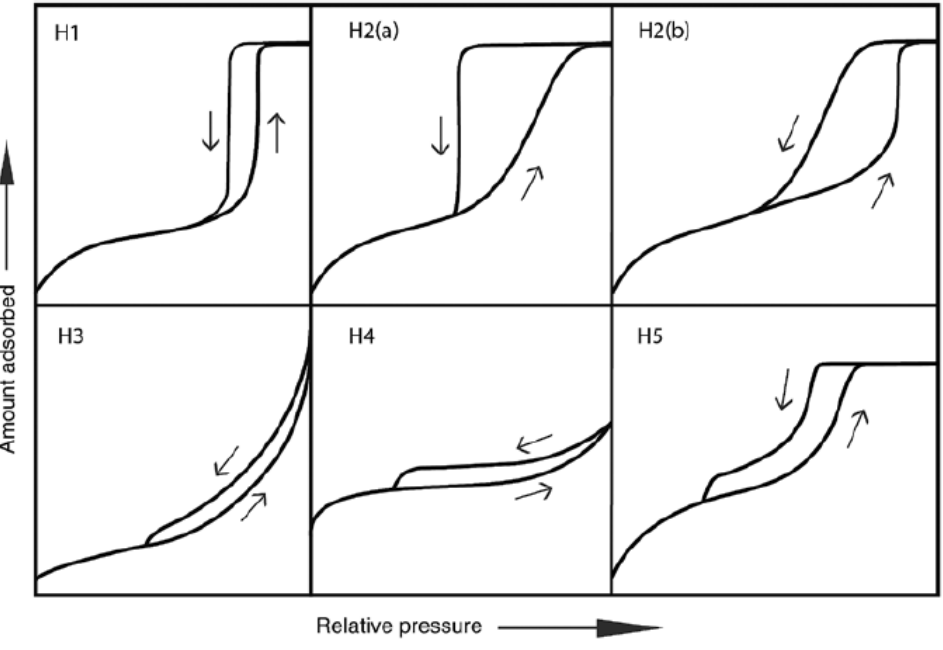

B

Figure 47 Classification of (a) physisorption isotherms, (b) hysteresis (Thommes et al., 2015) 


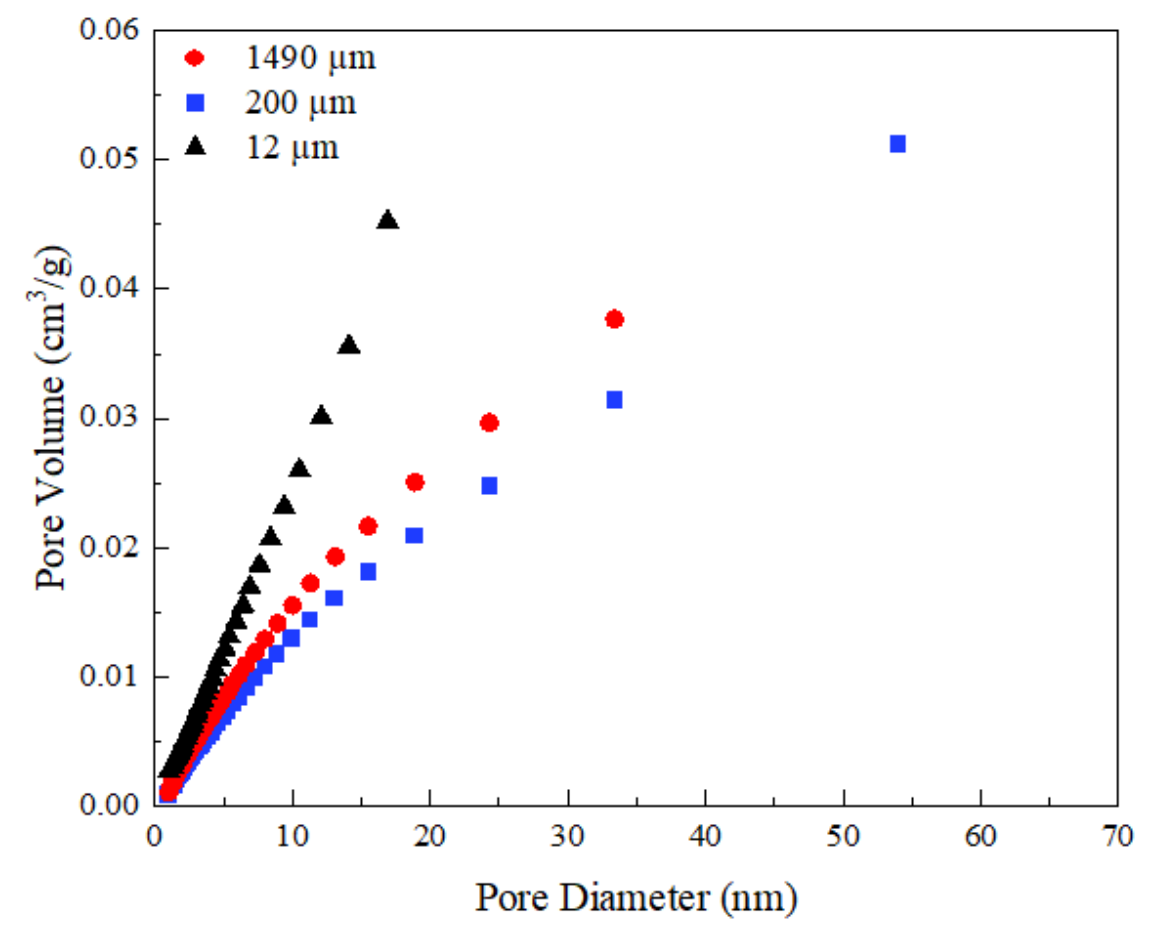

Figure 48 Pore size distribution of the three zeolite samples.

\subsection{Adsorption Tests}

The recovery of total rare earths, thorium, and uranium into the solid phase was given in Figure 49. As seen in Figure 49(A), the recovery of rare earths fluctuated between $0.03 \mathrm{wt} \%$ and 96.90 wt $\%$. On the contrary, nearly $100 \mathrm{wt} \%$ thorium removal was achieved in every test condition except for Test 23, which yielded a $36.67 \mathrm{wt} \%$ of thorium adsorption (Figure 49(B)). Like rare earths, a significant variation was observed for uranium adsorption, as shown in Figure 49(C). The adsorption recovery of uranium changed between 0.00 and $98.70 \mathrm{wt} \%$. 

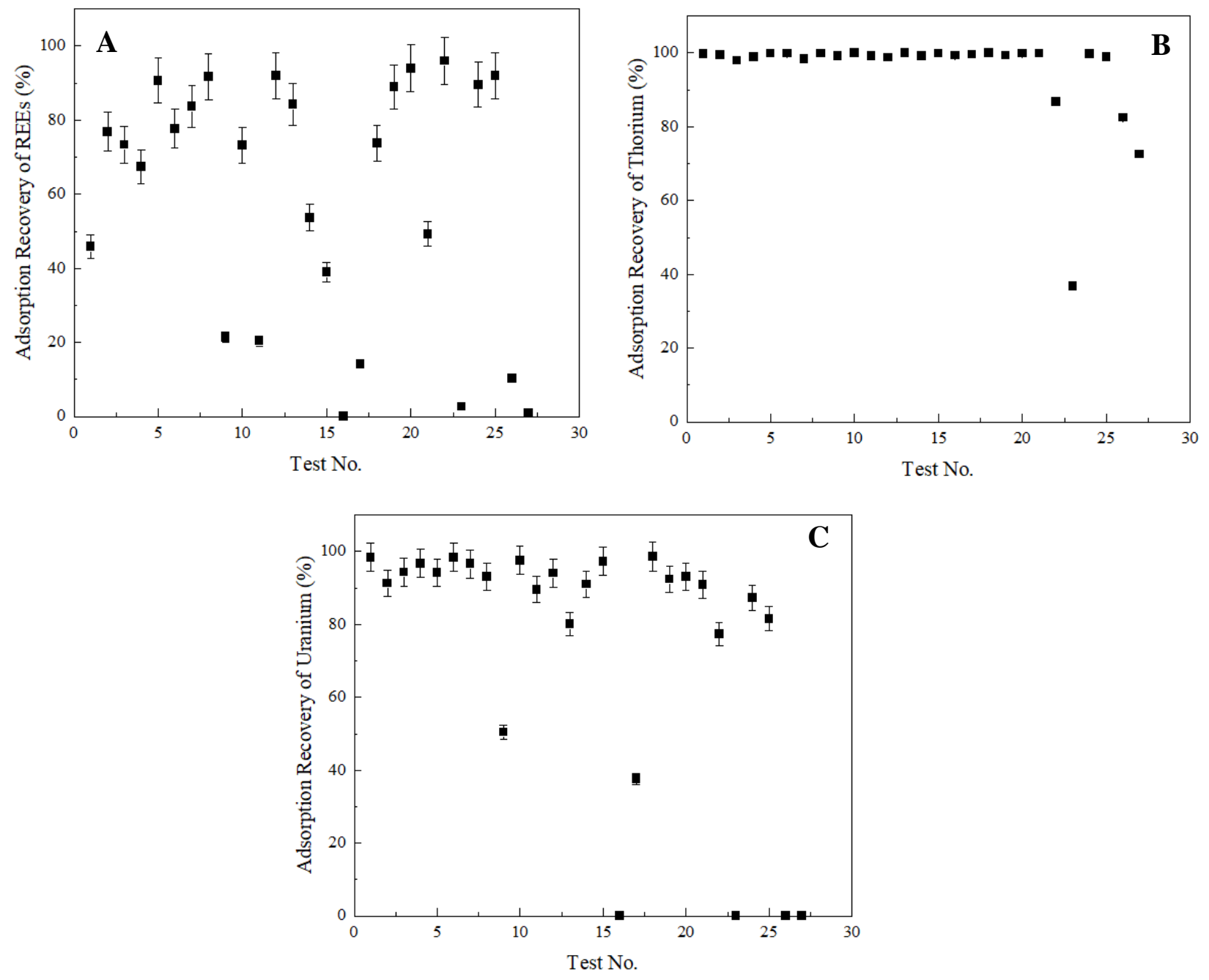

Figure 49 Adsorption recoveries into the solid phase. A) total rare earths, B) thorium, C) uranium. Error bars represent one standard deviation of multiple replicate test. 
The three lowest rare earth adsorption recoveries, namely $0.03 \mathrm{wt} \%, 1.01 \mathrm{wt} \%$, and $2.67 \mathrm{wt} \%$, were achieved with Test 16, 27, and 23, respectively, at the same $\mathrm{pH}$ value of 2 . Additionally, Test 16 and Test 27 were conducted with zeolite samples of the same size (i.e., $200 \mu \mathrm{m}$ ). However, Test 23 was performed using the zeolite sample with a $\mathrm{P}_{80}$ value of $12 \mu \mathrm{m}$, indicating the impact of particle size on the adsorption mechanism. The retention time of Test 16, 27, and 23 was 3, 1, and 2 hours, respectively. As seen, Test 16 produced the lowest rare earth adsorption into the solid phase $(0.03 \mathrm{wt} \%)$, which corresponded to a 3-hour contact time. However, when the zeolite's size was reduced to $12 \mu \mathrm{m}$ (Test 23), a retention time of 2 hours was sufficient to achieve a nearly equal low rare earth adsorption (2.67 wt\%). Moreover, in a study conducted by Kini (2006), it was stated that different adsorption tests might take various contact times to equilibrate with the ions in the solution to complete the adsorption. Although these three tests generated the lowest rare earth adsorption recoveries into the solid phase, no separation was observed between uranium and rare earths under the same conditions. This may be due to the saturation of adsorption sites with other ions before uranium. Under all three test conditions, $0 \mathrm{wt} \%$ uranium was adsorbed by the zeolites. On the other hand, thorium adsorption recovery varied among these three test conditions. The thorium adsorption recovery at Test 16, 27, and 23 was $99.33 \mathrm{wt} \%, 72.52 \mathrm{wt} \%$, and 36.67 wt $\%$, respectively. It is known that thorium shows minimal tendency to hydrolyze at low $\mathrm{pH}$ values, and the dominated ion at $\mathrm{pH}<3$ is $\mathrm{Th}^{4+}$, which is the exchangeable ion between solution and the zeolite. When the solution $\mathrm{pH}$ increases, the formation of other species (i.e. $[\mathrm{ThOH}]^{3+}$, $\left[\mathrm{Th}(\mathrm{OH})_{2}\right]^{2+}$, and $\left.\left[\mathrm{Th}(\mathrm{OH})_{3}\right]^{+}\right)$are seen due to hydrolysis. The hydroxyl group in these species increases with increasing $\mathrm{pH}$ (Faghihian et al., 2004).

The effect of particle size was expected since adsorption capacity is dependent on the effective surface area, which generally increases with decreasing particle size (Zou et al., 2009). Also, particles with a smaller diameter are characterized by a larger specific surface area. Thus the adsorption is enhanced, and a shorter contact time is required to reach the equilibrium. However, an increase in the adsorption recovery of rare earths was observed while a coarser zeolite sample was used. For example, Test 5 and Test 11 were conducted under the same operating conditions, except for the zeolite particle size. While Test 5 was performed with the coarsest zeolite, $1490 \mu \mathrm{m}$, Test 11 was carried out with the finest size zeolite, $12 \mu \mathrm{m}$. The respective rare earth adsorption recoveries were found as $90.67 \mathrm{wt} \%$ and $20.43 \mathrm{wt} \%$. The reason for this substantial increase in the adsorption recovery of rare earths may lie under the particle size reduction occurring during the mixing of solid and liquid phases throughout the adsorption process. The post adsorption SEM image provided in Figure 50 shows fractures and cracked surfaces, which may result from a reduction in the zeolite particle size. Later, the particle size analysis performed with post adsorption solid samples confirmed the size reduction. The particle size of the coarsest zeolite sample with an initial $\mathrm{P}_{80}$ value of $1490 \mu \mathrm{m}$ was reduced to $45 \mu \mathrm{m}$ after adsorption. Likewise, a reduction in the particle size of the middle-sized zeolite sample was also observed. After adsorption, the new $\mathrm{P}_{80}$ value of the middle-sized zeolite sample was found to be $60.5 \mu \mathrm{m}$ as opposed to the initial value of $200 \mu \mathrm{m}$. However, no change in the particle size of the fine sample (i.e., $12 \mu \mathrm{m}$ ) was detected. The particle size reduction occurred for coarse and middle-sized zeolite 
samples during adsorption. It created fresh surfaces and new active sites ready for ion adsorption that may explain the elevated adsorption recovery with increased nominal adsorbent particle size.

Overall, the best separation performance was achieved with Test 11 while using 2.50 grams of 12$\mu \mathrm{m}$ zeolite sample at a $\mathrm{pH}$ value of 3 with a contact period of 2 hours. Under these conditions, the adsorption recovery of rare earths, thorium, and uranium into the solid phase was found to be 20.43 $\mathrm{wt} \%, 99.20 \mathrm{wt} \%$, and $89.60 \mathrm{wt} \%$, respectively.

In terms of major metal adsorption, steady recovery results were observed for iron in comparison to aluminum and magnesium. Almost $100 \mathrm{wt} \%$ iron adsorption onto zeolite was seen under every test condition. Similar selective iron adsorption over other metals was seen in Motsi and his coworkers' (2009) study on heavy metal adsorption from acid mine drainage by natural zeolite. Contrarily, the adsorption recovery of aluminum changed between $12.49 \mathrm{wt} \%$ to $99.65 \mathrm{wt} \%$, where the lowest adsorption recovery was achieved with Test 11 . The adsorption recovery of magnesium also varied. The lowest magnesium adsorption, $69.60 \mathrm{wt} \%$, was observed with Test 11, and the highest magnesium adsorption recovery seen was $99.87 \mathrm{wt} \%$. Detailed results for the adsorption studies are provided in Table 36 and Table 37 in the Appendix.

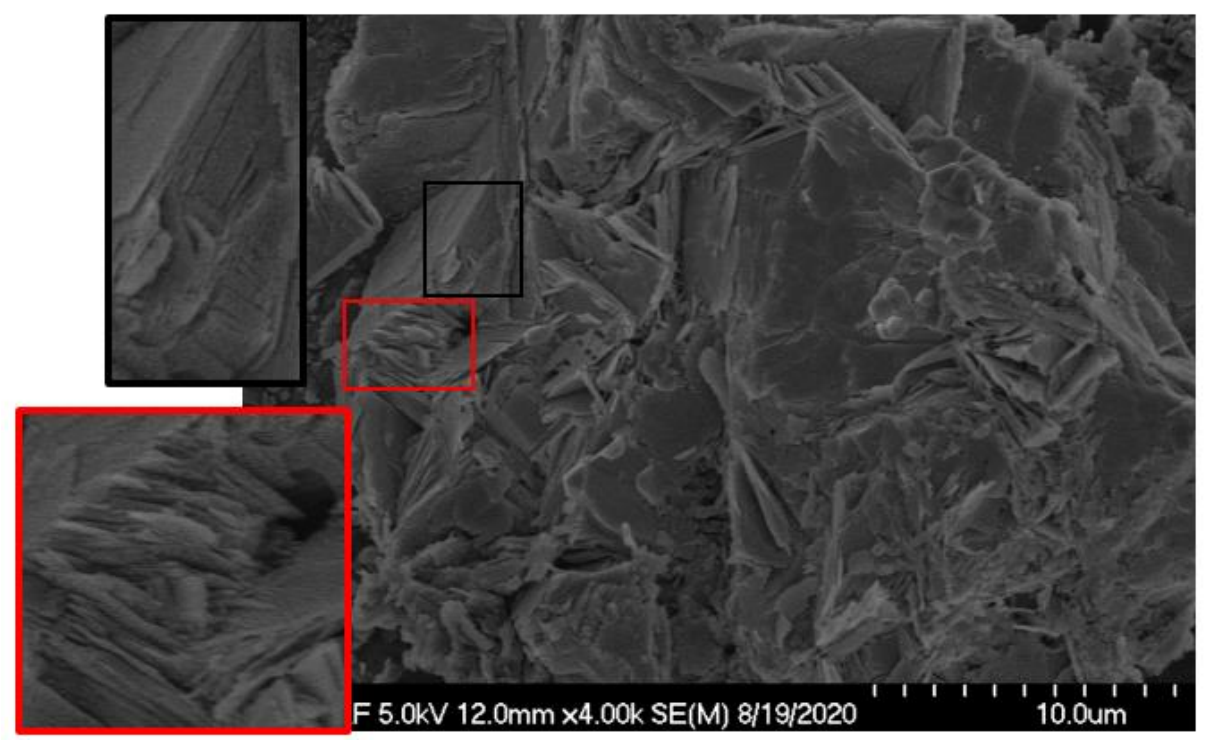

Figure 50 SEM image showing the fractures and cracks formed after adsorption.

\subsection{Characterization of Post Adsorption Zeolite Samples}

The solid sample used during Test $11(12-\mu \mathrm{m}$ zeolite) was subjected to XRD and SEM-EDX analyses to detect any change in the elemental and mineralogical compositions after adsorption. Results obtained from XRD revealed the formation of halite and perrierite-Ce in addition to clinoptilolite. Halite formation is probably due to the reaction between feedstock's medium, $\mathrm{HCl}$, and the addition of $\mathrm{pH}$ regulator, $\mathrm{NaOH}$. Also, the zeolite samples initially contained a small amount of sodium before the adsorption tests. Perrierite-Ce is a compound consisting of many elements and having a general formula of $\mathrm{A}_{4} \mathrm{BC}_{2} \mathrm{D}_{2} \mathrm{Si}_{4} \mathrm{O}_{22}$, where $\mathrm{A}$ represents rare earths and 
thorium, $\mathrm{B}$ represents $\mathrm{Fe}, \mathrm{Mn}, \mathrm{Mg}, \mathrm{C}$ refers to $\mathrm{Fe}, \mathrm{Mn}, \mathrm{Mg}, \mathrm{Al}, \mathrm{Ti}, \mathrm{Cr}$, and $\mathrm{D}$ corresponds to $\mathrm{Ti}$ (Muhling et al., 2014). Cerium and lanthanum are the most dominant rare earth elements. Perrierite-Ce is defined as radioactive according to the Code of Federal Regulations Title 49, which supports the high concentration of thorium in the zeolite post adsorption. The XRD pattern of the sample was given in Figure 51.

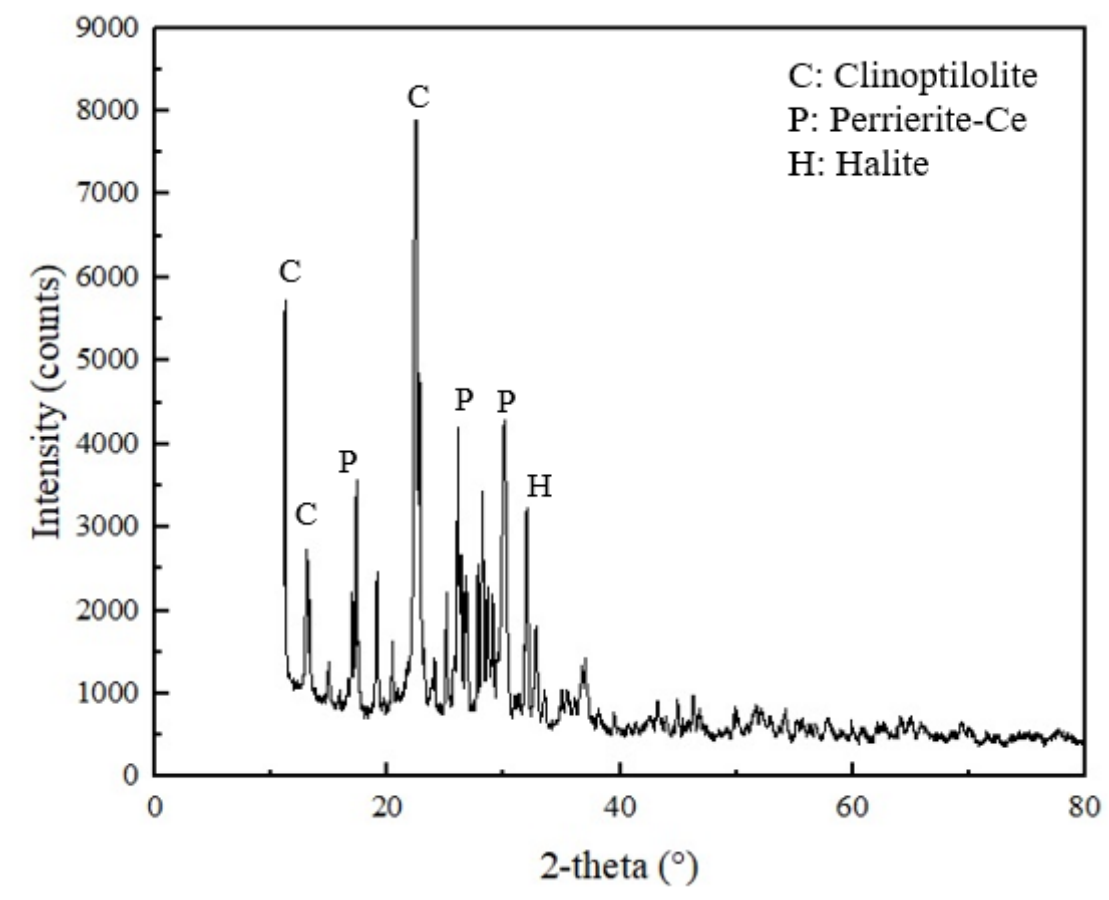

Figure 51 XRD pattern of the solid samples generated with Test 11.

Image analysis was performed using SEM to provide further information. As seen in Figure 52, the morphology remains rough, and the particles maintain irregular shapes. However, the formation of small prismatic crystal particles is seen. This finding is well correlated with the crystal structure of perrierite-Ce (Chukanov et al., 2012). The bright areas highlighted in the image may refer to the accumulation of heavy elements such as the rare earths and thorium.

The EDX pattern of the solid sample generated from Test 11 is shown in Figure 53(A). In comparison to the elemental concentration of the raw zeolite samples, after adsorption, a substantial decrease in the intensities of aluminum and silicon was observed. This finding suggests a reduction in aluminum and silicon concentration, attributed to the adsorption of external elements onto the zeolite structure. Like XRD, EDX was also able to detect thorium and most of the rare earth elements; however, their concentrations are considerably low compared to $\mathrm{Al}, \mathrm{Si}$, and $\mathrm{O}$. Due to this fact, they are not clearly seen in the full pattern. However, as shown in the close-up view (Figure 53(B)), most of the rare earths were observed in the energy level between 0.5 and $1.3 \mathrm{keV}$, and thorium was detected around $3 \mathrm{keV}$ 


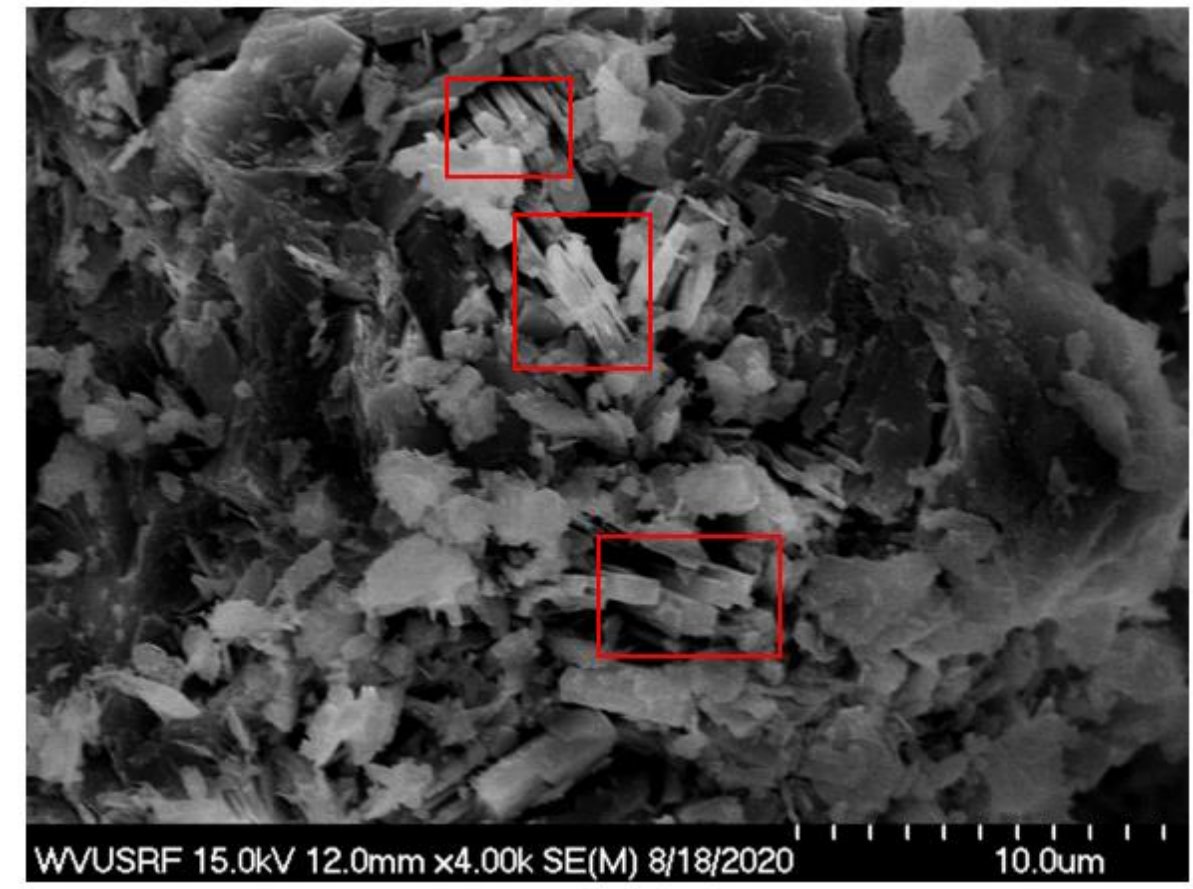

Figure 52 SEM images of the solid samples generated after adsorption. Bright areas highlighted in the red boxes indicating the deposition of heavy elements.
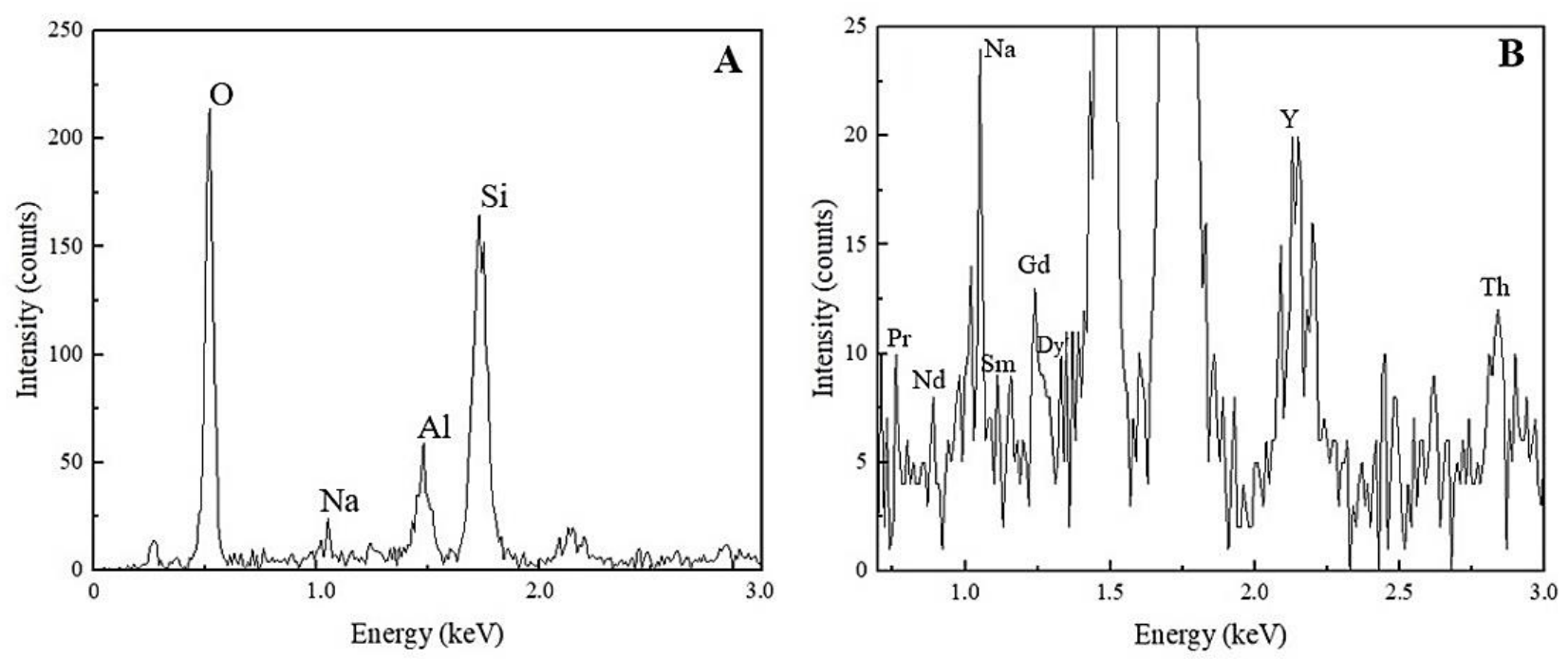

Figure 53 EDX pattern of the solid sample generated after Test 11. (A) Full EDX pattern indicating high-intensity elements. (B) Close-up view showing the detection of rare earths and thorium. 


\subsection{Statistical Analysis and Modeling of Adsorption Tests}

Following the test results, the impact of four operating variables (i.e., zeolite particle size, feed solution $\mathrm{pH}$, adsorbent amount, and contact time) on the recovery of total rare earths, thorium, and uranium was analyzed using the response surface methodology. The adsorption recovery values of rare earths, thorium, and uranium obtained from the full dataset were uploaded to the Design Expert Software following the Box Behnken experimental design. Statistical models were developed separately to determine the operational sensitivity with respect to the input parameters.

A statistical model was first developed as a tool to predict the recovery of rare earth elements by incorporating all the influential operating parameters, as shown in Eq. (33). It can be seen from the quadratic model that the variables, including the zeolite particle size, feed solution $\mathrm{pH}$, and adsorbent amount, except the contact time, all had a significant impact on the recovery of rare earths.

REE Adsorption Recovery $=+60.21+28.43 * A+27.46 * B+16.27 * C+8.58 * D-14.33 *$ $A B-5.08 * A C-3.48 * A D-14.13 * B C+5.62 * B D-14.23 * C D+2.08 * A^{2}-7.39 * B^{2}+$ $7.76 * C^{2}-3.68 * D^{2}$

where the uncoded model terms A, B, C, and D correspond to zeolite particle size, feed solution $\mathrm{pH}$, adsorbent amount, and contact time, respectively. While a statistical model was generated for rare earths, a significant model was generated for neither thorium nor uranium.

The Analysis of Variance (ANOVA) table used to determine the significance of the statistical model generated for the estimation of rare earth adsorption recovery is shown in Table 21. As seen from the table, the model F-value of 6.90 implies the model is significant. The probability of an F-value becoming this large is $0.09 \%$ ( $<5 \%$, which is the critical P-value used to determine the significance). Model terms are considered significant within a $95 \%$ confidence level, or in other words, values of "prob $>F$ " less than 0.05 indicate that terms are significant. In this case, terms A, $\mathrm{B}$, and $\mathrm{C}$ are significant model terms, whereas $\mathrm{D}$ is an insignificant model term. The lack of fit value of 1.21 implies a $53.50 \%$ chance that a lack-of-fit F-value can be this high. The lack of fit must be insignificant for a model to be fit. In the same table, R-squared values were also given to further evaluate the robustness of the developed model. As seen, the R-squared value of 0.8895 , and an adjusted R-squared value of 0.7606 further confirm the model's significance. 
Table 21 ANOVA analysis of the quadratic model for predicting rare earth recovery.

\begin{tabular}{ccccccc}
\hline Source & $\begin{array}{c}\text { Sum of } \\
\text { Squares }\end{array}$ & df & $\begin{array}{c}\text { Mean } \\
\text { Square }\end{array}$ & F Value & $\begin{array}{c}\text { p-value } \\
\text { Prob> F }\end{array}$ & \\
\hline Model & 26572.63 & 14 & 1898.05 & 6.90 & 0.0009 & Significant \\
A- Particle Size & 9696.90 & 1 & 9696.90 & 35.25 & 0.0001 & \\
B- pH & 9048.07 & 1 & 9048.07 & 32.89 & 0.0001 & \\
C- Adsorbent & 3177.21 & 1 & 3177.21 & 11.55 & 0.0053 & \\
AB & 821.68 & 1 & 821.68 & 2.99 & 0.1095 & \\
AC & 103.12 & 1 & 103.12 & 0.37 & 0.5518 & \\
BC & 798.06 & 1 & 798.06 & 2.90 & 0.1142 & \\
A2 & 23.08 & 1 & 23.08 & 0.084 & 0.7770 & \\
B2 & 290.94 & 1 & 290.94 & 1.06 & 0.3240 & \\
C2 & 320.78 & 1 & 320.78 & 1.17 & 0.3014 & \\
Residual & 3300.85 & 12 & 275.07 & & & \\
Lack of Fit & 2832.19 & 10 & 283.22 & 1.21 & 0.5350 & Not Significant \\
Pure Error & 468.66 & 2 & 234.33 & & & \\
Cor Total & 29873.48 & 26 & & & & \\
R-Squared & 0.8895 & & & & & \\
Adj R-Squared & 0.7606 & & & & & \\
Pred R-Squared & 0.4186 & & & & & \\
Adeq Precision & 10.110 & & & & & \\
\hline
\end{tabular}

Three diagnostic plots developed based on the statistical model are given in Figure 54. The relationship between two parameters was described in these plots while keeping the other two parameters constant at their middle values. In Figure 54(A), the effect of particle size and $\mathrm{pH}$ is presented while keeping the adsorbent amount and contact time constant at $3.75 \mathrm{~g}$ and $2 \mathrm{hrs}$, respectively. It is well-known that $\mathrm{pH}$ is an important parameter in adsorption processes. As seen, rare earth recovery increases with an increase in the $\mathrm{pH}$ value. This phenomenon could attribute to the competition between hydrogen ions and rare earth ionic species present in the solution at a given $\mathrm{pH}$ value (Faghihan et al., 2004). Typically, zeolite has a tendency to adsorb $\mathrm{H}^{+}$ions from the solution, which creates a positive charge on the zeolite structure and other positively charged ions are repulsed due to the electrostatic interaction. When the solution $\mathrm{pH}$ increases, the amount of $\mathrm{H}^{+}$ions decreases, which results in the adsorption of other ions present in the solution (Motsi et al., 2009). However, freed negatively charged ions (i.e., $\mathrm{Cl}^{-}$) may reverse the electrostatic repulsion and enhance adsorption. At the same time, depending on the concentration and conditions, it may have an opposite effect and further reduce the adsorption by binding with metals and forming complexes that cannot be adsorbed. This fact could be the reason for the wide variation observed in the adsorption recovery of rare earths. Also, rare earth adsorption recovery rises with an increase in the particle size, which statistically supports the findings stated earlier that unexpected particle size reduction induced during mixing leads to enhanced adsorption due to freshly created surfaces for ion adsorption. The same trend was seen in Figure 54(B). The relationship between particle 
size and the adsorbent amount is given while maintaining the $\mathrm{pH}$ and contact time constant at 2 and 2 hours, respectively. In comparison to Figures 54(A) and 54(C), Figure 54(B) displays a relatively flat surface. A higher amount of adsorbent will result in more significant adsorption because the active adsorption sites will increase concurrently. Figure 54(C) further shows that rare earth recovery decreases at a lower $\mathrm{pH}$ value and a smaller adsorbent amount. 


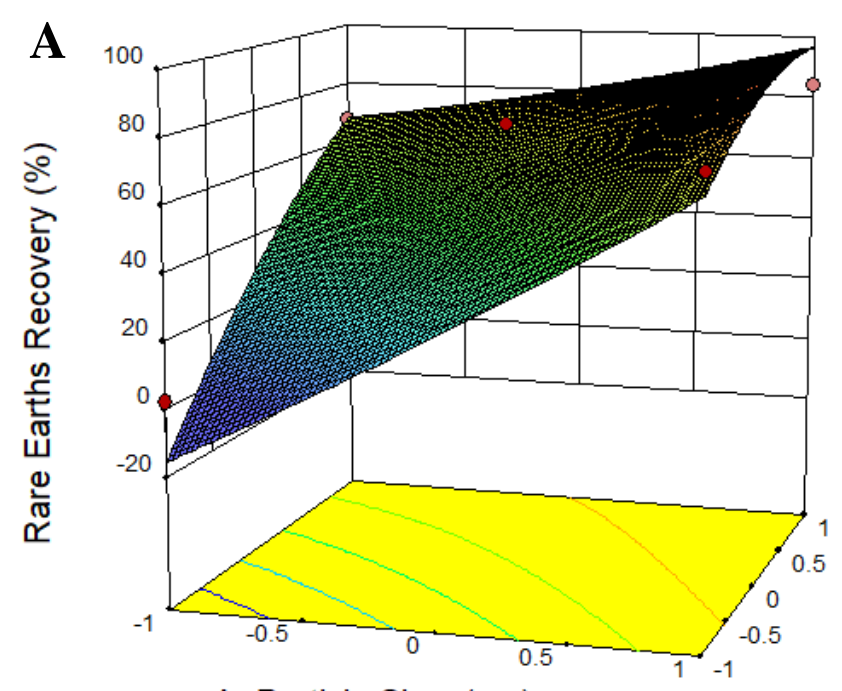

A: Particle Size $(\mu \mathrm{m})$

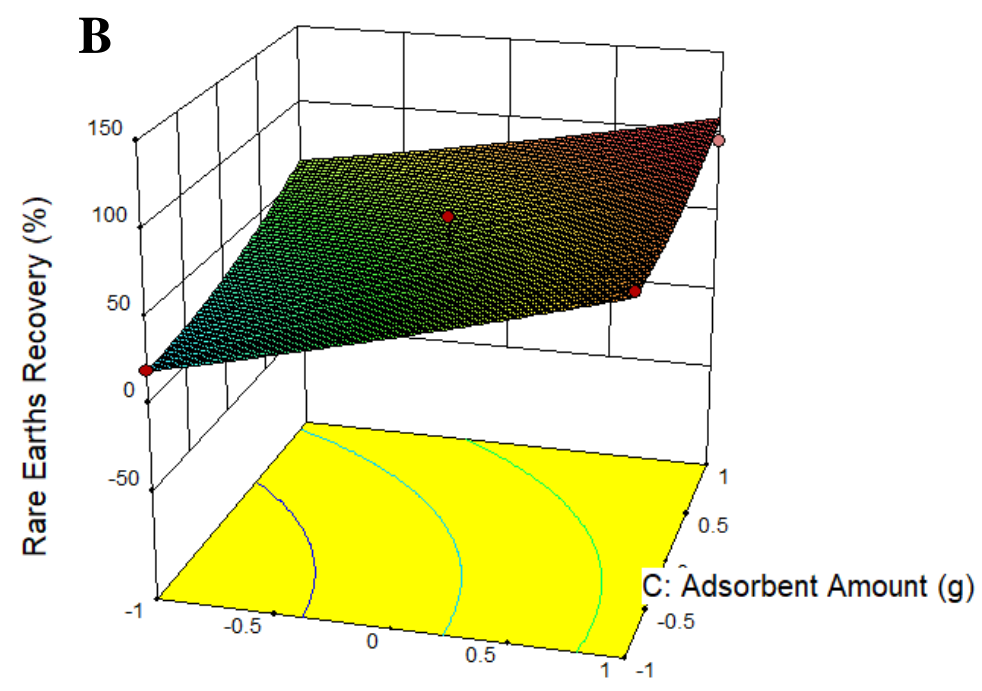

A: Particle Size $(\mu \mathrm{m})$

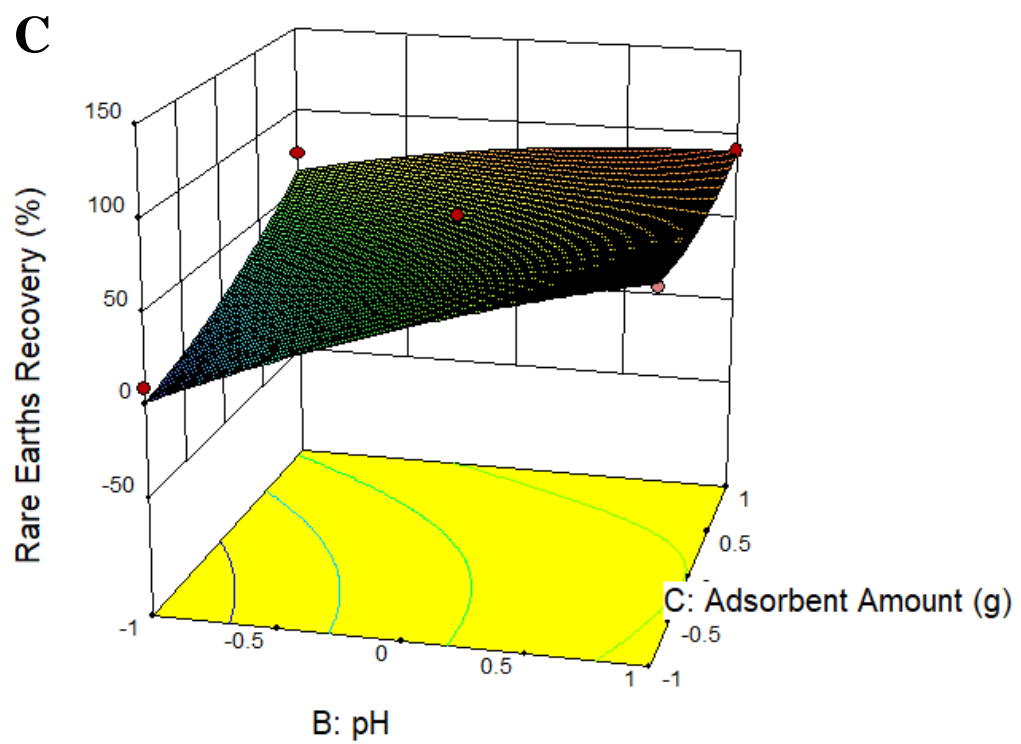

Figure 54 Diagnostic plots obtained based on the statistical model developed for the adsorption recovery of rare earths. 


\subsection{Adsorption Kinetics and Isotherms}

The kinetic adsorption test results shown in Figure 55 indicate fast adsorption for thorium instead of rare earths and uranium. Within the first $5 \mathrm{~min}$, the adsorption recovery of thorium reached 86.5 wt $\%$, which later continued to rise but with a slight incremental increase. At the end of the $3-\mathrm{hr}$ contact, the maximum thorium adsorption onto the zeolite was $93.4 \mathrm{wt} \%$. On the other hand, rare earths and uranium followed a similar pattern jointly. A significant increase in the adsorption recovery of rare earths was first seen during the 10-15 min time interval. At the end of $10 \mathrm{~min}$, the adsorption recovery of rare earths was $3.0 \mathrm{wt} \%$, and at $15 \mathrm{~min}$, it increased by $8.2 \mathrm{wt} \%$ and reached a new value of $11.3 \mathrm{wt} \%$. Later, another $8.2 \mathrm{wt} \%$ increase was further observed during the 30-45 min time interval. Adsorption recovery of total rare earths reached $21.5 \mathrm{wt} \%$ at the end of the kinetic test. Data shown in Figure 56 further support the findings mentioned above. It can be seen from Figure 56(A) that the distribution of rare earths in the solid phase increased significantly at both the 10-15 and 30-45 min time intervals. The distribution of thorium as a function of adsorption time is presented in Figure 56(B), which again suggests that the most significant adsorption occurred during the first $5 \mathrm{~min}$. Uranium exhibited a similar adsorption behavior as rare earths. Low uranium adsorption was also observed in Godelitsas and Armbsruster's study due to the complicated aqueous chemistry of uranium as well as the constant structural changes occurring in the mesoporous zeolite sample (Godelissas and Armbruster, 2003). In the same study, high thorium adsorption along with hydrogen ion adsorption at $\mathrm{pH}<3$ were also observed, which shares similar findings presented here. The adsorption mechanism starts with the formation of complexes at the external surface. Later, the ions transport into the pores to form inner complexes via covalent or ionic bonding and become more stable. As the valence of a cation increases, the tendency of complexation also increases. Later the cation creates an outer-sphere complex in which at least one water molecule being in between the cation and the adsorbent. Sometimes, the cation, which is surrounded by water molecules, may locally neutralize the surface charge of the adsorbent instead of forming a complex. In that case, the ion lies in the diffusion layer. While the complexes on the Stern layer may have covalent or ionic bonding, the ions in the diffusion layer only have ionic bonds. Rapid thorium complexes formed on the external surface of the zeolite may block the formation of complexes with other ions. Afterward, the thorium molecules diffusing into the inner pores can hinder the adsorption of other ions. This may also be explained via the molecule sizes of rare earths, thorium, and uranium. While thorium has a molecule size of $0.24 \mathrm{~nm}$, uranium has a molecule size of $0.23 \mathrm{~nm}$. Although the molecule size of rare earths changes, it varies between 0.23 and $0.25 \mathrm{~nm}$. If thorium is adsorbed first due to its easy nature, it may slow down the transport of other ions when the adsorbent has a narrow pore diameter (Malamis and Katsou, 2013). This matter also supports the identification of thorium-containing mineral phases in the XRD analysis conducted after adsorption. Moreover, Parzentny and Rog (2019) stated that uranium is mainly concentrated on the surface of Al-Si porous structures, and thorium is concentrated in the Al-Si grains. 


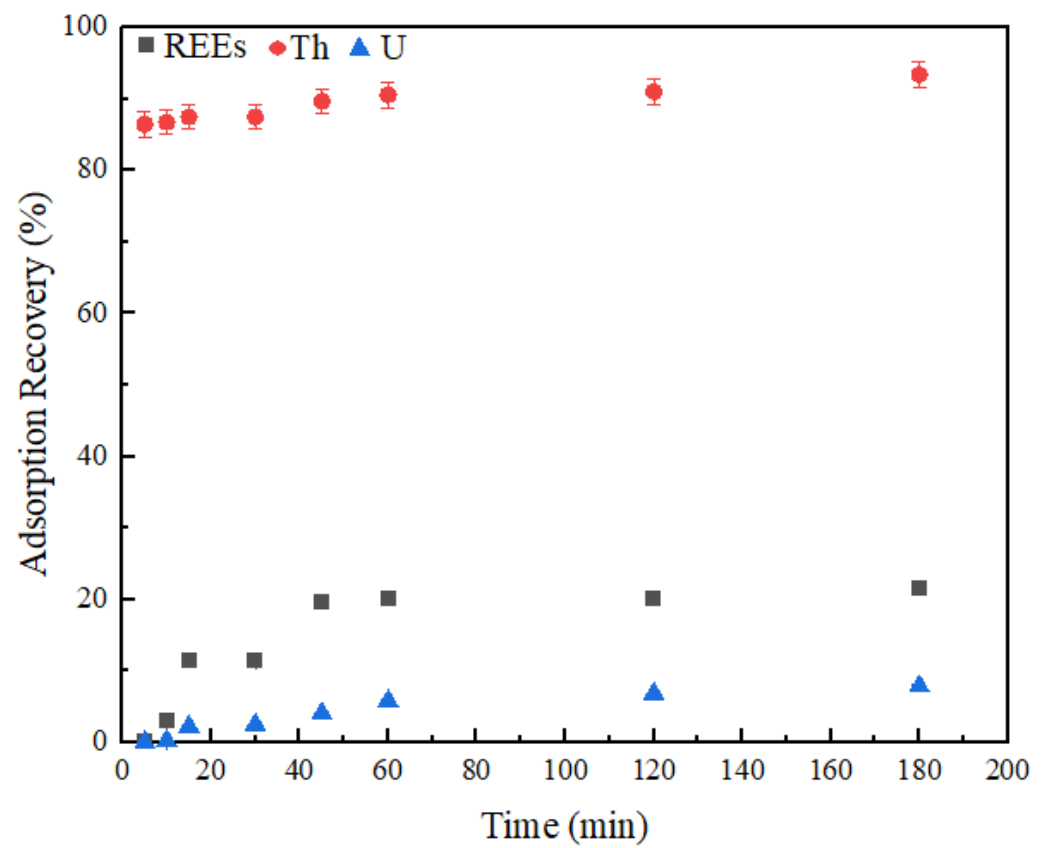

Figure 55 Adsorption recoveries of total rare earths, thorium, and uranium as a function of contact time. Error bars represent one standard deviation of multiple replicate tests.
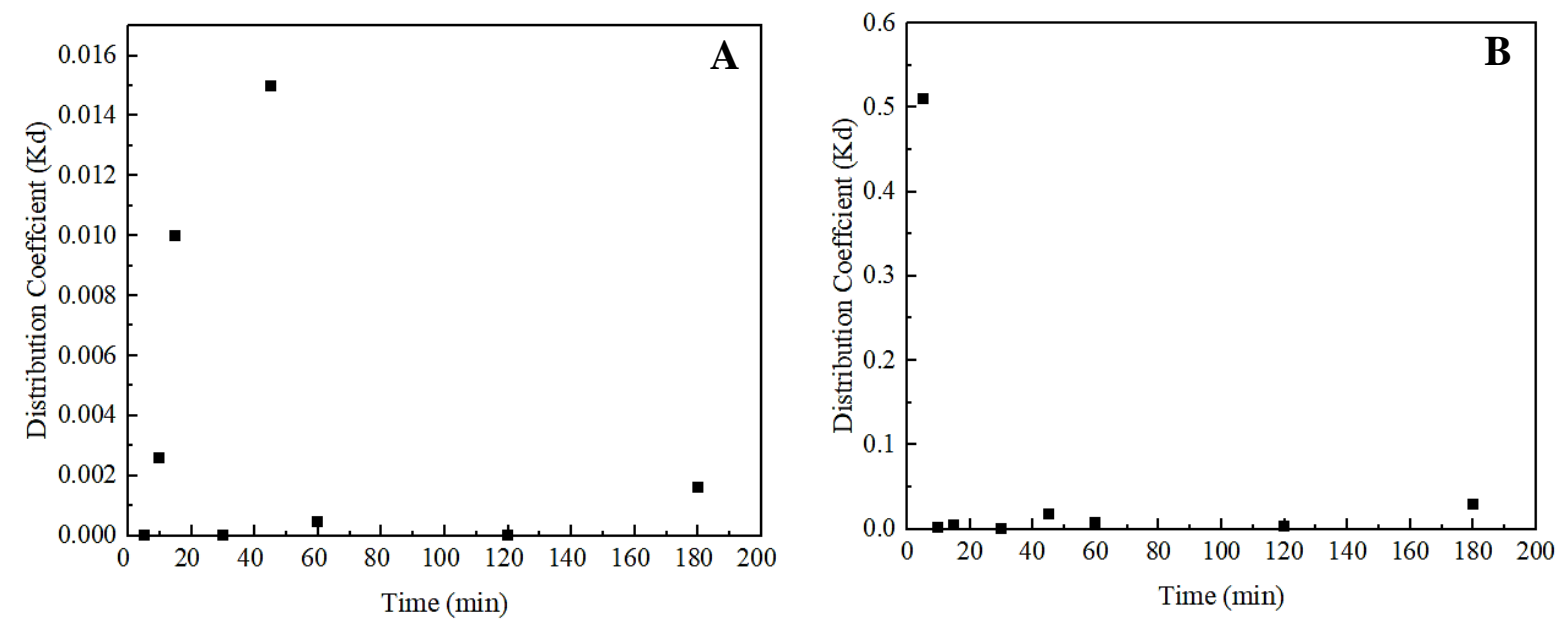

Figure 56 Distribution coefficient values of (A) total rare earths and (B) thorium as a function of contact time. Error bars represent one standard deviation of multiple replicate tests.

The adsorption data were examined according to the kinetic models of pseudo-first-order (equation 34) and pseudo-second-order (equation 35). The pseudo-first-order model assumes that the rate of radionuclide removal with time is directly proportional to the difference between the equilibrium capacity of sorbent and the amount of radionuclide sorbed at any time. On the other hand, the pseudo-second-order model assumes that the sorption process is a pseudo-chemical reaction process which means the driving force is not the difference between the equilibrium capacity of 
sorbent and the amount of radionuclide sorbed at any time, but the overall sorption rate is proportional to the square of the driving force (Sani et al., 2015).

$$
\begin{aligned}
& \ln \left(q_{e}-q_{t}\right)=\ln q_{e}-k_{1} t \\
& \frac{t}{q_{t}}=\frac{1}{k_{2} q_{e}^{2}}+\frac{1}{q_{e}}
\end{aligned}
$$

Where $\mathrm{q}_{\mathrm{t}}$ and $\mathrm{q}_{\mathrm{e}}(\mathrm{mg} / \mathrm{g})$ are the amounts of ions adsorbed at $\mathrm{t}(\mathrm{min})$ and equilibrium, separately. $\mathrm{k}_{1}$ and $\mathrm{k}_{2}$ are the rate constants $(1 / \mathrm{min})$ for pseudo-first and pseudo-second-order, respectively. The values of $\mathrm{q}_{\mathrm{e}}, \mathrm{k}_{1}$, and $\mathrm{k}_{2}$ are determined from the slope and intercept values obtained from the plot which is drawn based on $\ln \left(\mathrm{q}_{\mathrm{e}}-\mathrm{q}_{\mathrm{t}}\right)$ versus $\mathrm{t}$ for the pseudo-first-order and $\mathrm{t} / \mathrm{q}_{\mathrm{t}}$ versus $\mathrm{t}$ for the pseudosecond-order. The experimental data fit pseudo-second-order reaction better than pseudo-firstorder based on the significance of the correlation coefficients (Figure 57). Rate constants for rare earths and thorium were found to be $1.21 / \mathrm{min}$ and $76.91 / \mathrm{min}$, respectively. On the other hand, $\mathrm{q}_{\mathrm{e}}$ values were calculated as 2.04 and $0.04 \mathrm{mg} / \mathrm{g}$, separately.
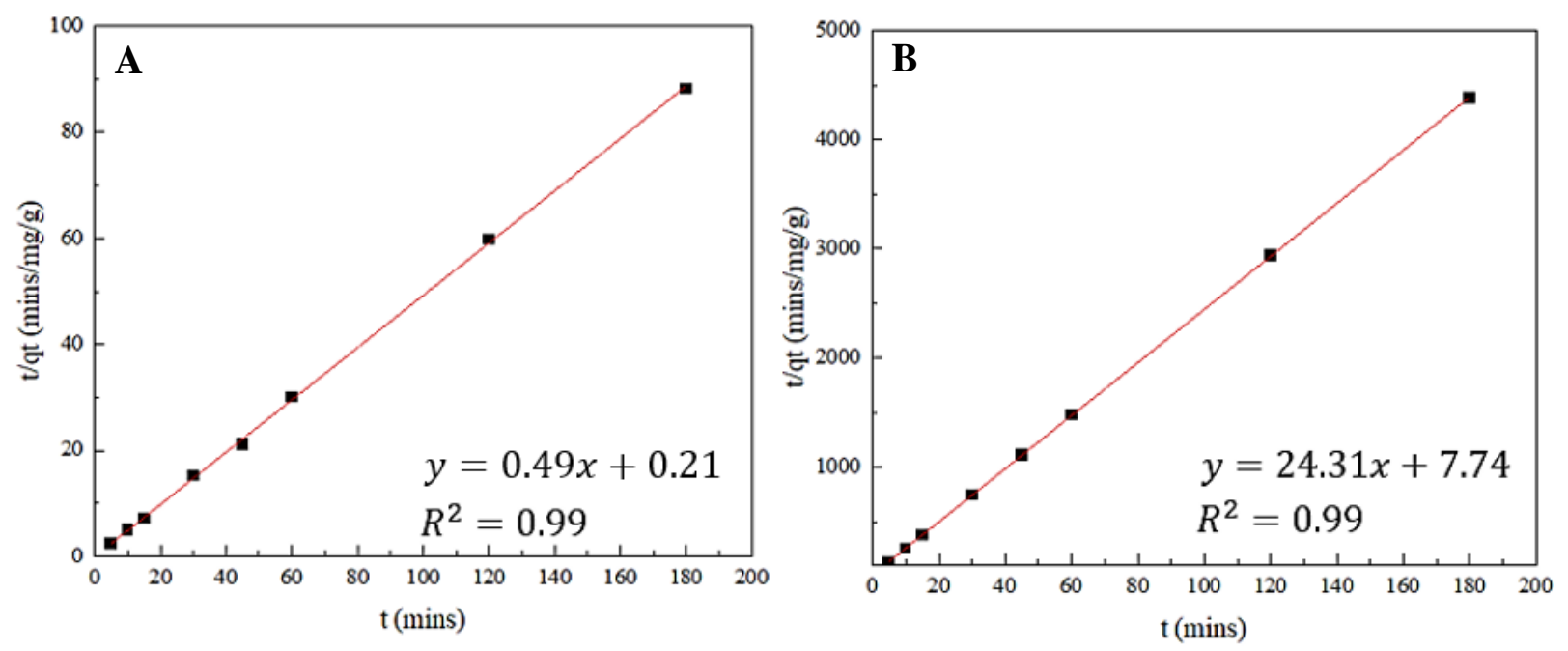

Figure 57 Pseudo-second-order reaction model for (A) total rare earths and (B) thorium.

Adsorption isotherms were subsequently developed based on the kinetic adsorption data. The isotherms indicate the distribution of molecules between the solid and liquid phases when the process reaches its equilibrium (Wang et al., 2012). Zhou et al. (2012) studied selective uranium adsorption using ion-imprinted magnetic chitosan. Their equilibrium data were fit to Langmuir's Isotherm equation which shows the monolayer adsorption process. Similarly, Li et al. (2018) studied thorium and uranium separation from rare earth elements by sorption on phenanthroline diamide functionalized graphene oxide, and their data well coincided with the Langmuir model. On the other hand, Kaynar et al. (2015) studied thorium removal by $\mathrm{ZnO}$, and their experimental data were best characterized by the Freundlich adsorption model. Based on previously conducted studies, Langmuir, Freundlich, and D-R isotherms, which aim to identify the adsorption 
mechanism underlying the experimental data, were decided on (Camacho et al., 2010; Li et al., 2012; Karimi et al., 2019).

The Langmuir isotherm assumes that the adsorption occurs at specific homogeneous sites within the adsorbents, and the energy of the adsorption is a constant (Langmuir, 1918). Moreover, according to Langmuir isotherm, adsorption occurs uniformly on the active sites of the sorbent, and once a sorbate occupies a site, no further sorption takes place at this site (Wang et al., 2012; Barkat et al., 2015). The Langmuir isotherm equation is represented as:

$$
q_{e}=\frac{q_{m} K_{L} C_{e}}{1+K_{L} C_{e}}
$$

Where $\mathrm{C}_{\mathrm{e}}$ is the equilibrium concentration $(\mathrm{mg} / \mathrm{L}), \mathrm{q}_{\mathrm{e}}$ is the amount absorbed at equilibrium $(\mathrm{mg} / \mathrm{g})$, $\mathrm{q}_{\mathrm{m}}$ is the Langmuir constant $(\mathrm{mg} / \mathrm{g}), \mathrm{K}_{\mathrm{L}}$ is constant for energy adsorption. Freundlich equation considers the adsorbent surface as heterogeneous (multilayer mode), and the amount absorbed at equilibrium is determined by the following equation (Liu et al., 2013):

$$
q_{e}=K_{F} C_{e}^{1 / n}
$$

Where $\mathrm{K}_{\mathrm{F}}$ is the Freundlich constant $(\mathrm{L} / \mathrm{g})$ and $\mathrm{n}$ is related to the adsorption density. D-R isotherm is governed by the equation given below. It does not assume that the materials have homogeneous surfaces (Barkat et al., 2015).

$$
\ln q_{e}=\ln q_{\max }-K_{a d s} \varepsilon^{2}
$$

Where $\mathrm{q}_{\max }$ is the capacity of saturation theory $(\mathrm{mg} / \mathrm{g}), \mathrm{K}_{\mathrm{ads}}$ is the adsorption energy constant $\left(\mathrm{mol}^{2} / \mathrm{kJ}\right)$, and $\varepsilon$ is the Polanyi potential which is defined as:

$$
\varepsilon=R T \log \left(1+\frac{1}{C_{e}}\right)
$$

where $\mathrm{R}$ is gas constant $(8.314 \mathrm{~J} / \mathrm{molK})$ and $\mathrm{T}$ is adsorption temperature $(\mathrm{K})$.

The Freundlich adsorption isotherm was found to be the best fit for both rare earths and thorium (Figure 58). The plots were generated following Eq. (37). Afterward, the slope and the intercept of the plots were used to calculate $1 / n$ and $K_{\mathrm{f}}$ values. The high correlation coefficient $\left(\mathrm{R}^{2}\right)$ observed in both isotherms suggests a good model fit. The $\mathrm{K}_{\mathrm{f}}$ values for rare earths and thorium were calculated as 2.05 and $0.24 \mathrm{~L} / \mathrm{g}$, respectively. A negative slope observed for rare earths and thorium indicates a reverse correlation between the amount adsorbed $\left(\mathrm{C}_{\mathrm{ads}}\right)$ and the equilibrium concentration $\left(\mathrm{C}_{\mathrm{e}}\right)$, which corresponded well with the experimental findings that the amount 
adsorbed decreased as time increased. Moreover, the Freundlich isotherm indicates multilayer adsorption rather than monolayer and considers a heterogeneous surface.
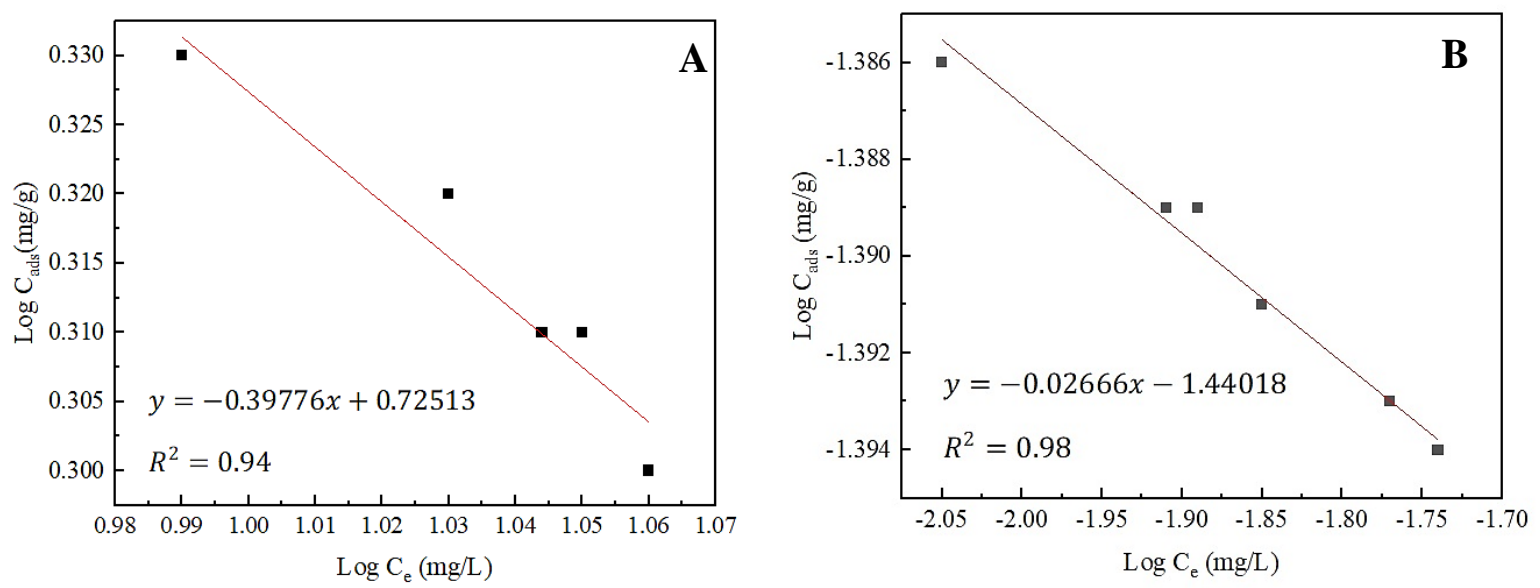

Figure 58 Freundlich adsorption isotherms of the elements onto zeolite. (A) total rare earths (B) thorium.

Dubinin-Raduskevich (D-R) adsorption isotherms of total rare earths and thorium are shown in Figure 59. The mean adsorption energy calculated from the isotherms reveals supplemental information on adsorption behavior. If the mean adsorption energy value falls between 1 and 8 $\mathrm{kJ} / \mathrm{mol}$, physical adsorption is considered. On the other hand, if the value varies between 9 and 16 $\mathrm{kJ} / \mathrm{mol}$, it represents chemical adsorption (Erden and Donat, 2016). The mean adsorption energy (E) values of rare earths and thorium were calculated as $5.8 \mathrm{~kJ} / \mathrm{mol}$ and $1.80 \times 10^{-3} \mathrm{~kJ} / \mathrm{mol}$, respectively. $\mathrm{E}<8 \mathrm{~kJ} / \mathrm{mol}$ indicates that both rare earth and thorium adsorption is governed by reversible physisorption and require less energy. Intermolecular forces are involved in accomplishing the adsorption. Moreover, the activity coefficient values were found to be $-1.48 \times 10^{6}$ $\mathrm{mol}^{2} / \mathrm{kJ}^{2}$ and $-1.54 \times 10^{-5} \mathrm{~mol}^{2} / \mathrm{kJ}^{2}$ for total rare earths and thorium, separately.
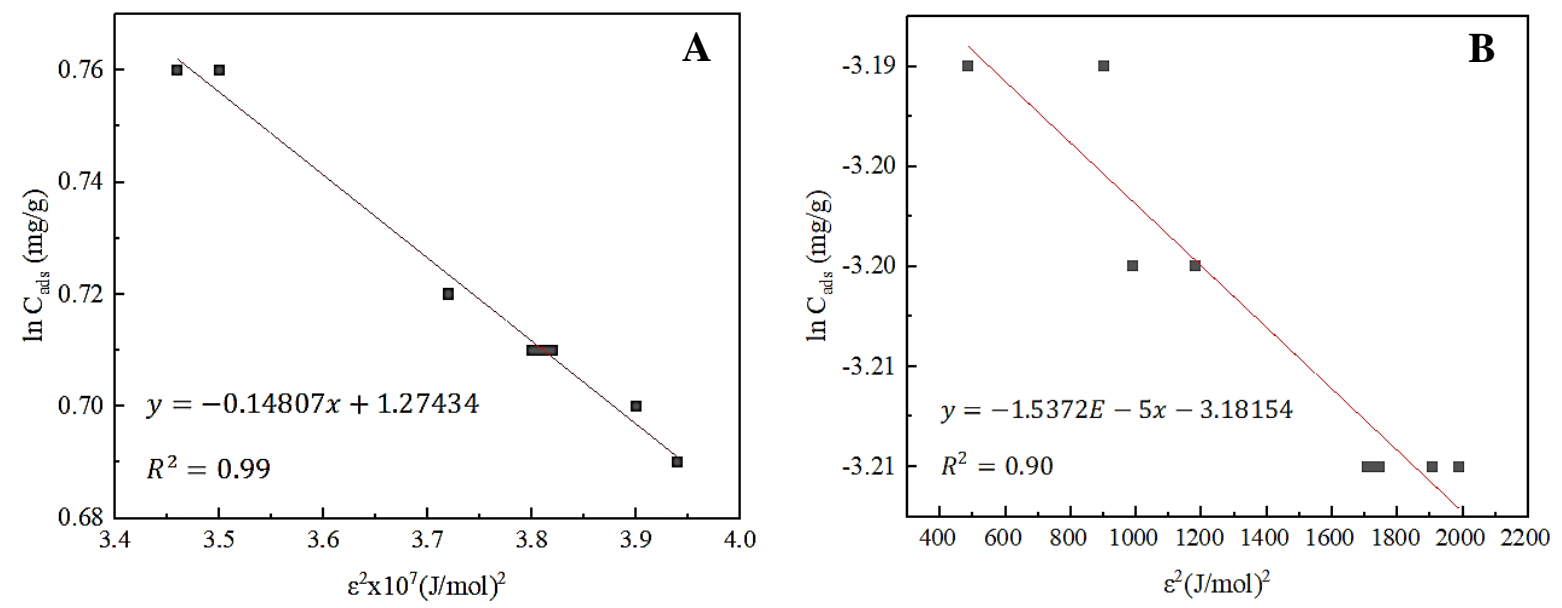

Figure 59 D-R adsorption isotherms of the elements onto zeolite. (A) total rare earths (B) thorium. 


\subsection{Conclusions}

In this chapter, zeolite adsorption was studied to remove thorium and uranium from rare earths. Experimental testing was carried out to evaluate the impact of four operational parameters, including solution $\mathrm{pH}$, zeolite particle size, zeolite amount, and contact time, on the adsorption recoveries. Following conclusions were reached at the end of the zeolite adsorption study:

1. It was observed that rare earths and uranium adsorption follow a similar pattern, which creates the separation challenge. The reason for this phenomenon can be the competition between rare earths and uranium ions.

2. As opposed to rare earth elements and uranium, test results indicate that zeolite adsorption is an effective technique for thorium removal.

3. Iron adsorption was in agreement with thorium adsorption and exhibited a steady-state adsorption under all operating conditions. Differently, a variation was observed for aluminum and magnesium depending on the test conditions.

4. The statistical model developed to predict the adsorption recovery of rare earths indicates that the initial solution $\mathrm{pH}$, particle size, and zeolite amount are the key operating parameters that all impacted the adsorption recovery of rare earths. However, the contact time was identified as an insignificant parameter.

5. Kinetic studies suggest a fast adsorption rate for thorium in contrast to total rare earths and uranium. Multilayer physisorption was determined for total rare earths and thorium when zeolite was selected as the adsorbent.

6. In conclusion, the best separation was achieved using 2.5 grams of $12-\mu \mathrm{m}$ zeolite at a solution $\mathrm{pH}$ of 3 for a contact period of 2 hrs. Under these conditions, almost $100 \mathrm{wt} \%$ thorium removal was achieved with approximately $20 \mathrm{wt} \%$ total rare earth loss. 


\section{CHAPTER 6}

\section{CONCEPTUAL PROCESS FLOWSHEET DEVELOPMENT}

Based on the above findings, a conceptual flowsheet was developed in order to produce high purity rare earth oxide while removing thorium and uranium (Figure 60). The proposed process flowsheet was developed using metallurgical simulation software, METSIM, and comprised of five main unit processes. The unit operations in the flowsheet are (1) leaching of the feedstock, (2) selective thorium removal by precipitation, (3) uranium removal by solvent extraction (4) rare earth oxalate precipitation, and (5) roasting for rare earth oxide production.

First, a solid coarse coal refuse sample is introduced to the leaching tank where the dissolution of the coarse coal refuse with acid at elevated temperature $\left(80^{\circ} \mathrm{C}\right)$ is achieved. After a sufficient retention time, the pregnant solution is subjected to filtration to remove the undissolved residue. A press filter is recommended due to the fine particle size. While the undissolved leach residue is sent to the tailings pond, the loaded leach solution is subjected to the second unit operation, where selective thorium removal is targeted via one-stage precipitation. Based on the experimental findings and literature review, it is recommended to keep the solution $\mathrm{pH}$ below 5 to prevent the loss of rare earth elements. When significant loss is observed, a re-dissolution-re-precipitation route can be followed to minimize the loss. According to the findings, there is no substantial difference between different alkaline salts; however, the use of sodium hydroxide $(\mathrm{NaOH})$ is more common than others. After the targeted $\mathrm{pH}$ is reached, the solution is sent to a second filter. Thorium is separated from the rest of the solution and sent to the same tailing pond with the leach residue. The material in this tailing pond can be utilized as mine backfilling since the concentration of thorium will be diluted by mixing it with other residues.

The solution remained from precipitation is treated with solvent extraction (the third unit process), where two-stage extraction and two-stage stripping are performed. In this study, the effect of scrubbing was found to be minimal; therefore, no scrubbing unit is proposed. After the solvent extraction unit, the loaded aqueous phase (uranium product stream) is sent to a pond, and the sulfuric acid used during the striping stage is recycled for reuse. The used extractant, TBP, is also sent to the recycling unit for further purification and reuse. To recycle the loaded organic phase, ammonium nitrate solution and deionized hot water can be used to remove undesirable elements, such as calcium, iron, magnesium, and phosphorus (Jorjani and Shahbazi, 2016).

On the other hand, the rare earth-containing aqueous stream is subjected to the fourth unit operation, where oxalic acid precipitation is performed to produce rare earth oxalates. The precipitated rare earth oxalates are filtered and subjected to roasting to convert them into sellable rare earth oxide products. The oxalic acid-containing stream is sent to another recycling system for its reuse.

The technical feasibility of this flowsheet was proven depending on the data obtained from laboratory and pilot plant scale samples Although this flowsheet is only including 
hydrometallurgical processing unit operations. Prior to this, several other unit operations, such as sample preparation (i.e., crushing, grinding, sizing), pre-treatment (i.e., density-based separation, froth flotation), pre-roasting, can be applied to increase the overall efficiency as well as to reduce the feed amount entering to the hydrometallurgical unit. For instance, significant increases were observed in the leaching of several coal samples by performing calcination before leaching. Due to high temperatures in the coal power plants, mineralogical changes may occur in the coal samples whose recovery can be enhanced with an additional calcination step prior to leaching by enhancing the liberation of particles. (Honaker et al., 2019). Even though the application of traditional mineral processing techniques alone for rare earth recovery from coal-based materials is ineffective, they can still be utilized as a pre-treatment method.

Although a techno-economic analysis has not been performed in this study, it is known that the majority of the cost belongs to the chemical reagents; therefore, recycling and reuse are essential. As Das et al. (2018) indicated in their study, the dosages of the chemical reagent and process time are two of the most important cost determiners. The researchers also mentioned that the economic viability of a process depends on the content of Scandium, too, due to the high market value. Furthermore, all water must be recycled as much as possible and fed into the process plant to reduce operational costs. However, since the developed flowsheet does not include any other unit operation than typical rare earth extraction processes, it is believed that no additional capital cost is needed. On the other hand, from an economic perspective, it was seen by the study of Honaker et al. (2019) a minimal plant size of 225 tph is required to generate profit.

Lastly, separated thorium and uranium have significant potential to be used in the fuel cycle or electricity production by nuclear energy. The U.S. Department of Energy estimates an approximately $28 \%$ increase in the need for electrical power from 2012 to 2040 (Nandanwar et al., 2016). On the other hand, uranium has been recognized as one of the critical minerals in the United States Geological Survey Bulletin. Aziman and Ismail (2020) have a thorough review study in which they investigated the sustainable recovery of thorium from the water leach residue of rare earth processing. Thorium has three to five times more potential reserves than uranium, and ${ }^{232} \mathrm{Th}$ can be converted to ${ }^{233} \mathrm{U}$ by absorbing slow neutrons (Kursun et al., 2018; Salehuddin et al., 2019). According to 2015 data, 174 million pounds of uranium (in the form of $\mathrm{U}_{3} \mathrm{O}_{8}$ ) was used in nuclear power plants worldwide, and a continuous increase is projected (Kim et al., 2016). The depleting uranium sources create a need for substitutes (Udayakumar et al., 2021). By converting thorium to uranium, thorium can effectively be used as a replacement for uranium-based nuclear reactors and can also become an alternative for uranium sources (Xiong et al., 2020). Therefore, if the standards are met, thorium and uranium separated from rare earth elements can create an additional production source. 


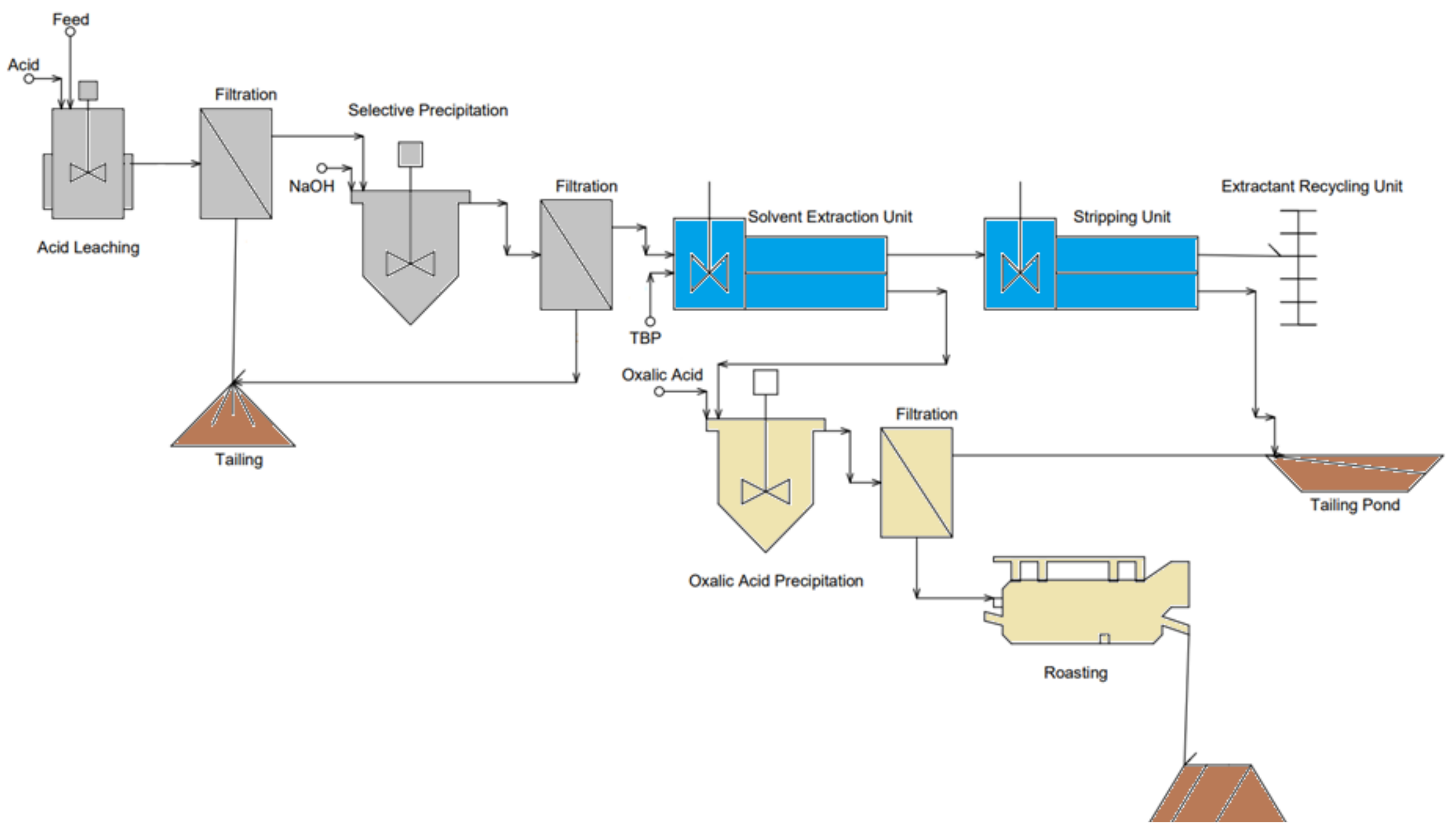

Figure 60 Conceptual flowsheet designed for the removal of thorium and uranium from rare earth elements 


\section{CHAPTER 7}

\section{CONCLUSIONS AND SUGGESTIONS FOR FUTURE STUDIES}

\subsection{Summary and Conclusions}

Over the past few decades, the demand for rare earth elements has increased rapidly and drastically. Their use has expanded tremendously as technology advances, and their importance in defense and military products is indisputable. Therefore, developing a supply chain for these elements from recycling or alternative sources has gained extreme importance. Given the global context, the identification of coal and coal by-products as potential rare earth sources is a novel finding and contribution. Considering the amounts of coal reserves, it possesses a vast potential to be a long-lasting source for rare earth elements. However, the association of thorium and uranium with rare earth minerals is an issue that requires additional treatment. Nonetheless, the studies on separating hazardous elements and rare earths from coal and coal byproducts are limited.

In that regard, this study was conducted to enlighten and provide insightful information on the removal of thorium and uranium from rare earth-containing solutions generated from coal coarse refuse. It was targeted to bridge the literature gap on REE-associated hazards in detail. This was accomplished through various hydrometallurgical separation processes considering the concentration of the feedstock sample. Three major separation techniques (i.e., precipitation, solvent extraction, and zeolite adsorption) were studied and evaluated. Initial exploratory tests were performed to identify the operational conditions. Selective precipitation was first assessed as an easy and cost-effective route. Later, solvent extraction was applied to further evaluate the separation potential. A modified experimental protocol incorporating both separation methods (i.e., selective precipitation and solvent extraction) was later developed and followed based on the results. Adsorption studies produced a good separation of thorium under all examined conditions. However, it did not generate sufficient separation between uranium and rare earth elements. A Box-Behnken design technique on the basis of response surface methodology was utilized for the experimental design of all examined separation techniques. Later, the statistical analysis sheds light on the process operating parameters that profoundly impact the removal of radioactive elements from rare earths. Finally, the findings were validated using a real system rare earth oxalate sample generated at the pilot processing plant located in Kentucky. As a result of all the research findings, a process flowsheet was developed to remove thorium and uranium and produce pure rare earth oxides.

Overall, the following conclusions were reached at the end of the study:

1. Experimental data obtained from initial selective precipitation and solvent extraction tests indicated that selective precipitation is effective in the removal of thorium. In contrast, solvent extraction has a superior performance in terms of selectively separating uranium from rare earths. 
2. It is possible to remove nearly $100 \%$ of thorium at a solution $\mathrm{pH}$ value of 4.85 with approximately $19.32 \%$ of rare earths and $47.98 \%$ of uranium co-precipitation.

3. Another separation technique needs to be utilized to further separate rare earth elements and uranium due to their overlapping precipitation $\mathrm{pH}$ regions.

4. An increase in the number of extraction stages proved to be more efficient than the single-stage solvent extraction process.

5. An experimental protocol incorporating both one-stage selective precipitation and twostage solvent extraction yielded an enhanced separation performance between thorium, uranium, and rare earth elements.

6. Based on the statistical analyses, among the examined operating parameters, extractant concentration, solvent extraction feed $\mathrm{pH}, \mathrm{O} / \mathrm{A}$ ratio played a significant role in the removal of uranium from rare earths.

7. As for the effect of the stripping agent, a noticeable reduction in uranium recovery was observed when sulfuric acid was used instead of DI water. However, the effect of sulfuric acid concentration on the overall separation was found as insignificant.

8. The optimum separation performance of the first subset tests following the modified experimental protocol was identified throughout the study, which corresponded to an overall rare earth, thorium, and uranium recovery of $80 \%, 0 \%$, and $3.1 \%$, separately. The optimum separation was obtained under the following operating condition: onestage precipitation at a $\mathrm{pH}$ value of 4.85 , solvent extraction feed $\mathrm{pH}$ of $3.5,50 \mathrm{v} \% \mathrm{TBP}$, $\mathrm{O} / \mathrm{A}$ ratio at $3: 1$.

9. Under the operating conditions of the second subset of solvent extraction tests, based on the overall experimental flowsheet, the recovery of rare earths into the REE product stream varied from $64.7 \%$ to $78.7 \%$, while the recovery of uranium into the same product steam fluctuated between $1.8 \%$ and $32.5 \%$ for the second subset of testing.

10. During the second subset, the best separation performance between the rare earths and uranium was achieved with $50 \mathrm{v} \% \mathrm{TBP}$, feed $\mathrm{pH}$ at 3.5, O: A ratio at 3, and $1 \mathrm{M} \mathrm{H}_{2} \mathrm{SO}_{4}$ as the strippant, which corresponded to an overall rare earth recovery of $73.4 \%$ and uranium recovery of $1.8 \%$.

11. Experimental findings of the adsorption studies suggested that rare earths and uranium adsorption follow a similar pattern, which creates the separation challenge. On the other hand, zeolite had a satisfactory capability for thorium removal.

12. The initial solution $\mathrm{pH}$, zeolite particle size, and zeolite amount were the key operating parameters that all impacted the adsorption recovery of rare earths.

13. Kinetic studies suggest a rapid adsorption rate for thorium in contrast to total rare earths and uranium. Multilayer physisorption was determined for total rare earths and thorium when zeolite was selected as the adsorbent.

14. Moreover, the experimental findings obtained so far have been supported by various characterization studies (e.g., XRD, XRF, SEM-EDX, BET, PSA) and fundamental investigations. 
15. At the end of all the studies, an effective process flowsheet was designed, which can be followed for the selective removal of thorium and uranium.

16. Following detailed parametric tests, performance validation tests were conducted on the rare earth oxalate sample produced from the pilot-scale processing facility. Test results validate the efficiency and reliability of the separation protocol developed.

In conclusion, considering the financial burden of disposing of coal waste and the environmental damage associated with it, this study has the utmost importance to utilize secondary sources to produce rare earth elements in an environmentally favorable manner. Specifically, this study had shown the possible separation strategies for thorium and uranium when a nontraditional feedstock was used. The separation behavior of the elements was systematically investigated in consideration of various aspects. As a result, the developed process can be applied to various feedstock materials. As mentioned in the previous chapters, the studies pertaining to the separation of hazardous elements and rare earths from coal and coal byproducts are limited. Given the lack of knowledge, experimental data, and commercialized technology in this aspect, it is believed that this study will contribute remarkably to both academia and the mining industry. Besides, considering the changes towards cleaner and more sustainable mining operations, this study has absolute significance and contribution to the field.

\subsection{Suggestions for Future Studies}

This study demonstrated the development of a process flowsheet that can effectively remove thorium and uranium from rare earth elements when non-traditional sources are utilized. However, from the standpoint of future commercialization, a detailed economic analysis of thorium and uranium removal from rare earth-bearing solutions should be conducted since there is no discussion on this subject yet.

Besides, the potential application of two novel separation methods, i.e., ionic liquid-based extraction and membrane-based separation, may be considered for future investigation. Unfortunately, only a few studies have been performed with ionic liquids. Ionic liquids are known as more environmentally friendly due to their high thermal stability and low volatility. On the other hand, membrane-based adsorption is superior to traditional adsorbents with high adsorption capacity.

Thorium and uranium purification can be studied to turn those separated from rare earths into the feedstock for the nuclear energy cycle. While the minimum required uranium (235U) concentration to be used in the fuel cycle spans between $0.7 \%$ to $5 \%$, a total of $1500 \mu \mathrm{g} / \mathrm{g}$ impurity (i.e., rare earth elements, $\mathrm{Al}, \mathrm{Fe}, \mathrm{Ca}, \mathrm{Si}, \mathrm{Pb}, \mathrm{Li}$, etc.) is acceptable. Therefore, the rare earth economic analysis can be expanded to consider thorium and uranium since they can be purified to be used in other industries for additional economic gains. 


\section{REFERENCES}

1. Abdel-Sabour, M. (2014). Uranium Fixation and Removal from Different Soil Types: Review. Journal of Nuclear Energy Science and Power Generation Technology.

2. Adiansyah, J., Rosano, M., Biswas, W., \& Haque, N. (2017). Life Cycle Cost Estimation and Environmental Valuation of Coal Mine Tailings Management. Journal of Sustainable Mining, 114-125.

3. Akdogan, G., \& Ghosh, T. (2014). Identification of REE in Some Alaskan Coal and Ash Samples. Mineral Industry Research Laboratory (MIRL) Institute of Northern Engineering, University of Alaska Fairbanks.

4. Akkaya, R. (2013). Uranium and Thorium Adsorption from Aqueous Solution Using A Novel Polyhydroxyethylmethacrylate-pumice Composite. Journal of Environmental Radioactivity, 58-63.

5. Aksoyoglu, S. (1989). Sorption of U(VI) on Granite. Journal of Radioanalytical and Nuclear Chemistry, 393-403.

6. Al-Areqi, W., Aniza, C., Bahri, C., Majid, A., \& Sarmani, S. (2016). Separation and Radiological Impact Assessment of Thorium in Malaysian Monazite Processing. Malaysian Journal of Analytical Sciences, 770-776.

7. Al-Areqi, W., Majid, A., \& Sarmani, S. (2014). Thorium, Uranium and Rare Earth Elements Content in Lanthanide Concentrate (LC) and Water Leach Purification (WLP) Residue of Lynas Advanced Materials Plant (LAMP). American Institute of Physics Conference Proceedings, (pp. 93-96).

8. Al-Areqi, W., Majid, A., Sarmani, S., \& Bahri, C. (2015). Thorium: Issues and Prospects in Malaysia. AIP Conference Proceedings (p. 1659). American Institute of Physics.

9. Alemrajabi, M., Rasmuson, A., Korkmaz, K., \& Forsberg, K. (2017). Recovery of Rare Earth Elements from Nitrophosphoric Acid Solutions. Hydrometallurgy, 253262.

10. Alex, P., Suri, A., \& Gupta, C. (1998). Processing of Xenotime Concentrate. Hydrometallurgy, 331-338.

11. Allegrini, E., Maresca, A., Olsson, M., Holtze, M., Boldrin , A., \& Astrup, T. (2014). Quantification of the Resource Recovery Potential of Municipial Solid Waste Incineration Bottom Ashes. Waste Management, 1627-1636.

12. Al-Nafai, I. (2015). Application of Pourbaix Diagrams in the Hydrometallurgical Processing of Bastnasite. M.S. Thesis, The Pennsylvania State University, Department of Materials Science and Engineering. 
13. Amaral, J., \& Morais, C. (2010). Thorium and Uranium Extraction from Rare Earth Elements in Monazite Sulfuric Acid Liquor Through Solvent Extraction. Minerals Engineering, 498-503.

14. Amer, T., Abdeall, W., Abdel Wahab, G., \& El-Sheikh, E. (2013). A Suggested Alternative Procedure for Processing of Monazite Mineral Concentrate. International Journal of Mineral Processing, 106-111.

15. American Coal Ash Association. (2017). 2016 Coal Combustion Product (CCP) Production and Use Survey Report. Farmington Hills, MI.

16. Anderson, C., Taylor, P., \& Anderson, C. (2017). Rare Earth Flotation Fundamentals: A Review. American Journal of Engineering Research, 155-166.

17. Anitha, M., Kotekar, M., Singh, D., Vijayalakshmi, R., \& Singh, H. (2014). Solvent Extraction Studies on Rare Earths from Chloride Medium with Organophosphorous Extractant Dinonyl Phenyl Phosphoric Acid. Hydrometallurgy, 128-132.

18. Anufrieva, A., Buinovskii, A., Guluyta, M., Molokov, P., Ageeva, L., \& Verkhoturova, V. (2014). Processing of REE-containing Ores and Concentrates. Procedia Chemistry, 119-125.

19. Arbuzov, S., Maslov, S., Finkelman, R., Mezhibor, A., Ilenok, S., Blokhin, M., \& Peregudina, E. (2018). Modes of Occurrence of Rare Earth Elements in Peat from Western Siberia. Journal of Geochemical Exploration, 40-48.

20. Asselin, G., Audrieth, L., \& Comings, E. (1949). The Separation of Thorium and Rare Erath Salts by Solvent Extraction. 640-648.

21. Ault, T., Krahn, S., \& Croff, A. (2015). Radiological Impacts and Regulations of Rare Earth Elements in Non-Nuclear Energy Production. Energies, 2066-2081.

22. Aziman, E., \& Ismail, A. (2020). Frontier Looking of Rare-Earth Processed Residue As Sustainable Thorium Resources: An Insight into Chemical Composition and Separation of Thorium. Progress in Nuclear Energy, 103471.

23. Badei, M., El-Naggar, I., El-Belihi, A., Aly, H., \& Aly, H. (1992). Sorption Behaviour of Uranium on Tin(IV) Antimonate from Aqueous Solutions. Radiochimica Acta, 89-92.

24. Balintova, M., \& Petrilakova, A. (2011). Study of pH Influence on Selective Precipitation of Heavy Metals from Acid Mine Drainage. Chemical Engineering Transactions, 345-350.

25. Banda, R., Jeon, H., \& Lee, M. (2012). Solvent Extraction Separation of Pr and Nd from Chloride Solution Containing La using Cyanex 272 and Its Mixture with Other Extractants. Separation and Purification Technology, 481-487. 
26. Barkat, M., Nibou, D., Amokrane, S., Chegrouche, S., \& Mellah, A. (2015). Uranium (VI) Adsorption on Synthesized 4A and P1 Zeolites: Equilibrium, Kinetic, and Thermodynamic Studies. Comptes Rendus Chimie, 261-269.

27. Batchu, N., Jeon, H., \& Lee, M. (2015). Solvent Extraction of Praseodymium (III) from Chloride Solutions by A Mixture of Cyanex 301 and LIX 63. Journal of Industrial and Engineering Chemistry, 286-290.

28. Baybas, D., \& Ulusoy, U. (2011). The Use of Polyacrylamide-Aluminosilicate Composites for Thorium Adsorption. Applied Clay Sciences, 138-146.

29. Belova, V., Egorova, N., Voshkin, A., \& Khol'kin, A. (2015). Extraction of Rare Earth Metals, Uranium and Thorium from Nitrate Solutions by Binary Extractants. Theoretical Foundations of Chemical Engineering, 545-549.

30. Bertetti, P., Pabalan, R., Pickett, D., \& Turner, D. (2011). Radionuclide Sorption Technical Assistance Activities at the Center for Nuclear Waste Regulatory Analyses. San Antonio, Texas.

31. Binnemans, K., Jones, P., Blanpain, B., Van Gerven, T., \& Pontikes, Y. (2015). Towards Zero-Waste Valorisation of Rare-Earth-Containing Industrial Process Residues: A Critical Review . Journal of Cleaner Production, 17-38.

32. Binnemans, K., Jones, P., Blanpain, B., Gerven, T., Yang, Y., Walton, A., \& Buchert, M. (2013). Recycling of Rare Earths: A Critical Review. Journal of Cleaner Production, 1-22.

33. Biswas, S., Pathak, P., Singh, D., \& Roy, S. (2013). Comparative Evaluation of Tri-n-butyl Phosphate (TBP) and Tris(2-Ethylhexyl) Phosphate (TEHP) for the Recovery of Uranium from Monazite Leach Solution. Separation Science and Technology, 2013-2019.

34. Blissett, R., Smalley, N., \& Rowson, N. (2014). An Investigation into Six Coal Fly Ashes from the United Kingdom and Poland to Evaluate Rare Earth Element Content. Fuel, 236-239.

35. Borai, E., El-Ghany, M., Ahmed, I., Hamed, M., El-Din, A., \& Aly, H. (2016). Modified Acidic Leachinh for Selective Separation of Thorium, Phosphate, and Rare Earth Concentrates from Egyptian Crude Monazite. International Journal of Mineral Processing, 34-41.

36. Brindley, G., \& Bastovanov, M. (1982). Clay and Clay Minerals, 135.

37. British Geological Survey. (2011). Rare Earth Elements.

38. Brookins, D. (1983). Eh-pH Diagrams for the Rare Earth Elements at 25C and one Bar Pressure. Geochemical Journal, 223-229.

39. Brookins, D. (1988). Eh-pH Diagrams for Geochemistry. Albuquerque: Springer-Verlag. 
40. Brown, C., \& Sherrington, L. (1979). Solvent Extraction used in Industrial Separation of Rare Earths. Journal of Chemical Technology and Biotechnology, 193209.

41. Camacho, L., Deng, S., \& Parra, R. (2010). Uranium Removal from Groundwater by Natural Clinoptilolite Zeolite: Effects of $\mathrm{pH}$ and Initial Feed Concentration. Journal of Hazardous Materials, 393-398.

42. Cheira, M., Atia, B., \& Kouraim, M. (2017). Uranium (VI) Recovery from Acidic Leach Liquor by Ambersep 920U SO4 Resin: Kinetic, Equilibrium and Thermodynamic Studies. Journal of Radiation Research and Applied Sciences, 307319.

43. Chellam, S., \& Clifford, D. (2002). Physical-Chemical Treatment of Groundwater Contaminated by Leachate from Surface Disposal of Uranium Tailings. Journal of Environmental Engineering, 942-952.

44. Chen, Y., Wei, Y., He, L., \& Tang, F. (2016). Separation of Thorium and Uranium in Nitric Acid Solution Using Silica Based Anion Exchange Resin. Journal of Chromatography A, 37-41.

45. Chu, S. (2011). Critical Materials Strategy. DIANE Pubslishing.

46. Chukanov, N., Blass, G., Pekov, I., Belakovskiy, D., Van, K., Rastsvetaeva, R., \& Aksenov, S. (2012). Perrierite-(La), (La, Ce, Ca)4(Fe2+, Mn)(Ti, Fe3+, Al)4(Si2O7)2O8, A New Species from the Eifel Volcanic District, Germany. Geology of Ore Deposits, 647-655.

47. Cooper, M. (2005). Naturally Occurring Radioactive Materials (NORM) in Australian Industries - Review of Current Inventories and Future Generation. Radiation Health and Safety Advisor Council.

48. Couper, J., Fair, J., Penney, W., \& Walas, S. (2009). Chemical Process Equipment: Selection and Design. Elsevier Science and Technology.

49. Dai, S., \& Finkelman, R. (2018). Coal As A Promising Source of Critical Elements: Progress and Future Prospects. International Journal of Coal Geology, 155164.

50. Dai, S., Seredin, V., Ward, C., Jiang, J., Hower, J., Song, X., . . Zhao, C. (2014). Composition and Modes of Occurrence of Minerals and Elements in Coal Combustion Products Derived from High-Ge Coals. International Journal of Coal Geology, 79-97.

51. Das, S., Gaustad, G., Sekar, A., \& Williams, E. (2018). Techno-Economic Analysis of Supercritical Extraction of Rare Earth Elements from Coal Ash. Journal of Cleaner Production.

52. Department of Energy. (2017). Report on Rare Earth Elements from Coal and Coal Byproducts. Washington DC. 
53. Dittrich, C., Kaya, S., Diekmann, F., \& Yagmurlu, B. (2016). Recovery of Rare Earth Elements and Scandium from European Deposits by Solvent Extraction. ALTA Uranium-REE Proceedings, (pp. 123-135).

54. Doi, K., Hirono, S., \& Sakamaki, Y. (1975). Uranium Mineralization by Ground Water in Sedimentary Rocks, Japan. Economic Geology, 628-646.

55. Dutta, T., Kim, K., Uchimiya, M., Kwon, E., Jeon, B., Deep, A., \& Yun, S. (2016). Global Demand for Rare Earth Resources and Strategies for Green Mining. Environmental Research, 182-190.

56. Dyer, A., \& Jozefowicz, L. (1992). The Removal of Thorium from Aqueous Solutions using Zeolites. Journal of Radioanalytical and Nuclear Chemistry, 47-62.

57. Ekmann, J. (2012). Rare Earth Elements in Coal Deposits - A Prospectivity Analysis. Search and Discovery.

58. Eskanazy, G. (1987). Rare Earth Elements and Yttrium in Lithotypes of Bulgarian Coals. Org. Geochem., 83-89.

59. Faghihian, H., Maragheh, M., Amini, M., \& Nezamzadeh, A. (Adsorption Science and Technology ). Thorium Ion Uptake by Zeolite A Synthesized from Natural Clinoptilolite Tuffs. 2004, 707-717.

60. Fasfous, I., \& Dawoud, J. (2012). Uranium(VI) Sorption by Multiwalled Carbon Nanotubes from Aqueous Solution. Applied Surface Science, 433-440.

61. Felmy, A., Rai, D., \& Mason, M. (1991). The Solubility of Hydrous Thorium(IV) Oxide in Chloride Media: Development of An Aqueous Ion-Interaction Model . Radiochimica Acta, 177-185.

62. Fiket, Z., Medunic, G., Turk, M., \& Kniewald, G. (2018). Rare Earth Elements in Superhigh-Organic-Sulfur Rasa Coal Ash (Croatia). International Journal of Coal Geology, 1-10.

63. Findeiß, M. (2016). Effects of Radioactive By-products Along the Extraction of Rare Earth Elements on Aquatic and Terrestrial Organisms. Aachen: Thesis.

64. Finkelman, R. (1999). Trace Elements in Coal Environmental and Health Significance. Biological Trace Element Research, 197-204.

65. Finkelman, R., Palmer, C., \& Wang, P. (2018). Quantification of the Modes of Occurrence of 42 Elements in Coal. International Journal of Coal Geology , 138-160.

66. Fouquet, Y., \& Martel-Jantin, B. (2014). Rare and Strategic Metals. In Y. Fouquet, \& D. Lacroix, Deep Marine Mineral Resources (pp. 55-64). Springer.

67. Gamboghi, J. (2015). Rare Earths: U.S. Geological Survey Mineral Commodity Summaries. 128-129. 
68. Gao, L., and Y. Chen. 2010. A study on the rare earth ore containing scandium by high-gradient magnetic separation. Journal of Rare Earths 25: 623-626.

69. Garcia-Tenorio, R., Manjon, G., Vioque, I., Jimenez-Ramos, M., Mantero, J., \& Diaz-Frances, I. (2018). Grey Monazite (Rare Earths) Mining in Centre of Spain: Characterization and Pre-operational Radiological Evaluation. Chemosphere, 691-697.

70. Garrels, R. (1955). Some Thermodynamic Relations Among the Uranium Oxides and Their Relation to the Oxidation States of the Uranium Ores of the Colorado Plateaus. Washington D.C.: U.S. Geological Survey.

71. Geier, R. (1979). Purex Process Solvent Literature Review. Washington.

72. Giri, R., \& Nath, G. (2016). Physicochemical Study of Extractants for Extraction of Rare Earth Element. Journal of Analytical Science and Technology.

73. Godelitsas, A., \& Armbruster, T. (2003). HEU-type Zeolites Modified by Transition Elements and Lead. Microporous and Mesoporous Materials, 3-24.

74. Gupta, B., Malik, P., \& Deep, A. (2002). Extraction of Uranium, Thorium and Lanthanides using Cyanex 923: Their Separations and Recovery from Monazite. Journal of Radioanalytical and Nuclear Chemistry, 451-456.

75. Habashi, F. (1997). Handbook of Extractive Metallurgy. Quebec: Wiley-VHC.

76. Hayes, S., Yu, P., O'Keefe, T., O'Keefe, M., \& Stoffer, J. (2002). The Phase Stability of Cerium Species in Aqueous Systems I. E-pH Diagram for the Ce-HCLO4H2O System. Journal of the Electrochemical Society, 623-630.

77. He, Y. (2014). Regulations of China's Rare Earth Production and Export. International Journal of Emerging Markets, 236-256.

78. Hedrick, J. (2000). Thorium. U.S. Geological Survey Minerals Yearbook.

79. Hidayah, N., \& Abidin, S. (2017). The Evolution of Mineral Processing in Extraction of Rare Earth Elements Using Solid-Liquid Extraction over Liquid-Liquid Extraction - A Review. Minerals Engineering, 103-113.

80. Honaker, R., Zhang, W., \& Werner, J. (2019). Acid Leaching of Rare Earth Elements from Coal and Coal Ash: Implications for Using Fluidized Bed Consumption to Assist in the Recovery of Critical Materials. Energy Fuels, 5971-5980.

81. Honaker, R., Groppo, J., Bhagavatula, A., Razaee, M., \& Zhang, W. (2016). Recovery of Rare Earth Minerals and Elements from Coal and Coal Byproducts. Louisville, KY.

82. Honaker, R., Hower, J., Eble, C., Weisenfluh, J., Groppo, J., Rezaee, M., \& Bhagavatula, A. (2014). Laboratory and Bench-Scale Testing for Rare Earth Elements, Final Project Report. 
83. Honaker, R., Zhang, W., Yang, X., \& Razaee, M. (2018). Conception of An Integrated Flowsheet for Rare Earth Elements Recovery from Coal Coarse Refuse. Minerals Engineering, 233-240.

84. Hostetler, P., \& Garrels, R. (1962). Transportation and Precipitation of Uranium and Vanadium at Low Temperatures with Special Reference to Sandstone-Type Uranium Deposits. Economic Geology and the Bulletin of the Society of Economic Geologists, 137-167.

85. Hower, J., Granite, E., Mayfield, D., Lewis, A., \& Finkelman, R. (2016). Notes on Contributions to the Science of Rare Earth Element Enrichment in Coal and Coal Combustion Byproducts. Minerals, 1-9.

86. Hu, J., Zheng, B., Finkelman, R., Wang, B., Wang, M., Li, S., \& Wu, D. (2006). Concentration and Distribution of Sixty-One Elements in Coals from DPR Korea. Fuel, 679-688.

87. Hua, Z., Wang, J., Wang, L., Zhao, Z., Li, X., Xiao, Y., \& Yang, Y. (2014). Selective Extraction of Rare Earth Elements from NdFeB Scrap by Molten Chlorides . ACS Sustain. Chem. Eng., 2536-2543.

88. Humelnicu, D., Drochioiu, G., Sturza, M., Cecal, A., \& Popa, K. (2006). Kinetic and Thermodynamic Aspects of U(VI) and Th(IV) Sorption on A Zeolitic Volcanic Tuff. Journal of Radioanalytical and Nuclear Chemistry , 637-640.

89. Huang, Q., Noble, A., Herbst, J., \& Honaker, R. (2018). Liberation and Release of Rare Earth Minerals from Middle Kittanning, Fire Clay, and West Kentucky No. 13 Coal Sources. Powder Technology, 242-252.

90. Huang, Q., Talan, D., Restrepo, J., Baena, O., Kecojevic, V., \& Noble, A. (2019). Characterization Study of Rare Earths, Yttrium, and Scandium from Various Colombian Coal Samples and Non-Coal Lithologies. International Journal of Coal Geology, 14-26.

91. Hughes, K., \& Singh, R. (1980). The Isolation of Thorium from Monazite by Solvent Extraction. Hydrometallurgy, 25-33.

92. Hvistendahl, M. (2007, December 13). Coal Ash Is More Radioactive Than Nuclear Waste. Retrieved from Scientific American: https://www.scientificamerican.com/article/coal-ash-is-more-radioactive-thannuclear-waste/

93. International Atomic Energy Agency. (2000). Recycle and Reuse of Materials and Components from Waste Streams of Nuclear Fuel Cycle Facilities. Vienna.

94. Jaffary, N., Khoo, K., Mohamed, N., Yusof, M., \& Fadzil, S. (2019). Malaysian Monazite and Its Processing Residue: Chemical Composition and Radioactivity. Journal of Radioanalytical and Nuclear Chemistry, https://doi.org/10.1007/s10967019-06813-1. 
95. Jain, V., Handa, A., Sait, S., Shrivastav, P., \& Agrawal, Y. (2001). Preconcentration, Separation and Trace Determination of Lanthanum(III), Cerium(III), Thorium(IV) and Uranium(VI) on Polymer Supported o-Vanillinsemicarbazone. Analytica Chimica Acta, 237-246.

96. Jakobsson, L., Kennedy, M., Aune, R., \& Tranell, G. (2016). Recovery of Rare Earth Elements from the Ferrous Fraction of Electronic Waste, REWAS 2016: Towards Materials Resource Sustainability . Springer.

97. Jha, M., Kumari, A., Panda, R., Kumar, J., Yoo, K., \& Lee, J. (2016). Review on Hydrometallurgical Recovery of Rare Earth Metals. Hydrometallurgy, 2-26.

98. Jiabao, L., \& Jie, L. (2009, 09 07). Rare Earth Industry Adjusts to Slow Market. Retrieved from China Daily : http://www.chinadaily.com.cn/bw/200909/07/content_8660849.htm

99. Jordens, A. (2016). The Beneficiation of Rare Earth Element-Bearing Minerals. Montreal: Thesis, McGill University.

100. Jorgensen, S. (1989). Chapter 4-Adsorption and Ion Exchange. Developments in Environmental Modelling, 65-81.

101. Jorjani, E., \& Shahbazi, M. (2016). The Production of Rare Earth Elements Group via Tributyl Phosphate Extraction and Precipitation Stripping Using Oxalic Acid. Arabian Journal of Chemistry, 1532-1539.

102. Jun, L., Zhenggui, W., Deqian, L., Gengxiang, M., \& Zucheng, J. (1998). Recovery of Ce (IV) and Th (IV) from Rare Earths (III) with Cyanex 923. Hydrometallurgy, 77-87.

103. Jyothi, R., Thenepalli, T., Ahn, J., Parhi, P., Chung, K., \& Lee, J. (2020). Review of Rare Earth Elements Recovery from Secondary Resources for Clean Energy Technologies: Grand Opportunities to Create Wealth from Waste. Journal of Cleaner Production, 122048.

104. Kammerer, J., Carle, R., \& Kammerer, D. (2011). Adsorption and Ion Exchange: Basic Principles and Their Application in Food Processing. Journal of Agricultural and Food Chemistry Review, 22-42.

105. Karayigit, A., Gayer, R., Querol, X., \& Onacak, T. (2000). Contents of Major and Trace Elements in Feed Coals from Turkish Coal-Fired Power Plants. International Journal of Coal Geology, 169-184.

106. Karimi, S., Yaraki, M., \& Karri, R. (2019). A Comprehensive Review of the Adsorption Mechanisms and Factors Influencing the Adsorption Process from the Perspective of Bioethanol Dehydration. Renewable and Sustainable Energy Reviews, 535-553.

107. Kaygun, A., \& Akyil, S. (2007). Study of the Behaviour of Thorium Adsorption on PAN/Zeolite Composite Adsorbent. Journal of Hazardous Materials, 357-362. 
108. Kaynar, U., Ayvacikli, M., Hicsonmez, U., \& Kaynar, S. (2015). Removal of Thorium(IV) Ions from Aqueous Solutions by A Novel Nanoporous ZnO: Isotherms, Kinetic and Thermodynamic Studies. Journal of Environmental Radioactivity, 145151.

109. Ketris, M., \& Yudovich, Y. (2009). Estimations of Clarkes for Carbonaceous Biolithes: World Averages for Trace Element Contents in Black Shales and Coals. International Journal of Coal Geology, 135-148.

110. Khalili, F., \& Al-Banna, G. (2015). Adsorption of Uranium(VI) and Thorium(IV) by insolubilized Humic Acid from Ajloun Soil - Jordan. Journal of Environmental Radioactivity, 16-26.

111. Kilincarslan, A., \& Akyil, S. (2005). Uranium Adsorption Characteristic and Thermodynamic Behavior of Clinoptilolite Zeolite. Journal of Radioanalytical and Nuclear Chemistry, 541-548.

112. Kilislioglu, A., \& Bilgin, B. (2003). Thermodynamic and Kinetic Investigations of Uranium Adsorption on Amberlite IR-118H Resin. Applied Radiation and Isotopes, 155-160.

113. Kim, E., \& Osseo-Asare, K. (2012). Aqueous Stability of Thorium and Rare Earth Metals in Monazite Hydrometallurgy: Eh-pH Diagrams for the Systems T-, Ce-, La-, Nd-, (PO4)-(SO4)-H2O at 25C. Hydrometallurgy, 67-78.

114. Kim, H., Eggert, R., Carlsen, B., \& Dixon, B. (2016). Potential Uranium Supply from Phosphoric Acid: A U.S Analysis Comparing Solvent Extraction and Ion Exchange Recovery. Resources Policy, 222-231.

115. King, H. (2020, 11 4). REE-Rare Earth Elements and Their Uses. Retrieved from Geology: https://geology.com/articles/rare-earth-elements/

116. Kini, G. (2006). Characterization of Synthetic Zeolites Optimized for Heavy Metal Uptake from Wastewater. Thesis, University of Florida.

117. Kolker, A., Scott, C., Hower, J., Vazquez, J., Lopano, C., \& Dai, S. (2017). Distribution of Rare Earth Elements in Coal Combustion Fly Ash, Determined by SHRIMP-RG Ion Microprobe. International Journal of Coal Geology, 1-10.

118. Kuang, S., Zhang, Z., Li, Y., Wei, H., \& Liao, W. (2017). Synergistic Extraction and Separation of Rare Earths from Chloride Medium by the Mixture of HEHAPP and D2EHPA. Hydrometallurgy, 78-83.

119. Kursun, I., Tombal, T., \& Terzi, M. (2018). Solubility of Eskisehir Thorium/Rare Earth Ores in Sulphuric and Nitric Acids. Physicochemical Problems of Mineral Processing, 476-483.

120. Kutahyali, C., \& Eral, M. (2010). Sorption Studies of Uranium and Thorium on Activated Carbon Prepared from Olive Stones: Kinetic and Thermodynamic Aspects. Journal of Nuclear Materials, 251-256. 
121. Kuzmin, V., Pashkov, G., Lomaev, V., Voskresenskaya, E., \& Kuzmina, V. (2012). Combined Approaches for Comprehensive Processing of Rare Earth Metal Ores. Hydrometallurgy, 1-6.

122. Ladeira, A., \& Goncalves, C. (2007). Influence of Anion Species on Uranium Separation from Acid Mine Water Using Strong Base Resins. Journal of Hazardous Materials, 499-504.

123. Lange, C., Camargo, I., Figueiredo, A., Castro, L., Vasconcellos, M., \& Ticianelli, R. (2017). A Brazilian Coal Fly Ash As A Potential Source for Rare Earth Elements. Journal of Radioanal Nucl. Chem., 1235-1241.

124. Langmuir, D. (1978). Uranium Solution-Mineral Equilibria at Low Temperatures with Applications to Sedimentary Ore Deposits. Geohemica et Cosmochimica Acta, 547-569.

125. Langmuir, I. (1918). The Adsorption of Gases on Plane Surfaces of Glass, Mica and Platinum. American Chemical Society, 1361-1368.

126. Lanzerstorfer, C. (2018). Pre-processing of Coal Combustion Fly Ash by Classification for Enrichment of Rare Earth Elements. Energy Reports, 660-663.

127. Li, F., Yang, Z., Weng, H., Chen, G., Lin, M., \& Zhao, C. (2018). High Efficient Separation of U(VI) and Th(IV) from Rare Earth Elements in Strong Acidic Solution by Selective Sorption on Phenanthroline Diamide Functionalized Graphene Oxide. Chemical Engineering Journal, 340-350.

128. Li, Z., Chen, F., Yuan, L., Liu, Y., Zhao, Y., Chai, Z., \& Shi, W. (2012). Uranium(VI) Adsorption on Graphene Oxide Nanosheets from Aqueous Solutions. Chemical Engineering Journal, 539-546.

129. Lin, R., Soong, Y., \& Granite, E. (2018). Evaluation of Trace Elements in U.S. Coals Using the USGS COALQUAL Database Version 3.0. Part I: Rare Earth Elements and Yttrium (REY). International Journal of Coal Geology, 1-13.

130. Liu, H., \& Pan, Z. (2011). NORM Situation in Non-Uranium Mining in China. Annals of the ICRP, 343-351.

131. Liu, X., Li, J., Wang, X., Chen, C., \& Wang, X. (2015). High Performance of Phosphate Functionalized Graphene Oxide for the Selective Adsorption of U(VI) from Acidic Solution. J. Nucl. Mater., 56-64.

132. Liu, Y., Wang, Y., Zhang, Z., Cao, X., Nie, W., Li, Q., \& Hua, R. (2013). Removal of Uranium from Aqueous Solution by A Low Cost and High-Efficient Adsorbent. Applied Surface Science, 68-74.

133. Lu, Y., Wei, H., Zhang, Z., Li, Y., Wu, G., \& Liao, W. (2016). Selective Extraction and Separation of Thorium from Rare Earths by A Phosphorodiamidate Extractant. Hydrometallurgy, 192-197. 
134. Malamis, S., \& Katsou, E. (2013). A Review on Zinc and Nickel Adsorption on Natural and Modified Zeolite, Bentonite, and Vermiculite: Examination of Process Parameters, Kinetics and Isotherms. Journal of Hazardous Materials, 428-461.

135. McLellan, B., Corder, G., Golev, A., \& Ali, S. (2014). Sustainability of the Rare Earths Industry. Procedia Environmental Sciences, 280-287.

136. Menzies, I., \& Rigby, F. (1961). Separation of Thorium from Uranium and Rare Earth Elements by Solvent Extraction with Tri-n-Butyl Phosphate-Xylene. J. Appl. Chem., 104-113.

137. Merritt, R. R. (1990). High temperature methods for processing monazite: I. Reaction with calcium chloride and calcium carbonate. Journal of the Less Common Metals, 166(2), $197-210$.

138. Metaxas, M., Kasselouri-Rigopoulou, V., Galiatsatou, P., Konstantopoulou, C., \& Oikonomou, D. (2003). Thorium Removal by Different Adsorbents. Journal of Hazardous Materials, 71-82.

139. Michieka, N. (2017, June). Rare Earth Elements and Their Role in Renewable Energy. Retrieved from https://www.bakersfield.com/kern-business-journal/rare-earthelements-and-their-role-in-renewable-energy/article_2dff98b6-8b4e-5ecc-91f6ef $18 \mathrm{c} 0 \mathrm{a} 2 \mathrm{c} 44 \mathrm{e} \cdot \mathrm{html}$

140. Milonjic, S., Cokesa, D., \& Stevanovic, R. (1992). Dynamic Adsorption of Uranium(VI) and Zirconium(IV) on Silica Gel. Journal of Radioanalytical and Nuclear Chemistry, 79-90.

141. Misaelides, P., Godelitsas, A., Filippidis, A., Charistos, D., \& Anousis, I. (1995). Thorium and Uranium Uptake by Natural Zeolitic Materials. The Science of the Total Environment, 237-246.

142. Monhemius, J. (1977). Precipitation Diagrams for Metal Hydroxides, Sulfides, Arsenates and Phosphates. Transactions Institution of Mining and Metallurgy, 202-206.

143. Monji, A., Ghoulipour, V., \& Mallah, M. (2016). Selective Sorption of Uranium (IV) from Hydrochloric Acid Media by Agro-Industrial Byproducts. Annals of Nuclear Energy, 115-121.

144. Motsi, T., Rowson, N., \& Simmons, M. (2009). Adsorption of Heavy Metals from Acid Mine Drainage by Natural Zeolite. Int. J. Miner. Process., 42-48.

145. Muhling, J., Suvorova, A., \& Rasmussen, B. (2014). The Occurrence and Composition of Chevkinite-(Ce) and Perrierite-(Ce) in Theoleitic Intrusive Rocks and Lunar Mare Basalt. American Mineralogist, 1911-1921.

146. Nandanwar, S., Coldsnow, K., Utgikar, V., Sabharwall, P., \& Aston, D. (2016). Capture of Harmful Radioactive Contaminants from Off-Gas Stream Using Porous 
Solid Sorbents for Clean Environment - A Review. Chemical Engineering Journal, 369-381.

147. Nasab, M. (2014). Solvent Extraction Separation of Uranium (VI) and Thorium (IV) with Neutral Organophosphorus and Amine Ligands. Fuel, 595-600.

148. Nasab, M., Sam, A., \& Milani, S. (2011). Determination of Optimum Process Conditions for the Separation of Thorium and Rare Earth Elements by Solvent Extraction. Hydrometallurgy, 141-147.

149. Navarro, J., \& Zhao, F. (2014). Life-Cycle Assessment of the Production of Rare Earth Elements for Energy Applications: A Review. Retrieved from Frontiers in Energy Research: https://www.frontiersin.org/articles/10.3389/fenrg.2014.00045/full

150. Navrotsky, A., Shvareva, T., \& Guo, X. (2013). Thermodynamics of Uranium Minerals and Related Materials. In Mineralogical Association of Canada Short Course 43. Winnipeg, MB.

151. Obaid, S., Gaikwad, D., Sayyed, M., Al-Rashdi, K., \& Pawar, P. (2018). Heavy Metal Ions Removal from Waste Water by the Natural Zeolites. Materials Today: Proceedings (pp. 17930-17934). Elsevier.

152. Omodara, L., Pitkaaho, S., Turpeinen, E., Saavalainen, P., Oravisjarvi, K., \& Keiski, R. (2019). Recycling and Substitution of Light Rare Earth Elements, Cerium, Lanthanum, Neodymium, and Praseodymium from End-of-Life Applications- A Review. Journal of Cleaner Production , 117573.

153. Ozbayoglu, G., \& Atalay, M. (2000). Beneficiation of Bastnaesite by A MultiGravity Separator. Journal of Alloys and Compounds, 520-523.

154. Palmieri, M. (1987). Separation and Determination of Trace Metal Ions Using Organic Chelating Reagents. Thesis, Iowa State University.

155. Pan, J., Zhou, C., Tang, M., Cao, S., Liu, C., Zhang, N., . . Ji, W. (2019). Study on the Modes of Occurrence of Rare Earth Elements in Coal Fly Ash by Statistics and A Sequential Chemical Extraction Process. Fuel, 555-565.

156. Panda, R., Kumari, A., Jha, M., Hait, J., Kumar, V., Kumar, J., \& Lee, J. (2014). Leaching of Rare Earth Metals (REMs) from Korean Monazite Concentrate. Journal of Industrial and Engineering Chemistry, 2035-2042.

157. Papastefanou, C. (2007). Radioactivity of Coals and Fly Ashes. Journal of Radioanalytical and Nuclear Chemistry, 29-35.

158. Parzentny, H., \& Rog, L. (2019). The Role of Mineral Matter in Concentrating Uranium and Thorium in Coal and Combustion Residues from Power Plants in Poland. Minerals, doi:10.3390/min9050312.

159. Paul, A., \& Pillai, K. (1991). Natural Radionuclides in A Tropical River Subjected to Pollution. Water, Air and Soil Pollution, 305-319. 
160. Paul, J., \& Campbell, G. (2011). Investigating Rare Earth Element Mine Development in EPA Region 8 and Potential Environmental Impacts. The United States Environmental Protection Agency.

161. Paulick, H., \& Machacek, E. (2017). The Global Rare Earth Element Exploration Boom: An Analysis of Resources Outside of China and Discussion of Development Perspectives. Resources Policy, 134-153.

162. Pillai, P. (2007). Naturally Occurring Radioactive Materials (NORM) in the Extraction and Processing of Rare Earths. Proceedings of An International Symposium (pp. 197-221). Seville, Spain: International Atomic Energy Agency.

163. Ponou, J., Dodbiba, G., Anh, J., \& Fujita, T. (2016). Selective Recovery of Rare Earth Elements from Aqueous Solution Obtained from Coal Power Plant Ash. Journal of Environmental Chemical Engineering, 3761-3766.

164. Pourbaix, M. (1974). Atlas of Electrochemical Equilibria in Aqueous Solutions. Brussels: Cebelcor.

165. Peiro, L., \& Mendez, G. (2013). Material and Energy Requirement for Rare Earth Production. JOM, 1327-1340.

166. Qadeer, R., Hanif, J., Saleem, M., \& Afzal, M. (1992). Effect of Alkali Metals, Alkaline Earth Metals and Lanthanides on the Adsorption of Uranium on Activated Charcoal from Aqueous Solutions. Journal of Radioanalytical and Nuclear Chemistry, 243-253.

167. Qi, D. (2018). Extractants Used in Solvent Extraction-Separation of Rare Earths: Extraction Mechanism, Properties, and Features. In Hydrometallurgy of Rare Earths-Separation and Extraction (pp. 187-389).

168. Qin, S., Lu, Q., Li, Y., Wang, J., Zhao, Q., \& Gao, K. (2018). Relationships Between Trace Elements and Organic Matter in Coal. Journal of Geochemical Exploration, 101-110.

169. Quinn, J., Soldenhoff, K., Stevens, G., \& Lengkeek, N. (2015). Solvent Extraction of Rare Earth Elements Using Phosphonic/Phosphinic Acid Mixtures. Hydrometallurgy, 298-305.

170. Rabie, K., Sayed, S., Lasheen, T., \& Salama, I. (2007). Europium Separation from A Middle Rare Earths Concentrate Derived from Egyptian Black Sand Monazite. Hydrometallurgy, 121-130.

171. Radioactive Elements in Coal and Fly Ash: Abundance, Forms, and Environmental Significance. (1997, October). Retrieved from United States Geological Survey: https://pubs.usgs.gov/fs/1997/fs163-97/FS-163-97.html

172. Ramesh, K., Reddy, K., \& Nandanan, R. (2014). Porosity Distribution, Surface Area and Morphology of Synthetic Potassium Zeolites: A SEM and N2 Adsorption Study. Communications in Soil Science and Plant Analysis. 
173. Rand, M., Fuger, J., Grenthe, I., Neck, V., \& Rai, D. (2008). Chemical Thermodynamics of Thorium. Oxford.

174. Regenspurg, S., Margot-Roquier, C., Harfouche, M., Froidevaux, P., Steinmann, P., Junier, P., \& Bernier-Latmani, R. (2010). Speciation of NaturallyAccumulated Uranium In An Organic-Rich Soil of An Alpine Region (Switzerland). Geochimica et Cosmochimica Acta , 2082-2098.

175. Rim, K., Koo, K., \& Park, J. (2013). Toxicological Evaluations of Rare Earths and Their Health Impacts to Workers: A Literature Review. Saf. Health Work, 12-26.

176. Rozmaric, M., Ivsic, A., \& Grahek, Z. (2009). Determination of Uranium and Thorium in Complex Samples Using Chromatographic Separation, ICP-MS and Spectrophotometric Detection. Talanta, 352-362.

177. Salehuddin, A., Ismail, A., Bahri, C., \& Aziman, E. (2019). Economic Analysis of Thorium Extraction from Monazite. Nuclear Engineering and Technology, 631-640.

178. Samsonov, M., Trofimov, T., Kulyako, Y., Vinokurov, S., Malikov, D., Batorshin, G., \& Myasoedov, B. (2015). Recovery of Rare Earth Elements, Uranium and Thorium from Monazite Concentrate by Supercritical Fluid Extraction. Radiochemistry, 343-347.

179. Sani, A., Bandegharaei, A., Hosseini, S., Kharghani, K., Zarei, H., \& Rastegar, A. (2015). Kinetic, Equilibrium and Thermodynamic Studies on Sorption of Uranium and Thorium from Aqueous Solutions by A Selective Impregnated Resin Containing Carminic Acid. Journal of Hazardous Materials, 152-163.

180. Schreiber, A., Marx, J., Zapp, P., Hake, J., Vobenkaul, D., \& Friedrich, B. (2016). Environmental Impacts of Rare Earth Mining and Separation Based on Eudialyte: A New European Way. Resources, doi:10.3390/resources5040032.

181. Schuler, D., Buchert, M., Liu, R., Dittrich , S., \& Merz, C. (2011). Study on Rare Earths and Their Recycling. Darmstadt.

182. Seo, E., Cheong, Y., Yim, G., Min, K., \& Geroni, J. (2017). Recovery of Fe, Al and Mn in Acid Coal Mine Drainage by Sequential Selective Precipitation with Control of pH. Catena, 11-16.

183. Seredin, V. (1996). Rare Earth Element-Bearing Coals from the Russian Far East Deposit. International Journal of Coal Geology, 101-129.

184. Seredin, V., \& Dai, S. (2012). Coal Deposits as Potential Alternative Sources for Lanthanides and Yttrium. International Journal of Coal Geology, 67-93.

185. Sikalidis, C., Alexiades, C., \& Misaelides, P. (2008). Adsorption of Uranium and Thorium from Aqueous Solutions by the Clay Minerals Montmorillonite and Vermiculite. Toxicological and Environmental Chemistry, 175-180. 
186. Silva, R. G., De Morais, C. A., \& Teixeria, L. V. (2018). Selective Removal of Impurities from Rare Earth Sulphuric Liquor Using Different Reagents. Minerals Engineering, 238-246.

187. Song, G., Yuan, W., Zhu, X., Wang, X., Zhang, C., Li, J., \& Wang, J. (2017). Improvement in Rare Earth Element Recovery from Waste Thrichromatic Phosphors by Mechanical Activation. J. Clean. Prod., 361-370.

188. Song, Y., Wang, Y., Wang, L., Song, C., Yang, Z., \& Zhao, A. (1999). Recovery of Uranium from Carbonate Solutions Using Strongly Basic Anion Exchanger 4. Column Operation and Quantitative Analysis. Reactive and Functional Polymers, 245252.

189. Srinivas, C., Venkatesh, K., Wattal, P., Theyyunni, T., Kartha, P., \& Tripathi, S. (1994). Alkaline Hydrolysis Process for Treatment and Disposal of Purex Solvent Waste. Bombay, India: Bhabha Atomic Research Centre.

190. Suli, L., Ibrahim, W., Aziz, B., Deraman, M., \& Ismail, N. (2017). A Review of Rare Earth Mineral Processing Technology. Chemical Engineering Research Bulletin, 20-35.

191. Thakur, N. (2000). Separation of Rare Earths by Solvent Extraction . Mineral Processing and Extractive Metallurgy Review, 277-306.

192. Thommes, M., Kaneko, K., Niemark, A., Olivier , J., Rodriguez-Reinoso, F., Rouquerol, J., \& Sing, K. (2015). Physisoprtion of Gases, With Special Reference to the Evaluation of Surface Area and Pore Size Distribution. Pure Applied Chemistry.

193. Tomazic, B., Branica, M., \& Tezak, B. (1962). Precipitation and Hydrolysis of Uranium(VI) in Aqueous Solutions: Uranyl Nitrate-Potassium Hydroxide-Neutral Electrolyte. Croatica Chemica Acta, 41-50.

194. Torapava, N., Persson, I., Eriksson, L., \& Lundberg, D. (2009). Hydration and Hydrolysis of Thorium (IV) in Aqueous Solution and the Structures of Two Crystalline Throium(IV) Hydrates. Inorganic Chemistry, 11712-11723.

195. U.S. Department of Energy. (2011). Critical Materials Strategy.

196. U.S. Environmental Protection Agency. (1999). Understanding Variation in Partition Coefficient, Kd, Values, Volume II: Review of Geochemistry and Available Kd Values for Cadmium, Cesium, Chromium, Lead, Plutonium, Radon, Strontium, Thorium, Tritium, and Uranium. Washington, DC: U.S. Environmental Protection Agency and U.S. Department of Energy.

197. Uda, T., Jacob, K., \& Hirasawa, M. (2000). Technique for Enhanced Rare Earth Separation. Science, 2326-2329.

198. United States Geological Survey. (1997). Retrieved from Radioactive Elements in Coal and Fly Ash: Abundance, Forms, and Environmental Significance: https://pubs.usgs.gov/fs/1997/fs163-97/FS-163-97.html 
199. Valentim, B., Abagiu, A., Anghelescu, L., Flores, D., French, D., Goncalves, P., . . . Ward, C. (2019). Assessment of Bottom Ash Landfilled at Ceplea Valley (Romania) As A Source of Rare Earth Elements. International Journal of Coal Geology, 109-126.

200. Valkov, A., Sergievskiy, V., Sofronov, V., Kalaev, M., \& Ermakova, Y. (2014). Studies in the Field of Rare Earth Elements. Procedia Chemistry, 171-175.

201. Van Gosen , B., Vernplanck, P., \& Emsbo, P. (2019). Rare Earth Element Mineral Deposits in the United States. U.S.Geological Survey Circular 1454.

202. Van Gosen, B., Verplanck, P., Seal, R. I., Long, K., \& Gambogi, J. (2017). RareEarth Elements, Chap. O Schulz, K.J, DeYoung, J.H. Jr, Seal, R.R., II, and Bradley, D.C., eds. Critical Mineral Resources of United States-Economic and Environmental Geology and Prospects for Future Supply: U.S. Geological Survey Professional Paper. Retrieved from https://doi.org/10.3133/pp1802O

203. Vass, C. (2019). A Critical Assessment on the Resources and Extraction of Rare Earth Elements from Acid Mine Drainage . Morgantown, WV: Ph.D. Thesis, West Virginia University.

204. Vijayalakshmi, R., Mishra, S., Singh, H., \& Gupta, C. (2001). Processing of Xenotime Concentrate by Sulphuric Acid Digestion and Selective Thorium Precipitation for Separation of Rare Earths. Hydrometallurgy, 75-80.

205. Wagner, N., \& Matiane, A. (2018). Rare Earth Elements in Select Main Karoo Basin (South Africa) Coal and Coal Ash Samples. International Journal of Coal Geology, 82-92.

206. Wang, D., Zhao, Z., Yu, Y., Dai, J., Deng, M., Zhao, T., \& Liu, L. (2018). Exploration and Research Progress on Ion-Adsorption Type REE Deposit in South China. China Geology, 415-424.

207. Wang, L., Huang, X., Yu, Y., Zhao, L., Wang, C., Feng, Z., . . Long, Z. (2017). Towards Cleaner Production of Rare Earth Elements from Bastnaesite in China. Journal of Cleaner Production, 231-242.

208. Wang, Y., Zhang, Z., Liu, Y., Cao, Z., Liu, Y., \& Li, Q. (2012). Adsorption of U(VI) from Aqueous Solution by the Carboxyl-Mesoporous Carbon. Chemical Engineering Journal, 246-253.

209. World Nuclear Association. (2021, 06 04). Naturally-Occurring Radioactive Materials (NORM). Retrieved from World Nuclear Association: https://worldnuclear.org/information-library/safety-and-security/radiation-and-health/naturallyoccurring-radioactive-materials-norm.aspx

210. Xie, F., Zhang, T., Dreisinger, D., \& Doyle , F. (2014). A Critical Review on Solvent Extraction of Rare Earths from Aqueous Solutions. Minerals Engineering, 1028. 
211. Xiong, X., Tao, Y., Yu, Z., Yang, L., Sun, L., Fan, Y., \& Luo, F. (2020). Selective Extraction of Thorium from Uranium and Rare Earth Elements Using Sulfonated Covalent Organic Framework and Its Membrane Derivative. Chemical Engineering Journal, 123240.

212. Yadav, V., Gadi, R., \& Kalra, S. (2019). Clay Based Nanocomposites for Removal of Heavy Metals from Water: A Review. Journal of Environmental Maganement, 803-817.

213. Yakout, S. (2017). Influence of Solution Chemistry on the Selective Adsorption of Uranium and Thorium onto Activated Carbon. Nuclear Technology, 294-300.

214. Yang, X. (2019). Leaching Characteristics of Rare Earth Elements from Bituminous Coal-Based Sources. Lexington: Thesis, University of Kentucky.

215. Yoon, H., Kim, C., Chung, K., Kim, S., Lee, J., \& Kumar, J. (2016). Solvent Extraction, Separation and Recovery of Dysprosium (Dy) and Neodymium (Nd) from Aqueous Solutions: Waste Recycling Strategies for Permanent Magnet Processing. Hydrometallurgy, 27-43.

216. Yorukoglu, A., Obut, A., Girgin, I. (2003). Effect of Thiourea on Sulphuric Acid Leaching of Bastnaesite. Hydrometallurgy,195-202.

217. Zendelska, A., Golomeova, M., Blazev, K., Krstev, B., Golomeov, B., \& Krstev, A. (2015). Adsorption of Copper Ions from Aqueous Solutions on Natural Zeolite. Environmental Protection Engineering, 17-36.

218. Zhang, J., \& Zhao, B. (2016). Separation Hydrometallurgy of Rare Earth Elements. Saskatoon: Springer.

219. Zhang, J., Zhao, B., \& Schreiner, B. (2016). Separation Hydrometallurgy of Rare Earth Elements. Saskatoon: Springer.

220. Zhang, W., \& Honaker, R. (2019). Calcination Pretreatment on Acid Leaching Characteristics of Rare Earth Elements from Middlings and Coarse Refuse Material Associated with A Bituminous Coal Source. Fuel, 130-145.

221. Zhang, W., \& Honaker, R. (2018). Rare Earth Elements Recovery Using Stages Precipitation from A Leachate Generated from Coarse Coal Refuse. International Journal of Coal Geology, 189-199.

222. Zhang, W., Rezaee, M., Bhagavatula, A., Li, Y., Groppo, J., \& Honaker, R. (2015). A Review of the Occurrence and Promising Recovery Methods of Rare Earth Elements from Coal and Coal By-Products. International Journal of Coal Preparation and Utilization, 295-330.

223. Zhou, H., Wang, Y., Guo, X., Dong, Y., Su, X., \& Sun, X. (2018). The Recovery of Rare Earth by A Novel Extraction and Precipitation Strategy using Functional Ionic Liquids. Journal of Molecular Liquids, 414-420. 
224. Zhou, L., Shang, C., Liu, Z., Huang, G., \& Adesina, A. (2012). Selective Adsorption of Uranium(VI) from Aqueous Solutions Using the Ion-Imprinted Magnetic Chitosan Resins. Journal of Colloid and Interface Science, 165-172.

225. Zhu, Z., Pranolo, Y., \& Cheng, C. (2015). Separation of Uranium and Thorium from Rare Earths for Rare Earth Production - A Review. Minerals Engineering, 185196.

226. Zou, W., Zhao, L., \& Han, R. (2009). Removal of Uranium (VI) by Fixed Bed Ion-Exchange Column Using Natural Zeolite Coated with Manganese Oxide . Chinese Journal of Chemical Engineering, 585-593. 
APPENDIX

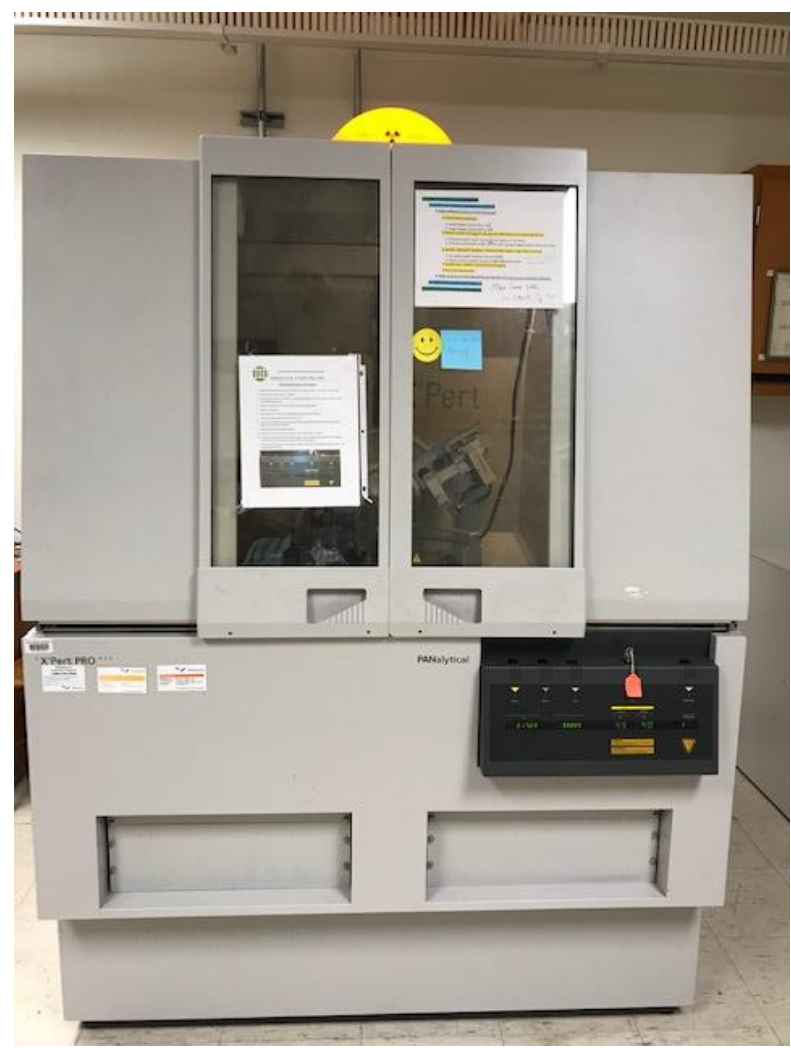

Figure 61 The PANanalytical XRD used throughout the study.

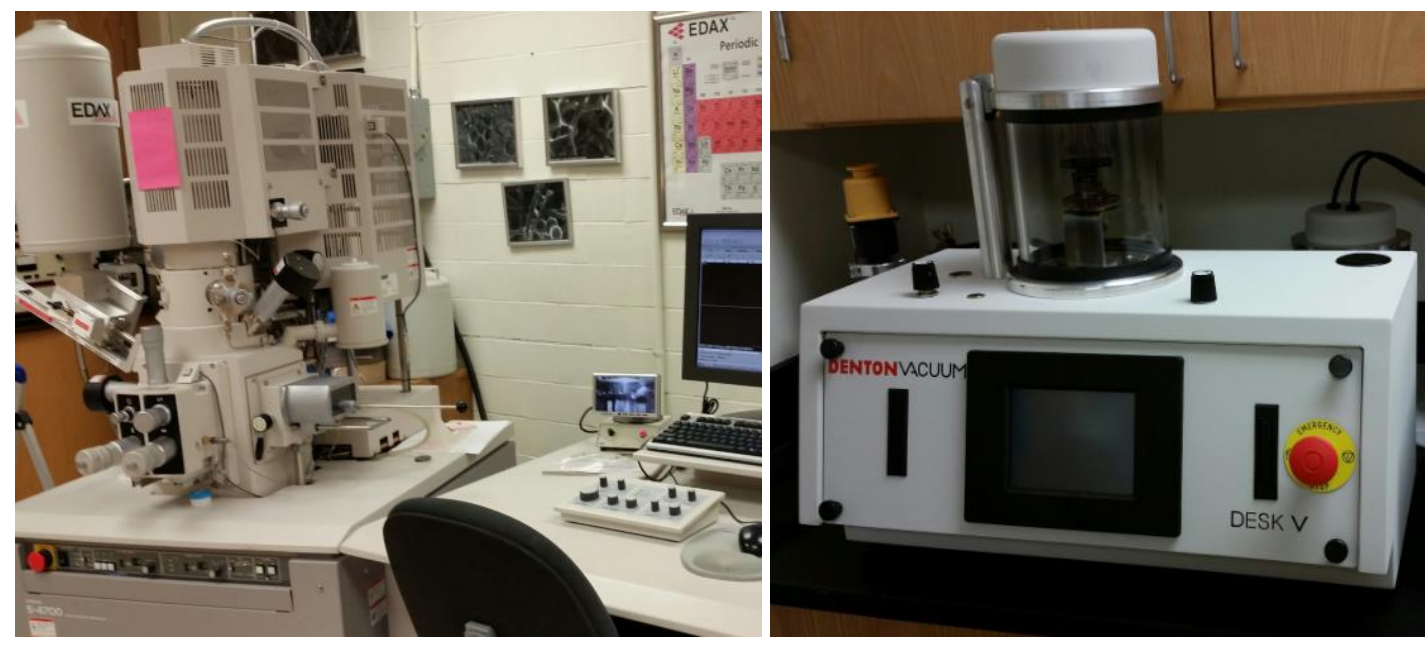

Figure 62 Instrument used for the SEM analysis of various solid samples. (A) Hitachi S4700 SEM-EDX (B) Denton Desk V Sputter and Carbon Coater. 


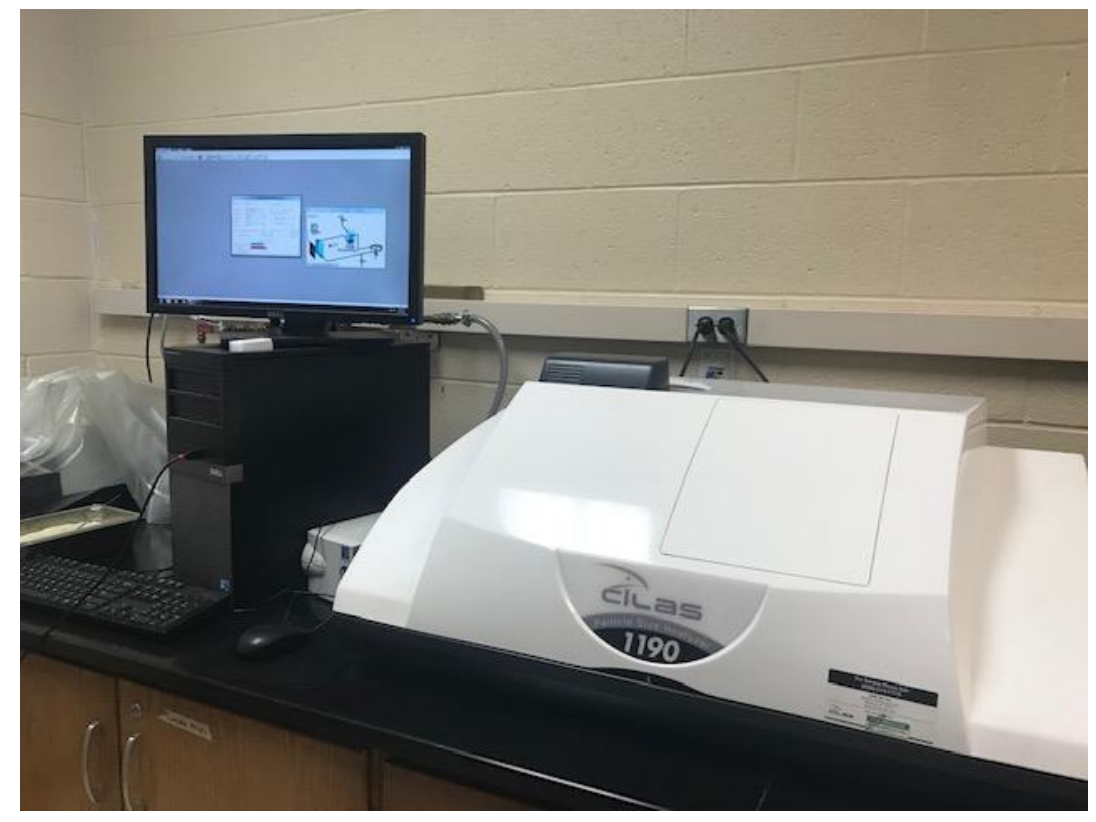

Figure 63 The CILAS particle size analyzer used throughout the study.

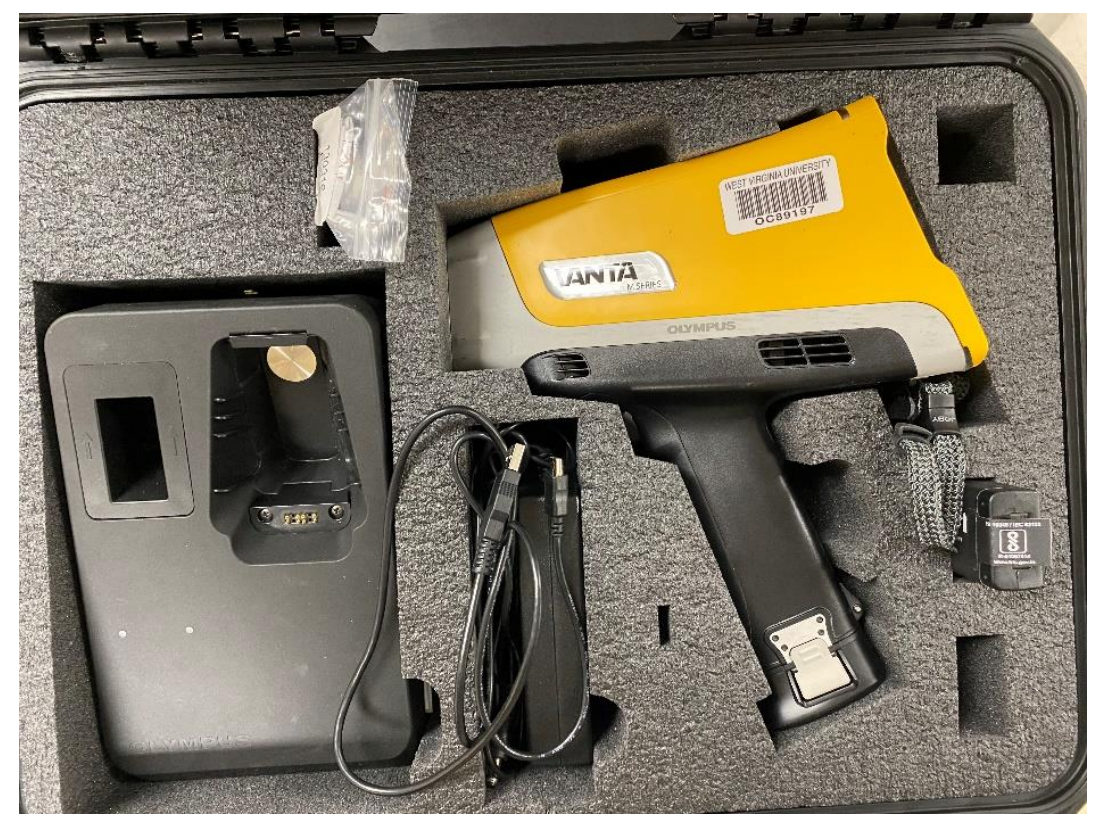

Figure 64 The Vanta handheld XRF used throughout the study. 


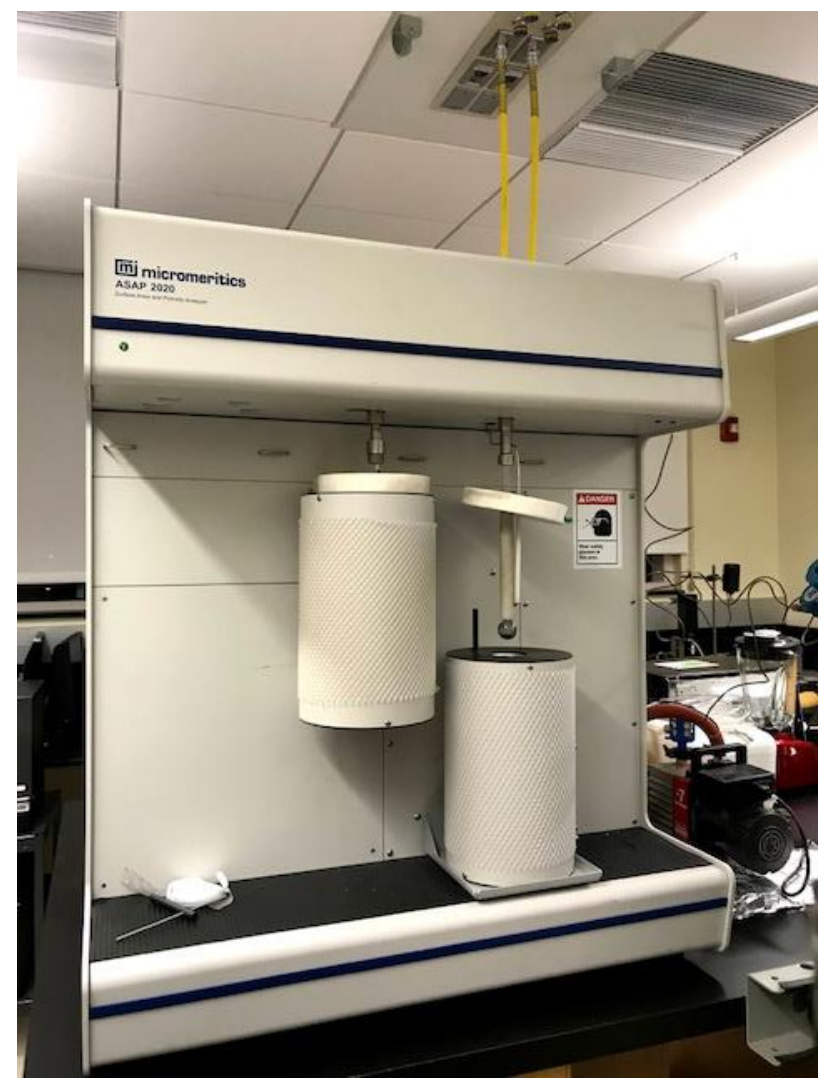

Figure 65 BET nitrogen adsorption instrument used for the characterization of zeolite samples.

Table 22 Individual rare earth elements, thorium and uranium concentrations (ppm) in the filtrates at various $\mathrm{pH}$ values.

\begin{tabular}{cccccccccc}
\hline Element/pH & 4.66 & 5.00 & 6.17 & 6.55 & 8.00 & 8.15 & 8.42 & 9.40 & 9.80 \\
\hline $\mathrm{Sc}$ & 0.00 & 0.00 & 0.00 & 0.00 & 0.00 & 0.00 & 0.00 & 0.00 & 0.00 \\
$\mathrm{Y}$ & 0.67 & 0.55 & 0.52 & 0.39 & 0.01 & 0.00 & 0.01 & 0.00 & 0.03 \\
$\mathrm{La}$ & 0.43 & 0.41 & 0.39 & 0.35 & 0.03 & 0.01 & 0.02 & 0.00 & 0.02 \\
$\mathrm{Ce}$ & 3.29 & 2.86 & 2.75 & 2.39 & 0.08 & 0.04 & 0.05 & 0.04 & 0.13 \\
$\mathrm{Pr}$ & 0.47 & 0.42 & 0.33 & 0.29 & 0.03 & 0.03 & 0.03 & 0.03 & 0.03 \\
$\mathrm{Nd}$ & 1.97 & 1.70 & 1.56 & 1.20 & 0.03 & 0.01 & 0.02 & 0.01 & 0.07 \\
$\mathrm{Sm}$ & 0.66 & 0.52 & 0.45 & 0.31 & 0.04 & 0.04 & 0.05 & 0.04 & 0.07 \\
$\mathrm{Eu}$ & 0.11 & 0.08 & 0.07 & 0.04 & 0.00 & 0.00 & 0.00 & 0.00 & 0.00 \\
$\mathrm{Gd}$ & 0.44 & 0.35 & 0.30 & 0.21 & 0.01 & 0.01 & 0.01 & 0.01 & 0.01 \\
$\mathrm{~Tb}$ & 0.02 & 0.02 & 0.02 & 0.02 & 0.02 & 0.02 & 0.02 & 0.02 & 0.02 \\
$\mathrm{Dy}$ & 0.18 & 0.14 & 0.10 & 0.06 & 0.01 & 0.01 & 0.01 & 0.01 & 0.01 \\
$\mathrm{Ho}$ & 0.02 & 0.02 & 0.02 & 0.01 & 0.00 & 0.00 & 0.00 & 0.00 & 0.00 \\
$\mathrm{Er}$ & 0.08 & 0.06 & 0.05 & 0.03 & 0.01 & 0.01 & 0.01 & 0.01 & 0.01 \\
$\mathrm{Tm}$ & 0.00 & 0.00 & 0.00 & 0.00 & 0.00 & 0.00 & 0.00 & 0.00 & 0.00 \\
$\mathrm{Yb}$ & 0.03 & 0.01 & 0.01 & 0.01 & 0.00 & 0.00 & 0.00 & 0.00 & 0.00 \\
$\mathrm{Lu}$ & 0.00 & 0.00 & 0.00 & 0.00 & 0.00 & 0.00 & 0.00 & 0.00 & 0.00 \\
$\mathrm{Th}$ & 0.01 & 0.00 & 0.00 & 0.00 & 0.00 & 0.00 & 0.00 & 0.00 & 0.00 \\
$\mathrm{U}$ & 0.15 & 0.04 & 0.00 & 0.00 & 0.00 & 0.00 & 0.00 & 0.00 & 0.01 \\
\hline
\end{tabular}


Table 23 Individual rare earth elements, thorium, and uranium precipitation (\%) at various $\mathrm{pH}$ values during the preliminary selective precipitation tests.

\begin{tabular}{cccccccccc}
\hline & 4.66 & 5.00 & 6.17 & 6.55 & 8.00 & 8.15 & 8.42 & 9.40 & 9.80 \\
\hline $\mathrm{Sc}$ & 79.09 & 80.20 & 79.09 & 100.00 & 100.00 & 100.00 & 100.00 & 100.00 & 100.00 \\
$\mathrm{Y}$ & 14.44 & 34.25 & 34.36 & 58.76 & 99.13 & 99.68 & 99.24 & 99.87 & 96.99 \\
$\mathrm{La}$ & 12.75 & 21.96 & 21.61 & 39.26 & 94.28 & 97.74 & 95.97 & 99.19 & 96.56 \\
$\mathrm{Ce}$ & 16.81 & 31.53 & 30.52 & 48.28 & 98.15 & 99.15 & 98.76 & 99.01 & 96.98 \\
$\mathrm{Pr}$ & 18.84 & 31.85 & 35.30 & 55.67 & 96.07 & 96.19 & 95.66 & 95.66 & 95.89 \\
$\mathrm{Nd}$ & 17.50 & 32.44 & 34.47 & 56.75 & 98.92 & 99.68 & 99.24 & 99.62 & 97.10 \\
$\mathrm{Sm}$ & 11.00 & 33.39 & 39.04 & 63.74 & 95.63 & 95.17 & 93.69 & 94.62 & 91.85 \\
$\mathrm{Eu}$ & 19.35 & 40.05 & 49.49 & 68.54 & 96.91 & 97.00 & 96.98 & 96.98 & 97.14 \\
$\mathrm{Gd}$ & 15.15 & 35.80 & 42.15 & 67.04 & 99.03 & 99.06 & 98.85 & 98.85 & 98.00 \\
$\mathrm{~Tb}$ & 15.21 & 19.71 & 15.21 & 42.50 & 40.72 & 42.50 & 15.21 & 15.15 & 19.71 \\
$\mathrm{Dy}$ & 18.07 & 40.95 & 53.11 & 74.28 & 97.83 & 97.89 & 97.72 & 97.72 & 97.41 \\
$\mathrm{Ho}$ & 18.38 & 38.82 & 45.58 & 66.66 & 88.89 & 88.89 & 89.79 & 89.78 & 90.33 \\
$\mathrm{Er}$ & 19.11 & 41.54 & 49.97 & 71.47 & 88.62 & 88.96 & 87.22 & 87.21 & 87.89 \\
$\mathrm{Tm}$ & 0.00 & 0.00 & 0.00 & 0.00 & 0.00 & 0.00 & 0.00 & 0.00 & 0.00 \\
$\mathrm{Yb}$ & 21.57 & 65.34 & 63.39 & 88.82 & 100.00 & 100.00 & 100.00 & 100.00 & 100.00 \\
$\mathrm{Lu}$ & 33.95 & 79.15 & 55.96 & 59.74 & 58.50 & 59.74 & 77.98 & 77.96 & 79.15 \\
$\mathrm{Th}$ & 95.49 & 99.83 & 99.99 & 99.99 & 99.99 & 99.99 & 99.99 & 99.99 & 99.99 \\
$\mathrm{U}$ & 28.67 & 82.18 & 98.45 & 99.59 & 99.40 & 99.90 & 99.82 & 99.37 & 96.52 \\
$\mathrm{TREEs}$ & 16.32 & 32.46 & 33.95 & 54.44 & 97.71 & 98.49 & 97.89 & 98.36 & 98.37 \\
\hline
\end{tabular}

Table 24 Precipitation (\%) of heavy, light, critical, and uncritical rare earth elements at various $\mathrm{pH}$ values during the preliminary selective precipitation tests.

\begin{tabular}{cccccccccc}
\hline Element/pH & 4.66 & 5.00 & 6.17 & 6.55 & 8.00 & 8.15 & 8.42 & 9.40 & 9.80 \\
\hline TREEs & 16.32 & 32.46 & 33.95 & 54.44 & 97.71 & 98.49 & 97.89 & 98.36 & 98.37 \\
HREEs & 15.93 & 36.96 & 41.32 & 64.67 & 97.45 & 97.76 & 97.18 & 97.45 & 96.05 \\
LREEs & 16.38 & 31.41 & 32.25 & 52.11 & 97.70 & 98.66 & 98.05 & 98.56 & 96.44 \\
CREEs & 17.18 & 33.35 & 35.96 & 58.12 & 98.14 & 98.72 & 98.23 & 98.57 & 96.59 \\
UCREEs & 15.72 & 31.83 & 32.53 & 51.87 & 97.41 & 98.33 & 97.65 & 98.21 & 96.21 \\
\hline
\end{tabular}

Table 25 Extraction recovery (\%) of total rare earths, thorium, and uranium during the exploratory solvent extraction tests.

\begin{tabular}{ccc}
\hline Element/Recovery (\%) & One-Stage & Double-Stage \\
\hline TREEs & 89.97 & 96.59 \\
Thorium & 92.76 & 93.80 \\
Uranium & 51.46 & 42.74 \\
\hline
\end{tabular}


Table 26 Extraction recovery (\%) individual rare earth elements during the exploratory solvent extraction tests.

\begin{tabular}{ccc}
\hline Element/Recovery (\%) & One-Stage & Double-Stage \\
\hline $\mathrm{Sc}$ & 63.76 & 62.54 \\
$\mathrm{Y}$ & 94.92 & 96.24 \\
$\mathrm{La}$ & 91.40 & 96.50 \\
$\mathrm{Ce}$ & 87.74 & 96.67 \\
$\mathrm{Pr}$ & 91.11 & 96.62 \\
$\mathrm{Nd}$ & 90.27 & 96.67 \\
$\mathrm{Sm}$ & 91.76 & 96.76 \\
$\mathrm{Eu}$ & 90.27 & 96.74 \\
$\mathrm{Gd}$ & 90.69 & 96.27 \\
$\mathrm{~Tb}$ & 94.15 & 95.42 \\
$\mathrm{Dy}$ & 89.83 & 96.64 \\
$\mathrm{Ho}$ & 91.79 & 96.39 \\
$\mathrm{Er}$ & 96.03 & 96.35 \\
$\mathrm{Tm}$ & 78.70 & 92.49 \\
$\mathrm{Yb}$ & 92.49 & 95.94 \\
$\mathrm{Lu}$ & 98.37 & 94.94 \\
$\mathrm{Th}$ & 92.76 & 93.80 \\
$\mathrm{U}$ & 51.46 & 42.74 \\
\hline
\end{tabular}

Table 27 Extraction recovery (\%) of heavy, light, critical, and uncritical rare earth elements during the exploratory solvent extraction tests.

\begin{tabular}{ccc}
\hline Element/Recovery (\%) & One-Stage & Double-Stage \\
\hline TREEs & 89.97 & 95.59 \\
HREEs & 92.79 & 96.31 \\
LREEs & 89.31 & 96.66 \\
CREEs & 91.32 & 96.57 \\
UCREEs & 89.01 & 96.66 \\
\hline
\end{tabular}

Table 28 Precipitation (\%) of total rare earth elements, thorium, uranium, and major metals during the one-stage modified experimental protocol.

\begin{tabular}{ccccc}
\hline Element/pH & TREEs & Thorium & Uranium & Major Metals \\
\hline 4.85 & 19.32 & 99.90 & 47.89 & 24.57 \\
\hline
\end{tabular}


Table 29 Individual rare earth elements and uranium extraction recovery (\%) into the final rare earth product stream based on solely solvent extraction circuit during the first subset of solvent extraction tests.

\begin{tabular}{|c|c|c|c|c|c|c|c|c|c|c|c|c|c|c|c|}
\hline Element/Recovery $(\%)$ & 1 & 2 & 3 & 4 & 5 & 6 & 7 & 8 & 9 & 10 & 11 & 12 & 13 & 14 & 15 \\
\hline $\mathrm{Sc}$ & 0.00 & 0.00 & 0.00 & 0.00 & 0.00 & 0.00 & 0.00 & 0.00 & 0.00 & 0.00 & 0.00 & 0.00 & 0.00 & 0.00 & 0.00 \\
\hline $\mathrm{Y}$ & 99.43 & 97.72 & 100.00 & 98.54 & 99.34 & 99.51 & 100.00 & 100.00 & 89.73 & 93.91 & 95.00 & 92.47 & 93.30 & 97.68 & 96.17 \\
\hline $\mathrm{La}$ & 96.76 & 98.75 & 99.41 & 97.09 & 98.89 & 96.78 & 94.12 & 98.39 & 90.24 & 95.50 & 95.97 & 93.17 & 94.90 & 97.76 & 97.59 \\
\hline $\mathrm{Ce}$ & 97.95 & 95.98 & 99.55 & 97.82 & 98.38 & 99.08 & 92.74 & 100.00 & 89.63 & 94.36 & 95.18 & 92.52 & 93.76 & 96.65 & 96.33 \\
\hline $\operatorname{Pr}$ & 94.88 & 88.89 & 98.49 & 94.09 & 98.62 & 95.03 & 100.00 & 97.98 & 91.80 & 87.25 & 87.69 & 87.12 & 87.12 & 93.22 & 82.67 \\
\hline $\mathrm{Nd}$ & 97.64 & 95.83 & 98.00 & 96.36 & 97.45 & 95.43 & 97.13 & 96.95 & 89.73 & 94.42 & 95.30 & 92.69 & 93.71 & 96.84 & 97.08 \\
\hline $\mathrm{Sm}$ & 97.39 & 94.98 & 97.76 & 95.82 & 96.94 & 94.85 & 94.03 & 97.04 & 90.94 & 93.74 & 93.99 & 93.59 & 93.58 & 95.05 & 91.00 \\
\hline $\mathrm{Eu}$ & 98.70 & 94.67 & 100.00 & 96.63 & 100.00 & 100.00 & 93.02 & 100.00 & 91.92 & 92.38 & 92.59 & 92.16 & 92.00 & 95.83 & 88.68 \\
\hline $\mathrm{Gd}$ & 98.23 & 94.60 & 98.74 & 97.62 & 98.83 & 97.40 & 93.79 & 99.18 & 91.65 & 94.39 & 96.52 & 93.11 & 92.60 & 96.79 & 97.09 \\
\hline $\mathrm{Tb}$ & 92.31 & 84.62 & 77.14 & 81.81 & 78.95 & 84.21 & 60.53 & 70.73 & 76.47 & 62.79 & 62.79 & 64.44 & 63.64 & 77.14 & 54.72 \\
\hline Dy & 100.00 & 96.75 & 100.00 & 100.00 & 100.00 & 100.00 & 96.40 & 100.00 & 90.96 & 94.68 & 97.06 & 93.37 & 93.33 & 98.73 & 99.38 \\
\hline Ho & 100.00 & 92.86 & 100.00 & 100.00 & 100.00 & 100.00 & 94.12 & 100.00 & 80.00 & 66.67 & 68.00 & 66.67 & 66.67 & 80.00 & 58.62 \\
\hline $\mathrm{Er}$ & 100.00 & 94.44 & 100.00 & 96.88 & 100.00 & 100.00 & 93.44 & 100.00 & 88.89 & 80.73 & 81.40 & 80.49 & 80.49 & 89.61 & 74.47 \\
\hline $\mathrm{Tm}$ & 0.00 & 0.00 & 0.00 & 0.00 & 0.00 & 0.00 & 0.00 & 0.00 & 0.00 & 0.00 & 0.00 & 0.00 & 0.00 & 0.00 & 0.00 \\
\hline $\mathrm{Yb}$ & 100.00 & 94.12 & 100.00 & 100.00 & 100.00 & 100.00 & 90.48 & 100.00 & 89.29 & 93.10 & 96.55 & 92.86 & 89.29 & 100.00 & 100.00 \\
\hline $\mathrm{Lu}$ & 0.00 & 0.00 & 0.00 & 0.00 & 0.00 & 0.00 & 0.00 & 0.00 & 0.00 & 0.00 & 0.00 & 0.00 & 0.00 & 0.00 & 0.00 \\
\hline $\mathrm{U}$ & 66.67 & 33.33 & 23.53 & 17.65 & 17.65 & 29.41 & 11.76 & 5.86 & 46.11 & 11.69 & 34.52 & 69.00 & 20.16 & 89.36 & 17.07 \\
\hline TREEs & 97.83 & 95.55 & 98.85 & 97.09 & 98.12 & 97.41 & 94.79 & 98.62 & 89.99 & 93.52 & 94.37 & 92.03 & 92.87 & 96.35 & 94.58 \\
\hline
\end{tabular}


Table 30 Individual rare earth elements and uranium concentrations $(\mathrm{mg} / \mathrm{L})$ in the final rare earth product stream based on solely solvent extraction circuit during the first subset of solvent extraction tests.

\begin{tabular}{|c|c|c|c|c|c|c|c|c|c|c|c|c|c|c|c|}
\hline Element/Concentration & 1 & 2 & 3 & 4 & 5 & 6 & 7 & 8 & 9 & 10 & 11 & 12 & 13 & 14 & 15 \\
\hline $\mathrm{Sc}$ & 0.00 & 0.47 & 0.00 & 0.00 & 0.00 & 0.00 & 0.00 & 0.00 & 0.00 & 0.00 & 0.00 & 0.00 & 0.00 & 0.00 & 0.00 \\
\hline Y & 0.52 & 0.32 & 0.55 & 0.54 & 0.61 & 0.61 & 0.53 & 0.62 & 0.60 & 0.65 & 0.65 & 0.62 & 0.63 & 0.63 & 0.65 \\
\hline $\mathrm{La}$ & 0.33 & 2.39 & 0.34 & 0.33 & 0.36 & 0.36 & 0.34 & 0.37 & 0.37 & 0.40 & 0.41 & 0.38 & 0.39 & 0.39 & 0.41 \\
\hline $\mathrm{Ce}$ & 2.53 & 0.22 & 2.67 & 2.60 & 2.91 & 2.92 & 2.39 & 2.97 & 2.79 & 3.06 & 3.04 & 2.88 & 2.96 & 2.94 & 3.04 \\
\hline $\operatorname{Pr}$ & 0.24 & 1.36 & 0.26 & 0.26 & 0.29 & 0.29 & 0.26 & 0.29 & 0.41 & 0.44 & 0.46 & 0.43 & 0.43 & 0.44 & 0.46 \\
\hline $\mathrm{Nd}$ & 1.45 & 0.59 & 1.57 & 1.53 & 1.68 & 1.69 & 1.52 & 1.72 & 1.73 & 1.88 & 1.89 & 1.79 & 1.82 & 1.81 & 1.86 \\
\hline $\mathrm{Sm}$ & 0.06 & 0.07 & 0.70 & 0.69 & 0.76 & 0.77 & 0.65 & 0.79 & 0.57 & 0.60 & 0.63 & 0.58 & 0.58 & 0.60 & 0.61 \\
\hline $\mathrm{Eu}$ & 0.08 & 0.26 & 0.09 & 0.09 & 0.10 & 0.10 & 0.08 & 0.10 & 0.09 & 0.10 & 0.10 & 0.10 & 0.09 & 0.09 & 0.09 \\
\hline $\mathrm{Gd}$ & 0.28 & 0.02 & 0.31 & 0.33 & 0.34 & 0.34 & 0.29 & 0.36 & 0.38 & 0.40 & 0.42 & 0.39 & 0.39 & 0.39 & 0.40 \\
\hline $\mathrm{Tb}$ & 0.02 & 0.12 & 0.03 & 0.03 & 0.03 & 0.03 & 0.02 & 0.03 & 0.03 & 0.03 & 0.03 & 0.03 & 0.03 & 0.03 & 0.03 \\
\hline Dy & 0.13 & 0.01 & 0.15 & 0.15 & 0.16 & 0.16 & 0.13 & 0.16 & 0.15 & 0.16 & 0.17 & 0.16 & 0.15 & 0.16 & 0.16 \\
\hline Ho & 0.01 & 0.05 & 0.02 & 0.02 & 0.02 & 0.02 & 0.02 & 0.02 & 0.01 & 0.02 & 0.02 & 0.02 & 0.02 & 0.02 & 0.02 \\
\hline $\mathrm{Er}$ & 0.06 & 0.00 & 0.06 & 0.06 & 0.07 & 0.07 & 0.06 & 0.07 & 0.06 & 0.07 & 0.07 & 0.07 & 0.07 & 0.07 & 0.07 \\
\hline $\mathrm{Tm}$ & 0.00 & 0.02 & 0.00 & 0.00 & 0.00 & 0.00 & 0.00 & 0.00 & 0.00 & 0.00 & 0.00 & 0.00 & 0.00 & 0.00 & 0.00 \\
\hline $\mathrm{Yb}$ & 0.02 & 0.00 & 0.02 & 0.02 & 0.03 & 0.03 & 0.02 & 0.03 & 0.03 & 0.03 & 0.03 & 0.03 & 0.03 & 0.03 & 0.03 \\
\hline $\mathrm{Lu}$ & 0.00 & 0.00 & 0.00 & 0.00 & 0.00 & 0.00 & 0.00 & 0.00 & 0.00 & 0.00 & 0.00 & 0.00 & 0.00 & 0.00 & 0.00 \\
\hline $\mathrm{U}$ & 0.02 & 0.01 & 0.04 & 0.03 & 0.03 & 0.05 & 0.02 & 0.01 & 0.05 & 0.01 & 0.03 & 0.07 & 0.02 & 0.04 & 0.01 \\
\hline TREEs & 6.31 & 5.88 & 6.77 & 6.64 & 7.34 & 7.39 & 6.29 & 7.52 & 7.23 & 7.83 & 7.90 & 7.46 & 7.58 & 7.58 & 7.83 \\
\hline
\end{tabular}


Table 31 Overall extraction recovery $(\%)$ of total rare earth elements and uranium during the first subset of solvent extraction tests.

\begin{tabular}{ccc}
\hline Test Number & Extraction Recovery $(\%)$ of TREEs & Extraction Recovery $(\%)$ of Uranium \\
\hline 1 & 78.93 & 34.68 \\
2 & 77.09 & 17.34 \\
3 & 79.75 & 12.24 \\
4 & 78.33 & 9.18 \\
5 & 79.16 & 9.18 \\
6 & 78.59 & 15.30 \\
7 & 76.48 & 6.12 \\
8 & 79.57 & 3.05 \\
9 & 72.60 & 23.93 \\
10 & 75.45 & 5.72 \\
11 & 76.14 & 17.69 \\
12 & 74.25 & 35.89 \\
13 & 74.93 & 10.40 \\
14 & 77.74 & 46.49 \\
15 & 76.31 & 8.88 \\
\hline
\end{tabular}


Table 32 Individual rare earths and uranium extraction recovery (\%) into the final rare earth product stream based solely on the solvent extraction circuit during the second subset of solvent extraction tests.

\begin{tabular}{ccccccccccccccccc}
\hline Element/Test & 1 & 2 & 3 & 4 & 5 & 6 & 7 & 8 & 9 & 10 & 11 & 12 & 13 & 14 & 15 \\
\hline $\mathrm{Sc}$ & 0.00 & 0.00 & 0.00 & 0.00 & 0.00 & 0.00 & 0.00 & 0.00 & 0.00 & 0.00 & 0.00 & 0.00 & 0.00 & 0.00 & 0.00 \\
$\mathrm{Y}$ & 98.79 & 96.72 & 97.55 & 98.15 & 98.48 & 92.87 & 93.17 & 94.23 & 95.84 & 96.30 & 95.32 & 98.100 & 70.211 & 95.389 & 95.76 \\
$\mathrm{La}$ & 98.53 & 97.8 & 97.52 & 97.92 & 98.04 & 96.15 & 95.89 & 94.21 & 95.53 & 95.94 & 95.24 & 98.04 & 75.99 & 96.47 & 98.99 \\
$\mathrm{Ce}$ & 97.82 & 96.08 & 96.00 & 97.19 & 95.87 & 90.94 & 90.11 & 92.31 & 93.60 & 93.50 & 93.20 & 95.57 & 76.37 & 96.10 & 96.53 \\
$\mathrm{Pr}$ & 96.65 & 92.56 & 87.86 & 96.85 & 88.17 & 87.33 & 85.00 & 84.89 & 84.61 & 86.56 & 87.55 & 78.97 & 84.11 & 95.87 & 95.08 \\
$\mathrm{Nd}$ & 97.98 & 96.00 & 96.69 & 97.28 & 96.14 & 91.32 & 91.32 & 92.44 & 93.90 & 94.19 & 93.41 & 95.91 & 78.28 & 94.81 & 96.28 \\
$\mathrm{Sm}$ & 93.24 & 86.35 & 92.40 & 92.72 & 91.63 & 81.97 & 80.71 & 91.99 & 90.58 & 89.01 & 91.93 & 90.12 & 86.82 & 95.55 & 97.18 \\
$\mathrm{Eu}$ & 98.06 & 90.09 & 96.15 & 98.17 & 92.79 & 87.76 & 90.14 & 90.98 & 91.30 & 91.11 & 91.75 & 91.67 & 73.63 & 95.18 & 96.08 \\
$\mathrm{Gd}$ & 98.14 & 93.36 & 96.77 & 97.660 & 95.96 & 88.65 & 91.23 & 92.36 & 93.38 & 93.72 & 93.22 & 95.48 & 72.80 & 96.30 & 95.38 \\
$\mathrm{~Tb}$ & 88.24 & 77.14 & 78.95 & 88.89 & 65.96 & 61.91 & 60.64 & 58.62 & 60.98 & 60.98 & 62.79 & 61.90 & 55.56 & 96.58 & 50.00 \\
$\mathrm{Dy}$ & 98.32 & 89.72 & 98.31 & 98.94 & 98.90 & 89.02 & 90.57 & 94.60 & 97.37 & 97.31 & 97.52 & 98.73 & 71.91 & 96.44 & 97.62 \\
$\mathrm{Ho}$ & 90.00 & 80.95 & 82.61 & 90.91 & 70.37 & 65.22 & 67.89 & 67.57 & 65.22 & 63.64 & 66.67 & 66.67 & 61.29 & 95.61 & 78.95 \\
$\mathrm{Er}$ & 94.94 & 89.47 & 90.48 & 95.24 & 82.80 & 80.00 & 80.64 & 78.75 & 79.75 & 79.49 & 80.73 & 80.73 & 72.15 & 96.30 & 89.75 \\
$\mathrm{Tm}$ & 0.00 & 0.00 & 0.00 & 0.00 & 0.00 & 0.00 & 0.00 & 0.00 & 0.00 & 0.00 & 0.00 & 0.00 & 0.00 & 0.00 & 20.00 \\
$\mathrm{Yb}$ & 100.00 & 82.15 & 100.00 & 100.00 & 100.00 & 83.33 & 100.00 & 100.00 & 100.00 & 100.00 & 100.000 & 100.000 & 56.53 & 98.84 & 86.21 \\
$\mathrm{Lu}$ & 0.00 & 20.00 & 100.00 & 100.00 & 100.00 & 100.00 & 100.00 & 100.00 & 0.00 & 0.00 & 100.00 & 100.00 & 41.09 & 95.95 & 20.00 \\
$\mathrm{U}$ & 62.47 & 4.28 & 42.92 & 59.39 & 57.22 & 12.00 & 29.95 & 3.42 & 23.33 & 31.68 & 10.00 & 22.58 & 5.14 & 30.00 & 5.46 \\
$\mathrm{TREEs}$ & 97.57 & 94.60 & 95.37 & 97.09 & 94.83 & 89.61 & 80.23 & 91.00 & 92.94 & 92.87 & 92.79 & 94.00 & 81.53 & 94.22 & 95.72 \\
\hline & & & & & & & & & & & & &
\end{tabular}


Table 32 continued

\begin{tabular}{|c|c|c|c|c|c|c|c|c|c|c|c|c|}
\hline Element/Test & 16 & 17 & 18 & 19 & 20 & 21 & 22 & 23 & 24 & 25 & 26 & 27 \\
\hline Sc & 20.00 & 20.00 & 14.29 & 33.33 & 20.00 & 23.53 & 20.00 & 20.00 & 20.00 & 33.33 & 14.28 & 14.28 \\
\hline Y & 96.15 & 96.81 & 97.22 & 95.92 & 97.35 & 95.94 & 95.87 & 96.05 & 94.18 & 93.25 & 96.13 & 96.20 \\
\hline $\mathrm{La}$ & 98.93 & 98.93 & 98.45 & 97.57 & 98.91 & 98.11 & 97.96 & 97.66 & 96.41 & 94.95 & 96.47 & 96.47 \\
\hline $\mathrm{Ce}$ & 95.40 & 95.88 & 95.23 & 95.41 & 96.28 & 94.85 & 94.97 & 95.29 & 93.05 & 93.15 & 95.29 & 95.00 \\
\hline $\operatorname{Pr}$ & 94.71 & 94.66 & 92.69 & 95.50 & 94.68 & 93.80 & 94.97 & 94.82 & 92.72 & 95.14 & 92.56 & 93.72 \\
\hline $\mathrm{Nd}$ & 95.17 & 95.63 & 95.44 & 95.29 & 96.05 & 94.70 & 95.08 & 95.22 & 93.38 & 92.63 & 94.45 & 93.92 \\
\hline $\mathrm{Sm}$ & 93.61 & 95.77 & 95.81 & 95.08 & 97.33 & 96.04 & 95.64 & 95.15 & 93.67 & 96.31 & 96.18 & 96.10 \\
\hline $\mathrm{Eu}$ & 95.87 & 95.83 & 94.18 & 96.81 & 95.83 & 96.70 & 95.96 & 95.79 & 93.75 & 94.57 & 93.94 & 96.55 \\
\hline $\mathrm{Gd}$ & 94.72 & 95.19 & 95.83 & 94.63 & 96.08 & 95.15 & 94.90 & 95.16 & 93.29 & 95.95 & 93.75 & 95.10 \\
\hline $\mathrm{Tb}$ & 47.83 & 47.83 & 39.40 & 63.64 & 46.67 & 51.85 & 48.94 & 47.83 & 48.94 & 63.64 & 39.98 & 48.99 \\
\hline Dy & 97.48 & 97.50 & 96.44 & 96.84 & 97.47 & 98.00 & 97.59 & 97.50 & 95.65 & 96.13 & 96.38 & 98.25 \\
\hline Ho & 78.95 & 78.95 & 71.43 & 87.50 & 77.78 & 82.20 & 78.95 & 77.78 & 77.78 & 86.67 & 71.43 & 80.77 \\
\hline $\mathrm{Er}$ & 89.33 & 89.19 & 85.19 & 94.21 & 89.19 & 91.28 & 89.33 & 88.89 & 88.89 & 93.85 & 84.62 & 92.18 \\
\hline $\mathrm{Tm}$ & 20.00 & 20.00 & 14.28 & 33.33 & 20.00 & 23.53 & 20.00 & 20.00 & 20.00 & 33.33 & 14.28 & 14.28 \\
\hline $\mathrm{Yb}$ & 86.21 & 86.21 & 81.25 & 92.31 & 85.72 & 88.50 & 85.19 & 84.62 & 84.00 & 91.30 & 78.57 & 91.69 \\
\hline $\mathrm{Lu}$ & 20.00 & 20.00 & 14.28 & 33.33 & 20.00 & 23.53 & 20.00 & 20.00 & 20.00 & 33.33 & 14.28 & 14.28 \\
\hline $\mathrm{U}$ & 22.58 & 11.87 & 12.73 & 36.77 & 43.33 & 40.43 & 13.73 & 60.78 & 21.57 & 50.98 & 7.27 & 37.26 \\
\hline TREEs & 94.65 & 95.20 & 94.43 & 95.16 & 95.64 & 94.63 & 94.61 & 94.70 & 92.80 & 93.41 & 93.92 & 94.93 \\
\hline
\end{tabular}


Table 33 Individual rare earth elements and uranium concentrations $(\mathrm{mg} / \mathrm{L})$ in the final rare earth product stream based solely on solvent extraction unit during the second subset of solvent extraction tests.

\begin{tabular}{cccccccccccccccc}
\hline Element/Concentration & 1 & 2 & 3 & 4 & 5 & 6 & 7 & 8 & 9 & 10 & 11 & 12 & 13 & 14 & 15 \\
\hline $\mathrm{Sc}$ & 0.00 & 0.00 & 0.00 & 0.00 & 0.00 & 0.00 & 0.00 & 0.00 & 0.00 & 0.00 & 0.00 & 0.00 & 0.00 & 0.00 & 0.00 \\
$\mathrm{Y}$ & 0.65 & 0.62 & 0.64 & 0.69 & 0.65 & 0.60 & 0.59 & 0.78 & 0.60 & 0.57 & 0.61 & 0.62 & 0.71 & 0.60 & 0.63 \\
$\mathrm{La}$ & 0.40 & 0.40 & 0.39 & 0.42 & 0.40 & 0.40 & 0.41 & 0.49 & 0.39 & 0.38 & 0.40 & 0.40 & 0.44 & 0.40 & 0.39 \\
$\mathrm{Ce}$ & 3.09 & 2.99 & 3.00 & 3.36 & 3.06 & 2.79 & 2.78 & 3.71 & 2.87 & 2.73 & 2.93 & 2.98 & 3.33 & 3.00 & 3.12 \\
$\mathrm{Pr}$ & 0.46 & 0.45 & 0.46 & 0.49 & 0.48 & 0.44 & 0.48 & 0.56 & 0.42 & 0.41 & 0.45 & 0.44 & 0.51 & 0.45 & 0.46 \\
$\mathrm{Nd}$ & 1.95 & 1.85 & 1.90 & 2.08 & 1.94 & 1.81 & 1.85 & 2.35 & 1.76 & 1.69 & 1.81 & 1.83 & 2.08 & 2.00 & 1.87 \\
$\mathrm{Sm}$ & 0.65 & 0.60 & 0.64 & 0.70 & 0.67 & 0.58 & 0.60 & 0.76 & 0.56 & 0.55 & 0.59 & 0.58 & 0.68 & 0.62 & 0.62 \\
$\mathrm{Eu}$ & 0.10 & 0.09 & 0.10 & 0.11 & 0.10 & 0.09 & 0.10 & 0.12 & 0.08 & 0.08 & 0.09 & 0.09 & 0.11 & 0.10 & 0.10 \\
$\mathrm{Gd}$ & 0.42 & 0.39 & 0.42 & 0.45 & 0.43 & 0.38 & 0.45 & 0.51 & 0.37 & 0.36 & 0.39 & 0.38 & 0.44 & 0.41 & 0.41 \\
$\mathrm{~Tb}$ & 0.03 & 0.03 & 0.03 & 0.03 & 0.03 & 0.03 & 0.03 & 0.03 & 0.03 & 0.03 & 0.03 & 0.03 & 0.03 & 0.02 & 0.02 \\
$\mathrm{Dy}$ & 0.18 & 0.16 & 0.18 & 0.19 & 0.18 & 0.15 & 0.20 & 0.21 & 0.15 & 0.15 & 0.16 & 0.16 & 0.18 & 0.16 & 0.16 \\
$\mathrm{Ho}$ & 0.02 & 0.02 & 0.02 & 0.02 & 0.02 & 0.02 & 0.02 & 0.03 & 0.02 & 0.01 & 0.02 & 0.02 & 0.02 & 0.02 & 0.02 \\
$\mathrm{Er}$ & 0.08 & 0.07 & 0.08 & 0.08 & 0.08 & 0.06 & 0.06 & 0.09 & 0.06 & 0.06 & 0.07 & 0.07 & 0.08 & 0.07 & 0.07 \\
$\mathrm{Tm}$ & 0.00 & 0.00 & 0.00 & 0.00 & 0.00 & 0.00 & 0.00 & 0.00 & 0.00 & 0.00 & 0.00 & 0.00 & 0.00 & 0.00 & 0.00 \\
$\mathrm{Yb}$ & 0.03 & 0.02 & 0.03 & 0.03 & 0.03 & 0.02 & 0.02 & 0.03 & 0.02 & 0.02 & 0.02 & 0.02 & 0.03 & 0.03 & 0.03 \\
$\mathrm{Lu}$ & 0.00 & 0.00 & 0.00 & 0.00 & 0.00 & 0.00 & 0.00 & 0.00 & 0.00 & 0.00 & 0.00 & 0.00 & 0.00 & 0.00 & 0.00 \\
$\mathrm{U}$ & 0.12 & 0.01 & 0.08 & 0.11 & 0.10 & 0.02 & 0.02 & 0.00 & 0.01 & 0.01 & 0.00 & 0.01 & 0.00 & 0.00 & 0.00 \\
$\mathrm{TREEs}$ & 8.15 & 7.69 & 7.89 & 8.64 & 8.06 & 7.35 & 7.61 & 9.66 & 7.30 & 7.04 & 7.57 & 7.61 & 8.63 & 7.88 & 7.91 \\
\hline
\end{tabular}


Table 33 continued

\begin{tabular}{ccccccccccccc}
\hline Element/Concentration & 16 & 17 & 18 & 19 & 20 & 21 & 22 & 23 & 24 & 25 & 26 & 27 \\
\hline $\mathrm{Sc}$ & 0.00 & 0.00 & 0.00 & 0.00 & 0.00 & 0.00 & 0.00 & 0.00 & 0.00 & 0.00 & 0.00 & 0.00 \\
$\mathrm{Y}$ & 0.60 & 0.61 & 0.63 & 0.59 & 0.59 & 0.63 & 0.60 & 0.58 & 0.58 & 0.55 & 0.60 & 0.60 \\
$\mathrm{La}$ & 0.37 & 0.37 & 0.38 & 0.36 & 0.36 & 0.38 & 0.38 & 0.38 & 0.38 & 0.36 & 0.38 & 0.38 \\
$\mathrm{Ce}$ & 2.90 & 2.93 & 3.06 & 2.85 & 2.85 & 3.03 & 3.02 & 2.91 & 2.92 & 2.76 & 2.97 & 3.00 \\
$\mathrm{Pr}$ & 0.43 & 0.43 & 0.46 & 0.42 & 0.43 & 0.45 & 0.45 & 0.44 & 0.43 & 0.41 & 0.45 & 0.45 \\
$\mathrm{Nd}$ & 1.74 & 1.75 & 1.83 & 1.70 & 1.70 & 1.81 & 1.82 & 1.75 & 1.75 & 1.65 & 1.79 & 1.80 \\
$\mathrm{Sm}$ & 0.59 & 0.59 & 0.62 & 0.58 & 0.58 & 0.61 & 0.61 & 0.59 & 0.59 & 0.57 & 0.61 & 0.61 \\
$\mathrm{Eu}$ & 0.09 & 0.09 & 0.10 & 0.09 & 0.09 & 0.10 & 0.10 & 0.09 & 0.09 & 0.09 & 0.09 & 0.09 \\
$\mathrm{Gd}$ & 0.39 & 0.40 & 0.41 & 0.39 & 0.39 & 0.40 & 0.41 & 0.39 & 0.39 & 0.38 & 0.41 & 0.40 \\
$\mathrm{~Tb}$ & 0.02 & 0.02 & 0.02 & 0.02 & 0.02 & 0.02 & 0.02 & 0.02 & 0.02 & 0.02 & 0.02 & 0.02 \\
$\mathrm{Dy}$ & 0.16 & 0.16 & 0.16 & 0.15 & 0.15 & 0.16 & 0.16 & 0.16 & 0.15 & 0.15 & 0.16 & 0.16 \\
$\mathrm{Ho}$ & 0.02 & 0.02 & 0.02 & 0.01 & 0.01 & 0.02 & 0.02 & 0.01 & 0.01 & 0.01 & 0.01 & 0.01 \\
$\mathrm{Er}$ & 0.07 & 0.07 & 0.07 & 0.07 & 0.07 & 0.07 & 0.07 & 0.06 & 0.06 & 0.06 & 0.07 & 0.07 \\
$\mathrm{Tm}$ & 0.00 & 0.00 & 0.00 & 0.00 & 0.00 & 0.00 & 0.00 & 0.00 & 0.00 & 0.00 & 0.00 & 0.00 \\
$\mathrm{Yb}$ & 0.03 & 0.03 & 0.03 & 0.02 & 0.02 & 0.03 & 0.02 & 0.02 & 0.02 & 0.02 & 0.02 & 0.02 \\
$\mathrm{Lu}$ & 0.00 & 0.00 & 0.00 & 0.00 & 0.00 & 0.00 & 0.00 & 0.00 & 0.00 & 0.00 & 0.00 & 0.00 \\
$\mathrm{U}$ & 0.02 & 0.01 & 0.01 & 0.03 & 0.03 & 0.03 & 0.00 & 0.03 & 0.01 & 0.03 & 0.00 & 0.02 \\
$\mathrm{TREEs}$ & 7.40 & 7.45 & 7.76 & 7.26 & 7.28 & 7.68 & 7.69 & 7.42 & 7.41 & 7.04 & 7.58 & 7.63 \\
\hline
\end{tabular}


Table 34 Overall extraction recovery $(\%)$ of total rare earth elements and uranium during the first subset of solvent extraction tests.

\begin{tabular}{ccc}
\hline Test Number & Extraction Recovery $(\%)$ of TREEs & Extraction Recovery $(\%)$ of Uranium \\
\hline 1 & 77.11 & 31.84 \\
2 & 74.76 & 2.18 \\
3 & 75.37 & 21.87 \\
4 & 76.71 & 30.25 \\
5 & 74.94 & 29.16 \\
6 & 70.82 & 6.12 \\
7 & 63.41 & 15.26 \\
8 & 71.92 & 1.74 \\
9 & 73.45 & 11.89 \\
10 & 73.40 & 16.14 \\
11 & 73.33 & 5.10 \\
12 & 74.29 & 11.51 \\
13 & 64.43 & 2.62 \\
14 & 74.46 & 15.29 \\
15 & 75.65 & 2.78 \\
16 & 74.80 & 11.51 \\
17 & 75.24 & 6.05 \\
18 & 74.63 & 6.49 \\
19 & 75.20 & 18.74 \\
20 & 75.58 & 22.02 \\
21 & 74.79 & 20.60 \\
22 & 74.77 & 6.77 \\
23 & 74.84 & 30.97 \\
24 & 73.34 & 11.00 \\
25 & 73.82 & 25.98 \\
26 & 74.22 & 3.71 \\
27 & 75.02 & 18.99 \\
\hline & &
\end{tabular}

Table 35 RE Oxide precipitation at various $\mathrm{pH}$ values.

\begin{tabular}{ccccccccc}
\hline Element/pH & 4.6 & 5.5 & 7.0 & 7.5 & 8 & 8.5 & 9.5 & 10.0 \\
\hline TREEs & 11.48 & 15.83 & 36.24 & 37.00 & 74.80 & 81.08 & 99.65 & 99.74 \\
Thorium & 95.01 & 100.00 & 100.00 & 100.00 & 100.00 & 100.00 & 100.00 & 100.00 \\
\hline
\end{tabular}


Table 36 Adsorption recovery of total rare earths, thorium, and uranium during zeolite adsorption tests.

\begin{tabular}{cccc}
\hline Test Number & TREEs & Thorium & Uranium \\
\hline 1 & 45.88 & 98.81 & 98.46 \\
2 & 76.88 & 99.86 & 91.29 \\
3 & 73.33 & 99.88 & 94.35 \\
4 & 67.33 & 98.28 & 96.75 \\
5 & 90.67 & 99.89 & 94.28 \\
6 & 77.71 & 99.13 & 98.46 \\
7 & 83.64 & 99.97 & 96.60 \\
8 & 91.76 & 99.20 & 93.17 \\
9 & 21.36 & 98.79 & 50.40 \\
10 & 73.20 & 99.94 & 97.59 \\
11 & 20.43 & 99.17 & 89.60 \\
12 & 92.00 & 99.90 & 94.06 \\
13 & 84.21 & 99.33 & 80.09 \\
14 & 53.64 & 99.61 & 91.02 \\
15 & 38.92 & 99.96 & 97.31 \\
16 & 0.03 & 99.33 & 0.00 \\
17 & 14.09 & 99.61 & 37.64 \\
18 & 73.75 & 99.96 & 98.65 \\
19 & 89.00 & 99.28 & 92.42 \\
20 & 94.00 & 99.86 & 93.11 \\
21 & 49.29 & 99.92 & 90.87 \\
22 & 96.00 & 86.71 & 77.38 \\
23 & 2.67 & 36.67 & 0.00 \\
24 & 89.58 & 99.80 & 87.32 \\
25 & 92.00 & 98.83 & 81.55 \\
26 & 10.19 & 82.43 & 0.00 \\
27 & 1.01 & 72.52 & 0.00 \\
\hline & & & \\
\hline
\end{tabular}


Table 37 Individual rare earth elements, thorium and uranium concentrations $(\mathrm{mg} / \mathrm{L})$ in the aqueous filtrate after zeolite adsorption tests.

\begin{tabular}{|c|c|c|c|c|c|c|c|c|c|c|c|c|c|c|c|}
\hline Element/Concentration & 1 & 2 & 3 & 4 & 5 & 6 & 7 & 8 & 9 & 10 & 11 & 12 & 13 & 14 & 15 \\
\hline Sc & 0.00 & 0.00 & 0.00 & 0.00 & 0.00 & 0.00 & 0.00 & 0.00 & 0.00 & 0.00 & 0.00 & 0.00 & 0.00 & 0.00 & 0.00 \\
\hline Y & 0.49 & 0.22 & 0.27 & 0.26 & 0.10 & 0.13 & 0.12 & 0.07 & 0.74 & 0.18 & 0.62 & 0.05 & 0.19 & 0.37 & 0.55 \\
\hline $\mathrm{La}$ & 0.39 & 0.22 & 0.24 & 0.27 & 0.11 & 0.23 & 0.16 & 0.11 & 0.45 & 0.24 & 0.41 & 0.08 & 0.17 & 0.34 & 0.37 \\
\hline $\mathrm{Ce}$ & 2.35 & 0.91 & 1.10 & 1.43 & 0.37 & 1.06 & 0.61 & 0.32 & 3.33 & 1.21 & 2.90 & 0.24 & 0.64 & 1.95 & 2.48 \\
\hline $\operatorname{Pr}$ & 0.33 & 0.10 & 0.13 & 0.19 & 0.06 & 0.12 & 0.08 & 0.06 & 0.49 & 0.12 & 0.41 & 0.06 & 0.09 & 0.27 & 0.36 \\
\hline $\mathrm{Nd}$ & 1.35 & 0.35 & 0.47 & 0.70 & 0.14 & 0.43 & 0.25 & 0.15 & 2.01 & 0.51 & 1.72 & 0.10 & 0.31 & 1.08 & 1.45 \\
\hline $\mathrm{Sm}$ & 0.35 & 0.08 & 0.13 & 0.18 & 0.07 & 0.11 & 0.08 & 0.05 & 0.65 & 0.12 & 0.55 & 0.05 & 0.10 & 0.28 & 0.45 \\
\hline $\mathrm{Eu}$ & 0.05 & 0.01 & 0.01 & 0.02 & 0.01 & 0.01 & 0.01 & 0.01 & 0.10 & 0.01 & 0.09 & 0.01 & 0.01 & 0.04 & 0.07 \\
\hline $\mathrm{Gd}$ & 0.25 & 0.06 & 0.09 & 0.12 & 0.03 & 0.06 & 0.04 & 0.02 & 0.44 & 0.08 & 0.37 & 0.02 & 0.05 & 0.20 & 0.31 \\
\hline $\mathrm{Tb}$ & 0.03 & 0.03 & 0.03 & 0.03 & 0.03 & 0.03 & 0.03 & 0.03 & 0.03 & 0.03 & 0.03 & 0.03 & 0.03 & 0.03 & 0.03 \\
\hline Dy & 0.09 & 0.02 & 0.03 & 0.04 & 0.01 & 0.01 & 0.01 & 0.01 & 0.18 & 0.02 & 0.15 & 0.01 & 0.02 & 0.06 & 0.13 \\
\hline Нo & 0.01 & 0.01 & 0.01 & 0.01 & 0.01 & 0.01 & 0.01 & 0.01 & 0.03 & 0.01 & 0.02 & 0.02 & 0.01 & 0.01 & 0.02 \\
\hline $\mathrm{Er}$ & 0.04 & 0.02 & 0.02 & 0.02 & 0.02 & 0.02 & 0.02 & 0.02 & 0.08 & 0.02 & 0.07 & 0.01 & 0.02 & 0.03 & 0.05 \\
\hline $\mathrm{Tm}$ & 0.00 & 0.00 & 0.00 & 0.00 & 0.01 & 0.01 & 0.01 & 0.00 & 0.00 & 0.00 & 0.00 & 0.00 & 0.00 & 0.00 & 0.00 \\
\hline $\mathrm{Yb}$ & 0.01 & 0.00 & 0.00 & 0.00 & 0.00 & 0.00 & 0.00 & 0.00 & 0.03 & 0.00 & 0.03 & 0.00 & 0.00 & 0.01 & 0.02 \\
\hline $\mathrm{Lu}$ & 0.00 & 0.00 & 0.00 & 0.00 & 0.00 & 0.00 & 0.00 & 0.00 & 0.00 & 0.00 & 0.00 & 0.00 & 0.00 & 0.00 & 0.00 \\
\hline Th & 0.00 & 0.00 & 0.00 & 0.00 & 0.00 & 0.00 & 0.00 & 0.00 & 0.00 & 0.00 & 0.00 & 0.00 & 0.00 & 0.00 & 0.00 \\
\hline $\mathrm{U}$ & 0.00 & 0.02 & 0.01 & 0.01 & 0.01 & 0.00 & 0.01 & 0.02 & 0.13 & 0.01 & 0.02 & 0.01 & 0.05 & 0.02 & 0.01 \\
\hline
\end{tabular}


Table 37 continued

\begin{tabular}{ccccccccccccc}
\hline Element/Concentration & 16 & 17 & 18 & 19 & 20 & 21 & 22 & 23 & 24 & 25 & 26 & 27 \\
\hline $\mathrm{Sc}$ & 0.00 & 0.00 & 0.00 & 0.00 & 0.00 & 0.00 & 0.00 & 0.00 & 0.00 & 0.00 & 0.00 & 0.00 \\
$\mathrm{Y}$ & 0.80 & 0.69 & 0.18 & 0.06 & 0.03 & 0.44 & 0.01 & 0.64 & 0.13 & 0.02 & 0.79 & 0.84 \\
$\mathrm{La}$ & 0.56 & 0.44 & 0.26 & 0.11 & 0.04 & 0.35 & 0.00 & 0.54 & 0.08 & 0.03 & 0.52 & 0.56 \\
$\mathrm{Ce}$ & 3.93 & 3.12 & 1.15 & 0.34 & 0.11 & 1.95 & 0.07 & 3.93 & 0.35 & 0.08 & 3.35 & 3.78 \\
$\mathrm{Pr}$ & 0.54 & 0.49 & 0.16 & 0.06 & 0.06 & 0.30 & 0.06 & 0.52 & 0.06 & 0.06 & 0.53 & 0.57 \\
$\mathrm{Nd}$ & 2.37 & 2.00 & 0.56 & 0.14 & 0.05 & 1.22 & 0.01 & 2.37 & 0.16 & 0.03 & 2.03 & 2.31 \\
$\mathrm{Sm}$ & 0.77 & 0.67 & 0.14 & 0.08 & 0.01 & 0.35 & 0.05 & 0.75 & 0.08 & 0.05 & 0.76 & 0.82 \\
$\mathrm{Eu}$ & 0.14 & 0.10 & 0.01 & 0.01 & 0.01 & 0.05 & 0.01 & 0.13 & 0.01 & 0.01 & 0.14 & 0.15 \\
$\mathrm{Gd}$ & 0.61 & 0.44 & 0.08 & 0.02 & 0.03 & 0.24 & 0.01 & 0.59 & 0.03 & 0.01 & 0.59 & 0.64 \\
$\mathrm{~Tb}$ & 0.03 & 0.03 & 0.03 & 0.03 & 0.01 & 0.03 & 0.03 & 0.03 & 0.03 & 0.03 & 0.03 & 0.03 \\
$\mathrm{Dy}$ & 0.25 & 0.18 & 0.02 & 0.01 & 0.01 & 0.08 & 0.01 & 0.25 & 0.01 & 0.01 & 0.25 & 0.27 \\
$\mathrm{Ho}$ & 0.03 & 0.03 & 0.01 & 0.01 & 0.02 & 0.01 & 0.01 & 0.03 & 0.01 & 0.01 & 0.03 & 0.04 \\
$\mathrm{Er}$ & 0.11 & 0.08 & 0.02 & 0.02 & 0.01 & 0.04 & 0.02 & 0.11 & 0.01 & 0.02 & 0.11 & 0.12 \\
$\mathrm{Tm}$ & 0.00 & 0.00 & 0.00 & 0.00 & 0.00 & 0.00 & 0.01 & 0.00 & 0.02 & 0.01 & 0.00 & 0.00 \\
$\mathrm{Yb}$ & 0.05 & 0.03 & 0.00 & 0.00 & 0.00 & 0.01 & 0.00 & 0.05 & 0.01 & 0.00 & 0.05 & 0.05 \\
$\mathrm{Lu}$ & 0.00 & 0.00 & 0.00 & 0.00 & 0.00 & 0.00 & 0.00 & 0.00 & 0.00 & 0.00 & 0.00 & 0.00 \\
$\mathrm{Th}$ & 0.00 & 0.00 & 0.00 & 0.00 & 0.00 & 0.00 & 0.02 & 0.08 & 0.00 & 0.00 & 0.02 & 0.04 \\
$\mathrm{U}$ & 0.30 & 0.14 & 0.00 & 0.02 & 0.01 & 0.02 & 0.05 & 0.30 & 0.03 & 0.04 & 0.20 & 0.30 \\
\hline
\end{tabular}

Table 38 The results of the kinetic adsorption tests.

\begin{tabular}{ccccccccc}
\hline Adsorption Recovery $(\%) /$ Time & 5 & 10 & 15 & 30 & 45 & 60 & 120 & 180 \\
\hline TREEs & 0.00 & 3.04 & 11.28 & 11.28 & 19.56 & 20.13 & 20.16 & 21.55 \\
Thorium & 86.47 & 86.76 & 87.48 & 87.48 & 89.70 & 90.60 & 91.02 & 93.41 \\
Uranium & 0.00 & 0.22 & 2.22 & 2.50 & 4.10 & 5.76 & 6.83 & 7.88 \\
\hline
\end{tabular}




\section{VITAE}

\section{EDUCATIONAL BACKGROUND}

2018- August 2021 Ph.D., Department of Mining Engineering, West Virginia University, Dissertation: Evaluation of Various Separation Techniques for the Removal of Actinides from A Rare Earth-containing Solution Generated from Coarse Coal Refuse.

Advisor: Qingqing Huang

2014-2016 MSc., Department of Mining Engineering, Middle East Technical University (METU), Turkey. Thesis: Beneficiation of Oxide Lead and Zinc Minerals by Selective Flotation and Ammonia Leaching.

Advisor: M. Ümit Atalay, Co-Advisor: N. Emre Altun

2008-2013 BSc, Department of Mining Engineering, Hacettepe University, Turkey,

02/2012-06/2012 Erasmus Exchange Student, University of Miskolc, Hungary

\section{RESEARCH \& WORK EXPERIENCE}

Graduate Research Assistant, West Virginia University Department of Mining Engineering 2018 - Present Researcher at a project funded by the US Department of Energy's National Energy Technology Laboratory

Project Name: Pilot-Scale Testing of an Integrated Circuit for the Extraction of Rare Earth Elements from Coal and Coal By-Products

02/2018 - 04/2018 Researcher at a project funded by WVU Office of Global Affairs, Energy Institute \& Office of Research

Project Name: Study of Rare Earth Elements from Colombian Coals for High-Tech Industries and Clean Energy Technologies

Research Assistant, Middle East Technical University Department of Mining Engineering, Ankara, Turkey

05/2017-12/2017 Researcher at a project funded by The Scientific and Technological Research Council of Turkey (TUBITAK)

Project Name: Obtaining Hybrid Fossil Fuel-Biomass Fuel Briquettes Based On Domestic Energy Resources (Project Code: 315M310)

2015-2016 Researcher at a project funded by General Directorate of Eti Mine Enterprises

Project Name: Preliminary Evaluation of Mineral Fields of General Directorate of Eti Mine Enterprises (Project Code: 2015-03-05-00-04) 
Researcher at a project funded by Middle East Technical University Coordination of Scientific Research Projects

Project Name: Production of Lead and Zinc Concentrates from Oxide Lead and Zinc Ores by Selective Flotation (Project Code: BAP-03-052015-001)

Summer 2013 Intern at Koza Gold Corporation, Ovacik Gold Mine, Izmir, Turkey

Summer 2012 Intern at Eldorado Gold/Tuprag Metal Madencilik San.\&Tic. A.S, Efemcukuru Gold Mine, Izmir, Turkey

\section{TEACHING EXPERIENCE}

Fall 2020, Mineral Processing, West Virginia University

Fall 2019, Aggregates Production, West Virginia University

Spring 2018, Coal Preparation, West Virginia University

2013-2017, Mineral Processing Laboratory I and II, Middle East Technical University

\section{JOURNAL PUBLICATIONS}

1. Talan, D., Huang, Q., (2021). Rare Earth Elements: A Review of Recent Advances in Resource Exploration, Environmental Aspect of Extraction and Solution Purification Techniques. Submitted to Minerals Engineering.

2. Talan, D., Huang, Q., (2021). Separation of Radionuclides from A Rare Earth-Containing Solution by Zeolite Adsorption. Minerals 11 (1). doi.org/10.3390/min11010020.

3. Huang, Q., Noble, A., Talan, D., (2020). Exploratory Investigation on the Use of Low-Cost Alternative Media for Ultrafine Grinding of Coal. International Journal of Coal Preparation and Utilization. DOI: 10.1080/19392699.2020.1809387

4. Talan, D., Huang, Q., (2020). Separation of Thorium, Uranium and Rare Earths from A Strip Solution Generated from Coarse Coal Refuse. Hydrometallurgy. Volume 197, 105446, DOI:10.1016/j.hydromet.2020.105446.

5. Huang, Q., Talan, D., Restrepo, J., Restrepo, O., Kecojevic, V., Noble, A. (2019). Characterization Study of Rare Earths, Yttrium and Scandium from Various Colombian Coal Samples, International Journal of Coal Geology. Volume 209, p 14-26, DOI:10.1016/j.coal.2019.04.008.

\section{CONFERENCE PUBLICATIONS}

1. Talan, D., Atalay, Ü. and Altun, N. E. (2017). Extraction of Zinc from Smithsonite by Ammonia Leaching. 4th International Conference on Mining, Material and Metallurgical Engineering (MMME'17), $8^{\text {th }}-10^{\text {th }}$ of June 2017, Rome-Italy.

2. Talan, D., Atalay, Ü. and Altun, N. E. (2015). Characterization of Kayseri-Develi Oxide Lead and Zinc Ore. XVI Balkan Mineral Processing Congress, $17^{\text {th }}-19^{\text {th }}$ of June 2015, Belgrade-Serbia. 


\section{TECHNICAL \& POSTER PRESENTATIONS}

1. Talan, D. and Huang, Q. (2021). Process Development for Separating Actinides from Rare Earth-containing Solution Generated from Coarse Coal Refuse. Poster presentation. 2021 SME Annual Conference \& Expo, Mineral and Metallurgical Division Student Poster Competition, Virtual Event.

2. Talan, D. and Huang, Q. (2021). Process Development for Separating Actinides from Rare Earth-containing Solution Generated from Coarse Coal Refuse. Poster presentation. 2021 SME Annual Conference \& Expo, Environmental Division Student Poster Competition, Virtual Event.

3. Talan, D. and Huang, Q. (2021). Process Development for Separating Actinides from Rare Earth-containing Solution Generated from Coarse Coal Refuse. Technical presentation. 2021 SME Annual Conference \& Expo, $1^{\text {st }}-5^{\text {th }}$ of March, Virtual Event.

4. Talan, D., Huang, Q., Honaker, R. (2020). Separation of Thorium and Uranium from Rare Earth Elements Using Zeolite Adsorption. Technical presentation. 2020 SME Annual Conference \& Expo, $23^{\text {rd }}-26^{\text {th }}$ of February, Phoenix-Arizona.

5. Talan, D., Huang, Q., (2020). Separation of Radionuclides from Rare Earth Elements using Zeolite Adsorption. Poster presentation. 2020 SME Annual Conference \& Expo, Graduate Student Research Poster Competition, $23^{\text {rd }}-26^{\text {th }}$ of February, Phoenix-Arizona.

6. Talan, D., Huang, Q., (2020) A Study on Removal of Thorium and Uranium from Rare Earth Elements using Zeolite Adsorption. Poster presentation. 2020 SME Annual Conference \& Expo Mineral and Metallurgical Division Student Poster Competition, 23 ${ }^{\text {rd }}$ $26^{\text {th }}$ of February, Phoenix-Arizona. (Ranked $6^{\text {th }}$ in 26 posters).

7. Talan, D., Huang, Q., Honaker, R., (2019). Removal of Radioactive Elements from Rare Earths Using Various Separation Methods. Technical presentation. 2019 SME Annual Conference \& Expo, $24^{\text {th }}-27^{\text {th }}$ of February, Denver-Colorado.

\section{SCHOLARSHIPS \& AWARDS}

1. Ranked $1^{\text {st }}$ at the 2021 Society of Mining, Metallurgy and Exploration (SME), Environmental Division Poster Contest

2. Ranked $5^{\text {th }}$ at the 2021 Society of Mining, Metallurgy and Exploration (SME), Mineral and Metallurgical Processing Division (MPD) Student Poster Competition

3. Finalist for 2021 Society of Mining Professors Michael Karmis Ph.D. Fellowship Award

4. Recipient of 2021 Mining and Metallurgical Society of America / Society of Mining, Metallurgy and Exploration Foundation Presidential Scholarship

5. Recipient of 2020 Outstanding Merit Fellowship for Continuing Doctoral Students (sole recipient among Benjamin M. Statler College of Engineering and Mineral Resources)

6. Recipient of 2020 Women's Auxiliary to the American Institute of Mining Engineers (WAAIME) Scholarship 
7. Ranked $6^{\text {th }}$ at the 2020 Society of Mining, Metallurgy and Exploration (SME), Mineral and Metallurgical Processing Division (MPD) Student Poster Competition

8. Recipient of 2019 WVU Mining Engineering Faculty Graduate Award

9. Recipient of 2019 Women's Auxiliary to the American Institute of Mining Engineers (WAAIME) Scholarship

10. Recipient of 2015 Performance Award (given by Middle East Technical University, and sole recipient) 